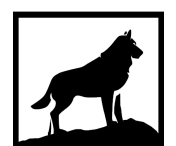

Michigan Technological

1 8 8 5 University
Michigan Technological University Digital Commons @ Michigan Tech

\title{
A COMPUTATIONAL STUDY OF PROPERTIES OF CORE-SHELL NANOWIRE HETEROSTRUCTURES USING DENSITY FUNCTIONAL THEORY
}

Sandip Aryal

Michigan Technological University

Copyright 2021 Sandip Aryal

Recommended Citation

Aryal, Sandip, "A COMPUTATIONAL STUDY OF PROPERTIES OF CORE-SHELL NANOWIRE HETEROSTRUCTURES USING DENSITY FUNCTIONAL THEORY", Open Access Dissertation, Michigan Technological University, 2021.

https://doi.org/10.37099/mtu.dc.etdr/1341

Follow this and additional works at: https://digitalcommons.mtu.edu/etdr

Part of the Condensed Matter Physics Commons 
A COMPUTATIONAL STUDY OF PROPERTIES OF CORE-SHELL NANOWIRE HETEROSTRUCTURES USING DENSITY FUNCTIONAL THEORY

\author{
By \\ Sandip Aryal
}

\begin{abstract}
A DISSERTATION
Submitted in partial fulfillment of the requirements for the degree of DOCTOR OF PHILOSOPHY
\end{abstract}

In Physics

MICHIGAN TECHNOLOGICAL UNIVERSITY

2021

C) 2021 Sandip Aryal 

This dissertation has been approved in partial fulfillment of the requirements for the Degree of DOCTOR OF PHILOSOPHY in Physics.

Department of Physics

Dissertation Advisor: Dr. Ranjit Pati

Committee Member: Dr. Ravindra Pandey

Committee Member: Dr. Maximilian J. Seel

Committee Member: Dr. Susanta Ghosh

Department Chair: Dr. Ravindra Pandey 



\section{Dedication}

To my family ... 



\section{Contents}

List of Figures $\ldots \ldots \ldots \ldots \ldots \ldots \ldots \ldots \ldots$

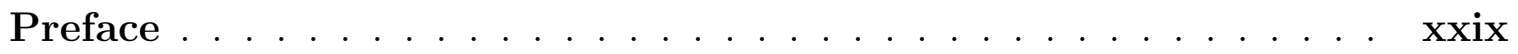

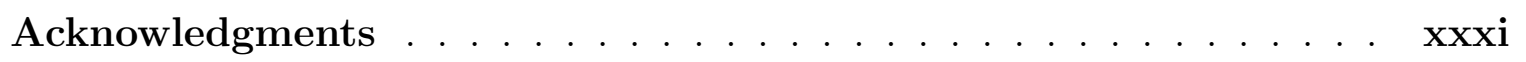

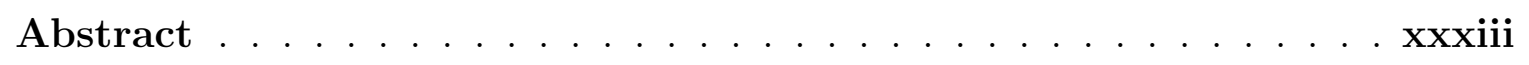

1 Introduction ........................... 1

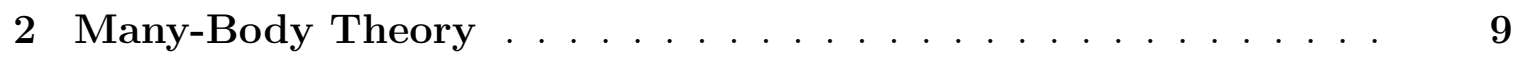

2.1 Many-Body Schrödinger Wave Equation $\ldots \ldots \ldots$

2.2 Born-Oppenheimer Approximation $\ldots \ldots \ldots \ldots \ldots$

2.3 Hellmann-Feynman Theorem . . . . . . . . . . . . . . . 14

2.4 Hartree-Fock Theory . . . . . . . . . . . . . . . . . . . . 15

2.5 Correlation Energy . . . . . . . . . . . . . . . . . . . . . . . . . 21

2.6 Density Functional Theory $(\mathrm{DFT}) \ldots \ldots \ldots \ldots \ldots$

2.6 .1 Why DFT? . . . . . . . . . . . . . 23

2.6.2 Thomas-Fermi Model . . . . . . . . . . . . . . . 2 25 
2.6.3 The Hohenberg-Kohn Theorem I . . . . . . . . . . 27

2.6.4 The Hohenberg-Kohn Theorem II . . . . . . . . . . . . . . 30

2.6.5 Levy Constrained Search .............. 31

2.6.6 The Kohn-Sham Formulation ............. 33

2.6.7 Exchange-Correlation Energy ............. 38

2.6.7.1 Local Density Approximation ......... 39

2.6.7.2 Generalized Gradient Approximation ....... 41

2.6.7.3 Hybrid Functional ............. 42

2.7 Bloch's Theorem and Plane-Wave Expansion . . . . . . . . . 44

2.8 k-point Sampling . . . . . . . . . . . . . . . . 47

2.9 Truncating Plane-Wave Basis Sets . . . . . . . . . 51

2.10 Pseudopotential Approximation ............. 53

2.11 Plane-Wave Representation of Kohn-Sham Equations . . . . . . . 56

2.12 Collinear and Noncollinear Magnetism . . . . . . . . . . 56

2.13 Projector Augmented Wave Method . . . . . . . . . . . . 62

2.14 Spin-Orbit Coupling in PAW Method . . . . . . . . . 63

3 Quantum Transport in a Nanoscale Junction . . . . . . . . . . 67

3.1 Fundamental Length Scales ............... 68

3.2 Modeling of a Nanoscale Device ............. 71

3.3 Landauer Formalism to Study Electron Transport . . . . . . . . . 73

3.4 Single Particle Green's Function Formalism . . . . . . . . . . . . . . 80 
3.5 Spin Transport in a Nanoscale Junction . . . . . . . . . . . . . 86

4 Mn-doped Ge/Si core/shell nanowire . . . . . . . . . . . . 89

4.1 Introduction . . . . . . . . . . . . . . . . . 89

4.2 Computational Method . . . . . . . . . . . . . 92

4.3 Results and Discussions . . . . . . . . . . . . . . 95

4.4 Conclusions ...................... 111

5 Cr-doped Ge/Si core/shell nanowire . . . . . . . . . . 113

5.1 Introduction . . . . . . . . . . . . . . . . 113

5.2 Computational Details ............... 116

5.3 Results and Discussions . . . . . . . . . . . . . . . . 119

5.4 Conclusions ........................ 136

$6 \mathrm{PbTe} / \mathrm{PbS}$ core/shell nanowire . . . . . . . . . . . . 137

6.1 Introduction . . . . . . . . . . . . . . . . 137

6.2 Computational Details . . . . . . . . . . . . . . . . 140

6.3 Results and discussion . . . . . . . . . . . . . . . 143

6.4 Conclusion ....................... 165

7 Conclusion and Future Perspectives . . . . . . . . . . 167

7.1 Conclusion . . . . . . . . . . . . . 167

7.2 Future Perspectives . . . . . . . . . . . . . . . 170 
References .......................... 173

A Copyrights ........................ 193

B Copyrights ....................... 197

C Copyrights ........................... 199 


\section{List of Figures}

2.1 An example of potential energy curve for the ground state (GS) obtained by solving Equation 2.5. $R_{e}$ is the equilibrium configuration of ions. . . . . . . . . . . . . . . . .

2.2 One to one mapping of the real interacting system with a noninteracting system. Both systems have the same ground state density

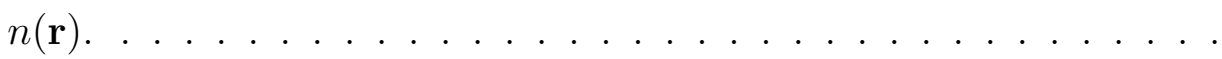

2.3 Planes waves can be represented by grids in reciprocal space; all plane waves within the cutoff energy are chosen for plane-wave expansion.

2.4 Schematic showing (a) all electron wave functions of the core and valence electrons (b) pseudo wave function and pseudo potential below and above the cut off radius $r_{c}$ and the corresponding all electron wave

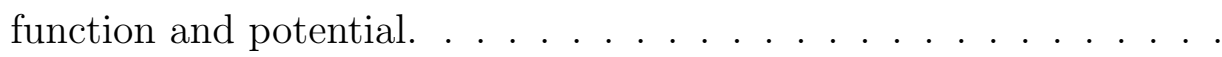

3.1 A schematic showing a two terminal nanowire junction; the nanowire together with the leads form an active scattering region. . . . . . 
4.1 The top view of the optimized unit cell structures of Mn-doped Gecore/Si-shell nanowire along the $\langle 110\rangle$ direction for different doping sites (I, II, III, and IV). The core diameter of the nanowire is $11.7 \AA$; the unsaturated surface states are passivated by $\mathrm{H}$-atoms. (Reproduced from Nanoscale Adv. 2020, 2, 1843-1849 with permission from Royal Society of Chemistry. $\ldots \ldots \ldots \ldots \ldots \ldots$

4.2 (a) Energy vs. Mn position in the nanowire; I, II, III, IV refer to the Mn positions.(b) Energy vs. Lattice parameter of the nanowire. The minimum energy is set to zero value in the energy scale for both (a) and (b). (Reproduced from Nanoscale Adv. 2020, 2, 1843-1849 with permission from Royal Society of Chemistry.) . . . . . . . . 96

4.3 Atom decomposed electronic band structure (PBE) of Mn-doped Ge-core/Si-shell nanowire in the (a-c) Minority-spin direction (d-f) Majority-spin direction; the circle represents the contribution of Ge, $\mathrm{Si}$, and $\mathrm{Mn}$ atoms to energy bands. (Reproduced from Nanoscale Adv. 2020, 2, 1843-1849 with permission from Royal Society of Chemistry.) 
4.4 (a) Schematic showing the local alignment of magnetization of Mn and nearest Ge atoms in the nanowire. (b) Calculated energy for paramagnetic (PM), ferromagnetic (FM), and antiferromagnetic (AFM) configurations; the energy of the most stable FM state is set to zero. (Reproduced from Nanoscale Adv. 2020, 2, 1843-1849 with permission from Royal Society of Chemistry.) . . . . . . . . . . . . .

4.5 Electronic band structure (HSE06) of Mn-doped Ge-core/Si-shell nanowire: (a) Minority-spin direction (b) Majority-spin direction. (Reproduced from Nanoscale Adv. 2020, 2, 1843-1849 with permission from Royal Society of Chemistry.) . . . . . . . . . . . . . . .

4.6 Atom and orbital decomposed density of states (DOS) of the Mndoped Ge-core/Si-shell nanowire in the (a) Minority-spin direction (b) Majority-spin direction. (Reproduced from Nanoscale Adv. 2020, 2, 1843-1849 with permission from Royal Society of Chemistry.) . . . . 102

4.7 (a) The electronic band structure (PBE) of the Mn-doped Ge-core/Sishell nanowire with and without spin-orbit (SO) coupling; MJ and MI refer to the majority and minority spin directions. (b) Magnified version of (a) depicting SO splitting at the crossing points of MJ and MI bands. (Reproduced from Nanoscale Adv. 2020, 2, 1843-1849 with permission from Royal Society of Chemistry.) . . . . . . . . . . 103 
4.8 Electronic band structure (PBE) of Mn-doped Ge-core/Si-shell nanowire in the (a) Minority-spin (b) Majority-spin direction when Mn atom is doped at site II. (c) and (d) represent the band structure in the Minority-spin and Majority-spin direction when Mn atom is doped at site III. (Reproduced from Nanoscale Adv. 2020, 2, 1843-1849 with permission from Royal Society of Chemistry.) . . . . . . . . . 104

4.9 (a) The top view of the optimized Mn-doped Ge-core/Si-shell nanowire along the $\langle 110\rangle$ direction (Mn at the interstitial site). The electronic band structure (PBE) of Mn-doped Ge-core/Si-shell nanowire in the (b) Minority-spin direction (c) Majority-spin direction when Mn is doped at the interstitial site; the half-metallic feature is noticeable. (Reproduced from Nanoscale Adv. 2020, 2, 1843-1849 with permission from Royal Society of Chemistry.) . . . . . . . . . . . . . 105 
4.10 The optimized unit cell structure of Mn-doped Ge-core/Si-shell nanowire when (a) both Mn atoms are doped in the core (b) one $\mathrm{Mn}$ atom is doped in the core and other in the shell. The electronic band structure in the (c) Minority-spin (d) Majority-spin direction when both Mn atoms are doped in core; (e) and (f) represent the energy band diagram in the Minority-spin and Majority-spin direction when one $\mathrm{Mn}$ atom is doped in core and other in the shell. (Reproduced from Nanoscale Adv. 2020, 2, 1843-1849 with permission from Royal Society of Chemistry.) . . . . . . . . . . . . . .

4.11 Electronic band structure (PBE) of Mn-doped Ge-core/Si-shell nanowire (Mn at the site I) under tensile strain along the nanowire axis. (a) and (b) represent the band structure in the Minority-spin and Majority-spin direction under lateral strain of $+1.26 \%$; (c) and (d) represent the band structures in the Minority-spin and Majority-spin direction under lateral strain of $+2.52 \%$. (Reproduced from Nanoscale Adv. 2020, 2, 1843-1849 with permission from Royal Society of Chemistry.) . . . . . . . . . . . . . . . . 107 
4.12 Electronic band structure (PBE) of Mn-doped Ge-core/Si-shell nanowire (Mn at site I) under compressive strain along the nanowire axis. (a) and (b) illustrate the band structures in the Minority-spin and Majority-spin direction under compressive strain of -1.26\%; (c) and (d) represent the band structures in the Minority-spin and Majorityspin direction under compressive strain of $-2.52 \%$. (Reproduced from Nanoscale Adv. 2020, 2, 1843-1849 with permission from Royal Society

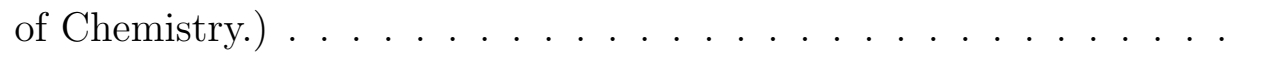

4.13 (a) A prototypical Mn-doped Ge-core/Si-shell nanowire spin-filter; the channel length is $\sim 2.37 \mathrm{~nm}$ (electrode-electrode distance). (b) Calculated spin-dependent transmission; $\mathrm{T}_{\mathrm{MJ}}$ and $\mathrm{T}_{\mathrm{MI}}$ refer to the transmission for the majority and minority spin carriers respectively. (Reproduced from Nanoscale Adv. 2020, 2, 1843-1849 with permission from Royal Society of Chemistry. . . . . . . . . . . . . . . . . 110

5.1 The optimized unit cell structure of the Cr-doped Ge-core/Si-shell nanowire along the $\langle 110\rangle$ direction for various dopant configurations represented by I, II, III, IV, and V; the unsaturated surface states are passivated by H-atoms. (Reprinted with permission from Nano Lett. 2021, 21(4), 1856-1862. Copyright (2021) American Chemical Soci-

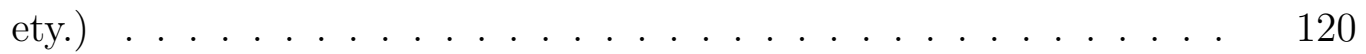


5.2 (a) Energy (relative to minimum energy configuration I) vs. Crconfigurations in the nanowire; the various configurations of the two Cr-atoms in the nanowire is represented by indices I, II, III, IV, and V (see Figure 5.1). (b) Energy vs. lattice parameter of the nanowire for configuration I. (c) Schematic showing the superexchange interaction mediated by the $p_{z}$ orbitals of the Ge that dictates the AFM spin ordering between $\operatorname{Cr}\left(d_{y z}\right.$-orbitals). (Reprinted with permission from Nano Lett. 2021, 21(4), 1856-1862. Copyright (2021) American Chemical

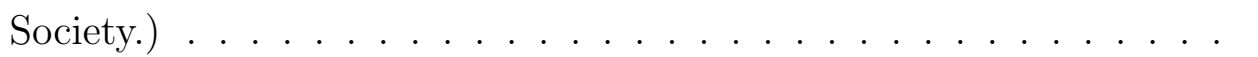

5.3 (a-c) Atom projected electronic band structure (PBE) of Cr-doped Gecore/Si-shell nanowire (configuration I) for the spin-up direction. The circle represents the contribution of the individual $\mathrm{Ge}$, Si, and $\mathrm{Cr}$ atoms to energy bands. The larger the diameter of the circle, the larger the contribution. The energy band diagrams for the spin-down electrons are not shown as the energy levels for spin-up and spin-down directions are degenerate for this AFM system. (Reprinted with permission from Nano Lett. 2021, 21(4), 1856-1862. Copyright (2021) American

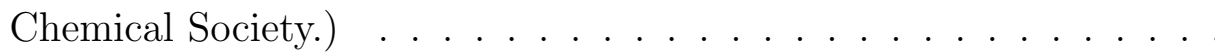


5.4 Total and orbital decomposed density of states (DOS) of Cr-doped Ge-core/Si-shell nanowire (configuration I) (i) total DOS in spin up and spin down directions (ii-iv) orbital decomposed DOS for the Ge, $\mathrm{Si}$, and $\mathrm{Cr}$ atoms respectively; the orbital decomposed DOS for the spin-down electrons are not shown as the energy levels for spin-up and spin-down directions are degenerate for this antiferromagnetic (AFM) system. (Reprinted with permission from Nano Lett. 2021, 21(4), 1856-1862. Copyright (2021) American Chemical Society.) . . . . 124

5.5 Plot showing the contribution of $\mathrm{d}_{\mathrm{xy}}, \mathrm{d}_{\mathrm{yz}}, \mathrm{d}_{\mathrm{xz}}, \mathrm{dz}^{2}$, and $\mathrm{dx}^{2}-\mathrm{y}^{2}$ orbitals of Cr to energy bands of Cr-doped Ge-core/Si-shell nanowire (configuration I). (Reprinted with permission from Nano Lett. 2021, 21(4), 1856-1862. Copyright (2021) American Chemical Society.) . . . . . 125

5.6 Plot showing contribution of $\mathrm{p}_{\mathrm{y}}, \mathrm{p}_{\mathrm{z}}$, and $\mathrm{p}_{\mathrm{x}}$ orbitals of Ge $(\mathrm{a}, \mathrm{b}$, and c) and Si (d, e, and f) to energy bands of Cr-doped Ge-core/Sishell nanowire (configuration I). (Reprinted with permission from Nano Lett. 2021, 21(4), 1856-1862. Copyright (2021) American Chemical Society.) ..................... 126 
5.7 Electronic band structure of Cr-doped Ge-core/Si-shell nanowire (configuration I) obtained using (a) hybrid functional, HSE06 (b) PBE functional with and without spin-orbit coupling (SOC). The bands for spin-up and spin-down directions are degenerate. (Reprinted with permission from Nano Lett. 2021, 21(4), 1856-1862. Copyright (2021) American Chemical Society. . . . . . . . . . . . . . . . . . . . 128

5.8 Variation of bandgap and exchange energy with strain in the Cr-doped Ge-core/Si-shell nanowire (configuration I). The strain in percentage is calculated as: $\operatorname{strain}(\%)=\left(\frac{a-a_{0}}{a_{0}}\right) \times 100 \%$, where $a_{0}$ is the equilibrium lattice parameter. (Reprinted with permission from Nano Lett. 2021, 21(4), 1856-1862. Copyright (2021) American Chemical Society.) .

5.9 Electronic band structure (PBE) of Cr-doped Ge-core/Si-shell nanowire (configuration I) at different values of (a-d) tensile $(+1.26 \%$, $+2.52 \%,+3.79 \%$, and $+5.05 \%)(\mathrm{e}-\mathrm{g})$ compressive $(-3.79 \%,-2.52 \%$, $1.26 \%$, and $0.00 \%$ ) strains. (h) represents the electronic band structure at $0.00 \%$ strain. The bands for spin-up and spin-down directions are degenerate. (Reprinted with permission from Nano Lett. 2021, 21(4), 1856-1862. Copyright (2021) American Chemical Society.) . . . . . 130 
5.10 Electronic band structure (PBE) of Cr-doped Ge-core/Si-shell nanowire (configuration I) for the (a) spin-up (b) spin-down direction at a compressive strain of $-5.05 \%$. (Reprinted with permission from Nano Lett. 2021, 21(4), 1856-1862. Copyright (2021) American Chemical Society. $\ldots \ldots \ldots \ldots \ldots \ldots \ldots$

5.11 (a) Current (I)-Voltage $\left(\mathrm{V}_{\mathrm{ds}}\right)$ characteristics in a two-terminal Crdoped Ge-core/Si-shell nanowire junction; spin-up $\left(\mathrm{I}_{\alpha}\right)$ and spin-down $\left(\mathrm{I}_{\beta}\right)$ currents are identical. (b) Comparison of I-V characteristics between undoped and Cr-doped nanowire junction. (Reprinted with permission from Nano Lett. 2021, 21(4), 1856-1862. Copyright (2021) American Chemical Society. . . . . . . . . . . . . . . . . . . . . .

5.12 (a-c) Bias-dependent spin-polarized transmission, $\mathrm{T}^{\alpha, \beta}(\mathrm{E}, \mathrm{V})$, as a function of injection energy in the Cr-doped core-shell nanowire junction. (d) Transmission as a function of injection energy for undoped nanowire junction. In all figures, the chemical potential windows (for integration) are represented by the dotted lines (red) . . . . . . 133

5.13 Projected atom decomposed spin-polarized density of states (DOS) for (a) undoped (b) Cr-doped nanowire junction; (c) is the magnified version of (b). (Reprinted with permission from Nano Lett. 2021, 21(4), 1856-1862. Copyright (2021) American Chemical Society.) . 135 
6.1 The unit cell structures of pristine (a) PbTe (b) PbS nanowire along the $\langle 200\rangle$ direction. Similarly, (c) and (d) illustrate the pristine $\mathrm{PbTe}$ and $\mathrm{PbS}$ nanowire unit cell structures in the $\langle 111\rangle$ direction. (Reprinted with permission from J. Phys. Chem. C https://doi.org/10.1021/acs.jpcc.1c06577. Copyright (2021) American Chemical Society. _.................. 141

6.2 The unit cell structures of (a) small core $\left(d_{c} \sim 6.32 \AA\right)$ (b) big core $\left(d_{c} \sim 12.82 \AA\right) \mathrm{PbTe} / \mathrm{PbS}$ core/shell nanowire along the $\langle 200\rangle$ direction. Similarly, (c) and (d) illustrate the small core $\left(d_{c} \sim 5.13 \AA\right)$ and big core $\left(d_{c} \sim 10.83 \AA\right) \mathrm{PbTe} / \mathrm{PbS}$ core/shell unit cell structures in the $\langle 111\rangle$ direction. (Reprinted with permission from J. Phys. Chem. C https://doi.org/10.1021/acs.jpcc.1c06577. Copyright (2021) American Chemical Society.) . . . . . . . . . . . . . . . . 142

6.3 The electronic band structure of bulk (a) PbS (b) PbTe obtained using PBE and PBE+SOC. (Reprinted with permission from J. Phys. Chem. C - https://doi.org/10.1021/acs.jpcc.1c06577. Copyright (2021) American Chemical Society.) . . . . . . . . . . . . . . . . . . . 143 
6.4 ( $\mathrm{a}$ and $\mathrm{b}$ ) The atom decomposed electronic band structure (PBE) of $\mathrm{PbTe}$ nanowire in the $\langle 200\rangle$ direction; the circle represents the contribution of the $\mathrm{Pb}$ and Te atoms to energy bands. The electronic band structures of $\mathrm{PbTe}$ nanowire obtained using $\mathrm{PBE}$ and $\mathrm{PBE}+\mathrm{SOC}$ are compared in (c). (Reprinted with permission from J. Phys. Chem. C https://doi.org/10.1021/acs.jpcc.1c06577. Copyright (2021) American Chemical Society.) . . . . . . . . . . . . . . . . . . . 144

6.5 ( $\mathrm{a}$ and $\mathrm{b}$ ) The atom decomposed electronic band structure (PBE) of $\mathrm{PbTe}$ nanowire in the $\langle 111\rangle$ direction; the circle represents the contribution of the $\mathrm{Pb}$ and $\mathrm{Te}$ atoms to energy bands. The electronic band structures of $\mathrm{PbTe}$ nanowire obtained using $\mathrm{PBE}$ and $\mathrm{PBE}+\mathrm{SOC}$ are compared in (c). (Reprinted with permission from J. Phys. Chem. C https://doi.org/10.1021/acs.jpcc.1c06577. Copyright (2021) American Chemical Society. $\ldots \ldots \ldots \ldots \ldots \ldots$

6.6 (a and b) The atom decomposed electronic band structure (PBE) of $\mathrm{PbS}$ nanowire in the $\langle 200\rangle$ direction; the circle represents the contribution of the $\mathrm{Pb}$ and $\mathrm{S}$ atoms to energy bands. The electronic band structures of $\mathrm{PbS}$ nanowire obtained using $\mathrm{PBE}$ and $\mathrm{PBE}+\mathrm{SOC}$ are compared in (c). (Reprinted with permission from J. Phys. Chem. C https://doi.org/10.1021/acs.jpcc.1c06577. Copyright (2021) American Chemical Society.) . . . . . . . . . . . . . . . . . 146 
6.7 ( $\mathrm{a}$ and $\mathrm{b}$ ) The atom decomposed electronic band structure (PBE) of $\mathrm{PbS}$ nanowire in the $\langle 111\rangle$ direction; the circle represents the contribution of the $\mathrm{Pb}$ and $\mathrm{S}$ atoms to energy bands. The electronic band structures of $\mathrm{PbS}$ nanowire obtained using $\mathrm{PBE}$ and $\mathrm{PBE}+\mathrm{SOC}$ are compared in (c). (Reprinted with permission from J. Phys. Chem. C https://doi.org/10.1021/acs.jpcc.1c06577. Copyright (2021) American Chemical Society.) . . . . . . . . . . . . . . . . . 147

6.8 The atom decomposed electronic band structure of $\mathrm{PbTe} / \mathrm{PbS}$ core/shell nanowires in the $\langle 200\rangle$ direction for (a-c) small core $\left(d_{c} \sim 6.32\right.$ $\AA)$ (e-f) big core $\left(d_{c} \sim 12.82 \AA\right)$ nanowire heterostructures; the circle represents the contribution of the $\mathrm{Pb}, \mathrm{Te}$, and $\mathrm{S}$ atoms to energy bands. The electronic band structures for small core and big core nanowire obtained using $\mathrm{PBE}$ and $\mathrm{PBE}+\mathrm{SOC}$ are compared in (d) and (h). (Reprinted with permission from J. Phys. Chem. C https://doi.org/10.1021/acs.jpcc.1c06577. Copyright (2021) American Chemical Society.) . . . . . . . . . . . . . . . . . . . . . 149 
6.9 The atom decomposed electronic band structure of $\mathrm{PbTe} / \mathrm{PbS}$ core/shell nanowires in the $\langle 111\rangle$ direction for $(\mathrm{a}-\mathrm{c})$ small core $\left(d_{c} \sim 5.13\right.$ $\AA)$ (e-f) big core $\left(d_{c} \sim 10.83 \AA\right)$ nanowire heterostructures; the circle represents the contribution of the $\mathrm{Pb}, \mathrm{Te}$, and $\mathrm{S}$ atoms to energy bands. The electronic band structures for small core and big core nanowire obtained using $\mathrm{PBE}$ and $\mathrm{PBE}+\mathrm{SOC}$ are compared in (d) and (h). (Reprinted with permission from J. Phys. Chem. C https://doi.org/10.1021/acs.jpcc.1c06577. Copyright (2021) American Chemical Society. $\ldots \ldots \ldots \ldots \ldots \ldots \ldots$

6.10 (a) Bandgap vs. Strain (\%) (b) Relative energy vs. Strain (\%) plots for $\mathrm{PbTe} / \mathrm{PbS}$ core/shell nanowires along the $\langle 200\rangle$ direction; (c) Bandgap vs. Strain (\%) (d) Relative energy vs. Strain (\%) plots for these heterostructures in the $\langle 111\rangle$ direction. Here, Relative energy $(E)=E_{\text {strain }}-E_{0}$, where $E_{\text {strain }}$ and $E_{0}$ are the energy of the strained and strain free nanowire. The strain (\%) is defined as: $\epsilon(\%)=\left(\frac{\mathrm{a}-\mathrm{a}_{0}}{\mathrm{a}_{0}}\right) \times 100 \%$, where $a_{0}$ is the lattice constant for the strain free nanowire. (Reprinted with permission from J. Phys. Chem. C https://doi.org/10.1021/acs.jpcc.1c06577. Copyright (2021) American Chemical Society.) . . . . . . . . . . . . . . . . 153 
6.11 Bandgap vs. Strain (\%) plot for $\mathrm{PbTe} / \mathrm{PbS}$ core/shell nanowires in $(\mathrm{a})\langle 200\rangle$ (b) $\langle 111\rangle$ direction. SOC is included in these calculations. (Reprinted with permission from J. Phys. Chem. C https://doi.org/10.1021/acs.jpcc.1c06577. Copyright (2021) American Chemical Society.) . . . . . . . . . . . . . . . . . 154

6.12 The energy dependence of the real $\left(\epsilon_{1}(\omega)\right)$ part of $\epsilon(\omega)$, imaginary $\left(\epsilon_{2}(\omega)\right)$ part of $\epsilon(\omega)$, and absorption coefficient $(\alpha)$ for (a-c) small core (d-f) big core nanowire in the $\langle 200\rangle$ direction. No SOC is included in these calculations. (Reprinted with permission from J. Phys. Chem. C - https://doi.org/10.1021/acs.jpcc.1c06577. Copyright (2021) American Chemical Society.) . . . . . . . . . . . . . .

6.13 Atom and orbital decomposed electronic density of states (DOS) of small core $\mathrm{PbTe} / \mathrm{PbS}$ nanowire in the $\langle 200\rangle$ direction. No SOC is included in these calculations. (Reprinted with permission from J. Phys. Chem. C - https://doi.org/10.1021/acs.jpcc.1c06577. Copyright (2021) American Chemical Society.) . . . . . . . . . . . . . . .

6.14 Atom and orbital decomposed electronic density of states (DOS) of big core $\mathrm{PbTe} / \mathrm{PbS}$ nanowire in the $\langle 200\rangle$ direction. No SOC is included in these calculations. (Reprinted with permission from J. Phys. Chem. C - https://doi.org/10.1021/acs.jpcc.1c06577. Copyright (2021) American Chemical Society.) . . . . . . . . . . . . . 158 
6.15 The energy dependence of the real $\left(\epsilon_{1}(\omega)\right)$ part of $\epsilon(\omega)$, imaginary $\left(\epsilon_{2}(\omega)\right)$ part of $\epsilon(\omega)$, and absorption coefficient $(\alpha)$ for (a-c) small core (d-f) big core nanowire in the $\langle 111\rangle$ direction. No SOC is included in these calculations. (Reprinted with permission from J. Phys. Chem. C - https://doi.org/10.1021/acs.jpcc.1c06577. Copyright (2021) American Chemical Society.) . . . . . . . . . . . . . . .

6.16 The energy dependence of the real $\left(\epsilon_{1}(\omega)\right)$ part of $\epsilon(\omega)$, imaginary $\left(\epsilon_{2}(\omega)\right)$ part of $\epsilon(\omega)$, and absorption coefficient $(\alpha)$ for (a-c) small core (d-f) big core nanowire in the $\langle 200\rangle$ direction. SOC is included in these calculations. (Reprinted with permission from J. Phys. Chem. C https://doi.org/10.1021/acs.jpcc.1c06577. Copyright (2021) American Chemical Society.) . . . . . . . . . . . . . . . .

6.17 The energy dependence of the real $\left(\epsilon_{1}(\omega)\right)$ part of $\epsilon(\omega)$, imaginary $\left(\epsilon_{2}(\omega)\right)$ part of $\epsilon(\omega)$, and absorption coefficient $(\alpha)$ for (a-c) small core (d-f) big core nanowire in the $\langle 111\rangle$ direction. SOC is included in these calculations. (Reprinted with permission from J. Phys. Chem. C https://doi.org/10.1021/acs.jpcc.1c06577. Copyright (2021) American Chemical Society.) . . . . . . . . . . . . . 161 
6.18 Cohesive energy of the pristine and core/shell nanowires along the $\langle 200\rangle$ and $\langle 111\rangle$ directions; the stability of nanowires in the $\langle 111\rangle$ direction is relatively less compared to that in $\langle 200\rangle$ direction. (Reprinted with permission from J. Phys. Chem. C https://doi.org/10.1021/acs.jpcc.1c06577. Copyright (2021) American Chemical Society.) . . . . . . . . . . . . . . . . . . 162

6.19 Temperature vs. Time ((a) \& (b)) and Energy vs. Time ((c) \& (d)) plot for small core nanowires oriented in $\langle 200\rangle$ and $\langle 111\rangle$ directions; the partial $(\mathrm{Pb}-\mathrm{Te})$ radial distribution function $(\mathrm{RDF})$ is shown in (e) and (f). (Reprinted with permission from J. Phys. Chem. C https://doi.org/10.1021/acs.jpcc.1c06577. Copyright (2021) American Chemical Society.) . . . . . . . . . . . . . . . . . . 164

6.20 Temperature vs. Time ((a) \& (b)) and Energy vs. Time ((c) \& (d)) plot for big core nanowires oriented in $\langle 200\rangle$ and $\langle 111\rangle$ directions; the partial $(\mathrm{Pb}-\mathrm{Te})$ radial distribution function $(\mathrm{RDF})$ is shown in (e) and (f). (Reprinted with permission from J. Phys. Chem. C https://doi.org/10.1021/acs.jpcc.1c06577. Copyright (2021) American Chemical Society.) . . . . . . . . . . . . . . . . . 165 



\section{Preface}

All the results presented in this thesis are published in peer review journals. Chapter 4 of this thesis is published in Nanoscale Advances (Nanoscale Adv. 2020, 2, 1843-1849). Chapter 5 is published in Nano Letters (Nano Lett. 2021, 21(4), 1856-1862). Chapter 6 is published in Journal of Physical Chemistry Chttps://doi.org/10.1021/acs.jpcc.1c06577. All the three projects were defined by my advisor, Prof. Ranjit Pati. Calculations were done by me under his supervision. All results were analysed by both of us and both of us worked on the paper. In the Cr-

doped Ge-core/Si-shell project published in Nano Letters (Nano Lett. 2021, 21(4), 1856-1862), Dr. Durga Paudyal from Ames Lab provided useful suggestions. 



\section{Acknowledgments}

First, I would like to give a sincere thank to my advisor Prof. Ranjit Pati for defining my Ph.D. projects, valuable time and effort he has put in supervising and improving me as a researcher, and constant support during my Ph.D. I am honored to have him as my research supervisor. I will remain indebted to him for the knowledge and expertise I gained under his supervision.

I would also like to give a sincere thank to Prof. Ravindra Pandey, the chair of physics department at MTU, for his valuable suggestions, guidance, and unconditional support during these three years at MTU.

I am extremely grateful to my Ph.D. committee members Prof. Ranjit Pati, Prof. Ravindra Pandey, Prof. Maximilian J. Seel, and Prof. Susanta Ghosh for serving in my Ph.D. committee, the valuable time spent for critically revising my thesis, useful feedback for improvements, and writing recommendation letters for me.

I am also thankful to Prof. Narayan Adhikari for writing letters of recommendation for me, especially to MTU. I would also like to thank him for his support whenever needed and everything that I learned from him.

I am highly grateful to the Graduate School and Dean Awards Advisory Committee 
at MTU for supporting me through the doctoral finishing fellowship to complete my dissertation during the summer (2021) semester. I am also grateful to Elizabeth and Richard Henes Center for Quantum Phenomena and it's director Prof. Jacek Borysow for providing me the financial support through Henes fellowship for the Fall 2021.

I am also thankful to the faculties, friends/colleagues, and staffs of the physics department at MTU for the help that I got from them. In addition, thanks to the IT (MTU) for providing instant help whenever needed and Dr. Gowtham, Director of research computing, IT for providing the LATEX template to compile this thesis. Furthermore, I would like to thank our collaborator Dr. Durga Paudyal from Ames lab for his suggestions in the Cr-doped nanowire project and Dr. Meghnath Jaishi for his help during initial days of my Ph.D.

My sincere gratitude also extends to Dr. Utsab Shrestha, Dr. Gajhadhar Joshi, Dr. Rabi Khanal, Mr. Raju KC, and Dr. Suman Dhamala for their encouragements and fruitful discussions.

Finally, I am extremely grateful to my family members for the unconditional love and support that they have shown all the time. 


\section{Abstract}

Nanoscale systems, especially the one-dimensional semiconducting nanowires, have been the subject of immense research interests due to their potential applications in nanoelectronics and optoelectronics that demand cheaper, smaller, faster, and energyefficient components. In particular, the core/shell nanostructures, in which the core materials are shielded by materials with larger bandgap called shell, have been shown to enhance the performance of field effect transistors (FETs), solar cells, light emitting diodes (LEDs), and thermoelectric devices due to their outstanding features like valence band offset between the core and shell, higher stability against oxidation, reduction in the surface trap states, diminished nonradiative recombination processes, and enhancement in the carrier multiplication and carrier transport processes. Incorporation of spin functionality via doping of a magnetic impurity into such core/shell (non-magnetic) nanostructures also offers additional advantages for next-generation spin-based electronic devices. Such devices are not only smaller, cost-effective, and non-volatile but also have increased data processing speed, consume less power, and assist reducing heat dissipation compared to the traditional electronic devices. In the first part of my thesis, I have studied the role of $\mathrm{Mn}$ and $\mathrm{Cr}$ dopants on the electronic structure, magnetic properties, and strain-induced magnetic phase transitions in $\mathrm{Ge} / \mathrm{Si}$ core/shell nanowire heterostructures using the many-body density functional theory (DFT) approach. Subsequently, I have designed a spin filtering device using 
Mn-doped Ge/Si core/shell nanowire and a switching device using Cr-doped $\mathrm{Ge} / \mathrm{Si}$ core/shell nanowire. To understand the spin-transport properties of these devices, I have used a real space orbital based DFT in conjunction with the single-particle non-equilibrium Green's function approach. In the second part of my thesis, I have studied the effect of size and growth direction on the electronic structure, stability, mechanical, and optical properties for $\mathrm{PbTe} / \mathrm{PbS}$ core/shell nanowires. To understand the thermodynamic stability of these complex structures, I have performed the ab-initio molecular dynamics simulations that demonstrate the possibilities of core-to-shell diffusion at room temperature in certain growth direction. 


\section{Chapter 1}

\section{Introduction}

Since the invention of the first transistor in 1947 by John Bardeen and Walter Brattain, followed by the development of the first integrated circuit in 1958 by Jack Kilby, the ability to fabricate transistors with reduced size and fit billions of them in a microprocessor chip leading to much faster, lighter, smaller and cheaper electronic devices that consume less power has revolutionized the field of solid-state-circuits industry [1, 2]. The steady rise in device miniaturization in the last few decades was foreseen from Moore's law, initially proposed by Gordan Moore in 1965, which states the number of transistors in a microprocessor chip and its performance will double every two years [1, 3, 4]. Since then, Moore's law has been the guiding principle for the exponential growth of the technological advancement that has transformed the less sophisticated machines of the 1970s and 1980s to modern-day smartphones, laptops, 
automated devices, and high-speed internet[1]. Until 2000s, this simple principle of shrinking down the size of the transistors to make them much faster, leading to an unprecedented increase in the performance of electronic devices, worked well [1, [5]. But, this was expected to last for a finite time. In the early 2000s, when the size of the transistor was reduced to $\sim 90 \mathrm{~nm}$, the chips started to get hotter due to the rapid movement of the electrons in the circuit[1]. To avoid this technological challenge, the clock rates, which measure the microprocessor's ability to process instructions, were kept fixed[1]. To follow Moore's law for technological advancement with the restriction on the electrons speed, machines with multi-cores in a chip were designed[1]. Now, the size of the modern-day transistor is already in the path of reaching its physical limits. At such atomic scales, the quantum effects dominate, leading to the tunneling (quantum) of the electrons through the insulating layers. This makes the performance of the transistor extremely unreliable, and the transistor will no longer act as a switch [1]. To overcome this hurdle in the near future and continue the scaling of transistors size, researchers are in search of alternative approaches that can make the device's size smaller, faster, and consume less power than the existing siliconbased complementary metal-oxide-semiconductor (CMOS) technology. This has led to the discovery of several low-dimensional systems.

Low-dimensional systems [6, 7, 8, 9, 10, 11, 12, 13] are the ones in which the motion of quantum mechanical particles like electrons or holes are confined in one or more directions. Such systems can be formed by the reduction of the size of 
the bulk system in one or more dimensions[14]; the length scale ( $\sim$ few nanometers) of such systems lie within the characteristic length scale of an atom and the host (bulk) material[15]. These systems can be 2-d nanosheets[16, 17], 1-d nanowires/nanotubes [18, 19, 20, 21, 22, 23] and 0-d quantum dots[6]. These materials offer wide range of possibilities from topological insulators [24, 25, 26] to insulators[27, 28, 29] to semiconductors[18, 19, 30, 31] to semi-metals[16, 32] to metals [33, 34] to superconductors [35, 36]. In 1-d nanowires [7, 8, 9, 10], which is the interest of this work, the carriers are confined in two transverse directions, but are free to move along the nanowire axis. Here, we are interested in the core-shell nanowire heterostructures [8, 18, 19, 37, 38, 39, 40, 41, 42], especially the Ge/Si core/shell and $\mathrm{PbTe} / \mathrm{PbS}$ core/shell nanowires, in which the core material (Ge in $\mathrm{Ge} / \mathrm{Si}$ core/shell and $\mathrm{PbTe}$ in $\mathrm{PbTe} / \mathrm{PbS}$ core/shell nanowire) is shielded by a material with higher bandgap called shell ( $\mathrm{Si}$ in $\mathrm{Ge} / \mathrm{Si}$ core/shell and $\mathrm{PbS}$ in $\mathrm{PbTe} / \mathrm{PbS}$ nanowires). Such geometry in $\mathrm{Ge} / \mathrm{Si}$ core/shell nanowire leads to the valence band offset $\sim 0.5 \mathrm{eV}$ between the Ge-core and Si-shell[8], thus minimizing the effect of the Schottky barrier to the device performance[8]. It is worth noting that the formation of the Schottky barrier limits the performance of the nanoelectronic devices in the homogenous Si and Ge nanowires [37]. Subsequently, a low bias ballistic transport with scattering mean free path of $\sim 500 \mathrm{~nm}\left[19\right.$, 37] and high carrier (hole) mobility of $\sim 730 \mathrm{~cm}^{2} \mathrm{~V}^{-1} \mathrm{~s}^{-1}$ ( $\sim$ factor of 10 times larger than p-type Si metal oxide semiconducting field effect transistors (MOSFETs)) [37] have been reported in these nanowire heterostructures, 
leading to the superior performance of the field effect transistors (FETs) compared to the current state of the art MOSFETs[37]. The programmable logic circuits of Ge/Si core/shell nanowire FETs have also been designed[43]. Moreover, the Ge/Si core/shell nanowires are compatible with the current Si-based technology and can be synthesized in high yield[18, 19, 37] with reproducible electronic properties.

Incorporating spin functionality in these radial heterostructures via doping of magnetic atoms that provides immunity from the substrate effect would offer an additional opportunity for using them in next-generation spintronics [44, 45, 46, 47, 48]. By exploiting the electron spin instead of its charge, the device can not only be made smaller, cost-effective, and non-volatile, but also have increased data processing speed, consume less power, and reduce heat dissipation compared to the traditional electronic devices. In this thesis, we first explored the role of $\mathrm{Mn}$ dopant to the electronic, magnetic, mechanical, and spin-transport properties of $\mathrm{Ge} / \mathrm{Si}$ core/shell nanowires. Numerous studies of Mn dopants in homogenous Si and Ge nanowires [49, 50, 51, 52, 53, 54, 55, 56] have been reported confirming the occurence of ferromagnetism at room temperature. However, the stabilization of the ferromagnetic phase at room temperature in homogenous Ge and Si nanowires can be a challenge due to the effect of the substrate. The core-shell geometry of these nanowires overcome such limitations by allowing one to dope the magnetic atoms in the core region of these nanowires. Due to the valence band offset between the $\mathrm{Ge}$ and $\mathrm{Si}$, 
the spin carriers in such structures can then be transported through the core, minimizing the spin lifetime degradation due to scattering and recombination with the surface states, as well as diminish the momentum-dependent randomization of spins (spin dephasing) [45]. In the first project, using first-principles density functional theory that does not make any assumptions of the electronic structure, we investigated the role of small concentration of $\mathrm{Mn}$ dopants $(\leq 2 \%)$ to the electronic, magnetic, and mechanical properties of Ge/Si core/shell nanowire heterostructures. For understanding the usage of this newly tailored material in a practical device settings, we studied the spin-transport properties of a prototypical nanowire (magnetic) junction using DFT and the single particle non-equilibrium Green's function approach. Based on our study, we report that Mn doped nanowire (Ge/Si core/shell) can act as an excellent spin filter with spin filtering efficiency of $90.4 \%$.

Having shown that the substitutional doping of Mn into the Ge-core/Si-shell nanowire transforms the semiconducting material to a ferromagnetic half-metal[57], the possibility of antiferromagnetic (AFM) semiconducting behavior with Cr-dopant is explored in the second project. An antiferromagnet offers many important functionalities such as opportunities for electrical control of magnetic domains, immunity from magnetic perturbations, and fast spin dynamics [58, 59, 60, 61, 62, 63, 64, 65, 66]. Introducing some of these intriguing features of an antiferromagnet into a low dimensional semiconductor core-shell nanowire offers an exciting pathway for its usage in 
antiferromagnetic semiconductor spintronics [59, 60, 61, 62]. Using a quantum mechanical DFT approach, we predict that the Cr-doped Ge-core/Si-shell nanowire is an antiferromagnetic semiconductor. The spin polarized transport calculations using a finite cluster real space DFT together with the non equilibrium Green's function approach reveal that this material can be used as an electrical switch with a high ON/OFF current ratio ( $\sim 41$ times higher for the $\mathrm{ON}$ state at a relatively small bias of $0.83 \mathrm{~V})$.

On the other hand, in the $\mathrm{PbTe} / \mathrm{PbS}$ core/shell heterostructure, the shelling of the $\mathrm{PbTe}$ nanostructure with $\mathrm{PbS}$ suppresses the hole conductivity by localizing it within the nanocrystal and enhances the electron conductivity, making the carrier transport completely n-type (unipolar) incontrary to the ambipolar transport of carriers in pristine $\mathrm{PbTe}$ and $\mathrm{PbS}$ nanostructures. This also means that these core/shell heterostructres are suitable for photodetectors, thermoelectric and electron transport layer in photovoltaic devices[42]. Furthermore, it has been observed that the shelling enhances the stability of nanocrystals against oxidation [67], reduces the trap states, diminishes the non-radiative recombination processes [68, and enhances the carrier multiplication [69] and (carrier) transport processes [70]. The successful synthesis of nanocrystals of lead chalcogenides $42,71,72,73,74,75]$ accompanied by the ability to tune the thickness of the core and shell provides additional opportunities for studying 
the tunable electronic properties of these quantum materials for a wide range of applications including but not limited to FETs[42, 76, 77], solar cells[78], LEDs[42, highperformance optoelectronics [79, 80], and high-efficiency thermoelectrics[72]. Therefore, in the third project, we studied the directional dependence, as well as the effect of size of the core to the electronic structure, thermodynamical stability, mechanical, and optical properties of the $\mathrm{PbTe} / \mathrm{PbS}$ core/shell nanowire heterostructures using DFT and ab intio molecular dynamics.

This thesis is organized as follows. I have discussed in brief the many-body density functional theory and quantum transport in a nanoscale junction in Chapter 2 and Chapter 3, respectively. The results of three projects mentioned here are included in Chapter 4, Chapter 5, and Chapter 6 of this thesis followed by conclusion and future work in Chapter 7. 



\section{Chapter 2}

\section{Many-Body Theory}

\subsection{Many-Body Schrödinger Wave Equation}

In this chapter, I will discuss the many-body theory that has been used to study the electronic, magnetic, mechanical, and optical properties of the core-shell nanowire heterostructures. The theory of quantum transport will be discussed in Chapter 3 . Let's begin by writing the non-relativistic time-independent many-body Schrödinger wave equation (SWE) [81, 82]

$$
\hat{H} \Psi(\{\mathbf{r}\},\{\mathbf{R}\})=E \Psi(\{\mathbf{r}\} ;\{\mathbf{R}\})
$$


In Equation 2.1. $\hat{H}$ is the Hamiltonian operator, E is the total energy, and $\Psi(\{\mathbf{r}\} ;\{\mathbf{R}\})$ is the total wave function of a many-body system that depends on the coordinates of all the electrons $\{\mathbf{r}\}$ and ions $\{\mathbf{R}\}$. The non-relativistic Hamiltonian $(\hat{H})$ for a system of $N_{e}$ electrons and $N_{I}$ ions in atomic units $\left(\hbar=m_{e}=e=1\right)$ can be expressed as $81,82,83]$

$$
\begin{aligned}
\hat{H} & =-\frac{1}{2} \sum_{i=1}^{N_{e}} \nabla_{i}^{2}-\frac{1}{2} \sum_{I=1}^{N_{I}} \frac{\nabla_{I}^{2}}{M_{I}}-\sum_{i=1}^{N_{e}} \sum_{I=1}^{N_{I}} \frac{Z_{I}}{\left|\mathbf{r}_{i}-\mathbf{R}_{I}\right|} \\
& +\frac{1}{2} \sum_{i=1}^{N_{e}} \sum_{j \neq i}^{N_{e}} \frac{1}{\left|\mathbf{r}_{i}-\mathbf{r}_{j}\right|}+\frac{1}{2} \sum_{I=1}^{N_{I}} \sum_{J \neq I}^{N_{I}} \frac{Z_{I} Z_{J}}{\left|\mathbf{R}_{I}-\mathbf{R}_{J}\right|}
\end{aligned}
$$

In Equation 2.2, $m_{e}$ is the mass of an electron, $Z_{I}$ and $M_{I}$ are the atomic number and mass of $I^{\text {th }}$ ion, $\mathbf{r}_{i}$ and $\mathbf{r}_{j}$ are position of $i^{\text {th }}$ and $j^{\text {th }}$ electron, and $\mathbf{R}_{I}$ and $\mathbf{R}_{J}$ are position of $I^{t h}$ and $J^{t h}$ ion. The first and the second terms in Equation 2.2 are the kinetic energy operators for the electrons and ions respectively, the third term is the potential energy of attraction between the electrons and the ions, the fourth term is the potential energy of repulsion between the electrons, and the fifth term is the potential energy of repulsion between the ions. The factor $\frac{1}{2}$ in the fourth and fifth terms arises to avoid counting of the same interaction twice in the Hamiltonian. It may be noted that in writing the Hamiltonian (Equation 2.2), the spin-orbit interactions are not taken into account. 


\subsection{Born-Oppenheimer Approximation}

At this point, there are two major challenges in solving Equation 2.1: (i) the electronelectron interaction terms in the Hamiltonian are not separable (ii) the wave function of the many-body system depends on the positions of all the electrons and ions, implying that Equation 2.1 is a coupled problem of the electrons and ions. Therefore, it can't be solved without some approximations. Born-Oppenheimer approximation 84 splits the complex problem of the many-body system involving electrons and ions into two separate problems: one for the electrons and the other for the ions. Since the mass of an ion is much larger than an electron, its kinetic energy is much smaller than the kinetic energy of an electron. Therefore, ions can be assumed to be at rest and the electrons adjust their coordinates instantaneously for a given ionic configuration. The Born-Oppenheimer approximation allows us to decouple the total wave function in Equation 2.1 as 81,82

$$
\Psi(\{\mathbf{r}\} ;\{\mathbf{R}\})=\Phi(\{\mathbf{r}\} ;\{\mathbf{R}\}) \zeta(\{\mathbf{R}\})
$$

Let's rewrite the Hamiltonian in Equation 2.2 as

$$
\hat{H}=\hat{T}_{e}+\hat{T}_{N}+\hat{V}_{e N}+\hat{V}_{e e}+\hat{V}_{N N}
$$


The many-body SWE (Equation 2.1) can then be split into the following two equations [81, 82]:

$$
\begin{gathered}
\left(\hat{T}_{e}+\hat{V}_{e N}+\hat{V}_{e e}+\hat{V}_{N N}\right) \Phi(\{\mathbf{r}\} ;\{\mathbf{R}\})=E_{n}^{t o t}(\{\mathbf{R}\}) \Phi(\{\mathbf{r}\} ;\{\mathbf{R}\}) \\
\left(\hat{T}_{N}(\{\mathbf{R}\})+E_{n}^{t o t}(\{\mathbf{R}\}) \zeta(\{\mathbf{R}\})=E_{n}^{n u c} \zeta(\{\mathbf{R}\})\right.
\end{gathered}
$$

The term $\hat{V}_{N N}$ in Equation 2.5 is a constant for a given ionic configuration and can be dropped. Let's rewrite Equation 2.5 as [81, 82]

$$
\begin{array}{r}
\left(\hat{T}_{e}+\hat{V}_{e N}+\hat{V}_{e e}\right) \Phi(\{\mathbf{r}\} ;\{\mathbf{R}\})=E_{n}^{e l}(\{\mathbf{R}\}) \Phi(\{\mathbf{r}\} ;\{\mathbf{R}\}) \\
\Rightarrow \hat{H}^{e l} \Phi(\{\mathbf{r}\} ;\{\mathbf{R}\})=E_{n}^{e l}(\{\mathbf{R}\}) \Phi(\{\mathbf{r}\} ;\{\mathbf{R}\})
\end{array}
$$

Equation 2.7 is now a complete electronic problem. The ionic coordinates $\{\mathbf{R}\}$ appears only as a parameter in Equation 2.7 and can be solved for different configurations of the nuclear coordinates to obtain the wave function $\Phi(\{\mathbf{r}\},\{\mathbf{R}\})$ and the energy $E_{n}^{e l}(\{\mathbf{R}\})$ of the many-electron system [81, 82. n, in Equation 2.7, is a quantum number that gives various energy levels of an electronic problem for a given configuration of ions $\{\mathbf{R}\}$. Equation 2.6 describes the rotational, vibrational, and translational motions of ions in potential energy surface (PES) obtained by solving Equation 2.5. A typical PES is shown in Figure 2.1[81]. In electronic structure, we are only interested in solving Equation 2.7 at the equilibrium ionic configuration. The 


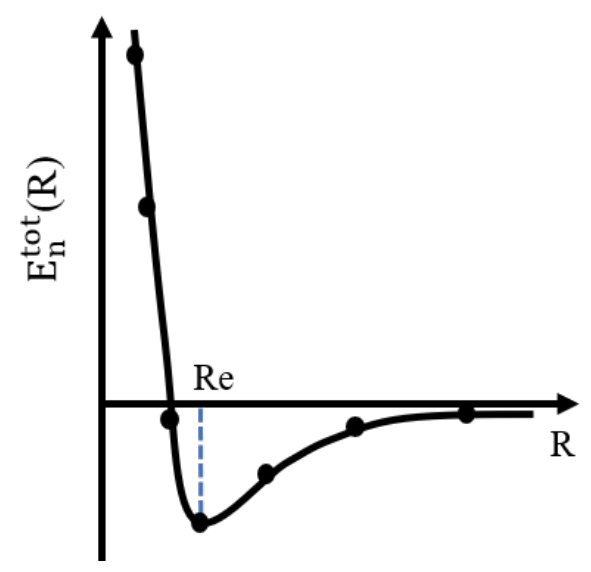

Figure 2.1: An example of potential energy curve for the ground state (GS) obtained by solving Equation 2.5, $R_{e}$ is the equilibrium configuration of ions.

complete form of Equation 2.7 is

$$
\begin{array}{r}
{\left[-\frac{1}{2} \sum_{i=1}^{N_{e}} \nabla_{i}^{2}-\sum_{i=1}^{N_{e}} \sum_{I=1}^{N_{I}} \frac{Z_{I}}{\left|\mathbf{r}_{i}-\mathbf{R}_{I}\right|}+\frac{1}{2} \sum_{i=1}^{N_{e}} \sum_{j \neq i}^{N_{e}} \frac{1}{\left|\mathbf{r}_{i}-\mathbf{r}_{j}\right|}\right] \Phi(\{\mathbf{r}\} ;\{\mathbf{R}\})} \\
=E(\{\mathbf{R}\}) \Phi(\{\mathbf{r}\} ;\{\mathbf{R}\})
\end{array}
$$

In Equation 2.8, $E_{n}^{e l}(\{\mathbf{R}\})$ is replaced by $E(\{\mathbf{R}\})$. Thus, the Born-Oppenheimer approximation simplifies the problem of a many-body system to some extent by splitting a single problem of electrons and ions into two problems that describe the motion of ions and electrons separately. But, even after this simplification, the solution to the many-body system remains challenging as the electron-electron interaction terms in the Hamiltonian (Equation 2.8) is unknown and inseparable. Therefore, we need to make approximations to decompose a single problem of $N_{e}$ electrons (Equation 2.8) to $N_{e}$ single electron problems. 


\subsection{Hellmann-Feynman Theorem}

Since we intend to solve Equation 2.8 at the equilibrium configuration of the ions, one may ask: how do we get the equilibrium geometry of the ions. For this, we use Hellmann-Feynmann theorem [85, 86] that provides a way to calculate the forces on atoms from the ground state energy. Since the ions move in the potential energy surface described by the electrons, the force acting on an atom I at position $R_{I}$ is given by the derivative of the total ground state energy of the electronic problem 87 ]

$$
F_{I}=-\frac{d E}{d R_{I}}
$$

The displacement of the ions cause the single electron orbitals (Kohn-Sham states to be discussed later) to change, giving rise to forces on ions [87]. These forces can be observed by writing Equation 2.9 as [87]

$$
F_{I}=-\frac{\partial E}{\partial R_{I}}-\sum_{i} \frac{\partial E}{\partial \phi_{i}} \frac{\partial \phi_{i}}{\partial R_{I}}-\sum_{i} \frac{\partial E}{\partial \phi_{i}^{*}} \frac{\partial \phi_{i}^{*}}{\partial R_{I}}
$$

If $\phi_{i}$ is an eigen state of the Hamiltonian $H, H \phi_{i}=\epsilon_{i} \phi_{i}$. The sum of second and third term in Equation 2.10 becomes zero if $\left\langle\phi_{i} \mid \phi_{i}\right\rangle=1$. Therefore, the force that an ion feels can be expressed as the partial derivative of the total energy with respect to 
the ionic position as

$$
F_{I}=-\frac{\partial E}{\partial R_{I}}
$$

This is called Hellmann-Feynmann theorem. It may be noted that the HellmannFeynmann forces are extremely sensitive to the errors in the electronic orbitals [87]. Therefore, these orbitals have to be relaxed close to their ground state for each ionic step as errors in these orbitals cause force on the atoms that prevent them from reaching the equilibrium ionic configuration[87]. In our calculations, we have assumed the residual forces acting on atoms to be less than a predefined value $(0.01 \mathrm{eV} / \AA)$ so that the Hellmann-Feynmann forces on them are negligible.

\section{$2.4 \quad$ Hartree-Fock Theory}

In Quantum Mechanics, there are two approaches to solve the problem of a manyelectron system: (i) Wave Function Theory (WFT) (ii) Density Functional Theory (DFT). Let's begin with discussing the WFT. Here, we limit our discussions of WFT to Hartree and Hartree-Fock Theory. Hartree Theory [88, 89, 90] is the simplest form of the WFT in which it is assumed that each electron moves in an effective field due to all the other $N_{e}-1$ electrons and the ions; their motion in such an effective field can be described by the single-particle SWE[91]. The $N_{e}$ electrons, in an effective 
potential, can be treated as independent and therefore the total wave function can be expressed as the product of individual orbitals for $N_{e}$ electrons as 91$]$

$$
\Phi\left(\mathbf{r}_{1}, \mathbf{r}_{2}, . ., \mathbf{r}_{i}, . ., \mathbf{r}_{N_{e}}\right)=\phi_{1}\left(\mathbf{r}_{1}\right) \phi_{2}\left(\mathbf{r}_{2}\right) \ldots \phi_{i}\left(\mathbf{r}_{i}\right) \ldots \phi_{N_{e}}\left(\mathbf{r}_{N_{e}}\right)
$$

Slater 92] and Fock [93] found that wave function in Equation 2.12 does not obey the correct symmetry of the wave function of the electrons and fails to capture the quantum mechanical phenomena like exchange interactions in materials. Therefore, we need Hartree-Fock theory $[94$, 95, 96]. Since electrons are fermions, the total wave function should be anti-symmetric i.e.

$$
\Phi\left(\mathbf{x}_{1}, \mathbf{x}_{2}, . ., \mathbf{x}_{i}, \mathbf{x}_{j}, . ., \mathbf{x}_{N_{e}}\right)=-\Phi\left(\mathbf{x}_{1}, \mathbf{x}_{2}, . ., \mathbf{x}_{j}, \mathbf{x}_{i}, . ., \mathbf{x}_{N_{e}}\right)
$$

An anti-symmetric wave function can be expressed in the form of Slater determinant [81, 97] as

$$
\Phi\left(\mathbf{x}_{1}, . ., \mathbf{x}_{i}, \mathbf{x}_{j}, . ., \mathbf{x}_{N}\right)=\frac{1}{\sqrt{N !}}\left|\begin{array}{cccc}
\phi_{1}\left(\mathbf{x}_{1}\right) & \phi_{2}\left(\mathbf{x}_{1}\right) & . . & \phi_{N_{e}}\left(\mathbf{x}_{1}\right) \\
\phi_{1}\left(\mathbf{x}_{2}\right) & \phi_{2}\left(\mathbf{x}_{2}\right) & . . & \phi_{N_{e}}\left(\mathbf{x}_{2}\right) \\
. . & . . & . . & . . \\
\phi_{1}\left(\mathbf{x}_{N_{e}}\right) & \phi_{2}\left(\mathbf{x}_{N_{e}}\right) & . . & \phi_{N_{e}}\left(\mathbf{x}_{N_{e}}\right)
\end{array}\right|
$$

In the above determinant (Equation 2.14), $\frac{1}{\sqrt{N !}}$ is the normalization constant and $\left(\phi_{1}, \phi_{2}, \phi_{3}, \ldots \ldots, \phi_{N_{e}}\right)$ represents the $N_{e}$ spin orbitals that are occupied by $N_{e}$ 
electrons [81]; the columns and rows are labelled by spin orbitals and electrons respectively[81]. This determinant is zero if any two columns are identical, suggesting that there cannot be two electrons occupying the same spin-orbital. This is Pauli's exclusion principle[81]. Furthermore, the determinant changes sign if any two rows are interchanged. This means the determinant preserves the anti-symmetry property of the many-electron wave function as interchanging two rows is equivalent to interchanging the coordinates of two particles. The total electronic Hamiltonian in Equation 2.8 can be expressed as the sum of the individual Hamiltonians for $N_{e}$ electrons as 91,95$]$

$$
\hat{H}=\sum_{i=1}^{N_{e}}\left[\hat{h}\left(\mathbf{r}_{i}\right)+\hat{w}\left(\mathbf{r}_{i}, \mathbf{r}_{j}\right)\right]
$$

In Equation 2.15, $\hat{h}\left(\mathbf{r}_{i}\right)$ and $\hat{w}\left(\mathbf{r}_{i}, \mathbf{r}_{j}\right)$ are the one and two electron operators and are given by

$$
\begin{gathered}
\hat{h}\left(\mathbf{r}_{i}\right)=-\frac{1}{2} \nabla_{i}^{2}-\sum_{I=1}^{N_{I}} \frac{Z_{I}}{\left|\mathbf{r}_{i}-\mathbf{R}_{I}\right|}=-\frac{1}{2} \nabla_{i}^{2}+V_{e x t}\left(\mathbf{r}_{i}\right) \\
\hat{w}\left(\mathbf{r}_{i}, \mathbf{r}_{j}\right)=\frac{1}{2} \sum_{j \neq i}^{N_{e}} \frac{1}{\left|\mathbf{r}_{i}-\mathbf{r}_{j}\right|}
\end{gathered}
$$

In Hartree-Fock theory, we take the best possible single Slater determinant to represent the ground state of a many-electron system. The best possible determinant is 
obtained by minimization of the energy with respect to these orbitals. The expectation value of the Hamiltonian in Equation 2.15 can be expressed as 91]

$$
\begin{aligned}
E & =\langle\Phi|\hat{H}| \Phi\rangle \\
& =\sum_{i=1}^{N_{e}} h_{i}+\frac{1}{2} \sum_{i=1}^{N_{e}} \sum_{j=1}^{N_{e}}\left(J_{i j}-K_{i j}\right)
\end{aligned}
$$

The one-electron and two-electrons integrals in Equation 2.18 are given by 91$]$

$$
\begin{aligned}
h_{i} & =\int \phi_{i}^{*}(\mathbf{x})\left[-\frac{1}{2} \nabla^{2}+V_{e x t}(\mathbf{r})\right] \phi_{i}(\mathbf{x}) d^{3} \mathbf{x} \\
J_{i j} & =\iint \frac{\left|\phi_{i}(\mathbf{x})\right|^{2}\left|\phi_{j}\left(\mathbf{x}^{\prime}\right)\right|^{2} d^{3} \mathbf{x} d^{3} \mathbf{x}^{\prime}}{\left|\mathbf{r}-\mathbf{r}^{\prime}\right|} \\
K_{i j} & =\iint \frac{\phi_{j}^{*}\left(\mathbf{x}^{\prime}\right) \phi_{i}^{*}(\mathbf{x}) \phi_{i}\left(\mathbf{x}^{\prime}\right) \phi_{j}(\mathbf{x}) d^{3} \mathbf{x} d^{3} \mathbf{x}^{\prime}}{\left|\mathbf{r}-\mathbf{r}^{\prime}\right|}
\end{aligned}
$$

It may be noted that $\phi_{i}(\mathbf{x})$ are composite spin orbitals that contains both the spin and space parts 91]; these space and spin parts are detachable in the absence of magnetic interactions in the many-body Hamiltonian. Furthermore, the integration over the space coordinates and summation over the spin coordinates are denoted by a single integration over the composite variable $\mathbf{x}$ in Equations 2.19] 91$]$.

$$
\begin{aligned}
& \phi_{i}(\mathbf{x})=\phi_{i}(\mathbf{r})\{\alpha \quad \text { or } \beta \\
& \sum_{\text {spin }} \int d^{3} \mathbf{r} \equiv \int d^{3} \mathbf{x}
\end{aligned}
$$


Here $\alpha$ and $\beta$ are the two possible spin states. In Equations 2.19 and 2.20, $\mathbf{x}$ is the four dimensional variable of the spin and space coordinates [91]. These spin orbitals satisfy the orthonormality condition $\int \phi_{i}^{\star}(\mathbf{x}) \phi_{j}(\mathbf{x}) d^{3} \mathbf{x}=\delta_{i j}$. The integrals $J_{i j}$ and $K_{i j}$ are the Coulomb and Exchange integrals. $J_{i j}$ has a purely classical origin and is due to the classical Coulomb interaction between two overlapping charge distributions. It may be noted that this term was present in the Hartree theory. $K_{i j}$, on the other hand, has a purely quantum origin and arises because the total wave function of electrons should be anti-symmetric upon the exchange of two electrons. If two electrons have their spins pointing in the same direction, the total energy in Equation 2.18 decreases as $K_{i j}>0$. This suggests the exchange interactions tend to keep electrons of the same spins away from each other by lowering their repulsive energy [91]. Such reduction in the energy of electrons arising due to the anti-symmetry of their total wave function is called exchange energy [87]. In this sense, there is some sort of correlation between particles of the same spins in Hartree-Fock theory. However, this theory does not treat properly the correlation between electrons of opposite spins. If two electrons have their spins pointing in the opposite direction, $K_{i j}=0$. Therefore, the energy of electrons in the triplet state is lower energy than the energy when they are in a singlet state. Furthermore, if $\mathrm{i}=\mathrm{j}, J_{i j}=K_{i j}$. This means there is no self-interaction error in Hartree-Fock theory and this is the reason why a part of Hartree-Fock exchange is included in the hybrid functionals in DFT to partially correct the self-interaction error. 
Next, we minimize the expression of energy in Equation 2.18 using Lagrange's method of undetermined multiplier, $\lambda_{i j}$, under the constraint that these spin orbitals have to be orthonormal. For this we define a functional $F\left(\phi_{i}, \phi_{i}^{*}\right)$ as 91$]$

$$
\begin{aligned}
F\left(\phi_{i}, \phi_{i}^{*}\right) & =\sum_{i=1}^{N_{e}} \int \phi_{i}^{*}(\mathbf{x})\left[-\frac{1}{2} \nabla^{2}+V_{e x t}(\mathbf{r})\right] \phi_{i}(\mathbf{x}) d^{3} \mathbf{x} \\
& +\frac{1}{2} \sum_{i=1}^{N_{e}} \sum_{j=i}^{N_{e}} \iint \frac{\left|\phi_{i}(\mathbf{x})\right|^{2}\left|\phi_{j}\left(\mathbf{x}^{\prime}\right)\right|^{2} d^{3} \mathbf{x} d^{3} \mathbf{x}^{\prime}}{\left|\mathbf{r}-\mathbf{r}^{\prime}\right|} \\
& -\frac{1}{2} \sum_{i=1}^{N_{e}} \sum_{j=i}^{N_{e}} \iint \frac{\phi_{j}^{*}\left(\mathbf{x}^{\prime}\right) \phi_{i}^{*}(\mathbf{x}) \phi_{i}\left(\mathbf{x}^{\prime}\right) \phi_{j}(\mathbf{x}) d^{3} \mathbf{x} d^{3} \mathbf{x}^{\prime}}{\left|\mathbf{r}-\mathbf{r}^{\prime}\right|} \\
& -\sum_{i j} \lambda_{j i}\left(\left\langle\phi_{i} \mid \phi_{j}\right\rangle-\delta_{i j}\right)
\end{aligned}
$$

Using minimization condition $\frac{\delta F\left(\phi_{i}, \phi_{i}^{*}\right)}{\delta \phi_{k}^{*}}=0$, we get [91]

$$
\left[-\frac{1}{2} \nabla^{2}+V_{e x t}+V_{H}+V_{x}\right] \phi_{i}(\mathbf{x})=\sum_{j=1}^{N_{e}} \phi_{j}(\mathbf{x}) \lambda_{j i}
$$

The matrix of Lagrange undetermined multiplier can be diagonalized by using unitary transformation to get a set of single-electron Schrödinger wave-like equations called Hartree-Fock equations as 91]

$$
\left[-\frac{1}{2} \nabla^{2}+V_{e x t}+V_{H}+V_{x}\right] \phi_{i}(\mathbf{x})=\epsilon_{i} \phi_{i}(\mathbf{x})
$$

In Equation 2.23,

$$
V_{e x t}=-\sum_{I=1}^{N_{I}} \frac{Z_{I}}{\left|\mathbf{r}-\mathbf{R}_{I}\right|}
$$




$$
\begin{gathered}
V_{H}=\sum_{j=1}^{N_{e}} \int \frac{\left|\phi_{j}\left(\mathbf{x}^{\prime}\right)\right|^{2} d^{3} \mathbf{x}^{\prime}}{\left|\mathbf{r}-\mathbf{r}^{\prime}\right|} \\
V_{x}=-\left[\sum_{j=1}^{N_{e}} \int \frac{\phi_{j}^{*}\left(\mathbf{x}^{\prime}\right) \phi_{i}\left(\mathbf{x}^{\prime}\right) d^{3} \mathbf{x}^{\prime}}{\left|\mathbf{r}-\mathbf{r}^{\prime}\right|}\right] \frac{\phi_{j}(\mathbf{x})}{\phi_{i}(\mathbf{x})}
\end{gathered}
$$

Here, $V_{H}$ and $V_{x}$ are the Hartree and exchange term, respectively. In short [91,

$$
H_{i}^{e f f} \phi_{i}(\mathbf{x})=\epsilon_{i} \phi_{i}(\mathbf{x})
$$

Here, $\mathrm{i}=1,2, \ldots \ldots, N_{e}$. Thus, a single equation for $N_{e}$ electrons has been reduced to $N_{e}$ effective single-electron equations using Hartree Fock theory. These equations are still coupled as the solution of the one-electron problem enters the differential equation for the second through the effective potential[91]. This makes these equations nonlinear and therefore, there are no exact solutions to these equations. These equations have to be solved self-consistently/iteratively in computers.

\subsection{Correlation Energy}

In Hartree-Fock theory, the electrons interact with an average potential arising due to all the other electrons and the nuclei. Therefore, it neglects the interaction between the electrons due to their instantaneous positions and tends to keep them away 
from each other's vicinity. Such interactions between the electrons due to their instantaneous positions are called dynamic correlation. In Hartree-Fock theory, the many-electron wave function is represented by a single Slater determinant that may not represent the true wave function of a many-electron system. In reality, the true wave function should be the linear combination of all the possible Slater determinants corresponding to the single, double and higher-order electronic excitations. The other form of correlation, also called static correlation, is related to the existence of the several nearly degenerate configurations (Slater determinants) that have not been taken into account when the total wave function of electrons is approximated by a single Slater determinant. Since the wave function of electrons is not exact, the energy we get in Hartree-Fock theory is always larger than the true energy of the system. The correlation energy, defined as the difference between the exact non-relativistic energy and the Hartree Fock theory, can be expressed mathematically as 98]

$$
E_{\text {corr }}=E_{\text {exact }}-E_{H F}
$$

Correlation energy is negative and therefore it lowers the energy of the system. This energy is missing in Hartree-Fock theory. Both dynamic and static correlation can be treated properly by taking a linear combination of many possible Slater determinants as in configurational interaction (CI). However, full CI can be computationally inefficient even for systems with a few atoms and DFT becomes an obvious choice as it allows us to simulate much larger systems that full CI can't. 


\subsection{Density Functional Theory (DFT)}

In DFT, it is the ground-state electron density $n(\mathbf{r})$ that determines all the ground state properties of the system including the ground state wave function. The electron density $n(\mathbf{r})$ has the following properties:

$$
\begin{aligned}
& n(\mathbf{r}) \geq 0 \\
& n(\mathbf{r})=N_{e} \int \ldots \int\left|\Phi\left(\mathbf{r}, \mathbf{r}_{2}, \mathbf{r}_{3}, \ldots, \mathbf{r}_{N_{e}}\right)\right|^{2} d^{3} \mathbf{r}_{2} d^{3} \mathbf{r}_{3} \ldots d^{3} \mathbf{r}_{N_{e}}
\end{aligned}
$$

Integration of density over entire volume gives the total number of electrons $N_{e}$ i.e.

$$
\int n(\mathbf{r}) d^{3} \mathbf{r}=N_{e}
$$

$$
n(\mathbf{r})=\sum_{i=1}^{N_{e}} f_{i}\left|\phi_{i}(\mathbf{r})\right|^{2}
$$

$f_{i}$ is the occupation probability. It may be noted that only the occupied orbitals contribute to the electron density.

\subsubsection{Why DFT?}

The main advantage of DFT over the WFT based approach is that it makes the computation of many-electron systems possible in computers. To explain this, 
let's consider a many-electron system with $N_{e}$ electrons. The wave function, $\Phi\left(r_{1}, r_{2}, r_{3}, \ldots, r_{N_{e}}\right)$, is a function of $3 N_{e}$ variables. Let's say we want to represent this wave function using $M$ grid points in space. Then at each grid point, we have $3 N_{e}$ variables so that the total numbers of variables in space becomes $M^{3 N_{e}}$. In the simplest case, when we have $N_{e}=10$ electrons and $\mathrm{M}=100$ grid points, the total number of variables become $10^{60}$. Considering that each variable can be stored using 10 bytes of memory, we need around $10^{52}$ GB to store the wave function of a many-electron system which is almost impossible. But if we use DFT to represent an electron density using $\mathrm{M}=100$ grid points, we have only $10^{6}$ variables as density is a function of 3 variables. If we compute the memory required in computers to store the density, we need only $10 \mathrm{MB}$ of space. In a Kohn-Sham DFT, an electron density is constructed from the $N_{e}$ occupied orbitals using $n(\mathbf{r})=\sum_{i=1}^{N_{e}} f_{i}\left|\phi_{i}(\mathbf{r})\right|^{2}$. So, we need $N_{e} \times 100^{3}$ variables to represent a system of $N_{e}$ electrons. For $N_{e}=10$, we need only 100 MB of memory to store all these orbitals. Therefore, DFT makes simulation of much larger electronic systems possible in computers.

DFT is based on Hohenberg-Kohn theorems I and II for an inhomogenous electron gas 99]. Before discussing them, let's discuss the Thomas-Fermi model for a homogeneous system of electron gas which laid the foundation for these theorems. 


\subsubsection{Thomas-Fermi Model}

It was Thomas-Fermi who in 1927 expressed the kinetic energy of non-interacting homogenous electron gas in terms of the local electron density at a point $\mathbf{r}$. Dirac, in 1930, extended this model by including the exchange energy that is still used as an approximation to the exchange term in Local Density Approximation (LDA) in modern DFT[98]. To obtain the expression of kinetic energy as a function of density, we discretize the space into cubic cells. Each of them have a volume $\Delta V=l^{3}$, where l is the length of a side of the cube. Let $\Delta N$ be the number of electrons in $\Delta V$. These $\Delta V$ 's are independent of each other and may have different number of electrons. Consequently, the total kinetic energy of electrons in a cell can be expressed as 98

$$
\begin{aligned}
\Delta E & =\frac{3}{5} \Delta N \epsilon_{F} \\
& =\frac{3 h^{2}}{10 m}\left(\frac{3}{8 \pi}\right)^{2 / 3} l^{3}\left(\frac{\Delta N}{l^{3}}\right)^{5 / 3}
\end{aligned}
$$

The term $n=\frac{\Delta N}{\Delta V}$ is called the density of electrons for each cubic cell and might be different for different cells. In the limit $\Delta V \rightarrow 0, n=\frac{\Delta N}{l^{3}}=n(\mathbf{r})$. Subsequently, the summation over all cells in the space can be changed to integration while calculating the total kinetic energy of all the electrons in space. The Thomas-Fermi total kinetic 
energy functional in atomic units is therefore 98

$$
T_{T F}[n(\mathbf{r})]=\frac{3}{10}\left(3 \pi^{2}\right)^{2 / 3} \int[n(\mathbf{r})]^{5 / 3} d^{3} \mathbf{r}
$$

The Thomas-Fermi total energy functional can be expressed as 98

$$
\begin{aligned}
E_{T F}[n(\mathbf{r})] & =\frac{3}{10}\left(3 \pi^{2}\right)^{2 / 3} \int[n(\mathbf{r})]^{5 / 3} d^{3} \mathbf{r}-Z \int \frac{n(\mathbf{r})}{r} d^{3} \mathbf{r} \\
& +\frac{1}{2} \iint \frac{n(\mathbf{r}) n\left(\mathbf{r}^{\prime}\right)}{\left|\mathbf{r}-\mathbf{r}^{\prime}\right|} d^{3} \mathbf{r} d^{3} \mathbf{r}^{\prime}
\end{aligned}
$$

In Equation 2.31, the first term is the Thomas-Fermi total kinetic energy functional. The second term represents the potential energy of attraction between the electrons and the ions. Similarly, the third term is the potential energy of repulsion between the electrons. It may be noted that in writing Equation 2.31, Thomas-Fermi neglected the exchange-correlation energy. The energy functional in Equation 2.31 can be minimized under the constraint $\int n(\mathbf{r}) d^{3}(\mathbf{r})=N_{e}$ to get the Euler-Lagrange equation 98

$$
\begin{aligned}
\mu_{F T} & =\frac{\delta E_{F T}[n(\mathbf{r})]}{\delta n(\mathbf{r})} \\
& =\frac{1}{2}\left(3 \pi^{2}\right)^{2 / 3}[n(\mathbf{r})]^{2 / 3}-\frac{Z}{r}+\int \frac{n\left(\mathbf{r}^{\prime}\right)}{\left|\mathbf{r}-\mathbf{r}^{\prime}\right|} d^{3} \mathbf{r}^{\prime} \\
& =\frac{1}{2}\left(3 \pi^{2}\right)^{2 / 3}[n(\mathbf{r})]^{2 / 3}-\phi(\mathbf{r})
\end{aligned}
$$

In Equation 2.32, $\phi(\mathbf{r})$ is the potential due to the nuclei and electrons at a position $\mathbf{r}[98$. Equation 2.31 tells that the total energy of the system can be calculated once we know the density. This idea of expressing the energy in terms of the electron 
density was used by Hohenberg-Kohn to propose two theorems on which the modern DFT is based. It may be noted that the Thomas-Fermi model is the simplest model for the total energy of a system of electrons and is an approximation to DFT.

\subsubsection{The Hohenberg-Kohn Theorem I}

The Hohenberg-Kohn Theorem I states that the external potential $V_{\text {ext }}(\mathbf{r})$ is a unique functional of the ground state electron density $n(\mathbf{r})$ within a constant [98, 99]. So the basic variable in DFT is $n(\mathbf{r})$ 99. As the many-body Hamiltonian is determined by the external potential $V_{\text {ext }}(\mathbf{r})$, the full many-body ground state problem is also a unique functional of $n(\mathbf{r})$ [99].

To prove this theorem, we consider two systems such that $V_{\text {ext }}(\mathbf{r}) \neq V_{\text {ext }}^{\prime}(\mathbf{r})+C$. Both of these systems have the same ground state density i.e $n(\mathbf{r})=n\left(\mathbf{r}^{\prime}\right)$. Let their respective Hamiltonians be $H$ and $H^{\prime}$.

System I: $\quad V_{\text {ext }}(\mathbf{r}), \quad \hat{H}, \quad n(\mathbf{r}), \quad \Phi(\mathbf{r}), \quad E, \quad N_{e}$

SystemII: $\quad V_{e x t}^{\prime}(\mathbf{r}), \quad \hat{H}^{\prime}, \quad n(\mathbf{r}), \quad \Phi^{\prime}(\mathbf{r}), \quad E^{\prime}, \quad N_{e}$ 
The ground state energy $E_{0}$ for the System I can be expressed as 98

$$
\begin{aligned}
E_{0}= & \langle\Phi|\hat{H}| \Phi\rangle \\
& <\left\langle\Phi^{\prime}|\hat{H}| \Phi^{\prime}\right\rangle \\
& =\left\langle\Phi^{\prime}\left|\hat{H}^{\prime}\right| \Phi^{\prime}\right\rangle+\left\langle\Phi^{\prime}\left|\hat{H}-\hat{H}^{\prime}\right| \Phi^{\prime}\right\rangle \\
& =\left\langle\Phi^{\prime}\left|\hat{H}^{\prime}\right| \Phi^{\prime}\right\rangle+\int\left[V_{\text {ext }}(\mathbf{r})-V_{\text {ext }}^{\prime}(\mathbf{r})\right] n(\mathbf{r}) d^{3} \mathbf{r} \\
& <E_{0}^{\prime}+\int\left[V_{\text {ext }}(\mathbf{r})-V_{\text {ext }}^{\prime}(\mathbf{r})\right] n(\mathbf{r}) d^{3} \mathbf{r}
\end{aligned}
$$

Similarly, the ground state energy for the System II can be expressed as 98

$$
E_{0}^{\prime}<E_{0}+\int\left[V_{e x t}^{\prime}(\mathbf{r})-V_{e x t}(\mathbf{r})\right] n(\mathbf{r}) d^{3} \mathbf{r}
$$

Adding Equations 2.33 and 2.34 ,

$$
E_{0}+E_{0}^{\prime}<E_{0}^{\prime}+E_{0}
$$

Thus we arrive at a contradiction. This means there is no existence of two external potentials that differ by more than a constant and give same ground state electron density $n(\mathbf{r})$. The electronic Hamiltonian is

$$
\hat{H}=\hat{T}_{e}+\hat{V}_{e e}+\hat{V}_{e N}
$$

In DFT, it is the electron density that determines the total number of electrons 
$N_{e}$, the external potential $V_{e x t}$, and all the ground state properties like energy, wave function, force etc. Therefore, the total energy is the functional of the ground state density $n(\mathbf{r})$ and can be expressed as [98, 99]

$$
\begin{aligned}
E_{v}[n] & =\langle\Phi|\hat{H}| \Phi\rangle \\
& =\left\langle\Phi\left|\hat{T}_{e}\right| \Phi\right\rangle+\left\langle\Phi\left|\hat{V}_{e e}\right| \Phi\right\rangle+\left\langle\Phi\left|\hat{V}_{e N}\right| \Phi\right\rangle \\
& =T[n]+V_{e e}[n]+V_{e N}[n] \\
& =F_{H K}[n]+V_{e N}[n] \\
& =F_{H K}[n]+\int V_{e x t}(\mathbf{r}) n(\mathbf{r}) d^{3} \mathbf{r}
\end{aligned}
$$

where

$$
\begin{gathered}
F_{H K}[n]=T[n]+V_{e e}[n] \\
V_{e e}[n]=J[n]+\text { non - classical term }
\end{gathered}
$$

In Equation 2.37, $\Phi$ is the ground state wave function that corresponds to the electron density $n(\mathbf{r})$. The subscript $v$ in $E_{v}$ means that the energy depends explicitly on the external potential $V_{\text {ext }}$ [98]. $J[n(\mathbf{r})]$, in Equation 2.38 , is the Coulomb repulsion between the electron distribution and has classical origin as explained earlier. The non-classical term is not known in DFT and arises due to exchange-correlation between electrons in materials. It's origin is quantum in nature. $F_{H K}[n(\mathbf{r})]$, in Equation 2.38, is a universal functional of the electron density and is valid for any external 
potential and the number of electrons in the system.

\subsubsection{The Hohenberg-Kohn Theorem II}

The Hohenberg-Kohn Theorem II is basically a variational principle and states that for any trial density $n^{\prime}(\mathbf{r})$ such that $n^{\prime}(\mathbf{r}) \geq 0$ and $\int n^{\prime}(\mathbf{r}) d^{3} \mathbf{r}=N_{e}$, the energy obtained satisfies the following condition 98

$$
E_{v}\left[n_{0}\right] \leq E_{v}\left[n^{\prime}\right]
$$

$E_{v}\left[n_{0}\right]$ is the ground state energy corresponding to the ground state electron density $n_{0}(\mathbf{r})$ and external potential $V_{\text {ext }}(\mathbf{r}) ; \Phi_{0}$ is the ground state wave function. To prove this theorem, let us suppose that $V_{\text {ext }}^{\prime}(\mathbf{r})$ and $\Phi^{\prime}$ be the external potential and the wave function determined by the electron density $n^{\prime}(\mathbf{r})$. This means if we consider a problem with $V_{\text {ext }}(\mathbf{r})$ as the external potential, $\Phi^{\prime}$ becomes the trial wave function and therefore, we can write 98

$$
\begin{aligned}
E_{v}\left[n_{0}\right]=\left\langle\Phi_{0}|\hat{H}| \Phi_{0}\right\rangle & \leq\left\langle\Phi^{\prime}|\hat{H}| \Phi^{\prime}\right\rangle \\
& =F_{H K}\left[n^{\prime}\right]+\int n^{\prime}(\mathbf{r}) V_{\text {ext }}(\mathbf{r}) d^{3} \mathbf{r} \\
& =E_{v}\left[n^{\prime}\right] \\
\Rightarrow E_{v}\left[n_{0}\right] \leq E_{v}\left[n^{\prime}\right] &
\end{aligned}
$$




\subsubsection{Levy Constrained Search}

There are two limitations of Hohenberg-Kohn theorems: (i) it assumes that there should not be any degeneracy in the ground state (ii) the electron density has to be v-representable. However, the general condition for having a v-representable density is not known making practical implementation of the Hohenberg-Kohn theorem difficult. Levy constrained approach formulation[100, 101] gets rid of both of these limitations in the original Hohenberg-Kohn theorem by converting the v-representability problem to N-representability. Let's discuss it. Let's consider a ground state density $n_{0}(\mathbf{r})$ that

can be constructed from many different wave functions. Let $\Phi_{n_{0}}$ and $\Phi_{0}$ be any two of them such that $\Phi_{0}$ is the true ground state wave function. Using variational principle, we can write 98

$$
\begin{aligned}
& \left\langle\Phi_{n_{0}}|\hat{H}| \Phi_{n_{0}}\right\rangle \geq\left\langle\Phi_{0}|\hat{H}| \Phi_{0}\right\rangle \\
& \Rightarrow\left\langle\Phi_{n_{0}}\left|\hat{T}+\hat{V}_{e e}\right| \Phi_{n_{0}}\right\rangle+\int n_{0}(\mathbf{r}) V_{\text {ext }}(\mathbf{r}) d^{3} \mathbf{r} \geq\left\langle\Phi_{0}\left|\hat{T}+\hat{V}_{e e}\right| \Phi_{0}\right\rangle+\int n_{0}(\mathbf{r}) V_{\text {ext }}(\mathbf{r}) d^{3} \mathbf{r} \\
& \Rightarrow\left\langle\Phi_{n_{0}}\left|\hat{T}+\hat{V}_{e e}\right| \Phi_{n_{0}}\right\rangle \geq\left\langle\Phi_{0}\left|\hat{T}+\hat{V}_{e e}\right| \Phi_{0}\right\rangle
\end{aligned}
$$

This means although a given ground state density $n_{0}(\mathbf{r})$ can be obtained via integration from many different wave functions $\left(\Phi_{n_{0}}\right.$ and $\left.\Phi_{0}\right)$, it is only the ground state 
wave function $\Phi_{0}$ that minimizes $T+V_{e e}$. The right hand side of Equation 2.41 is 98$]$

$$
\begin{aligned}
F_{H K}\left[n_{0}\right] & =\left\langle\Phi_{0}\left|\hat{T}+\hat{V}_{e e}\right| \Phi_{0}\right\rangle \\
& =\min _{\mid \Phi>\rightarrow n_{0}}\left\langle\Phi\left|\hat{T}+\hat{V}_{e e}\right| \Phi\right\rangle
\end{aligned}
$$

This is Levy's constrained search approach. As stated earlier, it removes the limitation of the Hohenberg-Kohn theorem that there should not be degeneracy in the ground state and allows us to generalize the definition of $F_{H K}\left[n_{0}\right]$ from v-representable density to N-representable density as 98

$$
F[n]=\min _{\mid \Phi>\rightarrow n}\left\langle\Phi\left|\hat{T}+\hat{V}_{e e}\right| \Phi\right\rangle
$$

Using Equations 2.42 and 2.43 , we can write 98

$$
F_{H K}\left[n_{0}\right]=F\left[n_{0}\right]
$$

for any v-representable density $n_{0}$. The ground state energy can be written as 98]

$$
E_{0}[n]=\min _{n}\left[\min _{\mid \Phi>\rightarrow n}\left\langle\Phi\left|\hat{T}+\hat{V}_{e e}\right| \Phi\right\rangle+\int V_{e x t}(\mathbf{r}) n(\mathbf{r}) d^{3} \mathbf{r}\right]
$$

There are two minimization constraints in Equation 2.45; the inner minimization is over all the wave functions that integrate to give the same density $n(\mathbf{r})$ and the outer is over all the densities that integrate to give total number of electrons $N_{e}[98$. 
Equation 2.45 can be written as 98

$$
\begin{aligned}
E_{0}[n] & =\min _{n}\left[F[n]+\int V_{e x t}(\mathbf{r}) n(\mathbf{r}) d^{3} \mathbf{r}\right] \\
& =\min _{n} E[n]
\end{aligned}
$$

Thus we converted the v-representability problem as proposed originally by Hohenberg-Kohn to an N-representable problem using the idea of Levy's constrained search approach.

\subsubsection{The Kohn-Sham Formulation}

Hohenberg-Kohn theorem does not give us an idea about the nature of the universal functional $F[n(\mathbf{r})]$, neither it provides a way to obtain the ground-state (GS) properties of the system from the ground-state electron density. Therefore, we need Kohn-Sham formulation[102]. To derive the Kohn-Sham equations, we consider two systems as illustrated in Figure 2.2. One of them is a real system of interacting electrons that includes the exchange-correlation between them. The other is an auxiliary system of non-interacting electrons in which the electrons move in an effective potential called Kohn-Sham potential. We further assume that both of these systems has the same electron density $n(\mathbf{r})$. 
Interacting particles

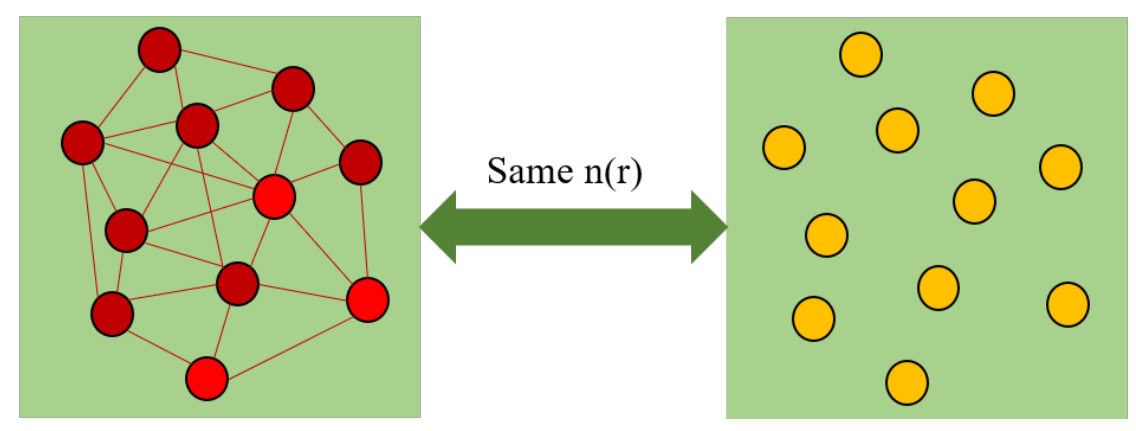

Figure 2.2: One to one mapping of the real interacting system with a non-interacting system. Both systems have the same ground state density $n(\mathbf{r})$.

The energy functional for the electrons moving in an effective Kohn-Sham potential $V^{K S}(\mathbf{r})$ is 98

$$
E[n]=T_{s}[n]+\int V^{K S}(\mathbf{r}) n(\mathbf{r}) d^{3} \mathbf{r}
$$

$T_{s}[n(\mathbf{r})]$ is the total kinetic energy functional of the auxiliary system of noninteracting electrons and can be expressed as sum of the kinetic energies of the non-interacting single particle orbitals $\phi_{i}$ 's as 98

$$
\begin{aligned}
T_{s}[n] & =\left\langle\Phi\left|-\frac{1}{2} \nabla^{2}\right| \Phi\right\rangle \\
& =-\frac{1}{2} \sum_{i=1}^{N_{e}} \int \phi_{i}^{*}(\mathbf{r}) \nabla^{2} \phi_{i}(\mathbf{r}) d^{3} \mathbf{r}
\end{aligned}
$$

where $\Phi$ is the Slater determinant. The energy functional in Equation 2.47 can 
be minimized by using the Lagrange's undermined multiplier under the constraint $\int n(\mathbf{r}) d^{3} \mathbf{r}=N_{e}$. To do so, we write the energy functional as 98]

$$
E[n]=T_{s}[n]+\int V^{K S}(\mathbf{r}) n(\mathbf{r}) d^{3} \mathbf{r}-\mu\left[\int n(\mathbf{r}) d^{3} \mathbf{r}-N_{e}\right]
$$

Using the minimization condition $\frac{\delta E[n]}{\delta n(\mathbf{r})}=0$, we get the Euler-Lagrange equation for a system of non-interacting electrons 98,103 ,

$$
\mu=\frac{\delta T_{s}[n]}{\delta n(\mathbf{r})}+V^{K S}(\mathbf{r})
$$

For a system of interacting electrons, the energy functional can be expressed in terms of the kinetic energy functional of the non-interacting electrons as 98

$$
E[n]=T_{s}[n]+\frac{1}{2} \iint \frac{n(\mathbf{r}) n\left(\mathbf{r}^{\prime}\right)}{\left|\mathbf{r}-\mathbf{r}^{\prime}\right|} d^{3} \mathbf{r} d^{3} \mathbf{r}^{\prime}+\int V_{e x t}(\mathbf{r}) n(\mathbf{r}) d^{3} \mathbf{r}+E_{x c}[n]
$$

where $E_{x c}$ is the exchange correlation energy functional (to be discussed later) and is given by 98

$$
E_{x c}=T[n]-T_{s}[n]+E_{e e}[n]-E_{H}[n]
$$

In Equation $2.52, T[n(\mathbf{r})]$ and $E_{e e}[n(\mathbf{r})]$ are the exact kinetic and electron-electron interaction energies of the interacting electrons respectively. Let's minimize the energy 
functional in Equation 2.51 using the Lagrange's undermined multiplier under the constraint, $\int n(\mathbf{r}) d^{3} \mathbf{r}=N_{e}$. For this we rewrite the energy functional for interacting electrons as 98

$$
\begin{aligned}
E[n] & =T_{s}[n]+\frac{1}{2} \iint \frac{n(\mathbf{r}) n\left(\mathbf{r}^{\prime}\right)}{\left|\mathbf{r}-\mathbf{r}^{\prime}\right|} d^{3} \mathbf{r} d^{3} \mathbf{r}^{\prime} \\
& +\int V_{e x t}(\mathbf{r}) n(\mathbf{r}) d^{3} \mathbf{r}+E_{x c}[n]-\mu^{\prime}\left[\int n(\mathbf{r}) d^{3} \mathbf{r}-N_{e}\right]
\end{aligned}
$$

Minimization of Equation 2.53 will give Euler-Lagrange equation for interacting electrons [98, 103 ]

$$
\mu^{\prime}=\frac{\delta T_{s}[n]}{\delta n(\mathbf{r})}+\int \frac{n\left(\mathbf{r}^{\prime}\right)}{\left|\mathbf{r}-\mathbf{r}^{\prime}\right|} d^{3} \mathbf{r}^{\prime}+V_{e x t}(\mathbf{r})+\frac{\delta E_{x c}[n]}{\delta n(\mathbf{r})}
$$

Comparing Equations 2.50 and 2.54, the effective potential in which the electrons move is given by 98,103$]$

$$
\begin{aligned}
V^{K S}(\mathbf{r}) & =\int \frac{n\left(\mathbf{r}^{\prime}\right)}{\left|\mathbf{r}-\mathbf{r}^{\prime}\right|} d^{3} \mathbf{r}^{\prime}+V_{e x t}(\mathbf{r})+\frac{\delta E_{x c}[n]}{\delta n(\mathbf{r})} \\
& =V_{H}(\mathbf{r})+V_{e x t}(\mathbf{r})+V_{x c}(\mathbf{r})
\end{aligned}
$$

Both the minimizations (one for the auxiliary system and other for the interacting system) have the identical solution for the density if the effective potential is chosen to be the sum of the three terms $\left(V_{H}(\mathbf{r}), V_{\text {ext }}(\mathbf{r})\right.$ and $\left.V_{x c}(\mathbf{r})\right)$ as in Equation 2.55]103]. This means that the density of the interacting system in an external potential $V_{\text {ext }}(\mathbf{r})$ can be 
obtained by solving the equations of the non-interacting electrons in the effective potential $V^{K S}(\mathbf{r})$ [103]. In Equation 2.55, $V_{x c}(\mathbf{r})$ is the exchange-correlation potential. To get single electron Kohn-Sham equations, we minimize Equation 2.51 under the constraint that the electrons orbitals have to be orthonormal i.e. $\int \phi_{i}(\mathbf{r}) \phi_{j}(\mathbf{r}) d^{3} \mathbf{r}=\delta_{i j}$. For this we rewrite energy functional as 98

$$
\begin{aligned}
E\left(\phi_{i}^{*}, \phi_{i}\right) & =-\frac{1}{2} \sum_{i=1}^{N_{e}} \int \phi_{i}^{*}(\mathbf{r}) \nabla^{2} \phi_{i}(\mathbf{r}) d^{3} \mathbf{r}+\frac{1}{2} \iint \frac{n(\mathbf{r}) n\left(\mathbf{r}^{\prime}\right)}{\left|\mathbf{r}-\mathbf{r}^{\prime}\right|} d^{3} \mathbf{r} d^{3} \mathbf{r}^{\prime} \\
& +\int V_{e x t}(\mathbf{r}) n(\mathbf{r}) d^{3} \mathbf{r}+E_{x c}[n]-\sum_{i j} \epsilon_{i j}\left[\int \phi_{i}^{*}(\mathbf{r}) \phi_{j}(\mathbf{r}) d^{3} \mathbf{r}-\delta_{i j}\right]
\end{aligned}
$$

Using the minimization condition $\frac{\delta E\left(\phi_{i}^{*}, \phi_{i}\right)}{\delta \phi_{k}^{*}}=0$ and then diagonalizing the Lagrange's undetermined multiplier $\epsilon_{i j}$ using unitary transformation, we get single particle equations of the form 98

$$
\begin{aligned}
{\left[-\frac{1}{2} \nabla^{2}+V_{e x t}(\mathbf{r})+\int \frac{n\left(\mathbf{r}^{\prime}\right)}{\left|\mathbf{r}-\mathbf{r}^{\prime}\right|} d^{3} \mathbf{r}^{\prime}+V_{x c}(\mathbf{r})\right] \phi_{i}(\mathbf{r}) } & =\epsilon_{i} \phi_{i}(\mathbf{r}) \\
& {\left[-\frac{1}{2} \nabla^{2}+V^{K S}(\mathbf{r})\right] \phi_{i}(\mathbf{r})=\epsilon_{i} \phi_{i}(\mathbf{r}) }
\end{aligned}
$$

where,

$$
V^{K S}(\mathbf{r})=\int \frac{n\left(\mathbf{r}^{\prime}\right)}{\left|\mathbf{r}-\mathbf{r}^{\prime}\right|} d^{3} \mathbf{r}^{\prime}+V_{e x t}(\mathbf{r})+V_{x c}(\mathbf{r})
$$

In Equation 2.57, $i=1,2,3, \ldots \ldots N_{e}$. Thus, Kohn-Sham formulation splits a single problem of $N_{e}$ electrons to $N_{e}$ single electron problems with the electrons moving 
in an effective Kohn-Sham potential $V^{K S}(\mathbf{r})$. The set of the Kohn-Sham equations in Equation 2.57 are non-linear equations. These have to be solved self-consistently (iteratively) starting from some guess for the electron density to get well conserved density that can be constructed from the occupied orbitals using 98

$$
n(\mathbf{r})=\sum_{i=1}^{N_{e}} \phi_{i}^{*}(\mathbf{r}) \phi_{i}(\mathbf{r})
$$

Such iterative method will give a self-consistent ground state density. The total energy in Kohm-Sham theory is then calculated from the self-consistent density by using the relation 98 , 103 ]

$$
E=\sum_{i=1}^{N_{e}} \epsilon_{i}-\frac{1}{2} \iint \frac{n(\mathbf{r}) n\left(\mathbf{r}^{\prime}\right)}{\left|\mathbf{r}-\mathbf{r}^{\prime}\right|} d^{3} \mathbf{r} d^{3} \mathbf{r}^{\prime}-\int V_{x c}(\mathbf{r}) n(\mathbf{r}) d^{3} \mathbf{r}+E_{x c}[n]
$$

It may be noted that Kohn-Sham's theory is a single orbital theory. $\epsilon_{i}$, in Equation 2.59 , is the energy corresponding to the orbital $\phi_{i}$.

\subsubsection{Exchange-Correlation Energy}

In writing the energy functional (Equation 2.51) for interacting electrons, we expressed it in terms of the kinetic energy functional $\left(T_{s}[n]\right)$ for the non-interacting 
electrons. The Kohn-Sham kinetic energy functional $\left(T_{s}[n]\right)$ is not the true representative of the kinetic energy of real interacting electrons. Further, the exact form of the electron interaction energy functional $E_{e e}[n]$ is not known. All the unknown quantities in DFT are contained in the exchange-correlation energy functional and is the difference in exact kinetic energy $(T[n])$ and Kohn-Sham kinetic energy $\left(T_{s}[n]\right)$, plus the difference in exact electron-electron interaction energy $\left(E_{e e}[n]\right)$ and the Hartree energy $\left(E_{H}[n]\right)$. Quantitatively, it can be expressed as 98

$$
E_{x c}=T[n]-T_{S}[n]+E_{e e}[n]-E_{H}[n]
$$

Since the exact form of the exchange-correlation energy functional is not known, we need to make approximations for it. The accuracy of the result in DFT depends on how well the approximations for the exchange-correlation energy are made. Some of the approximations like LDA, GGA, etc will be discussed in the next subsections.

\subsubsection{Local Density Approximation}

The simplest approximation for the exchange-correlation energy functional is the Local Density Approximation (LDA) in which it is assumed that the exchangecorrelation energy at a point $\mathbf{r}$ is the same as that of a locally uniform electron 
gas. In this approximation, the exchange-correlation energy is given by [87, 98]

$$
E_{x c}^{L D A}[n]=\int \epsilon_{x c}(n) n(\mathbf{r}) d^{3} \mathbf{r}
$$

where $\epsilon_{x c}(n)=\epsilon_{x c}^{h o m}(n)$ is the exchange-correlation energy per particle of a uniform electron gas having density $n$. The exchange-correlation potential $V_{x c}^{L D A}$ is 98$]$

$$
V_{x c}^{L D A}(\mathbf{r})=\frac{\delta E_{x c}^{L D A}[n]}{\delta n(\mathbf{r})}=\frac{\partial\left[n(\mathbf{r}) \epsilon_{x c}(n(\mathbf{r}))\right]}{\partial n(\mathbf{r})}
$$

The exchange-correlation energy per particle can be split as the sum of the exchange and the correlation parts as

$$
\epsilon_{x c}(n)=\epsilon_{x}(n)+\epsilon_{c}(n)
$$

The exchange part is given by Dirac expression for the exchange energy functional as [98, 104].

$$
\epsilon_{x}(n)=-C_{x} n(\mathbf{r})^{1 / 3}
$$

where

$$
C_{x}=\frac{3}{4}\left(\frac{3}{\pi}\right)^{1 / 3}
$$


Since the functional form of the correlation term is not known, it is determined by using Quantum Monte-Carlo simulations [98. By incorporating the spins, the exchange-correlation functional can be generalized as

$$
E_{x c}\left[n^{\uparrow}, n^{\downarrow}\right]=\int \epsilon_{x c}\left(n^{\uparrow}, n^{\downarrow}\right) n(\mathbf{r}) d^{3} \mathbf{r}
$$

This approximation is called Local Spin Density Approximation (LSDA).

\subsubsection{Generalized Gradient Approximation}

The generalized gradient approximation (GGA) is different from LDA in the sense that it incorporates the inhomogeneities in the electron density arising due to its variation in space by taking the gradient of the electron density in the expression of exchange-correlation energy functional. In GGA approximation, the exchangecorrelation energy functional can be written as

$$
E_{x c}^{G G A}[n]=\int f(n(\mathbf{r}), \nabla n) d^{3} \mathbf{r}
$$

If we incorporate spins, Equation 2.65 can be generalized as [105]

$$
E_{x c}^{G G A}\left[n^{\uparrow}, n^{\downarrow}\right]=\int f\left(n^{\uparrow}, n^{\downarrow}, \nabla n^{\uparrow}, \nabla n^{\downarrow}\right) d^{3} \mathbf{r}
$$


GGA exists in different forms. One of such forms is PBE (Perdew, Burke, and Ernzerhof)[105].

\subsubsection{Hybrid Functional}

In Hartree-Fock theory, the Coulomb term $J_{i j}$ and the exchange term $K_{i j}$ cancels each other in the expression of energy for $i=j$ as stated earlier. Therefore, there is no self-interaction error in Hartree-Fock theory. However, in Kohn-Sham DFT, the Coulomb repulsions between the electrons are treated exactly, but the exchange interactions are approximated. Therefore, they don't cancel exactly and leads to errors called self-interaction errors (SIE). Physically, SIE represents the interaction of an electron with itself and should be avoided in calculations. As a result of SIE, the local/semi-local functionals like LDA and GGA underestimate the energy gap in several systems like Group IV semiconductors. In such cases, hybrid functionals like HSE and B3LYP can be useful as these are formed in such a way that a part of the nonlocal exchange energy comes from the Hartree-Fock theory and the remaining from the other available local/semi-local functionals like LDA and PBE; the correlation part in hybrid functional comes entirely from the local/semi-local functional. The 
Hartree-Fock exact exchange energy term is given by 106$]$

$$
\begin{aligned}
E_{x}^{H F} & =-\frac{1}{2} \sum_{i} \sum_{j} K_{i j} \\
K_{i j} & =\iint \phi_{i}^{*}\left(\mathbf{r}_{1}\right) \phi_{j}^{*}\left(\mathbf{r}_{2}\right) \frac{1}{\left|\mathbf{r}_{1}-\mathbf{r}_{2}\right|} \phi_{j}\left(\mathbf{r}_{1}\right) \phi_{i}\left(\mathbf{r}_{2}\right) d^{3} \mathbf{r}_{1} d^{3} \mathbf{r}_{2}
\end{aligned}
$$

\section{HSE:}

One of the commonly used Hybrid functional is HSE proposed by Heyd-Scuseria-Ernzerhof. It splits the Hartree-Fock exchange interactions into two parts (i) short-range (SR) (ii) long-range (LR) and then avoids the LR Hartree Fock exchange component as these are slowly varying and are computationally expensive. The exchange-correlation energy in this approximation can be expressed as 107, 108]

$$
E_{x c}^{H S E}=a E_{x}^{H F, S R}(\omega)+(1-a) E_{x}^{P B E, S R}(\omega)+E_{x}^{P B E, L R}(\omega)+E_{c}^{P B E}
$$

In Equation 2.68, $a$ is the parameter that determines the amount of mixing of the two exchanges (HF and $\mathrm{PBE}$ ) and $\omega$ is the parameter that controls the extent of the short-range interactions. In Equation 2.68, the subscript $\mathrm{x}$ and c stand for exchange and correlation; the superscript SR and LR stand for short-range and long-range interactions respectively. There are two forms of HSE: (a) HSE06 for which $a=\frac{1}{4}$ and $\omega=0.2$ (b) HSE03 for which $a=\frac{1}{4}$ and $\omega=0.3$.

\section{B3LYP:}


Another widely used hybrid functional is B3LYP (Becke, three-parameter, Lee-YangParr) [106, 109, 110] in which the exchange-correlation energy functional can be expressed as

$$
E_{x c}^{B 3 L Y P}=E_{x c}^{L D A}+a_{0}\left(E_{x}^{H F}-E_{x}^{L D A}\right)+a_{x}\left(E_{x}^{G G A}-E_{x}^{L D A}\right)+a_{c}\left(E_{c}^{G G A}-E_{c}^{L D A}\right)
$$

where $a_{0}=0.20, a_{\mathrm{x}}=0.72$, and $a_{\mathrm{c}}=0.81$ are the mixing parameters.

\subsection{Bloch's Theorem and Plane-Wave Expansion}

Till now, we simplified the problem of the many-body system by decoupling the combined problem of the electrons and ions into two separate problems: one for the electrons and the other for the ions. We then split a single problem for $N_{e}$ electrons to $N_{e}$ single electron problems using both the WFT and DFT. We also said that by switching from WFT to DFT, the number of variables decreases from $3 N_{e}$ to just three. This was a huge simplification as it gave hope of solving many-body systems in computers. However, in a real system, we deal with many electrons and nuclei of the order of $10^{23}$. Therefore, it's almost impossible to calculate the wave functions for each of these electrons. Further, as the electronic wave function extends all over the crystal, the size of the basis sets needed to expand each of these electrons would 
be infinite [87]. To reduce the computational complexity of the problem, we make use of the fact that the system under study is periodic in nature; the electrons move in a periodic potential of the form $V(\mathbf{r})=V(\mathbf{r}+\mathbf{R})$, where $\mathbf{R}$ is the Bravais lattice vector. The periodicity of a crystal leads to the Bloch theorem which states that the solution of the SWE in a periodic potential with Hamiltonian of the form, $H=\frac{1}{2} \nabla^{2}+V(\mathbf{r})$, is the product of the plane wave and a function with the same periodicity as that of lattice[111, 112].

$$
\phi_{n k}(\mathbf{r})=f_{n k}(\mathbf{r}) e^{i \mathbf{k} \cdot \mathbf{r}}
$$

In Equation 2.70, $\mathbf{k}$ is a Bloch vector, $\mathrm{n}$ is the band index, and $f_{n}(\mathbf{r})$ is a function that has the periodicity of the lattice. It $\left(f_{n}(\mathbf{r})\right)$ is given by[111, 112]

$$
f_{n \mathbf{k}}(\mathbf{r})=f_{n \mathbf{k}}(\mathbf{r}+\mathbf{R})
$$

Translation of the Bloch wave function by a Bravais lattice vector $\mathrm{R}$ yields 111,112 ,

$$
\phi_{n \mathbf{k}}(\mathbf{r}+\mathbf{R})=\phi_{n \mathbf{k}}(\mathbf{r}) e^{i \mathbf{k} \cdot \mathbf{R}}
$$

The charge density is

$$
\left|\phi_{n \mathbf{k}}(\mathbf{r}+\mathbf{R})\right|^{2}=\left|\phi_{n \mathbf{k}}(\mathbf{r})\right|^{2}
$$

Equation 2.72 and 2.73 tells that although the wave function at a point $\mathbf{r}+\mathbf{R}$ will gain a phase of $e^{i \mathbf{k} \cdot \mathbf{R}}$ on translation by Bravais lattice vector $\mathbf{R}$, the charge density 
remains invariant. This suggests that we don't have to consider all the electrons in a crystal if we implement Bloch theorem and periodicity. We can concentrate only on the electrons in a unit cell to solve the problem of a many-electron system. The advantage of the Bloch theorem is therefore to reduce the problem of solving an infinite number of electrons in an infinite crystal (periodic) to the problem of solving a finite number of electrons in a unit cell[87]. Since $f_{n}(\mathbf{r})$ is periodic, it can be expanded in terms of Fourier series as the sum of discrete plane waves with wave vectors equal to reciprocal lattice vectors $\mathbf{G}$ of the crystal[111, 112].

$$
f_{n \mathbf{k}}(\mathbf{r})=\sum_{\mathbf{G}} c_{n \mathbf{G}} e^{i \mathbf{G} \cdot \mathbf{r}}
$$

Therefore, a single electronic wave function in Equation 2.70 can be expressed as the sum of plane waves[111, 112]:

$$
\phi_{n \mathbf{k}}(\mathbf{r})=\sum_{\mathbf{G}} c_{n \mathbf{k}+\mathbf{G}} e^{i(\mathbf{k}+\mathbf{G}) \cdot \mathbf{r}}
$$

In Equation 2.75, only those G's that are the reciprocal lattice vectors of the crystal such that $\mathbf{R} \cdot \mathbf{G}=2 \pi m$ are allowed and acceptable. Here, $\mathbf{R}$ is the direct lattice vector of the direct lattice. Therefore, there are only discrete G's due to lattice periodicity. $\phi_{n k}(\mathbf{r})$, in Equation 2.75, are the Kohn-Sham states. The Kohn-Sham equations can be solved in reciprocal space to obtain the orbitals $\phi_{n k}(\mathbf{r})$ and energy eigen values $\epsilon_{n k}$ for a given $\mathrm{k}$ and $\mathrm{n}$. Since any two wave vectors $\left(\mathbf{k}^{\prime}\right.$ and $\left.\mathbf{k}\right)$ satisfy the condition 
$\mathbf{k}^{\prime}=\mathbf{k}+\mathbf{G}$, the orbitals $\phi_{n k}(\mathbf{r})$ and energy eigen values (bands) $\epsilon_{n k}$ are periodic function in the reciprocal space[111, 112, implying that we only need to calculate the energy and orbitals in a single unit cell in the reciprocal space[111, 112] .

$$
\begin{array}{r}
\phi_{n, \mathbf{k}+\mathbf{G}}(\mathbf{r})=\phi_{n \mathbf{k}}(\mathbf{r}) \\
\epsilon_{n, \mathbf{k}+\mathbf{G}}=\epsilon_{n \mathbf{k}}
\end{array}
$$

$\mathbf{k}$, in Equation 2.76, is a continuous variable. Therefore, in the next section, we provide a way to discretize the k-space and obtain the observables of the system using a finite number of k-points in the reciprocal space.

\section{8 k-point Sampling}

Let us consider a periodic crystal (infinite) with $N_{1}, N_{2}$, and $N_{3}$ number of cells along the direction of primitive vectors $\mathbf{a}_{1}, \mathbf{a}_{2}$, and $\mathbf{a}_{3}$ respectively in real space. The total number of cells in the entire crystal is $N_{\text {cells }}=N_{1} \times N_{2} \times, N_{3}$. We impose Born-Von Karmen periodic boundary condition that the wave function entering one face of the crystal must match the wave function leaving the opposite face i.e.[112]

$$
\phi_{n \mathbf{k}}\left(\mathbf{r}+N_{i} \mathbf{a}_{i}\right)=\phi_{n \mathbf{k}}(\mathbf{r})
$$


Using Bloch theorem: $\phi_{n \mathbf{k}}\left(\mathbf{r}+N_{i} \mathbf{a}_{i}\right)=\phi_{n \mathbf{k}}(\mathbf{r}) e^{\left(i N_{i} \mathbf{k} \cdot \mathbf{a}_{i}\right)}$, Equation 2.77 becomes

$$
\begin{array}{r}
e^{\left(i N_{i} \mathbf{k} \cdot \mathbf{a}_{i}\right)}=1=e^{\left(i 2 \pi m_{i}\right)} \\
\Rightarrow \mathbf{k} \cdot \mathbf{a}_{i}=2 \pi \frac{m_{i}}{N_{i}}
\end{array}
$$

where $\mathrm{i}=1,2,3$ and $m_{i}$ are integers. This condition impose restriction on the values of $\mathbf{k}$ that we can take and makes sure that $\mathbf{k}$ is discrete in number. This allows us to define the Bloch vector $\mathbf{k}$ in the reciprocal space as

$$
\mathbf{k}=u_{1} \mathbf{b}_{1}+u_{2} \mathbf{b}_{2}+u_{3} \mathbf{b}_{3}
$$

where $u_{i}$ are numbers. Using $\mathbf{a}_{i} \cdot \mathbf{b}_{i}=2 \pi \delta_{i j}, u_{i}=\frac{m_{i}}{N_{i}}$. The allowed values of the Bloch vectors $\mathbf{k}$ are[112]

$$
\mathbf{k}=\sum_{i=1}^{3} \frac{m_{i}}{N_{i}} \mathbf{b}_{i}
$$

The elemental volume in reciprocal space is given by 112 ]

$$
\begin{aligned}
\Delta \mathbf{k} & =\frac{\mathbf{b}_{1}}{N_{1}} \cdot\left(\frac{\mathbf{b}_{2}}{N_{2}} \times \frac{\mathbf{b}_{3}}{N_{3}}\right) \\
& \Rightarrow \mathbf{b}_{1} \cdot\left(\mathbf{b}_{2} \times \mathbf{b}_{3}\right)=N_{\text {cells }} \times \Delta \mathbf{k}
\end{aligned}
$$

This means the volume $\left(\mathbf{b}_{1} \cdot\left(\mathbf{b}_{2} \times \mathbf{b}_{3}\right)\right)$ of a primitive cell in the reciprocal lattice is equal to the number of cells in real space times the spacing in k-space, implying that there are $N_{\text {cells }}$ points in a unit cell in the reciprocal space where each point is contributed by a unit cell in the real space. As said earlier, the condition: $\mathbf{k}^{\prime}=\mathbf{k}+\mathbf{G}$ 
demands that we don't have to go beyond the unit cell in reciprocal space to calculate the wave function and energy. This means the problem has now reduced from the problem of a continuous $\mathbf{k}$ in reciprocal lattice to a discrete $\mathbf{k}$ in a unit cell of the reciprocal lattice. But the allowed values of $\mathbf{k}$ are still infinite. Let's now reduce the number of k-points to a finite number.

In plane-wave based DFT, the electronic properties like charge density, DOS, energy etc. can be obtained by integrating over the Brillouin zone (BZ) as

$$
f(\mathbf{r})=\frac{1}{\Omega_{B Z}} \sum_{n} \int_{B Z} f_{n}(\mathbf{k}) d^{3} \mathbf{k}
$$

$\Omega_{B Z}$ is the cell volume in the Brillouin zone. For $\Delta k=0$ i.e. if two k-points in the $\mathrm{BZ}$ are close enough, the orbitals are almost identical indicating that the wave function over a region of k-space can be approximated by the wave function at a single k-point [87]. This means we do not need an infinite number of k-points in the reciprocal space to evaluate the above integral. We can replace the integral over the entire BZ by summation over the discrete but finite number of k-points by using the following transformation

$$
\frac{1}{\Omega_{B Z}} \int_{B Z} \rightarrow \sum_{k}^{B Z}
$$

Equation 2.82 then becomes

$$
f(\mathbf{r})=\sum_{n k}^{B Z} f_{n}(\mathbf{k})
$$


If the system under study has some point group symmetry, the irreducible BZ is just a subset of the entire BZ. In such cases, instead of summing over the entire Brillouin zone, we can perform weighted sum over the fewer k-points in the irreducible BZ as

$$
f(\mathbf{r})=\sum_{n k}^{I B Z} f_{n}(\mathbf{k}) w_{k}
$$

where, $w_{k}{ }^{\prime} s$ are weights. Now, one may ask: how do we choose the appropriate number of k-points in the BZ? To answer this, we do a convergence test with respect to the number of k-points. It may be noted that as the size of the unit cell in the direct lattice increases, the size of the BZ in the reciprocal lattice becomes smaller. This means we need fewer points to sample the BZ/IBZ for large supercells. This is a huge advantage as it allows the computations of large supercells possible in computers.

In this work, we have used Monkhorst pack [83, 113, 114] to sample the BZ in which the numbers $u_{i}$ in Equation 2.79 are given by

$$
\begin{array}{r}
u_{i}=\left(2 r_{i}-N_{i}-1\right) / 2 N_{i} \\
r_{i}=1,2,3, \ldots, N_{i} \\
i=1,2,3
\end{array}
$$

$N_{i}$ is an integer that defines the number of special k-points in the set. 


\subsection{Truncating Plane-Wave Basis Sets}

In Equation 2.75, we expressed the Kohn-Sham orbitals as the infinite sum of plane waves in the reciprocal lattice. It may be noted that the higher Fourier component $C_{n, k+G}$ in Equation 2.75 is much smaller for the plane waves with higher kinetic energy and therefore can be neglected[87]. This suggests that the expansion in Equation 2.75 can be truncated at some value of $|\mathbf{k}+\mathbf{G}|$. For this, we introduce a concept of kinetic energy cut-off for plane waves such that all the plane waves that satisfy the equation[115]

$$
\begin{aligned}
|\mathbf{k}+\mathbf{G}| & \leq \mathbf{G}_{c u t} \\
\Rightarrow \frac{1}{2}|\mathbf{k}+\mathbf{G}|^{2} & \leq \frac{1}{2} \mathbf{G}_{c u t}^{2} \\
\Rightarrow \frac{1}{2}|\mathbf{k}+\mathbf{G}|^{2} & \leq E_{c u t}
\end{aligned}
$$

are considered for the expansion in Equation 2.75. Equation 2.87 can be represented by a sphere (Figure 2.87) in reciprocal space within which all allowed points in the expansion of the plane waves lie. The number of plane waves within such sphere can be approximated in terms of $E_{c u t}$ by

$$
N_{P W}=\frac{V E_{c u t}^{\frac{3}{2}}}{6 \pi^{2}}
$$




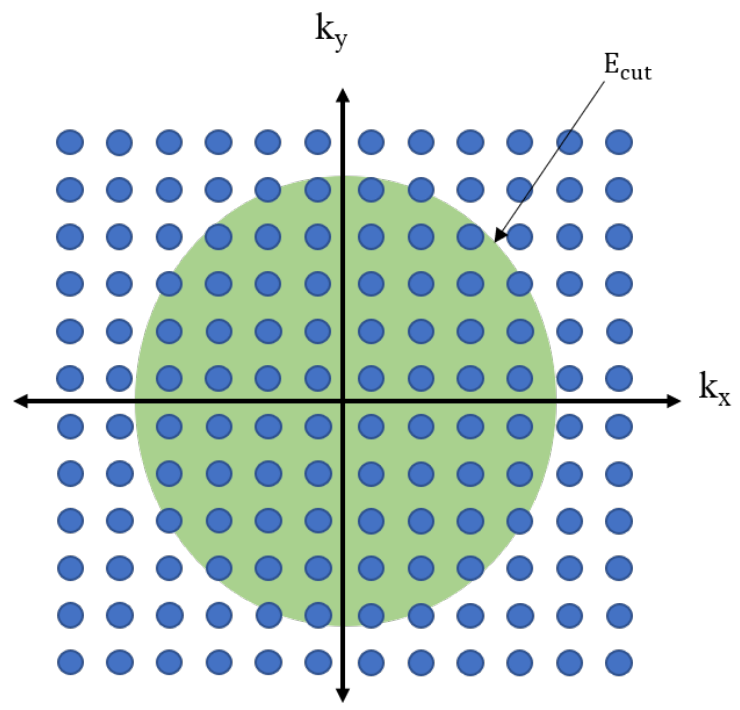

Figure 2.3: Planes waves can be represented by grids in reciprocal space; all plane waves within the cutoff energy are chosen for plane-wave expansion.

where $\mathrm{V}$ is the volume of the unit cell in the real space. Such truncation might lead to errors in the computed values of observables. However, these errors can be minimized by increasing the value of cut-off energy. It is worth noting that it may not be always feasible to increase the cut-off energy especially for a system with a large number of electrons. Then one can ask again: how do we select the relevant value of $E_{\text {cut }}$ for your calculations? To know this, we do an energy convergence test with respect to the $E_{\text {cut }}$. 


\subsection{Pseudopotential Approximation}

In previous sections, we reduced the complexity of solving the problem of the manyelectron system by introducing the concept of periodicity of the crystal, k-point sampling, and kinetic energy cut-off. In this section, we go a step further and lessen the number of plane waves required in our calculations by using the notion of pseudopotential. In a real many-body system, the core electrons feel strong nuclear potential

(a)

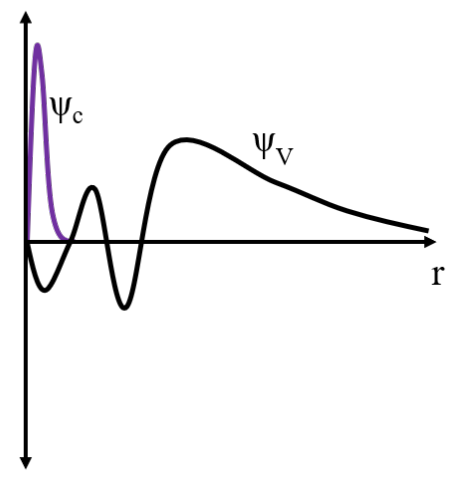

(b)

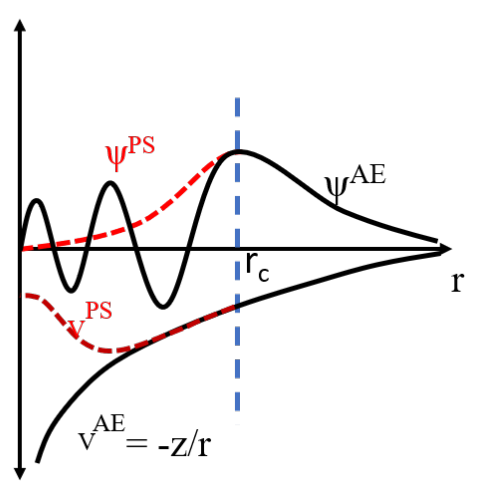

Figure 2.4: Schematic showing (a) all electron wave functions of the core and valence electrons (b) pseudo wave function and pseudo potential below and above the cut off radius $r_{c}$ and the corresponding all electron wave function and potential.

due to the nucleus. It has two consequences: (i) the core electrons wave functions are localized near the nucleus (ii) the valence electron wave functions have a lot of wiggles near the nucleus as these have to be orthogonal to the core electrons wave functions [87]. If we want to represent such wave functions as the linear combination 
of plane waves, we might need a basis with a large number of plane waves. This makes all-electron computations nearly impossible in computers. However, it is the valence electrons in materials that take part in bonding and determine their physical properties. This allows us to replace the core electrons and the nucleus with some effective potential, also called pseudopotential, such that only the valence electrons experience this effective potential. Since the valence electrons wave functions $\left(\psi^{\mathrm{AE}}\right)$ have a lot of wiggles near the nucleus and are much smoother away from the nucleus, we can define a cut-off radius $r_{c}$. Below $r_{c}$, the pseudopotential $\left(\mathrm{V}^{\mathrm{PS}}\right)$ and pseudowave function $\left(\psi^{\mathrm{PS}}\right)$ are much smoother than the nuclear potential $\left(\mathrm{V}^{\mathrm{AE}}\right)$ and valence electron wave functions $\left(\psi^{\mathrm{AE}}\right)\left[87\right.$ ]. Above $r_{c}$, they are identical (Figure 2.4) [87]. The use of pseudopotential increases the efficiency of our calculations due to the following two reasons (i) we have less number of electrons for expansion (ii) we replace wiggles in valence electron wave function by a much smoother function within the cut-off radius $r_{c}$. This also means that the plane waves required in our calculations decrease by a lot.

While constructing a pseudopotential, we should note the followings [87, 116]:

(i) Above $r_{c}$, the pseudo wave function $\left(\psi^{\mathrm{PS}}\right)$ and all-electron wave function $\left(\psi^{\mathrm{AE}}\right)$ should be identical.

(ii) The pseudo wave function $\left(\psi^{\mathrm{PS}}\right)$ should be a smooth function and should not contain any nodes.

(iii) The all-electron eigen values should be similar to the pseudo eigenvalues. 
(iv) Within $r_{c}$, the charge enclosed by the valence wave function and the pseudo wave function should satisfy the condition

$$
\int_{0}^{r_{c}}\left|\psi_{l}^{A E}(\mathbf{r})\right|^{2} d^{3} \mathbf{r}=\int_{0}^{r_{c}}\left|\psi_{l}^{P S}(\mathbf{r})\right|^{2} d^{3} \mathbf{r}
$$

(v) For $r>r_{c}$, the logarithmic derivatives of the pseudo wave function and its first energy derivatives should be identical to the corresponding derivatives for the allelectron wave function.

In Equation 2.89, $l$ represents the angular momentum of the system. The pseudopotentials that satisfy the Equation 2.89, also called norm-conserving criteria, are the norm-conserving pseudopotentials[116]. However, there are several systems (like O $2 \mathrm{p}$ and $\mathrm{Ni} 3 \mathrm{~d}$ ) where it is not possible to create the pseudo wave functions which are softer than all-electron wave functions using the norm-conservation requirement [117]. Further, the norm-conserving pseudopotentials are computationally expensive for systems involving transition metal and rare-earth atoms[118]. In such cases, Ultra-soft pseudopotential can be useful which was proposed by Vanderbilt in 1990[117]. In this type of pseudopotential, the norm-conserving condition is relaxed so that the pseudo wave function becomes much smoother. This has the advantage that the number of plane waves required in our calculations can be decreased significantly (i.e. kinetic energy cut-off can be lowered) thereby increasing the computational efficiency. 


\subsection{Plane-Wave Representation of Kohn-Sham Equations}

Using the concept of the periodicity of the lattice, k-point sampling, symmetry of

the crystal, plane wave cut-off, and pseudopotentials, we have significantly lower the number of plane waves in our caclulations. Therefore we diagonalize much smaller matrix of size $N_{b} \times N_{b}$. Using plane wave expansion of orbitals in Equation 2.57, the Kohn-Sham equation becomes [87]

$$
\begin{array}{r}
\sum_{\mathbf{G}^{\prime}}\left[\frac{1}{2}|\mathbf{k}+\mathbf{G}|^{2} \delta_{\mathbf{G G}^{\prime}}+V_{e x t}\left(\mathbf{G}-\mathbf{G}^{\prime}\right)+V_{H}\left(\mathbf{G}-\mathbf{G}^{\prime}\right)+V_{x c}\left(\mathbf{G}-\mathbf{G}^{\prime}\right)\right] c_{i, \mathbf{k}+\mathbf{G}^{\prime}} \\
=\epsilon_{i} c_{i, \mathbf{k}+\mathbf{G}}
\end{array}
$$

As stated earlier, $V_{\text {ext }}(\mathbf{G})=V_{\text {ion }}(\mathbf{G})$. Equation 2.90 can be solved by diagonalizing the Hamiltonian. It may be noted that the size of the matrix is determined by the cut-off energy.

\subsection{Collinear and Noncollinear Magnetism}

In this work, we have studied both the collinear and non-collinear magnetism. In collinear magnetism, the magnetization vector $\vec{m}(\mathbf{r})=m(\mathbf{r}) \hat{z}$. Here, the unit vector 
$\hat{z}$ may be either parallel or antiparallel to a given direction $\hat{z}$, indicating the magnetization is either parallel or antiparallel to $\hat{z}$. In noncollinear magnetism, the magnetization $\vec{m}(\mathbf{r})$ is a continuous vector variable of position $\mathbf{r}[119]$. It is the spin-orbit interactions that couples the magnetization to a crystal lattice. Once the spin-orbit coupling (SOC) is turned on, rotations of these spin magnetic moments in different directions yield different energy[115]. So the basic variables in spin DFT are the scalar electron density and the magnetization density (a vector) [120]. Here instead of these 4 variables, we use a $2 \times 2$ density matrix with elements $n^{\alpha \beta}(\mathbf{r})$ to express spin DFT[119, 120].

$$
\operatorname{Tr}\left[n^{\alpha \beta}(\mathbf{r})\right] \equiv n_{T r}(\mathbf{r})=\sum_{\alpha} n^{\alpha \alpha}(\mathbf{r})
$$

The total density matrix is [119]

$$
n^{\alpha \beta}(\mathbf{r})=\left[n_{T r}(\mathbf{r}) \delta_{\alpha \beta}(\mathbf{r})+\vec{m}(\mathbf{r}) \cdot \vec{\sigma}_{\alpha \beta}(\mathbf{r})\right] / 2
$$

The magnetization density is [119]

$$
\vec{m}(\mathbf{r})=\sum_{\alpha \beta} n^{\alpha \beta}(\mathbf{r}) \cdot \vec{\sigma}_{\alpha \beta}(\mathbf{r})
$$


Here, $\sigma$ is Pauli's spin matrices. The Kohn-Sham energy functional can be written as 119, 121]

$$
\begin{aligned}
E & =\sum_{\alpha} \sum_{n} f_{n}\left\langle\Psi_{n}^{\alpha}\left|-\frac{1}{2} \Delta\right| \Psi_{n}^{\alpha}\right\rangle+\int V_{e x t}(\mathbf{r}) n_{T r}(\mathbf{r}) d^{3} \mathbf{r} \\
& +\frac{1}{2} \iint \frac{n_{T r}(\mathbf{r}) n_{T r}\left(\mathbf{r}^{\prime}\right)}{\left|\mathbf{r}-\mathbf{r}^{\prime}\right|} d^{3} \mathbf{r} d^{3} \mathbf{r}^{\prime}+E_{x c}[\overleftrightarrow{n}(\mathbf{r})]
\end{aligned}
$$

The Kohn-Sham equations can be expressed as [121]

$$
\sum_{\beta} H^{\alpha \beta}\left|\Psi_{n}^{\beta}\right\rangle=\epsilon_{n} S^{\alpha \alpha}\left|\Psi_{n}^{\alpha}\right\rangle
$$

where[121]

$$
H^{\alpha \beta}=-\frac{1}{2} \Delta \delta_{\alpha \beta}+V_{e x t}(\mathbf{r}) \delta_{\alpha \beta}+\frac{1}{2} \int \frac{n_{T r}\left(\mathbf{r}^{\prime}\right)}{\left|\mathbf{r}-\mathbf{r}^{\prime}\right|} d^{3} \mathbf{r}^{\prime} \delta_{\alpha \beta}+V_{x c}^{\alpha \beta}[\overleftrightarrow{n}(\mathbf{r})](\mathbf{r})
$$

In matrix form, we have[121]

$$
\left[\begin{array}{cc}
H^{\alpha \alpha} & V_{x c}^{\alpha \beta} \\
V_{x c}^{\beta \alpha} & H^{\beta \beta}
\end{array}\right]\left[\begin{array}{l}
\left|\Psi_{n}^{\alpha}\right\rangle \\
\left|\Psi_{n}^{\beta}\right\rangle
\end{array}\right]=\epsilon_{n}\left[\begin{array}{c}
\left|\Psi_{n}^{\alpha}\right\rangle \\
\left|\Psi_{n}^{\beta}\right\rangle
\end{array}\right]
$$

where

$$
V_{x c}^{\alpha \beta}[\overleftrightarrow{n}(\mathbf{r})]=\frac{\delta E_{x c}[\overleftrightarrow{n}(\mathbf{r})]}{\delta n^{\beta \alpha}(\mathbf{r})}
$$

$\overleftrightarrow{n}$ is $2 \times 2$ matrix same as $n^{\alpha \beta}$. It may be noted that for $\vec{m}(\mathbf{r})=m(\mathbf{r}) \hat{z}, \overleftrightarrow{n}$ is 
diagonal and an approximation to the exchange correlation functional $E_{x c}[\overleftrightarrow{n}]$ is well founded[121]. Therefore, we need to diagonalize $n^{\alpha \beta}$. This can be done by using the spin-1/2 rotation matrices $U(\mathbf{r})[121]$.

$$
\sum_{\alpha \beta} U_{i \alpha}(\mathbf{r}) n^{\beta \alpha}(\mathbf{r}) U_{\beta j}^{+}(\mathbf{r})=\delta_{i j} n_{i}(\mathbf{r})
$$

$n_{i}(\mathbf{r})$ is the eigen values that depends on the position $\mathbf{r}[120$. The exchange-correlation potential can be written as

$$
V_{x c}^{\alpha \beta}(\mathbf{r})=\frac{1}{2}\left[\frac{\delta E_{x c}}{\delta n^{1}(\mathbf{r})}+\frac{\delta E_{x c}}{\delta n^{2}(\mathbf{r})}\right] \delta_{\alpha \beta}+\frac{1}{2}\left[\frac{\delta E_{x c}}{\delta n^{1}(\mathbf{r})}-\frac{\delta E_{x c}}{\delta n^{2}(\mathbf{r})}\right]\left(U^{+}(\mathbf{r}) \sigma_{z} U(\mathbf{r})\right)_{\alpha \beta}
$$

Equivalently, using 121$]$

$$
\begin{gathered}
n^{\uparrow}(\mathbf{r})=\frac{1}{2}\left[n_{T r}(\mathbf{r})+|m(\mathbf{r})|\right] \\
n^{\downarrow}(\mathbf{r})=\frac{1}{2}\left[n_{T r}(\mathbf{r})-|m(\mathbf{r})|\right] \\
m \hat{(\mathbf{r})}=\frac{m(\mathbf{r})}{|m(\mathbf{r})|}
\end{gathered}
$$

the exchange-correlation potential can be approximated as [121]

$$
\begin{gathered}
V_{x c}^{\alpha \beta}(\mathbf{r})=\frac{1}{2}\left[\frac{\delta E_{x c}}{\delta n^{\uparrow}(\mathbf{r})}+\frac{\delta E_{x c}}{\delta n^{\downarrow}(\mathbf{r})}\right] \delta_{\alpha \beta}+\frac{1}{2}\left[\frac{\delta E_{x c}}{\delta n^{\uparrow}(\mathbf{r})}-\frac{\delta E_{x c}}{\delta n^{\downarrow}(\mathbf{r})}\right] \hat{m(\mathbf{r}) \cdot \vec{\sigma}_{\alpha \beta}} \\
E_{x c}=\int n_{T r}(\mathbf{r}) \epsilon_{x c}\left[n_{\uparrow}(\mathbf{r}), n_{\downarrow}(\mathbf{r})\right] d^{3} \mathbf{r}
\end{gathered}
$$


In collinear case, there is existence of a common magnetization axis for all the atoms and spin- $1 / 2$ rotation matrices are independent on the position $\mathbf{r}[120]$. In such cases, all the observables including the energy are functional of the density and the magnitude of magnetization $|m(\mathbf{r})|$ instead of the magnetization vector $\vec{m}(\mathbf{r})[120]$. The spin-up and spin-down spin densities can be obtained using the spin-up and spin-down orbitals using 120 ]

$$
\begin{aligned}
& n^{\uparrow}(\mathbf{r})=\sum_{i=1}^{N_{e}^{\uparrow}}\left|\phi_{i}^{\uparrow}(\mathbf{r})\right|^{2} \\
& n^{\downarrow}(\mathbf{r})=\sum_{i=1}^{N_{e}^{\downarrow}}\left|\phi_{i}^{\downarrow}(\mathbf{r})\right|^{2}
\end{aligned}
$$

The Kohn-Sham equations for collinear case are 120 .

$$
\left[-\frac{1}{2} \nabla^{2}+V^{K S, \sigma}(\mathbf{r})\right] \phi_{i}^{\sigma}(\mathbf{r})=\epsilon_{i}^{\sigma} \phi_{i}^{\sigma}(\mathbf{r})
$$

where, $\sigma=\uparrow$ or $\downarrow$ with the effective potential given by[120]

$$
V^{K S, \sigma}(\mathbf{r})=\int \frac{n\left(\mathbf{r}^{\prime}\right)}{\left|\mathbf{r}-\mathbf{r}^{\prime}\right|} d^{3} \mathbf{r}^{\prime}+V_{e x t}^{\sigma}(\mathbf{r})+V_{x c}^{\sigma}(\mathbf{r})
$$

It is worth noting that the external potential contains a field terms $-\left( \pm \mu_{B} B\right)$ in the presence of the magnetic field; the negative sign in front indicates that spin-up electrons (majority spin carriers) are energetically favorable than spin-down electrons 
(minority spin carriers) [120]. The exchange-correlation potential can be expressed as

$$
V_{x c}^{\sigma}(\mathbf{r})=\frac{\delta E_{x c}\left[n^{\uparrow}(\mathbf{r}), n^{\downarrow}(\mathbf{r})\right]}{\delta n^{\sigma}(\mathbf{r})}
$$

The total density can be written as 120

$$
n(\mathbf{r})=n^{\uparrow}(\mathbf{r})+n^{\downarrow}(\mathbf{r})
$$

The magnetization density is 120 ]

$$
m(\mathbf{r})=n^{\uparrow}(\mathbf{r})-n^{\downarrow}(\mathbf{r})
$$

The local magnetic moment $M$ for collinear case is obtained by the integration of the magnetization density over volume $\Omega[120]$

$$
M=\int_{\Omega} m(\mathbf{r}) d^{3} \mathbf{r}
$$

It may be noted that the collinear magnetic ordering may be ferromagnetic, antiferromagnetic, and ferrimagnetic states. 


\subsection{Projector Augmented Wave Method}

In augmented-wave methods, we split the electronic wave function into two parts by defining a cut-off radius $r_{c}$ around the atom. Below the sphere defined by the cut-off radius $r_{c}$, the wave function is represented by the partial wave expansion. Outside the sphere, the wave function is expressed as a linear combination of the functions (like plane waves) in a basis set. The boundary condition then demands that these wave functions and their derivatives must match at the boundary $r_{c}[122]$. PAW method uses the idea of Linear-Augmented-plane-wave (LAPW) method and Vanderbilt Ultrasoft pseudopotential (USSP) method. It was proposed by Blöch[122]. In the PAW method, there exists a linear transformation[118, 122, 123]

$$
\tau=1+\sum_{i}\left(\left|\phi_{i}\right\rangle-\left|\tilde{\phi}_{i}\right\rangle\right)\left\langle\tilde{p}_{i}\right|
$$

that allows us to obtain the all-electron (AE) wave function $\left|\psi_{n}\right\rangle$ from the pseudo (PS) wave function $\left|\tilde{\psi}_{n}\right\rangle$ using[118, 122, 123$]$

$$
\left|\psi_{n}\right\rangle=\left|\tilde{\psi}_{n}\right\rangle+\sum_{i}\left(\left|\phi_{i}\right\rangle-\left|\tilde{\phi}_{i}\right\rangle\right)\left\langle\tilde{p}_{i} \mid \tilde{\Psi}\right\rangle
$$

In Equations 2.108 and 2.109, i represents the atomic sites, $\left|\phi_{i}\right\rangle$ and $\left|\tilde{\phi}_{i}\right\rangle$ represents the all-electron (AE) and pseudo(PS) partial waves; the all-electron (AE) partial waves 
$\left|\phi_{i}\right\rangle$ are the solutions of the radial part of the SWE. Outside $r_{c},\left|\phi_{i}\right\rangle=\left|\tilde{\phi}_{i}\right\rangle$. Within $r_{c}$, $\left|\tilde{\phi}_{i}\right\rangle$ is matched onto $\left|\phi_{i}\right\rangle$. The pseudo wave function $\left|\tilde{\psi}_{n}\right\rangle$ are expanded as the linear combination of the plane waves in reciprocal space. $\tilde{p}_{i}$ are the projector function that satisfies the orthonormality condition $\left\langle\tilde{p}_{i} \mid \phi_{j}\right\rangle=\delta_{i j}$.

The PS operator form of the quasi local operators like kinetic energy within PAW formalism is [122]

$$
\begin{aligned}
\tilde{A} & =\tau^{\dagger} A \tau \\
& =A+\sum_{i j}\left|\tilde{p}_{i}\right\rangle\left(\left\langle\phi_{i}|A| \phi_{j}\right\rangle-\left\langle\tilde{\phi}_{i}|A| \tilde{\phi}_{j}\right\rangle\right)\left\langle\tilde{p}_{j}\right|
\end{aligned}
$$

If the operator is non-local, we have to add $\Delta A$ to the Equation 2.110]122], where

$$
\begin{aligned}
\Delta A & =\sum_{i}\left|\tilde{p}_{i}\right\rangle\left(\left\langle\phi_{i}\right|-\left\langle\tilde{\phi}_{i}\right|\right) A\left(1-\sum_{j}\left|\tilde{\phi}_{i}\right\rangle\left\langle\tilde{p}_{j}\right|\right) \\
& +\left(1-\left|\tilde{p}_{j}\right\rangle\left\langle\tilde{\phi}_{j}\right|\right) A\left(\left|\phi_{i}\right\rangle-\left|\tilde{\phi}_{i}\right\rangle\right)\left\langle\tilde{p}_{i}\right|
\end{aligned}
$$

\subsection{Spin-Orbit Coupling in PAW Method}

The spin-orbit coupling (SOC) is the interaction between the spin motion (represented by the Pauli matrices $\vec{\sigma}$ ) of an electron with its orbital motion (represented by the

orbital angular momentum $\vec{L}$ ). The effect of the spin-orbit interaction is to add an 
additional interaction term proportional to $\vec{\sigma} \cdot \vec{L}$ to the non-relativistic Hamiltonian as discussed before. Here, we will discuss the SOC implementation in the PAW method. As SOC is a relativistic effect, it is stronger closer to the nucleus (within PAW sphere) and is negligible away from the nucleus (exterior to the PAW sphere). This means there is the cancellation of the first and third term in Equation 2.110 123]. Therefore, the Hamiltonian (in the PAW formalism) that incorporates the effects of SOC can be expressed as 123

$$
\tilde{H}_{S O}=\sum_{i j}\left|p_{i}\right\rangle\left\langle\phi_{i}\left|H_{S O}\right| \phi_{j}\right\rangle\left\langle p_{j}\right|
$$

where

$$
H_{S O}^{\alpha \beta}=\frac{\hbar^{2}}{\left(2 m_{e} c\right)^{2}} \frac{K(\mathbf{r})}{r} \frac{d V(\mathbf{r})}{d r} \vec{\sigma}^{\alpha \beta} \cdot \vec{L}
$$

Here,

$$
K(\mathbf{r})=\left(1-\frac{V(\mathbf{r})}{2 m_{e} c^{2}}\right)^{-2}
$$

In Equation $2.113, \vec{\sigma}^{\alpha \beta} \cdot \vec{L}$ represents the interaction between electron orbital motion with its spin motion as said before. $\mathrm{V}(\mathrm{r})$, in Equation 2.113, is the effective AE potential within the PAW sphere.

Using, $\phi_{i}(\mathbf{r})=R_{i}(|\mathbf{r}|) Y_{l_{i} m_{i}}(\hat{\mathbf{r}})$, in Equation 2.113, we get[123]

$$
\tilde{H}_{S O}^{\alpha \beta}=\frac{\hbar^{2}}{\left(2 m_{e} c\right)^{2}} \sum_{i j}\left|\tilde{p}_{i}\right\rangle R_{i j} \vec{\sigma}_{\alpha \beta} \cdot \overrightarrow{L_{i j}}\left\langle\tilde{p}_{j}\right|
$$


$Y_{l_{i} m_{i}}$ in Equation 2.113 are the spherical harmonics. In Equation 2.113,

$$
\begin{array}{r}
R_{i j}=4 \pi \int_{0}^{r_{c}} R_{i}(\mathbf{r}) \frac{K(\mathbf{r})}{r} \frac{d V(\mathbf{r})}{d r} R_{j}(\mathbf{r}) d^{3} \mathbf{r} \\
\vec{L}_{i j}=\left\langle Y_{l_{i} m_{i}}|\vec{L}| Y_{l_{j} m_{j}}\right\rangle
\end{array}
$$

We can obtain the effect of the $\tilde{H}_{S O}^{\alpha \beta}$ on the PS orbitals using [123]

$$
\left|\tilde{\psi}_{n}^{\alpha}\right\rangle=\sum_{\alpha \beta} \tilde{H}_{S O}^{\alpha \beta}\left|\tilde{\psi}_{n}^{\beta}\right\rangle
$$

In Equation 2.117, $\alpha$ and $\beta$ represent the spin-up and spin-down components of the spinor [123]. 



\section{Chapter 3}

\section{Quantum Transport in a Nanoscale}

\section{Junction}

In this chapter, I will discuss the formalism of the charge and spin transport theory that has been used to calculate the transmission function and current in this thesis. Since the system under study is a one-dimensional system with a length scale of the order of a few nanometers, the quantum confinement effects are dominant leading to the discretization of energy levels. Therefore, the classical transport theory is inadequate to describe the transport phenomena in our system, suggesting the necessity to include the quantum nature of the electron in our theory to study its transport properties [2, 124, 125]. Let's begin this chapter by discussing the characteristics length scales that enter the picture when studying physics at a nanoscale level. 


\subsection{Fundamental Length Scales}

In this thesis, we want to study electron transport in a nanoscale junction. Being Fermions, these electrons obey Pauli's exclusion principle. At T=0K, electrons occupy the states up to a Fermi level (characterized by Fermi energy $E_{F}$, Fermi wave vector $k_{F}$, and Fermi velocity $v_{F}$ ) so that all the states below it are filled and above it are empty. It may be noted that only the electrons that are in the neighborhood of $E_{F}$ make a significant contribution to the transport in solids[126]. This also implies that the electrons with the characteristics wavelength of the order of Fermi wavelength $\lambda_{F}$ are of physical importance when studying the electron transport at a nanoscale level. The Fermi wavelength of a two-dimensional electron gas can be written in terms of its Fermi wave vector $\left(k_{F}\right)$ and electron density $\left(n_{s}\right)$ as [124, 125, 126]

$$
\begin{aligned}
\lambda_{F} & =\frac{2 \pi}{k_{F}} \\
& =\sqrt{2 \pi / n_{s}}
\end{aligned}
$$

The quantum effects are dominant if electronic vibrations in scattering potentials arising due to disorder or lattice vibrations (phonon induced scattering of electrons) in solids are of the same order as that of the Fermi wavelength $\left(\lambda_{F}\right)[126]$. The scattering of an electron in scattering potentials may be elastic or inelastic. In an elastic scattering, both the energy and phase of an electron is conserved. For elastic 
scattering processes, we can introduce a length scale called elastic mean free path $\left(l_{m}\right)$ which is defined as the average distance covered by an electron during two consecutive elastic scatterings. It can be expressed in terms of Fermi velocity $\left(v_{F}\right)$ and momentum relaxation time $\left(\tau_{m}\right)$ as [124, 125, 126]

$$
l_{m}=v_{F} \tau_{m}
$$

If the channel length $\left(l_{N}\right)$ and elastic mean free path $\left(l_{m}\right)$ obey the condition $l_{N}<l_{m}$, electrons move freely without being scattered. In this case, the electron transport becomes ballistic. On the other hand, if $l_{N}>l_{m}$, the charge carriers are scattered during transport and the transport becomes diffusive. In the second type of scattering process, also called inelastic scattering, the energy is not conserved. In this case, the phase of an electronic wave function is also not conserved. Such dephasing of an electronic wave function (accompanied by the energy change) when an electron comes across an inelastic scattering center can be quantified by a length scale called phase coherence length $l_{\phi}$ and is defined as the distance traveled by an electron between two consecutive inelastic scattering processes. Mathematically, it is given by the relation[124, 125, 126 ,

$$
l_{\phi}=v_{F} \tau_{\phi}
$$


In Equation 3.3, $v_{F}$ and $\tau_{\phi}$ are the Fermi velocity and the phase relaxation time. The knowledge of the phase coherence length $l_{\phi}$ determines the nature of transport through the nanoscale junction. If $l_{N}<l_{\phi}$, the quantum interference effects come into existence and electrons are transported coherently through the nanoscale junction [124, 125, 126]. On the other hand, if $l_{N}>l_{\phi}$, no quantum interference effects occur within the device $124,125,126$. Any fluctuations in the potential can cause the phase of an electronic wave function to relax; at a low temperature, such fluctuations in potential occur due to electron-electron interactions in solids.

Here, we are interested in studying the spin transport phenomena in a nanoscale device. The length scale that enters into the picture when studying spin transport phenomena is the spin diffusion length. It is defined as the distance over which a spin carrier retains the memory of its initial spin orientation intact during its transport through the channel. It is given by 126$]$

$$
l_{s d}=\sqrt{D \tau^{\sigma}}
$$

$\mathrm{D}$ and $\tau^{\sigma}$, in Equation 3.4, are the diffusion constant and spin relaxation time; $\sigma$ corresponds to spin-up or spin-down electrons. The spin diffusion length is determined by the strength of the spin-orbit and hyperfine interactions in materials and is usually larger in materials with a small atomic number[47]. For carbon nanotubes, $l_{s d} \sim 130$ $\mathrm{nm}[127]$. 


\subsection{Modeling of a Nanoscale Device}

The schematic of a prototypical nanowire junction is presented in Figure 3.1. It consists up of a finite segment of a one-dimensional nanowire placed in between two semiinfinite electrodes. The electrons are free to move in the $\mathrm{x}$-direction (the nanowire axis) and confined in transverse directions (y- and z-direction), suggesting the discretization of energy levels of these electrons in the nanowire. When a nanowire with such discrete energy levels is sandwiched between two electrodes that have different values of the chemical potential, electrons will flow from the electrode with higher chemical potential (called source) to the electrode with lower chemical potential (called drain); the pathway for such flow is provided by the nanowire channel. Such motion of the electrons from the source to the drain will give rise to current.

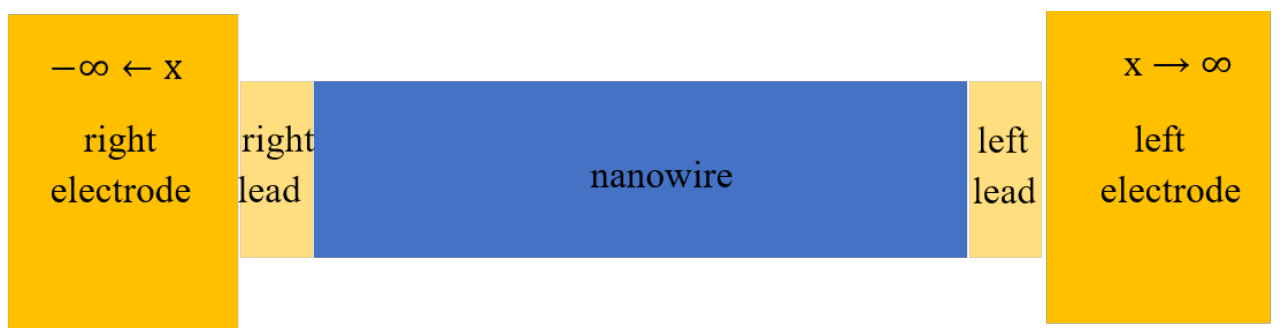

Figure 3.1: A schematic showing a two terminal nanowire junction; the nanowire together with the leads form an active scattering region.

Here, we make the following two assumptions: (a) the current through the nanowire 
junction is constant in time (b) the energy levels in the nanowire channel are stationary. To function as a device, the nanowire and the semi-infinite electrodes must exchange electrons and energy between them. The nanowire together with the semiinfinite electrodes forms an open system. For modeling purposes, we partition the open junction into three parts: (a) the spacer (channel) that includes nanowire of a finite length (b) the lead that contains a finite number of the metal atoms and (c) the unperturbed electrode part that retains the bulk nature of the electrode; this part acts as a source of electrons on one side and sink on the other side when the nanowire junction is in a non-equilibrium situation. The rationale behind taking a finite number of atoms in the lead is because it makes calculations of the coupling matrices between the lead-nanowire junction possible in computers. These leads are strongly coupled to the spacer/channel and together they form an active scattering region. The transport of carriers in a nanoscale junction is a non-equilibrium statistical phenomenon that can be achieved by the application of an electric field. Under this situation, the chemical potential of the left and the right electrodes are different and are given by

$$
\mu_{L, R}=E_{F} \mp V_{L, R}
$$

In Equation 3.5. $E_{F}$ and $V_{L, R}$ are the Fermi energy and voltage drop at the electrodes. In our calculations, we have determined these voltages self-consistently using a real space orbital-based DFT as implemented in Gaussian[128]. For this, we have calculated the average potentials of all the atoms at the left and right leads separately 
for each value of the applied electric field. From these average potentials, we have subtracted the average potential at zero bias. Then the source to drain voltage $V_{S D}$ at each field is obtained by calculating the difference between $V_{L}$ and $V_{R}$. It may be noted that at equilibrium, the potential at the left and the right electrode must satisfy the condition: $V_{L}=V_{R}=0$. If we are studying the gating effect on this nanowire junction, we do it by the application of an electric field (also called gate field) perpendicular to the nanowire axis.

\subsection{Landauer Formalism to Study Electron Trans- port}

Let's now discuss the Landauer formalism[129, 130] of electron transport that expresses the total current through the nanowire junction in terms of the transmission function. This formalism is valid in the regime of coherent electron transport that happens when the dimension of the device is less than the phase coherence length of the electron i.e. if $l_{d} \leq L_{\phi}[124,125,129,130]$. To derive an expression of the current, we consider a nanoscale junction as in Figure 3.1 and assume identical leads on either side of the scattering region. Since electrons are fermions, their distributions at the 
left and the right electrodes is given by Fermi-Dirac distribution as [124, 125]

$$
f_{L, R}(E)=\frac{1}{e^{\left(E-\mu_{L, R}\right) / k_{B} T}+1}
$$

In Equation 3.6, $\mu_{L}$ is the chemical potential of the left electrode; $\mu_{R}$ is the chemical potential for the right electrode. These chemical potentials are related to the bias voltage $\mathrm{V}$ by the mathematical relation $[124,125]$

$$
V=\frac{\mu_{L}-\mu_{R}}{e}
$$

where e is the charge of an electron. In the junction shown in Figure 3.1, the electrons move freely in the $\mathrm{x}$-direction, whereas they are confined in $\mathrm{y}$ and $\mathrm{z}$ directions. Such quantum confinement of electrons in the nanowire junction leads to the discretization of energy levels as discussed earlier. The Hamiltonian for the active scattering region is given by 124,125

$$
H_{S}=-\frac{\hbar^{2}}{2 m} \nabla^{2}+V(r)
$$

In the limit of $x \rightarrow \pm \infty$ (asymptotic condition), we can approximate the Hamiltonian in Equation 3.8 as 124,125

$$
\begin{gathered}
\lim _{x \rightarrow-\infty} H_{S}=-\frac{\hbar^{2}}{2 m} \nabla^{2}+V_{L}(r) \equiv H_{L} \\
\lim _{x \rightarrow-\infty} H_{S}=\frac{-\hbar^{2}}{2 m} \nabla^{2}+V_{R}(r) \equiv H_{R}
\end{gathered}
$$


where $H_{L}$ and $H_{R}$ are the Hamiltonian for the left and right electrode respectively. Let's first consider the SWE for the left electrode (with Hamiltonian $H_{L}$ ) [124, 125]

$$
\left[-\frac{\hbar^{2}}{2 m} \nabla^{2}+V_{L}(r)\right] \psi_{n k}(r)=E \psi_{n k}(r)
$$

The general solution of the SWE in Equation 3.11 can be written as the product of the longitudinal and transverse components as [124, 125]

$$
\psi_{n k}(r)=\sqrt{\frac{1}{L_{x}}} u_{n}\left(r_{\perp}\right) e^{i k x}, \quad-\infty<k<+\infty
$$

The energy eigen values are given by 124,125

$$
E_{n}(k)=\epsilon_{n}+\frac{\hbar^{2} k^{2}}{2 m}
$$

Since the eigen states in Equation 3.12 has a component of the form $\left(e^{i k x}\right)$, an electron has a non-zero probability density throughout the space. It is possible that the eigen value problem in Equation 3.11 has a decaying solution, but their contribution to the probability density is negligible[124, 125]. It may be noted that the solution of the SWE for the right electrode would be similar. Let's now write the SWE for the scattering region with Hamiltonian $H_{S}$ as 124, 125]

$$
\left[-\frac{\hbar^{2}}{2 m} \nabla^{2}+V(r)\right] \phi_{n k}(r)=E \phi_{n k}(r)
$$


The eigen states $\phi_{n k}(r)$ can be obtained using the asymptotic boundary condition that the solution of Equation 3.14 must match the eigen function of the Hamiltonian $H_{R}$ and $H_{L}$ as $x \rightarrow \pm \infty$ i.e. [124, 125]

$$
\lim _{x \rightarrow \mp \infty} \phi_{n k}(r)=\psi_{n k L, R}(r)
$$

In the active scattering region, there are the travelling waves coming from both left and right sides of the junction. Let's assume an electron with energy $E_{i}$ and initial state $\phi_{i k i}(r)$ at $x \rightarrow-\infty$ (left side of the active scattering region) is coming toward the active scattering region. Since the potential is complex in nature, it is not possible to tell exactly the state of an electron when it reaches the active scattering region. However, when this electron reaches deep in the right electrode $(x \rightarrow+\infty)$, its eigen state can be expressed as the linear combination of the eigen states of the Hamiltonian $H_{R}$ at the right electrode and is given by 124 , 125]

$$
\phi_{i k i}^{+}(r) \rightarrow \sum_{f=1}^{N_{R}} t_{i f} \psi_{f k f}(r), \quad x \rightarrow+\infty
$$

$t_{\text {if }}$ and $N_{R}$, in Equation 3.16, are the complex coefficients and the number of eigen channels in the right lead for a given energy; the plus $(+)$ sign in the eigen function $\phi_{i k i}^{+}$ is indicative of the fact that it stems from the past wave function $\psi_{i k i}$. It may be noted that the decaying wave solution of $H_{S}$ has negligible effect in the region $x \rightarrow+\infty$. 
In the deep left $(x \rightarrow-\infty)$, the eigen state of an electron is the superposition of the incident wave and back-scattered states from the junction and is given by [124, 125]

$$
\phi_{i k i}^{+}(r) \rightarrow \psi_{i k i}(r)+\sum_{f=1}^{N_{L}} r_{i f} \psi_{f k f}(r), \quad x \rightarrow-\infty
$$

The average current $I\left(E_{i}\right)$ corresponding to the state with energy $E_{i}$ can be obtained by the integration of the current density along the y-z plane as

$$
\begin{aligned}
I_{i}\left(E_{i}\right) & =e<\phi_{i k i}^{+}|\hat{j}| \phi_{i k i}^{+}> \\
& =\frac{e \hbar}{2 m i} \int_{-\infty}^{+\infty} d y \int_{-\infty}^{+\infty} d z\left[\left[\phi_{i k i}^{+}(r)\right]^{*} \frac{\partial \phi_{i k i}^{+}(r)}{\partial x}-\phi_{i k i}^{+}(r) \frac{\partial\left[\phi_{i k i}^{+}(r)\right]^{*}}{\partial x}\right] \\
& =\frac{e \hbar k_{i}}{m L_{x}} \\
& =\frac{e v_{i}\left(k_{i}\right)}{L_{x}}
\end{aligned}
$$

Deep inside the left lead $(x \rightarrow-\infty)$, the current can be expressed as [124, 125]

$$
I_{L}\left(E_{i}\right)=I_{i}\left(E_{i}\right)\left(1-\sum_{f=1}^{N_{L}} R_{i f}\left(E_{i}\right)\right)
$$

$N_{L}$ and $R_{i f}$, in Equation 3.19, are the number of channels in the left electrode and the reflection probability; the reflection probability is given by [124, 125]

$$
R_{i f}\left(E_{i}\right) \equiv\left|r_{i f}\right|^{2} \frac{I_{f}\left(E_{i}\right)}{I_{i}\left(E_{i}\right)}
$$

$I_{i}\left(E_{i}\right)$ is the current due to $\psi_{i k_{i}}$. 
Deep inside the right lead $(x \rightarrow+\infty)$, the current is [124, 125]

$$
I_{R}\left(E_{i}\right)=I_{i}\left(E_{i}\right) \sum_{f=1}^{N_{R}} T_{i f}\left(E_{i}\right)
$$

where $N_{L}$ and $T_{i f}$, in Equation 3.21 , are the number of channels in the right electrode and the transmission probability respectively. The transmission probability is given by

$$
T_{i f}\left(E_{i}\right) \equiv\left|t_{i f}\right|^{2} \frac{I_{f}\left(E_{i}\right)}{I_{i}\left(E_{i}\right)}
$$

We make an assumption that the current in the deep left and deep right electrodes must be identical (steady-state condition). In the non-equilibrium condition obtained by the application of the bias $\mathrm{V}$, the distribution of the electrons at the right electrode will be different from their distribution at the left electrode, implying that the chemical potential for the left and the right electrode will also be different. The motion of these electrons through eigen channels will give rise to currents. The total current can be expressed as the sum of all currents due to all available eigen channels (at all possible energies) assumed to be independent of each other [124, 125].

$$
I=2 e \int d E\left[\sum_{i=1}^{N_{L}} \sum_{f=1}^{N_{R}} f_{L}(E) D_{i}\left(E_{i}\right) I_{i}\left(E_{i}\right) T_{i f}\left(E_{i}\right)-\sum_{i=1}^{N_{R}} \sum_{f=1}^{N_{L}} f_{R}(E) D_{i}\left(E_{i}\right) I_{i}\left(E_{i}\right) T_{i f}\left(E_{i}\right)\right]
$$

The factor 2 in Equation 3.23 is due to the two possible spin directions; $f_{L, R}(E)$ gives the distribution of the electrons at the left and the right electrodes as discussed 
earlier. If the lead is a one-dimensional material, its density of states (DOS) can be expressed as

$$
\begin{aligned}
D_{i}\left(E_{i}\right) & =\frac{L_{x}}{2 \pi} \frac{d k_{i}}{d E_{i}} \\
& =\frac{L_{x}}{2 \pi \hbar v_{i}}
\end{aligned}
$$

The transmission coefficient $\left(\mathrm{T}_{\mathrm{RL}}(\mathrm{E})\right)$ from right to left can be expressed as the sum of transmission probabilities through all the eigen channels at the right and left electrodes [124, 125].

$$
T_{R L}(E)=\sum_{i=1}^{N_{R}} \sum_{f=1}^{N_{L}} T_{i f}(E), \quad R \rightarrow L
$$

Similarly, the transmission coefficient $\left(\mathrm{T}_{\mathrm{LR}}(\mathrm{E})\right)$ from left to right can be expressed as the sum of the transmission probabilities through all the eigen channels at the left and right electrodes i.e. [124, 125]

$$
T_{L R}(E)=\sum_{i=1}^{N_{L}} \sum_{f=1}^{N_{R}} T_{i f}(E), \quad L \rightarrow R
$$

The conservation of particle flux during carrier transport demands that the transmission coefficients in Equations 3.25 and 3.26 must be equal i.e.

$$
T_{L R}(E)=T_{R L}(E)=T(E)
$$


Using Equations 3.23 and 3.27 , the total current through a nanoscale junction can be expressed as 124,125$]$

$$
\begin{aligned}
I & =\frac{e}{\pi \hbar} \int d E\left[f_{L}(E)-f_{R}(E)\right] T(E) \\
& =\frac{2 e}{h} \int d E\left[f_{L}(E)-f_{R}(E)\right] T(E)
\end{aligned}
$$

\subsection{Single Particle Green's Function Formalism}

In the last section, we showed that the total current for ballistic transport through a nanoscale junction can be expressed in terms of the transmission function $T(E)$ using Landauer formalism of electron transport. Let's go beyond that and provide a way to calculate $T(E)$ using a single particle many-body Green's function approach in real space. Let $\mathrm{L}$ and $\mathrm{R}$ denote the left and right semi-infinite electrodes; $\mathrm{S}$ denotes the sample or central region. The semi-infinite electrode L is coupled to the central region $\mathrm{S}$ by the coupling potential $C_{L S}+C_{L S}^{\dagger}[125]$. Similarly, $\mathrm{R}$ is coupled to $\mathrm{S}$ by the

potential $C_{R S}+C_{R S}^{\dagger}[125]$. It may be noted that the two semi-infinite electrodes are far apart, suggesting that there is no direct coupling between them. Let's express the total Hamiltonian as the sum of Hamiltonians of the isolated semi-infinite electrodes $\left(H_{L}\right.$ and $\left.H_{R}\right)$, isolated sample or central region $\left(H_{S}\right)$, and the coupling potentials 
$\left(C_{L S}+C_{L S}^{\dagger}\right.$ and $\left.C_{S R}+C_{S R}^{\dagger}\right)[124,[125]$.

$$
\begin{aligned}
H & =H_{L}+H_{R}+H_{S}+C_{L S}+C_{L S}^{\dagger}+C_{S R}+C_{S R}^{\dagger} \\
& =H_{L}+H_{R}+H_{S}+C_{L S}+C_{S L}+C_{S R}+C_{R S}
\end{aligned}
$$

It may be noted that the Green's function associated with the isolated Hamiltonians are assumed to be known[125]. Here, our motive is to find the Green's function of the central region $\mathrm{S}$ in the presence of the coupling with the semi-infinite electrodes [125]. For this, we begin by writing the SWE for the nanoscale device in matrix form as 124,125$]$

$$
\left(\begin{array}{ccc}
H_{L} & C_{L S} & 0 \\
C_{L S}^{\dagger} & H_{S} & C_{S R}^{\dagger} \\
0 & C_{S R} & H_{R}
\end{array}\right)\left(\begin{array}{c}
\left|\Phi_{L}\right\rangle \\
\left|\Phi_{S}\right\rangle \\
\left|\Phi_{R}\right\rangle
\end{array}\right)=E\left(\begin{array}{c}
\left|\Phi_{L}\right\rangle \\
\left|\Phi_{S}\right\rangle \\
\left|\Phi_{R}\right\rangle
\end{array}\right)
$$

Here $\left|\Phi_{L}\right\rangle,\left|\Phi_{S}\right\rangle$, and $\left|\Phi_{R}\right\rangle$ are the single particle eigen functions of their respective Hamiltonians $H_{L}, H_{R}$, and $H_{S}$. E is the injection energy of the tunneling electrons. From Equation 3.30, we get three equations of the form [124, 125]

$$
\begin{gathered}
H_{L}\left|\Phi_{L}\right\rangle+C_{L S}\left|\Phi_{S}\right\rangle=E\left|\Phi_{L}\right\rangle \\
C_{L S}^{\dagger}\left|\Phi_{L}\right\rangle+H_{S}\left|\Phi_{S}\right\rangle+C_{S R}^{\dagger}\left|\Phi_{R}\right\rangle=E\left|\Phi_{S}\right\rangle \\
C_{S R}\left|\Phi_{S}\right\rangle+H_{R}\left|\Phi_{R}\right\rangle=E\left|\Phi_{R}\right\rangle
\end{gathered}
$$


Using Equations 3.31, 3.32, and 3.33, we arrive at the following two equations 124 , 125

$$
\begin{aligned}
& \left|\Phi_{L}\right\rangle=G_{L} C_{L S}\left|\Phi_{S}\right\rangle \\
& \left|\Phi_{R}\right\rangle=G_{R} C_{S R}\left|\Phi_{S}\right\rangle
\end{aligned}
$$

where $G_{L, R}$ is the Green's function of the left and the right electrodes and are given by

$$
G_{L, R}=\frac{1}{E-H_{L, R}}
$$

Using the expression of the Green's function in Equation 3.32, we get

$$
\begin{gathered}
C_{L S}^{\dagger} G_{L} C_{L S}\left|\Phi_{S}\right\rangle+H_{S}\left|\Phi_{S}\right\rangle+C_{S R}^{\dagger} G_{R} C_{S R}\left|\Phi_{S}\right\rangle=E\left|\Phi_{S}\right\rangle \\
\left(E-H_{S}-\Sigma_{L}-\Sigma_{R}\right)\left|\Phi_{S}\right\rangle=0
\end{gathered}
$$

Equation 3.37 suggests that we can define a Green's function as 124, 125]

$$
G(E)=\frac{1}{\left(E-H_{S}-\Sigma_{L}-\Sigma_{R}\right)}
$$

This is a retarded Green's function and is the response function to an impulse excitation at $\mathrm{t}=0$. In Equation 3.37. $\Sigma_{L}=C_{L S}^{\dagger} G_{L} C_{L S}$ and $\Sigma_{R}=C_{S R}^{\dagger} G_{R} C_{S R}$ are the self-energy functions for the left and the right electrodes respectively. The physical 
significance of these functions is that they allow the exchange of energy and electrons between the central scattering region and the semi-infinite electrodes. These non-Hermitian matrices broaden the electronic energy levels in the central scattering region; the imaginary parts of these matrices give broadening or the inverse lifetime of energy levels, suggesting the electrons have a finite lifetime in this region before they vanish into the electrodes. This means using a single-particle Green's function approach, we reduced the intricate problem of a nanoscale device that includes the semi-infinite electrodes and the central scattering region to a problem of active scattering region, which is open to both electrodes through the self-energy functions 124,125 . The broadening functions $\Gamma_{L, R}$ can be calculated from self-energy functions using [124, 125]

$$
\Gamma_{L, R}=i\left[\Sigma_{L, R}-\Sigma_{L, R}^{\dagger}\right]
$$

Consequently, the transmission function is defined, in terms of the broadening functions of the left electrode, right electrode, and the Green's function, as the sum of transmission probabilities over all eigen channels available in the central scattering region[124, 125].

$$
T(E)=\operatorname{Tr}\left[\Gamma_{L} G \Gamma_{R} G^{\dagger}\right]
$$

Physically, the transmission function in Equation 3.40 gives the probability of transmission of electrons from the source to the drain.

Next, we extend the theory to a real system under non-equilibrium condition. As 
stated earlier, such non-equilibrium condition can be achieved by perturbing the system with an electric field $\vec{\epsilon}$ along the axis of the nanowire. The Hamiltonian of the active scattering region in presence of such field can be written as the sum of Hamiltonians without electric field $(H(0))$ and the perturbation term $\left.\left(\vec{\epsilon} \cdot \sum_{i} r \overrightarrow{(i}\right)\right)$ as 124,125$]$

$$
H(E, \epsilon)=H(0)+\vec{\epsilon} \cdot \sum_{i} r \overrightarrow{(i)}
$$

$r(i)$ represents the position of the $i^{t h}$ electron. In this thesis, we have calculated the single electron energy levels in the active scattering region self-consistently using the finite cluster real space orbital based DFT as implemented in Gaussian[128] and then extracted the Hamiltonian $\left(H_{M}(E, \epsilon)\right)$ of the nanowire segment only from the Hamiltonian $H(E, \epsilon)$ of active scattering region. Subsequently, we have obtained the bias-dependent single particle Green's function of the molecular part (nanowire segment) from the Hamiltonian $H_{M}(E, \epsilon)$ using

$$
G_{M}(E, \epsilon)=\left[E \times S-H_{M}(\epsilon)-\Sigma_{L}(\epsilon)-\Sigma_{R}(\epsilon)\right]^{-1}
$$

In Equation 3.42, $\Sigma_{L}$ and $\Sigma_{R}$ are the bias-dependent self-energy functions for the left and the right electrodes; E and S are the injection energy of the tunneling electron and overlap matrix whose dimension is same as $H_{M}$. The self-energy functions $\Sigma_{L, R}$ can be obtained from the bias-dependent nanowire-lead coupling matrices $C_{L, R}$ and 
the Green's function $G_{p}$ for the leads using [124, 125]

$$
\Sigma_{L, R}=C_{L, R}^{\dagger} G_{p} C_{L, R}
$$

Since the leads on either side of the nanowire in our calculations are identical, $G_{p}$ is identical for both leads in our calculations. Now one may ask: how do we obtain the Green's function for the leads? It is important to note that in the wideband approximation, the Green's function $G_{p}$ can be obtained from the DOS per electron $(\eta(E))$ using the mathematical relation [131, 132

$$
G_{p}(E)=-i \pi \eta(E) \times I_{n}
$$

In this thesis, the DOS per electron $(\eta(E))$ has been obtained from the bulk DOS of the material of the lead $(\mathrm{Au})$ using a periodic DFT; the energy grid spacing of 0.001 $\mathrm{eV}$ is considered for calculating DOS. Such periodic calculations to obtain DOS also justify that electrodes in our transport calculations are semi-infinite. $I_{n}$, in Equation 3.44, is an identity matrix with dimension $\mathrm{n} \times \mathrm{n}$; $\mathrm{n}$ is the total number of Gaussian basis functions used to represent $\mathrm{Au}$ atoms. In our calculations, the Fermi energy of the bulk $\mathrm{Au}$ electrode has been matched to the Fermi energy of the active scattering region so that the device is under equilibrium. The equilibrium Fermi energy of the nanowire-lead junction (active scattering region) is its Highest Occupied Molecular Orbital (HOMO). In the spin-polarized cases (to be discussed in the next section), 
the HOMO for the $\alpha$ and $\beta$ electrons are different; the highest occupied energy level among the $\alpha$ and $\beta$ electrons is the HOMO of the system.

\subsection{Spin Transport in a Nanoscale Junction}

In previous sections, we derived relations to study the charge transport through the nanoscale junction. But, an electron, in addition to its charge, possesses an additional degree of freedom called spins. Therefore, in this section, we extend our theory to incorporate spin degrees of freedom and provide a way to calculate the spin-polarized Green's function that can then be used to obtain the spin-polarized transmission and current. To study the spin transport phenomena in a nanoscale junction, we need to make the device magnetic. There are two ways to do this. The first way is to place the magnetic channel between the two non-magnetic electrodes (eg. Au). The channel, if not magnetic, can be made magnetic by introducing a spin-functionality into the material via doping. If the channel becomes ferromagnetic after doping, the device can act as a spin filter. The second way is to place a non-magnetic channel between the two magnetic electrodes $(\mathrm{Ni}, \mathrm{Co}, \mathrm{Fe})$. Such a device is called a spin valve.

In this thesis, we have doped magnetic impurity in the non-magnetic Ge-core/Si-shell nanowire and placed a finite segment of this material in between the non-magnetic gold electrodes to form a nanoscale junction. Since the nanowire segment is magnetic 
and the electrodes are non-magnetic in our prototypical device, the Hamiltonian and the self-energy functions are spin-polarized; the Green's function for the leads is spinunpolarized. The spin-dependent Green's function for the molecular part (nanowire segment) is given by 133

$$
G_{M}^{\sigma}(E, \epsilon)=\left[E \times S-H_{M}^{\sigma}(\epsilon)-\Sigma_{L}^{\sigma}(\epsilon)-\Sigma_{R}^{\sigma}(\epsilon)\right]^{-1}
$$

$\sigma$ in Equation represents the spin states in $\pm z$ directions. The self-energy function for the spin polarized cases can obtained using $[133$

$$
\Sigma_{L, R}^{\sigma}=C_{L, R}^{\sigma \dagger} G_{p}^{\sigma} C_{L, R}^{\sigma}
$$

where, the Green's function of the lead for the spin polarized cases is obtained using 133

$$
G_{p}^{\sigma}=-i \pi \eta^{\sigma} \times I_{n}
$$

In Equations 3.47, $\mathrm{n}$ is the total number of Gaussian basis functions for $\mathrm{Au}$ atoms in the lead, In is an identity matrix, and $\eta^{\sigma}$ is the spin-polarized bulk DOS per electron for the spin up or spin down electrons. For non-magnetic leads (Au), the spin-up and spin-down DOS are identical. The spin-polarized current in the open junction is calculated using 133

$$
I^{\sigma}=\frac{e}{\hbar} \int_{\mu_{1}}^{\mu_{2}} T^{\sigma}(E, V)\left[f\left(E, \mu_{2}\right)-f\left(E, \mu_{1}\right)\right] d E
$$


$\sigma$ refers to $\alpha$ or $\beta$ electrons, $\mu_{1}$ and $\mu_{1}$ are the electrochemical potentials of the two contacts, which are determined self-consistently. $T^{\sigma}(E, V)$ is the multi-channel transmission function that represents the sum of the transmission probabilities over all the channels; $\mathrm{f}$ is the Fermi distribution function, and $\mathrm{V}$ is the applied potential. The total current was calculated as $I=I^{\alpha}+I^{\beta}$. It may be noted that under equilibrium conditions, the Fermi energy of the bulk Au electrode (obtained using the k-space approach) is matched to the Fermi energy (HOMO energy obtained using the realspace approach) of the active scattering region in our calculations. The equilibrium Fermi energy of the nanowire-lead junction (active scattering region) is its Highest Occupied Molecular Orbital (HOMO). In the spin-polarized cases (to be discussed in the next section), the HOMO for the $\alpha$ and $\beta$ electrons are different; the highest occupied energy level among the $\alpha$ and $\beta$ electrons is the HOMO of the system. The transmission function is given by $T^{\sigma}(E)=\operatorname{Tr}\left[\Gamma_{L} G^{\sigma} \Gamma_{R} G^{\sigma \dagger}\right]$, where $\Gamma_{L, R}=i\left[\Sigma_{L, R}^{\sigma}-\right.$ $\left.\Sigma_{L, R}^{\sigma \dagger}\right]$ is the broadening term that gives the inverse life time of an energy level. 


\section{Chapter 4}

\section{Mn-doped Ge/Si core/shell}

\section{nanowire}

\subsection{Introduction}

Since their inception[18], core-shell semiconductor nanowires, built out of group IV elements such as Ge and $\mathrm{Si}$ are the subject of immense interest[19, 37, 38, 39, 40, 41, 134, 135, 136, 137, 138, 139, 140, 141, 142, 143, 144. This level of interest in these nanostructures can be attributed to their multi-functional applications ranging from next-generation electronics [37, 40, 145] to biosensors [146] to photovoltaics [147, 148] to quantum computing devices [38, 39, 143]. For example, 
the Ge-core/Si-shell nanowires, which are the materials of choice due to its compatibility with the current Si-based technology, have been successfully synthesized in high yield[18, 19, 37] and reported to exhibit ballistic transport at a low bias with scattering mean free path of $\sim 500 \mathrm{~nm}[19,37$. Converting these low dimensional semiconductors to spin active structures would offer an additional opportunity for using them in spin-based electronics of the future 44, 45, 46, 47, 48. Intentional adding of a small number of magnetic impurities[149, 150] would be a viable path to implement spin functionality into such a system without destroying completely its semiconducting property. In fact, there have been numerous studies of Mn dopants in $\mathrm{Si}$ and Ge nanowires [49, 50, 51, 52, 53, 54, 55, 56], nano-columns[151], and nanotubes[152]. Depending upon the concentration of Mn, they have been reported to exhibit ferromagnetism at room temperature.

However, unlike these homogeneous nanowires, where the stabilization of the ferromagnetic phase at room temperature is a major challenge due to the substrate effect and often requires alloying, doping $\mathrm{Mn}$ into the core region of a Ge-Si core-shell heterostructure nanowire would offer significant advantages. Due to the valence band offset between the Ge and Si in a core-shell nanowire, spin carriers in Mn-doped coreshell structure can be guided through the spin active Ge core of the wires resulting in complete suppression of spin lifetime degradation due to scattering and recombination with the surface states. Furthermore, due to the confinement of carriers to the core region, we could limit the momentum dependent randomization of spins 
(spin dephasing) during spin transport-an important prerequisite in spintronics [45]. Mn-doped core-shell channel can also alleviate the conductivity mismatch challenge associated with the Schottky junction at the semiconductor nanowire/metal interface. Despite these advantages, until now, no efforts are made in understanding the role of Mn dopant on carrier transport in a Ge-core/Si-shell nanowire junction.

In this work, we used a predictive first-principles density functional theory (DFT) to investigate the electronic structure and magnetic properties of Mn-doped Ge-core/Sishell nanowire heterostructures. We limit ourselves to a low concentration of $\mathrm{Mn}$ dopants in the Ge core part of the core-shell structure due to its low solubility in semiconductors [153]. Our calculations reveal that the addition of Mn dopants transforms the semiconducting Ge-Si core-shell nanowire to a stable half-metallic ferromagnet. The energy band diagram yields a semiconducting behavior for one spin direction while the metallic behavior for the other. Inclusion of spin-orbit (SO) interaction is found to have minimal effect on the energy band structure; a maximum SO splitting of $\sim 24 \mathrm{meV}$ is obtained at the crossing points of majority and minority bands. Subsequently, a quantum transport approach [133 is used to calculate spin-polarized transmission in a prototypical Mn-doped Ge-core/Si-shell nanowire junction to assess its usage in a spin-filtering device. A spin-filter efficiency of $90.4 \%$ is found, further confirming the spin selective property of this material. 


\subsection{Computational Method}

We considered a Ge-core/Si-shell nanowire along the $\langle 110\rangle$ direction as it has been reported to be the preferred growth direction for a diameter of less than $20 \mathrm{~nm}[37$. Since Mn prefers the substitutional site in Ge [52, 154, 155, 156], we replaced one of the Ge in the core region of the unit cell by a Mn atom. In order to avoid the undesirable scattering of the carriers during transport, the surface dangling bonds in the Mn-doped Ge-core/Si-shell nanowire are passivated by hydrogen atoms. A supercell is constructed by placing a unit cell comprised of $47 \mathrm{Ge}, 80 \mathrm{Si}, 48 \mathrm{H}$, and $1 \mathrm{Mn}$ in a rectangular grid with the nanowire wall to wall distance (along $\mathrm{x}$ and $\mathrm{y}$-axis) greater than $11 \AA$ between the cells ensuring negligible interaction between the nanowire and its replica. The infinite nanowire is built by stacking up the supercell in the z-direction; the percentage of $\mathrm{Mn}$ atom in the 128-atoms (excluding hydrogen atoms) unit cell is $0.78 \%$. Subsequently, the nanowire geometry is optimized and the electronic structure and magnetic properties are calculated using the plane-wave basis function and the spin-polarized density functional method as implemented in the Vienna Ab initio Simulation Package (VASP)[157, 158]. The generalized gradient approximation (GGA) in the form of the Perdew-Burke-Ernzerhof (PBE) functional[105] is used to approximate the exchange-correlation potential. The Projector Augmented Wave (PAW) pseudopotential is used to model the valence-core interactions. During the geometry optimization, structural relaxations that include the strain effect due to Mn 
dopant and lattice mismatch between $\mathrm{Si}$ and Ge are carried out without symmetry constraint until the residual force on each atom reduces to $0.01 \mathrm{eV} / \AA$; the convergence criterion for total energy is set at $10^{-7} \mathrm{eV}$. The optimized unit cell structures of the Mn-doped Ge-core/Si-shell nanowire for different doping positions in the core (I, II, and III) and shell (IV) have been presented in Figure 4.1. The Monkhorst-

(a)

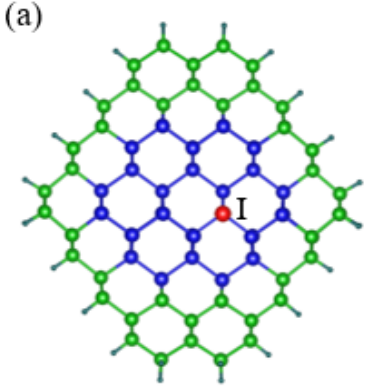

(c)

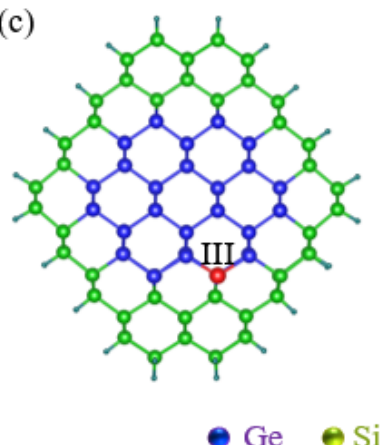

(b)

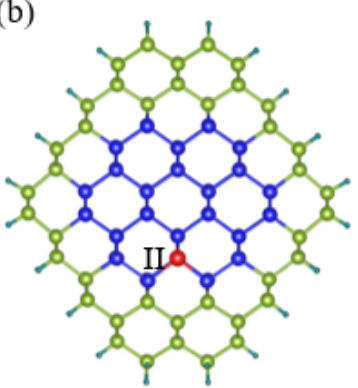

(d)

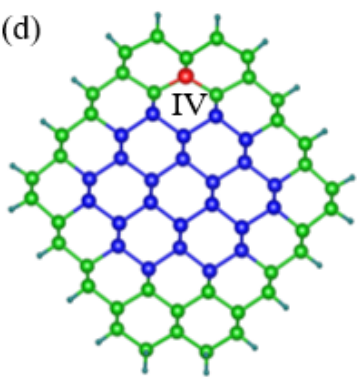

Figure 4.1: The top view of the optimized unit cell structures of Mn-doped Ge-core/Si-shell nanowire along the $\langle 110\rangle$ direction for different doping sites (I, II, III, and IV). The core diameter of the nanowire is $11.7 \AA$; the unsaturated surface states are passivated by H-atoms. (Reproduced from Nanoscale Adv. 2020, 2, 1843-1849 with permission from Royal Society of Chemistry.)

Pack (MP) k-point mesh of $1 \times 1 \times 7$ and the kinetic energy cut-off value of $400 \mathrm{eV}$ are used for these calculations. To correct the self-interaction error associated with the use of PBE functional, we have also used a hybrid functional (HSE06) [107, 159] that 
blends part of the Hartree-Fock exchange with the exchange and correlation potential from the PBE functional. A non-collinear spin-polarized calculation that includes spin-orbit interaction is also performed to measure the spin-orbit coupling induced splitting of energy bands. To examine the spin-filtering property of this material in a device configuration, we have constructed a prototypical Mn-doped Ge-core/Si-shell nanowire junction; a finite segment of the nanowire is sandwiched between two metallic gold electrodes with the electrode-electrode distance of $\sim 2.37 \mathrm{~nm}$. To avoid charge trapping at the lead-nanowire interface, we have passivated the unsaturated dangling states of the finite nanowire at the interface by H-atoms as done for the surface states. A real space orbital dependent spin unrestricted DFT approach as implemented in Gaussian [128] is used to construct the spin-polarized retarded Green's function $\left(G^{\sigma}\right)$ for the open junction [133] by dividing it into two parts: (a) an active scattering part consisting of the finite nanowire channel and 38 atoms from the gold lead and (b) an unperturbed gold lead that retains its bulk properties. The inclusion of gold atoms from the lead during the self-consistent calculation allows us to include explicitly the charging effects due to coupling with the semi-infinite electrodes. We used a posteriori hybrid B3LYP[128] exchange-correlation functional that partly corrects the self-interaction error for this calculation. This hybrid functional has been shown to give a much better description of transmission than pure functional[160]. In addition, a recent density of states analysis in transition metal compounds [161] has shown that the B3LYP results compare well with the results obtained from embedded dynamical 
mean-field theory. The convergence thresholds for total energy, root mean square and maximum density are set at $10^{-9}, 10^{-8}, 10^{-6}$ a. u. respectively. An all-electron 6-311g* Gaussian basis function [128] is used for Mn and H. For practical purposes, the $\mathrm{Ge}, \mathrm{Si}$, and $\mathrm{Au}$ atoms are represented by LANL2DZ effective core-potential basis set [128]. Subsequently, the transmission function for the majority and minority spin carriers is calculated as a function of injection energy using a spin-conserved tunneling approach[133] that does not take into account of the incoherent spin-flip scattering effect. The details of our method can be found in Chapter 3 of this thesis and in previous work 133$]$.

\subsection{Results and Discussions}

We begin by examining the energy of the Mn-doped Ge-core/Si-shell nanowire when a $\mathrm{Ge}$ or $\mathrm{Si}$ atom at various sites in the nanowire is replaced by a Mn atom (Figure 4.1). Our calculations reveal that the energy of the nanowire increases as we dope Mn away from the center of the core in the radial direction (Figure 4.2(a)). The energy barrier for $\mathrm{Mn}$ in going from the core position I to the shell position IV is $\sim 1.4 \mathrm{eV}$, suggesting the core positions are the preferred positions for Mn. The most energetically stable nanowire structure is illustrated in (Figure 4.1(a)). The total energy vs. lattice parameter curve (Figure 4.2(b) is calculated to determine the equilibrium lattice parameter $\mathbf{a}$ of the nanowire. The value of $\mathbf{a}$ is found to be $7.92 \AA$ 
in the doped nanowire, which is $0.01 \AA$ shorter than that in the undoped Ge-Si coreshell nanowire of a similar dimension.

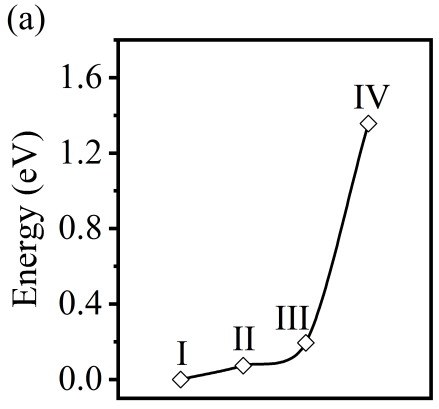

Mn-Position (b)

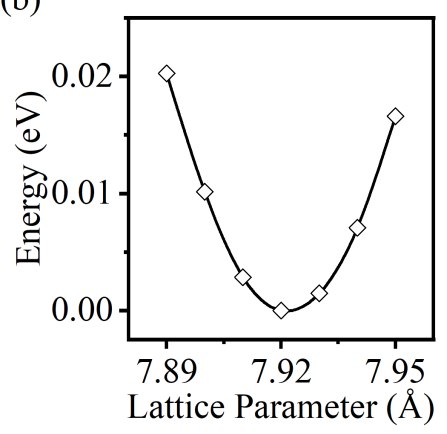

Figure 4.2: (a) Energy vs. Mn position in the nanowire; I, II, III, IV refer to the Mn positions.(b) Energy vs. Lattice parameter of the nanowire. The minimum energy is set to zero value in the energy scale for both (a) and (b). (Reproduced from Nanoscale Adv. 2020, 2, 1843-1849 with permission from Royal Society of Chemistry.)

The Mn and the nearest neighbor Ge bond distance $(2.42 \AA)$ is found to be $0.04 \AA$ shorter than the Ge-Ge bond distance $(2.46 \AA)$ of the undoped nanowire indicating a lateral bond strain of $\sim 1.6 \%$ upon substitutional doping of Mn. This is expected because we replaced the Ge with a larger electron cloud $(\mathrm{Z}=32)$ by the smaller $\mathrm{Mn}$ $(\mathrm{Z}=25)$. From bond angle analysis, we find the tetrahedral symmetry around Ge is distorted upon the substitution of Mn atom; the maximum angular deviation of 5.5\% is found at the Mn site.

Next, we comment on our calculated electronic band structure. Upon substitutional doping of $\mathrm{Mn}$, the strong exchange interaction arising from the unpaired d-electrons 

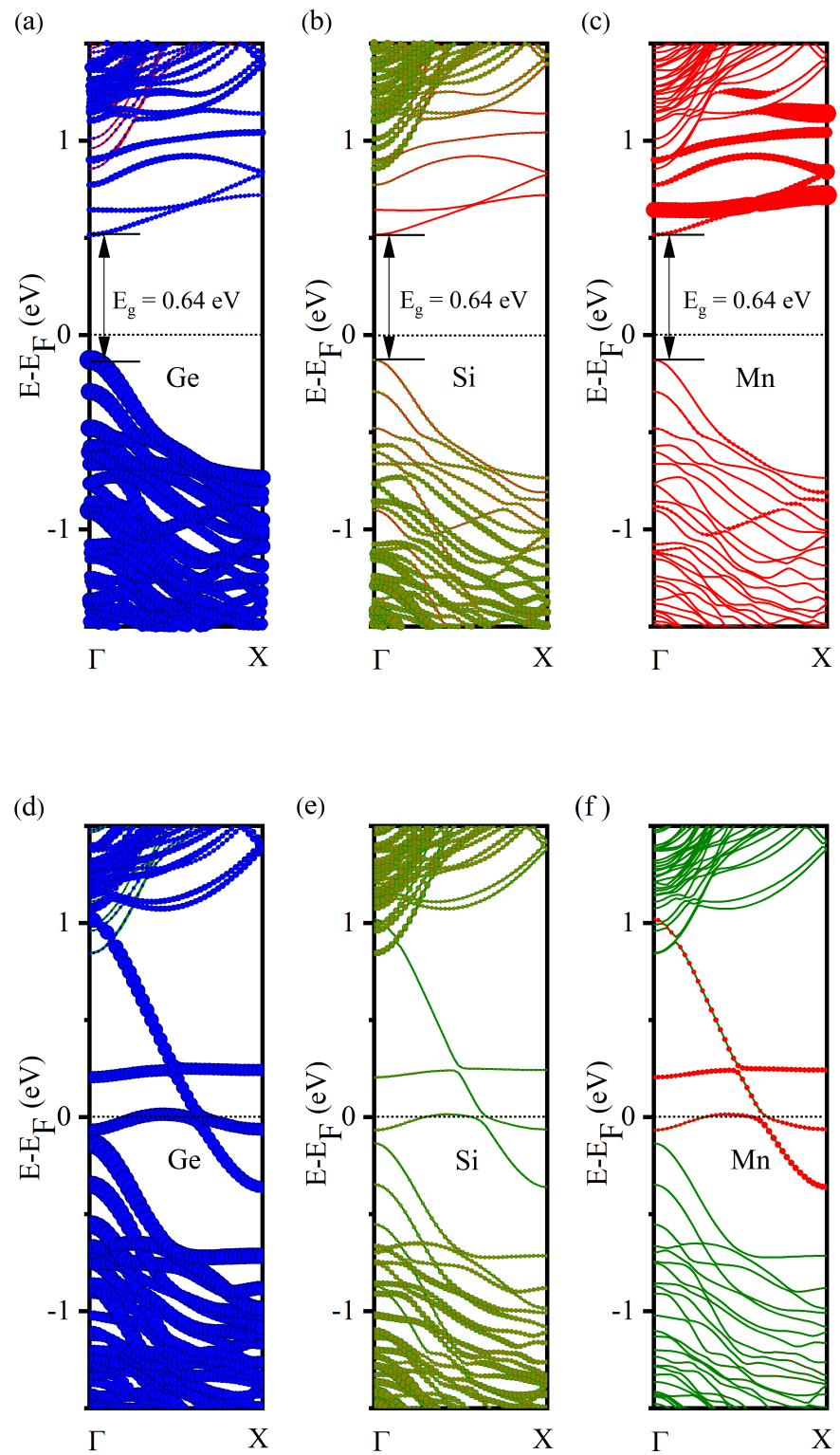

Figure 4.3: Atom decomposed electronic band structure (PBE) of Mndoped Ge-core/Si-shell nanowire in the (a-c) Minority-spin direction (d-f) Majority-spin direction; the circle represents the contribution of $\mathrm{Ge}, \mathrm{Si}$, and Mn atoms to energy bands. (Reproduced from Nanoscale Adv. 2020, 2, 1843-1849 with permission from Royal Society of Chemistry.)

of Mn splits the spin-degenerate energy bands of Ge-Core/Si-shell nanowire to the 
minority and majority spin bands (shown in Figure 4.3). A half-metallic feature is clearly noticeable in Figure 4.3. The minority spin electrons (Figures 4.3(a), 4.3(b), and $4.3(\mathrm{c}))$ exhibit a semiconducting behavior with an energy gap of $0.64 \mathrm{eV}$. The valence band maximum and the conduction band minimum are found at the same $\Gamma$ point confirming the direct nature of the bandgap as found for the undoped Gecore/Si-shell nanowire. The undoped nanowire of a similar diameter is reported to have a direct bandgap of $0.89 \mathrm{eV}[145$, 162]. The majority spin carriers (Figures $4.3(\mathrm{~d}), 4.3(\mathrm{e})$, and $4.3(\mathrm{f}))$, however, show a metallic behavior. Analysis of the atom decomposed band structure for minority spin direction reveals that the contributions to valence band at the $\Gamma$ point mainly come from the Ge atoms. However, in the case of the conduction band (CB), Mn and Ge contributions are comparable at the high symmetry $\Gamma$ point. As we move to the next higher energy band $(\mathrm{CB}+1)$, the contribution of the Mn dominates over Ge at the $\Gamma$ point. For the majority spin case, both Ge and Mn contribute to energy bands near the Fermi level. The magnetization is found to be localized around the $\mathrm{Mn}$ atom. The contribution to the magnetization mainly comes from the d orbital of $\mathrm{Mn}$ atoms and is found to be $\sim-3.18 \mu_{B}$ in the case of PBE functional. Though relatively small in magnitude $\left(\sim 0.08 \mu_{B}\right)$, the nearest neighbor Ge atoms are found to have magnetizations of opposite sign that comes from their $\mathrm{p}$ states. The local magnetic alignments in the vicinity of the Mn atom is shown in Figure 4.4(a), Similar local antiparallel magnetic alignments have been reported previously in Mn-doped systems [52, 150]. To understand the strength of exchange 
interaction between the Mn dopants, we double the unit cell size with the same Mn coverage and recalculated the energy (at zero temperature) for ferromagnetic (FM) and anti-ferromagnetic (AFM) coupling between the Mn atoms.

(a)

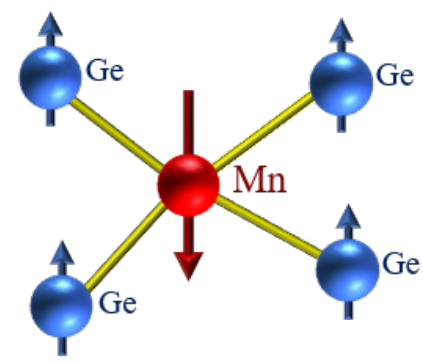

(b)

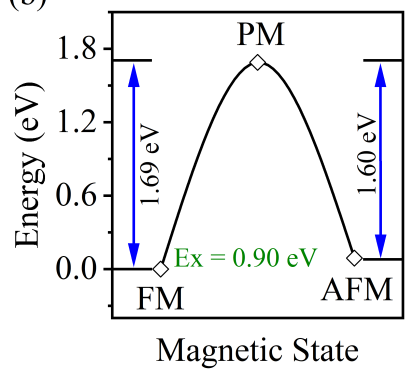

Figure 4.4: (a) Schematic showing the local alignment of magnetization of $\mathrm{Mn}$ and nearest Ge atoms in the nanowire. (b) Calculated energy for paramagnetic (PM), ferromagnetic (FM), and antiferromagnetic (AFM) configurations; the energy of the most stable FM state is set to zero. (Reproduced from Nanoscale Adv. 2020, 2, 1843-1849 with permission from Royal Society of Chemistry.)

The FM state is found to be lower in energy than the AFM state by $90.2 \mathrm{meV}$. A similar order for exchange-energy is reported in Mn-doped homogeneous nanowires[52]. For practical application at room temperature, however, we need to understand the thermodynamic stability of these magnetically ordered states. We calculated the metastable high entropy paramagnetic state (the expected transition point between FM and AFM state) to estimate the energy barrier (shown in Figure 4.4(b)). The energy barrier is found to be $1.69 \mathrm{eV}$, which is much higher than the room temperature (26 meV), suggesting that the FM ordering found here is stable at room temperature. 

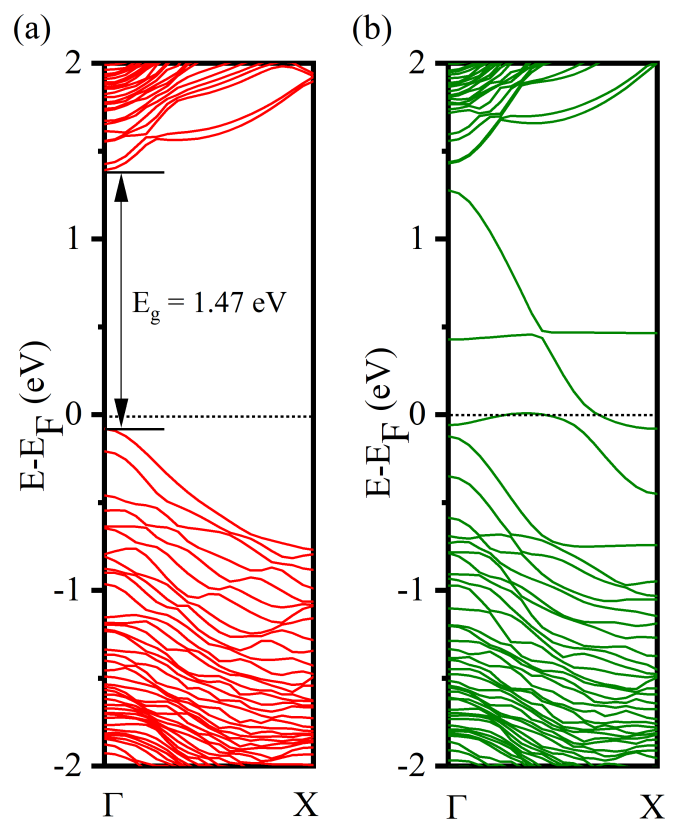

Figure 4.5: Electronic band structure (HSE06) of Mn-doped Ge-core/Sishell nanowire: (a) Minority-spin direction (b) Majority-spin direction. (Reproduced from Nanoscale Adv. 2020, 2, 1843-1849 with permission from Royal Society of Chemistry.)

To gauge the effect of self-interaction error associated with the use of PBE functional for the exchange-correlation energy and confirm the half-metallic property of our system, we have also performed the band structure calculation using a hybrid functional (HSE06). It has been reported that the use of HSE06 yields bandgaps closer to experimental values in group IV semiconductors[163]. Our results are presented in Figure 4.5. For the minority spin direction, a comparison of the band diagrams obtained using PBE functional (Figures 4.3(a), 4.3(b), and 4.3(c)) and HSE06 hybrid functional (Figure 4.5 ) reveals an increase in bandgap from $0.64 \mathrm{eV}$ to $1.47 \mathrm{eV}$ upon correcting 
(partly) the self-interaction error. However, the observed metallic behavior for the spin majority case (Figure $4.5 \mathrm{~b}$ ) is not affected by the self-interaction correction. The magnetic moment of the $\mathrm{Mn}$ is found to be $\sim-3.96 \mu_{B}$, which is higher in magnitude than that found with the use of PBE functional. The nearest neighbor Ge atoms are found to have oppositely aligned magnetic moments $\left(\sim 0.13 \mu_{B}\right)$ as observed for the PBE functional.

To gain a deeper insight into the origin of observed half-metallic behavior, we also calculated the spin-polarized atom decomposed and orbital decomposed density of states (DOS) for Mn-doped nanowire. The results obtained using the PBE functional are presented in Figures 4.6(a) and 4.6(b), In the minority spin case (Figure 4.6(a)), the energy gap is noticeable, which further confirms its semiconducting behavior; the Fermi level lies in the gap. The valence band is clearly dominated by Ge, which is also observed from the atom decomposed band structure in Figure 4.3. For the majority spin direction (Figure 4.6(b)), a finite DOS at the Fermi energy confirms its metallic character. Orbital decomposed DOS reveals that the hybridization of the d state (with some $\mathrm{p}$ contribution) of $\mathrm{Mn}$ and the $\mathrm{p}$ state of Ge is responsible for the metallic character, which is also evident from the atom decomposed band structure (Figure 4.3). The absence of energy states in the spin minority case and finite DOS in the spin majority case at the Fermi energy indicates $100 \%$ spin polarization in the Mn-doped Ge-core/Si-shell nanowire. 

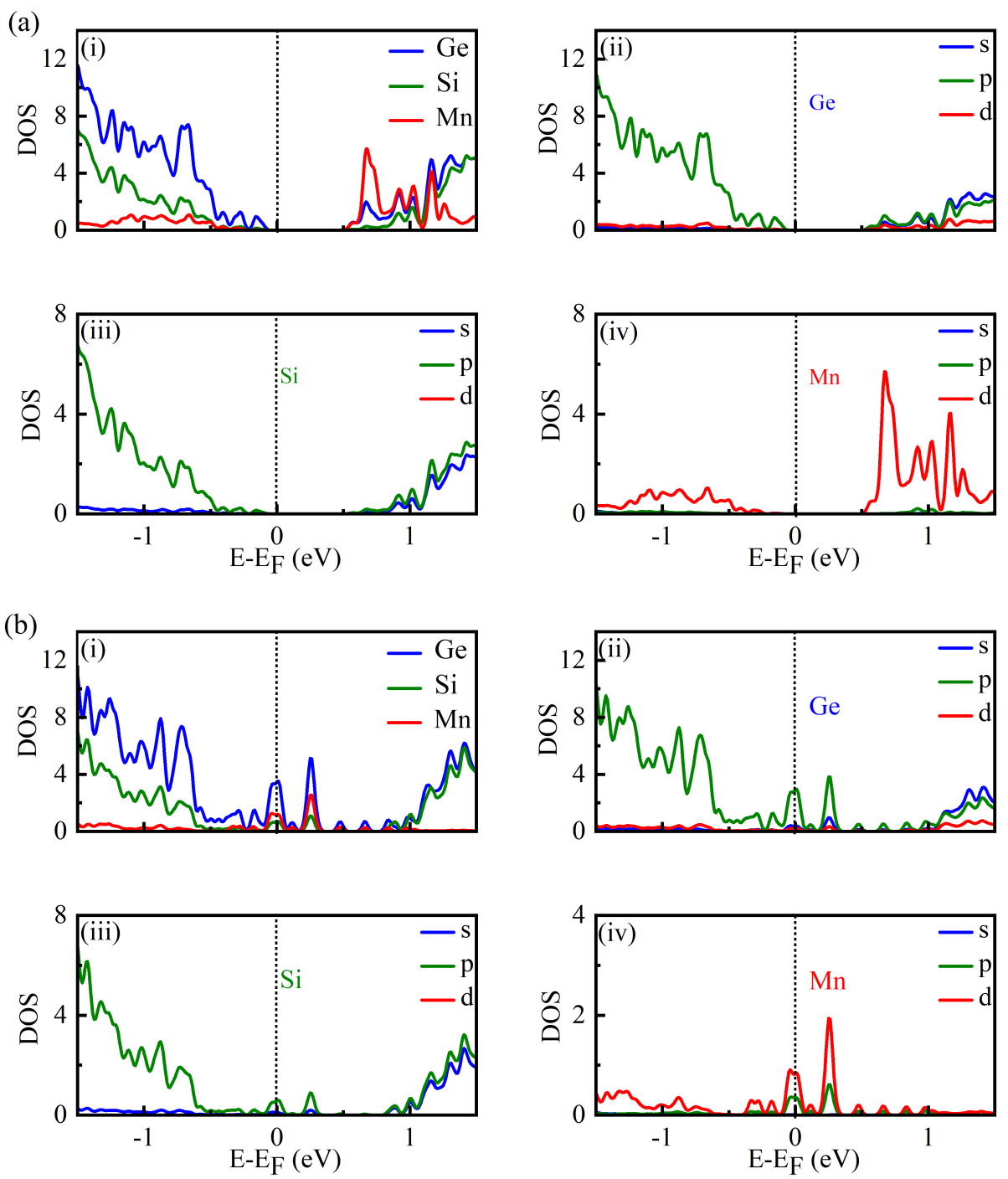

Figure 4.6: Atom and orbital decomposed density of states (DOS) of the Mn-doped Ge-core/Si-shell nanowire in the (a) Minority-spin direction (b) Majority-spin direction. (Reproduced from Nanoscale Adv. 2020, 2, 18431849 with permission from Royal Society of Chemistry.)

To further investigate the effect of spin-orbit interaction on energy bands of Mn-doped nanowire, we have performed the spin-unconstrained noncollinear DFT calculations that include the spin-orbit coupling (SOC) effect. Our results reveal that the inclusion of SOC does not alter the half-metallic property of our system, but as expected, it lifts 
the degeneracies at the crossings points of energy bands for the majority and minority spin carriers as shown in Figure 4.7. The maximum SO splitting at the band crossing
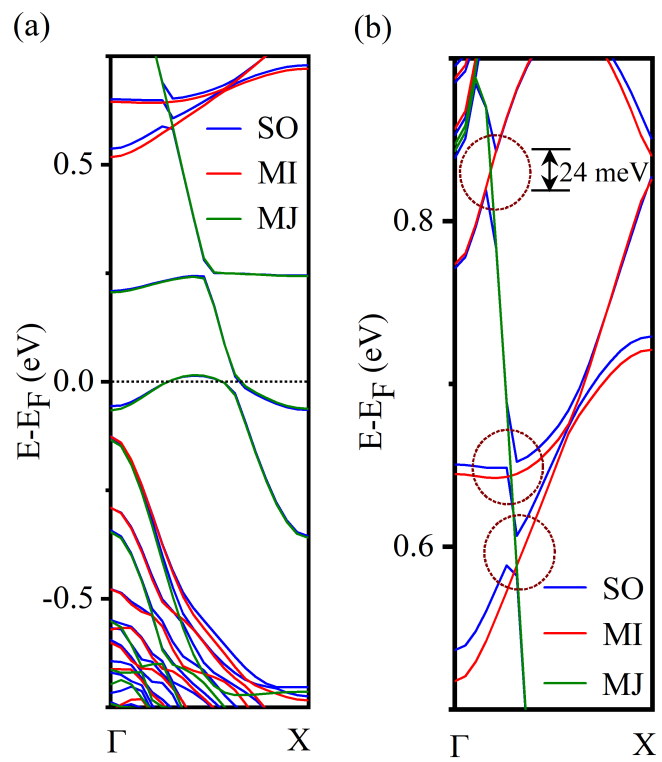

Figure 4.7: (a) The electronic band structure (PBE) of the Mn-doped Gecore/Si-shell nanowire with and without spin-orbit (SO) coupling; MJ and MI refer to the majority and minority spin directions. (b) Magnified version of (a) depicting SO splitting at the crossing points of MJ and MI bands. (Reproduced from Nanoscale Adv. 2020, 2, 1843-1849 with permission from Royal Society of Chemistry.)

is found to be $\sim 24 \mathrm{meV}$ (Figure 4.7(b)). However, no measurable shifts in energy levels in the vicinity of Fermi energy are observed due to spin-orbit coupling, which suggests that the spin coherence length can be much higher in this material. Thus far, we have focused only on the energetically most stable structure of Mn-doped nanowire and its properties. However, the doping or implantation of Mn into the Ge-core/Sishell nanowire is a non-equilibrium process. Hence, Mn may occupy other possible 

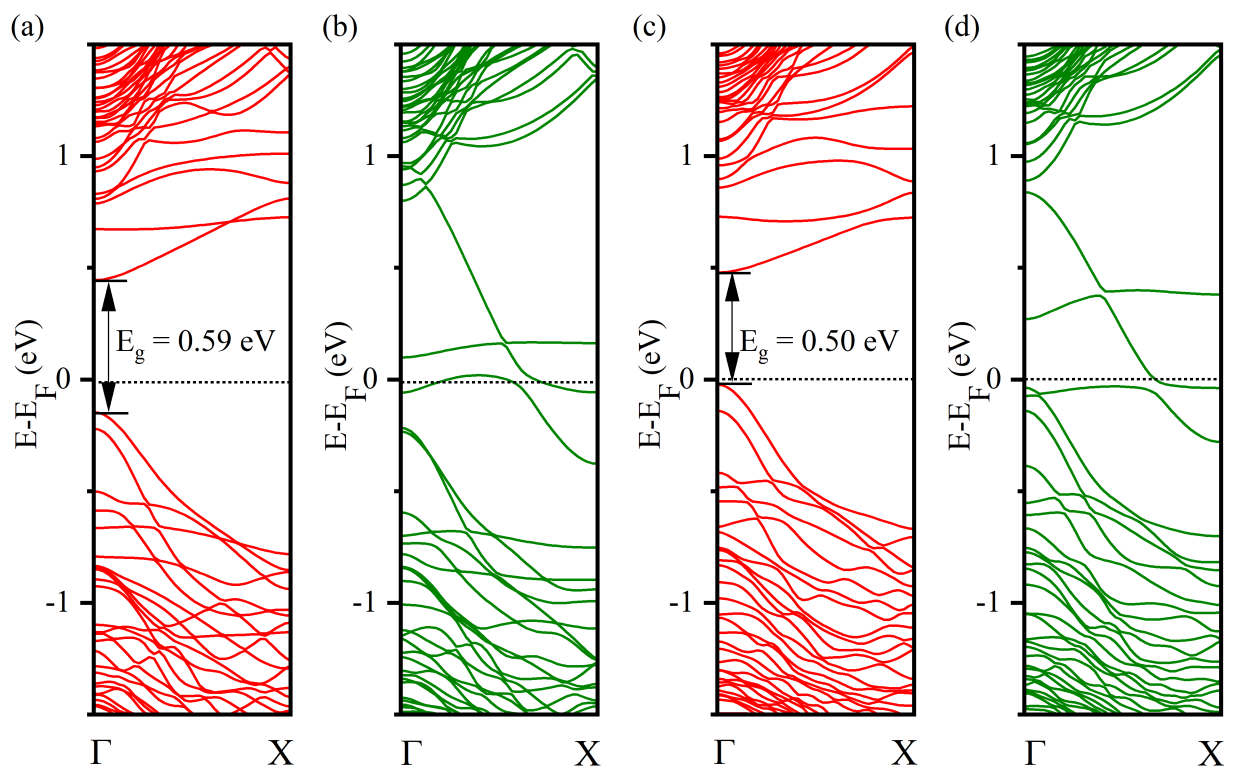

Figure 4.8: Electronic band structure (PBE) of Mn-doped Ge-core/Sishell nanowire in the (a) Minority-spin (b) Majority-spin direction when Mn atom is doped at site II. (c) and (d) represent the band structure in the Minority-spin and Majority-spin direction when Mn atom is doped at site III. (Reproduced from Nanoscale Adv. 2020, 2, 1843-1849 with permission from Royal Society of Chemistry.)

sites in the core as well as in the core and shell. To examine these possibilities, we have calculated the electronic band structure for Mn dopant at various substitutional sites (II and III in Figure 4.1). A half-metallic feature is clearly noticeable at both of these sites (Figure 4.8). We have also studied the case in which $\mathrm{Mn}$ is at the interstitial site (Figure4.9(a)). Though the half-metallic behavior is still observed, the minority spin electrons exhibit a semiconducting behavior with an indirect bandgap of 0.07 $\mathrm{eV}$ (Figure 4.9). Compared to site I, there is a significant decrease in bandgap for the minority spin direction in the case of interstitial doping. Now one may ask: Is 
(a)

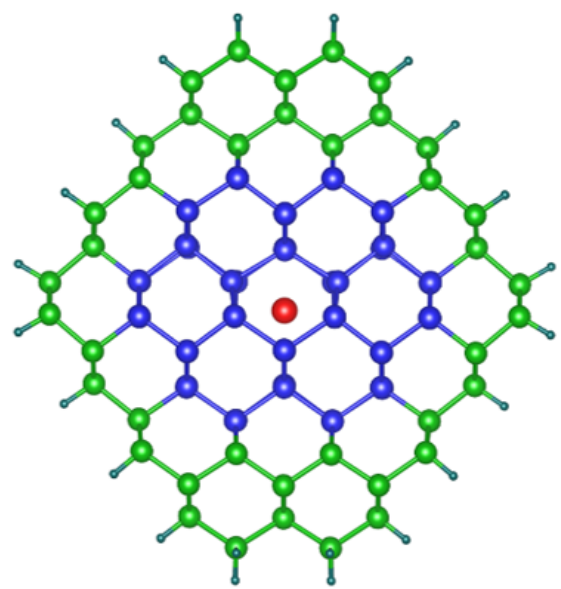

- $\mathrm{Ge} \odot \mathrm{Si} \odot \mathrm{Mn} \bullet \mathrm{H}$
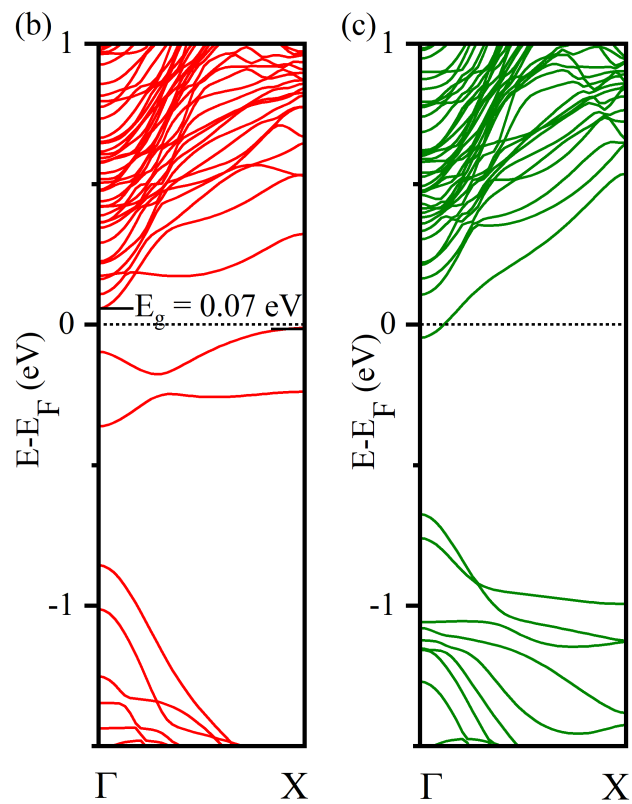

Figure 4.9: (a) The top view of the optimized Mn-doped Ge-core/Si-shell nanowire along the $\langle 110\rangle$ direction ( $\mathrm{Mn}$ at the interstitial site). The electronic band structure (PBE) of Mn-doped Ge-core/Si-shell nanowire in the (b) Minority-spin direction (c) Majority-spin direction when Mn is doped at the interstitial site; the half-metallic feature is noticeable. (Reproduced from Nanoscale Adv. 2020, 2, 1843-1849 with permission from Royal Society of Chemistry.) 
(a)

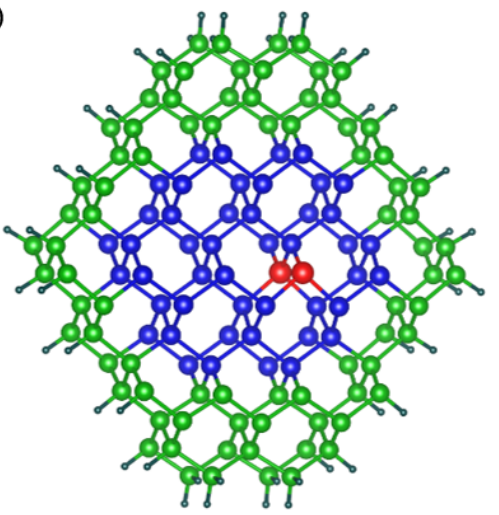

- $\mathrm{Ge}$ - $\mathrm{Si}$ (b)

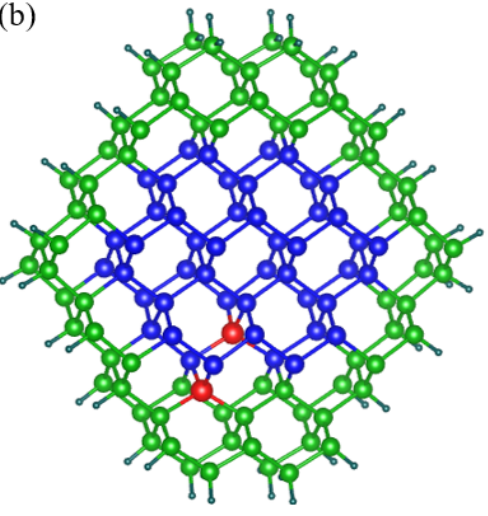

- $\mathrm{Mn}$ - $\mathrm{H}$ (c)

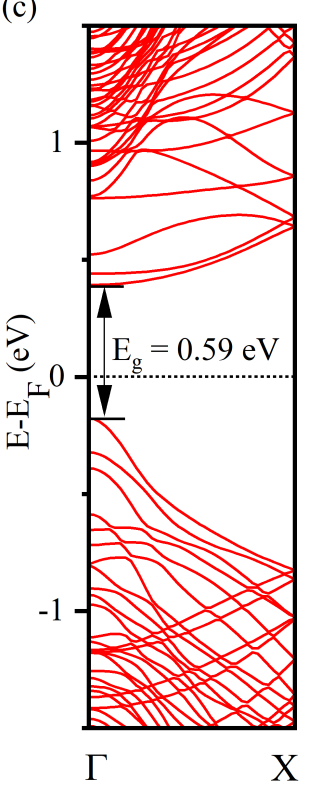

(d)

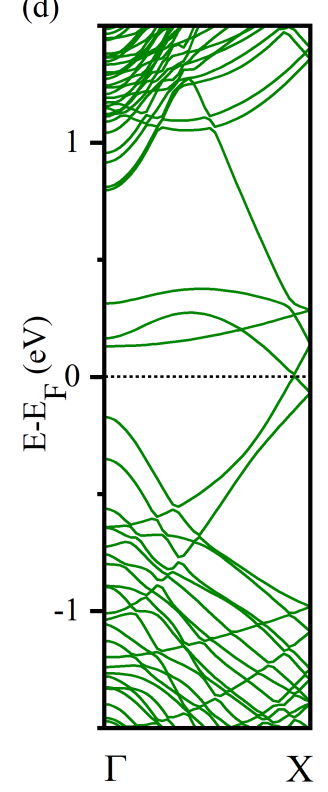

(e)

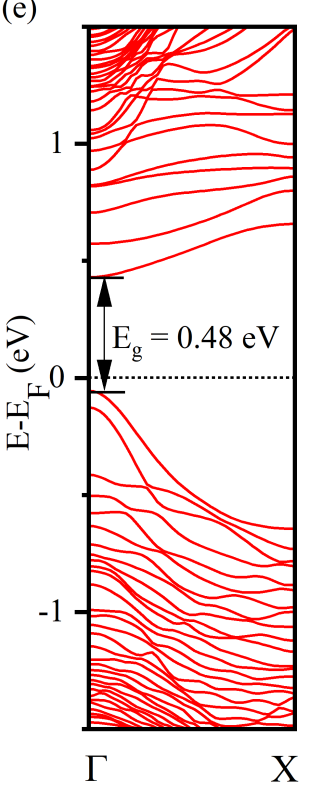

(f)

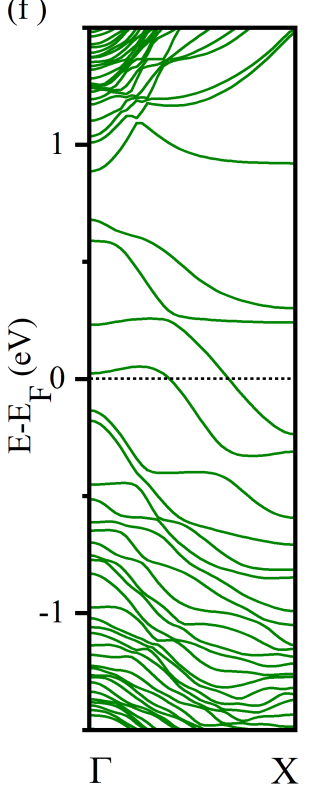

Figure 4.10: The optimized unit cell structure of Mn-doped Ge-core/Sishell nanowire when (a) both Mn atoms are doped in the core (b) one Mn atom is doped in the core and other in the shell. The electronic band structure in the (c) Minority-spin (d) Majority-spin direction when both Mn atoms are doped in core; (e) and (f) represent the energy band diagram in the Minority-spin and Majority-spin direction when one $\mathrm{Mn}$ atom is doped in core and other in the shell. (Reproduced from Nanoscale Adv. 2020, 2, 1843-1849 with permission from Royal Society of Chemistry.) 
the half-metallicity remains intact on increasing the concentration of Mn in the unit cell? To answer this, we increased the concentration of $\mathrm{Mn}$ in the unit cell from 0.78 $\%$ to $1.56 \%$. We will consider two cases: (a) both Mn atoms are doped in the core (Figure 4.10(a)) (b) one Mn atoms is doped in the core and other in the shell (Figure 4.10(b)). Our calculations show that a small increase in concentration of Mn atoms in the unit cell from $0.78 \%$ to $1.56 \%$ does not alter the half-metallic property (Figure 4.10).
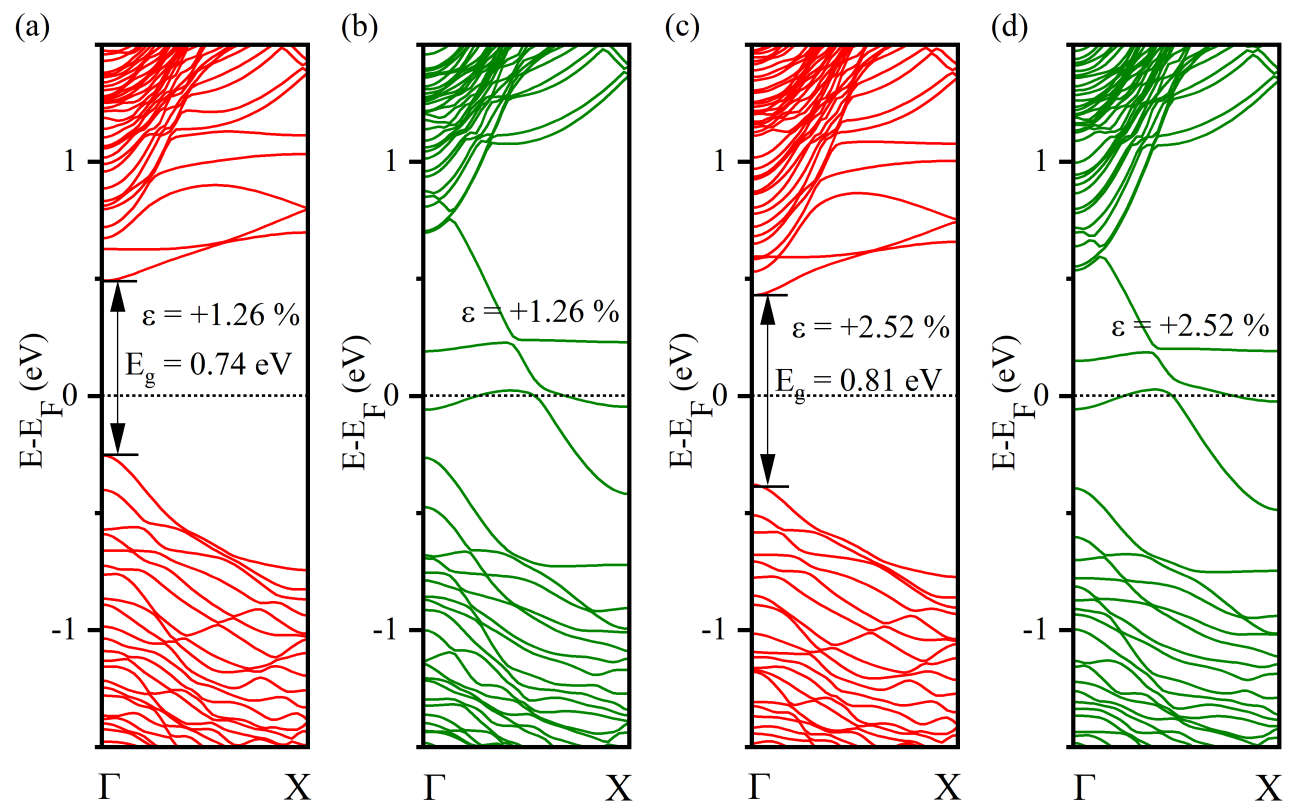

Figure 4.11: Electronic band structure (PBE) of Mn-doped Ge-core/Sishell nanowire (Mn at the site I) under tensile strain along the nanowire axis. (a) and (b) represent the band structure in the Minority-spin and Majority-spin direction under lateral strain of $+1.26 \%$; (c) and (d) represent the band structures in the Minority-spin and Majority-spin direction under lateral strain of $+2.52 \%$. (Reproduced from Nanoscale Adv. 2020, 2, 18431849 with permission from Royal Society of Chemistry.) 
To investigate the effect of the external strain, which may arise during synthesis of nanowire at finite temperature, we have also calculated the electronic band structure of the Mn-doped nanowire for both the tensile and compressive strain. To model the tensile or compressive strain, we have varied the lattice parameter a appropriately from its equilibrium value $(7.92 \AA)$ and allowed the atomic structure to relax without symmetry constraint until the residual force on each atom is less than $0.01 \mathrm{eV} / \AA$. As
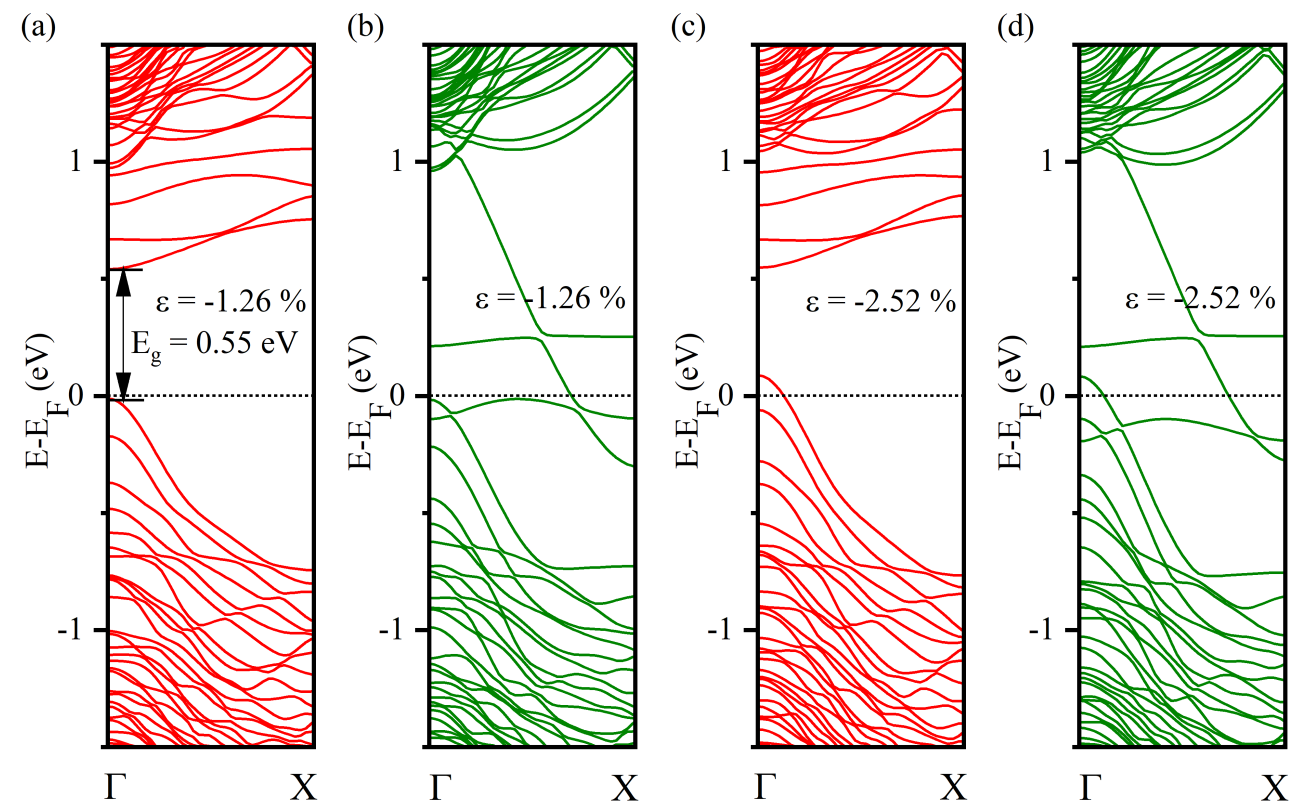

Figure 4.12: Electronic band structure (PBE) of Mn-doped Ge-core/Sishell nanowire ( $\mathrm{Mn}$ at site I) under compressive strain along the nanowire axis. (a) and (b) illustrate the band structures in the Minority-spin and Majority-spin direction under compressive strain of $-1.26 \%$; (c) and (d) represent the band structures in the Minority-spin and Majority-spin direction under compressive strain of $-2.52 \%$. (Reproduced from Nanoscale Adv. 2020, 2, 1843-1849 with permission from Royal Society of Chemistry.)

seen from the electronic band structure (Figure 4.11), the system is half-metallic in 
nature under tensile strain values of $+1.26 \%$ and $+2.52 \%$. The minority spin electrons display a semiconducting behavior in both the cases with a direct energy gap of 0.74 $\mathrm{eV}$ and $0.81 \mathrm{eV}$ respectively. The majority spin carriers, on the other hand, show a metallic characteristic. Our calculations reveal that the tensile strain along the nanowire axis is found to increase the bandgap in the minority spin direction. In the case of compressive strain (Figure 4.12), a half-metallic feature is clearly noticeable at the strain of $-1.26 \%$. The minority spin electrons exhibit a semiconducting behavior with a direct energy gap of $0.55 \mathrm{eV}$, whereas the majority spin carriers show a metallic behavior. However, for a large compressive strain of $-2.52 \%$, a semiconductor to metal phase transition in the minority spin direction is observed.

Next, to access the spin-filtering property of the Mn-doped Ge-Core/Si-shell nanowire, we have constructed a prototypical Mn-doped Ge/Core-Si-shell nanowire junction as shown in Figure 4.13(a). A spin conserved tunneling approach is used to calculate the transmission function for the majority $\left(\mathrm{T}_{\mathrm{MJ}}\right)$ and minority $\left(\mathrm{T}_{\mathrm{MI}}\right)$ spin carriers (Figure $4.13 \mathrm{~b}$ ); the transmission function is given by $T^{\sigma}(E)=\operatorname{Tr}\left[\Gamma_{L} G^{\sigma} \Gamma_{R} G^{\sigma \dagger}\right]$. Here, $G^{\sigma}$ is the spin polarized retarded Green's function given by $G^{\sigma}(E, \epsilon)=\left[E \times S_{N W}-\right.$ $\left.H_{N W}^{\sigma}(\epsilon)-\Sigma_{L}^{\sigma}(\epsilon)-\Sigma_{R}^{\sigma}(\epsilon)\right]^{-1}$, where $\mathrm{E}$ is the injection energy, $S_{N W}$ is the overlap matrix, $H_{N W}^{\sigma}(\epsilon)$ is the bias dependent Hamiltonian of the nanowire channel, and $\Sigma_{L}^{\sigma}(\epsilon)$ and $\Sigma_{R}^{\sigma}(\epsilon)$ are the bias-dependent spin-polarized self-energy functions for the left and the right electrodes that allow the nanowire channel to exchange spin-polarized electrons and energy with the semi-infinite electrodes. $\Gamma_{L, R}=i\left[\Sigma_{L, R}^{\sigma}-\Sigma_{L, R}^{\sigma \dagger}\right]$ is the broadening 
(a)

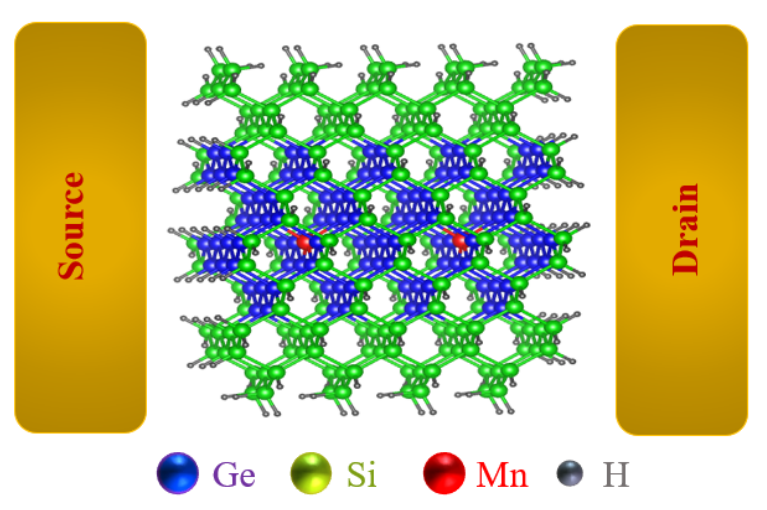

(b)

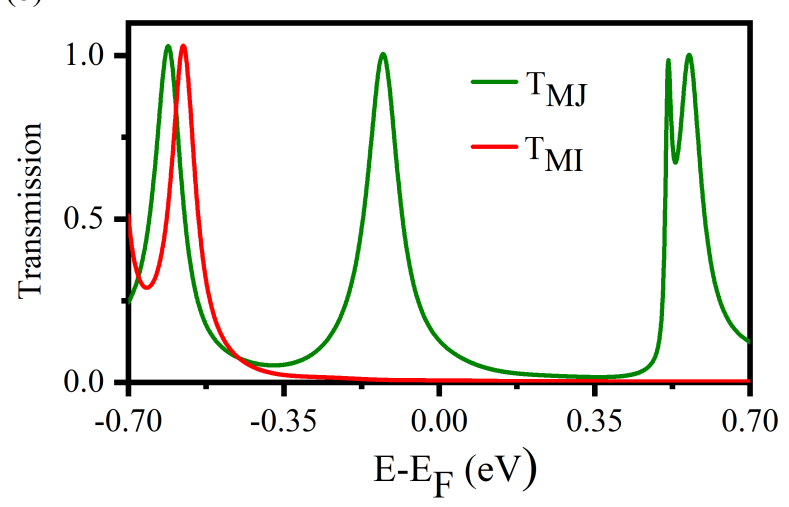

Figure 4.13: (a) A prototypical Mn-doped Ge-core/Si-shell nanowire spinfilter; the channel length is $\sim 2.37 \mathrm{~nm}$ (electrode-electrode distance). (b) Calculated spin-dependent transmission; $\mathrm{T}_{\mathrm{MJ}}$ and $\mathrm{T}_{\mathrm{MI}}$ refer to the transmission for the majority and minority spin carriers respectively. (Reproduced from Nanoscale Adv. 2020, 2, 1843-1849 with permission from Royal Society of Chemistry.)

term that gives the inverse life time of an energy level in the central scattering region.

Due to confinement of carries to the spin active Ge-core and observed weak spin-orbit interaction in the Mn-doped Ge-core/Si-shell nanowire, we assumed the scattering to be coherent and neglected the spin-flip scattering effect in our calculations. Figure $4.13 \mathrm{~b}$ shows that there are no transmission peaks found for the minority spin carries in the vicinity of the Fermi energy. However, a transmission peak appears close to the 
Fermi energy for the majority spin carriers. Analysis of orbital coefficients reveals that the p states of Ge and the d, as well as p, states of Mn that couple to the s and p states of gold electrode contribute to the transmission peak in the spin majority case. In the case of minority spins, the metal-induced broadening is responsible for an insignificant but a finite transmission value of $6.5 \times 10^{-3}$ at the Fermi energy. The $\mathrm{T}_{\mathrm{MJ}}$ at the Fermi energy is found to be $128.9 \times 10^{-3}$. To quantify the asymmetry in spin-dependent

transmission, we calculated the spin-filter efficiency, [164] $\eta=\frac{\mathrm{T}_{\mathrm{MJ}}\left(\mathrm{E}_{\mathrm{F}}\right)-\mathrm{T}_{\mathrm{MI}}\left(\mathrm{E}_{\mathrm{F}}\right)}{\mathrm{T}_{\mathrm{MJ}}\left(\mathrm{E}_{\mathrm{F}}\right)+\mathrm{T}_{\mathrm{MI}}\left(\mathrm{E}_{\mathrm{F}}\right)}$ using the transmission values for the majority and minority spin carries at the Fermi energy. The value for $\eta$ is found to be $90.4 \%$, which unambiguously confirms the spin-selective property of Mn-doped Ge-core/Si-shell nanowire channel. We expect the $\eta$-value to approach $100 \%$ with an increase in channel length of the nanowire as the transmission of the minority carrier with a semiconducting feature would fall exponentially [165] with an increase in the length of the channel. An increase of spin-filtering efficiency has been reported with the increase of channel length in other materials [164].

\subsection{Conclusions}

In summary, we predict that a small amount of Mn dopants in the core region of a GeCore/Si-Shell nanowire can transform the semiconducting Ge-Si core-shell nanowire to a half-metallic ferromagnet with $100 \%$ spin polarization at the Fermi energy. The 
ferromagnetic spin ordering is found to be stable at room temperature. The spinunconstrained non-collinear magnetic calculation that includes spin-orbit interaction reveals no measurable shift in energy levels in the vicinity of Fermi energy, which suggests that the spin-coherence length can be much larger in this material. The high spin-filter efficiency (>90\%) obtained using a quantum transport approach in a prototypical nanowire junction further confirms the spin-selective property of this material. We expect this new finding will generate experimental interest in this material for possible application in room temperature spintronics. 


\section{Chapter 5}

\section{Cr-doped Ge/Si core/shell}

\section{nanowire}

\section{$5.1 \quad$ Introduction}

Antiferromagnetic (AFM) materials are the ones that have magnetic moments alternating between the adjacent atomic sites or layers resulting in a zero-net magnetization. Due to this, it is prohibitively difficult to probe the AFM spin ordering in a material by an external magnetic field. However, the recent success in manipulating individual spin moments in an AFM material by electrical current [58] has opened up a new frontier in spintronics research [59, 60, 61, 62]. Further, antiferromagnets 
have been shown to exhibit fast spin-dynamics that lie in the THz range 63, 64, 65]. Furthermore, the absence of a stray magnetic field and the spin precession on a global scale in this material, which lead to a much weaker spin dephasing, are beneficial for their applications in spintronics [66, 166, 167, 168, 169, 170, 171, 172, 173]. At the same time, the semiconductors such as $\mathrm{Si}$ and Ge have been the driving force in revolutionizing the microelectronics industry for more than half a century. The successful synthesis[18, 19, 20, 21] of low dimensional structures out of these materials with reproducible electronic properties has further reaffirmed our hope of using these materials in future electronic circuits that demand smaller, lighter, and energy-efficient components. For example, the semiconductor GeCore/Si-Shell[18, 19, 37, 38, 39, 40, 41, 140] nanowire has been reported to exhibit superior transistor performance compared to the current state of the art MOSFETs[37]; programmable logic circuits based on these nanowire transistors are also demonstrated 43$]$.

Thus, a natural question one may ask: Can we harness the intriguing functionality of an antiferromagnet to this low-dimensional semiconductor? This may lead to a new class of materials with rich physics for potential applications in future generation antiferromagnetic semiconductor spintronics. The only viable approach to implementing antiferromagnetism in these materials without destroying their semiconducting behavior is via doping of magnetic impurities. In fact, several experimental techniques such as ion implantation and chemical vapor deposition have been successfully used 
to synthesize transition-metal-doped semiconductor nanowires [49, 50, 51, 54]. In addition, the core-shell configuration of these nanostructures provides an advantage of doping magnetic impurities into the core region of the wire, thus guiding the spins along the core during transport due to valence band offset $(\sim 0.5 \mathrm{eV})$ between the Ge-core and the Si-shell. Thereby, the spin-dephasing arising from the effect of the substrate can be completely eliminated during transport, consequently improving the performance of the spintronic device. Hence, the next question we need to answer: Is there any magnetic impurity that can transform this semiconductor nanowire to an AFM semiconductor? Because substitutional doping of Mn has been reported to transform this semiconducting material to a ferromagnetic half-metal[57], the possibility of AFM semiconducting behavior with Cr-dopant is explored in this study.

In this Letter, we present our calculations that reveal the Cr-doped Ge-Si core-shell nanowire as an AFM semiconductor. A first-principles spin-unrestricted density functional approach that explicitly considers the electron-electron interactions is used for our calculations. The stability of the AFM ordering between $\mathrm{Cr}$ atoms in the nanowire is ascribed to the superexchange interactions mediated by the $\mathrm{p}_{\mathrm{z}}$ orbitals of the bonded Ge atoms that lie in the proximity of Cr atoms, which is unique and outside of metal oxides [174]. The effect of spin-orbit (SO) interactions on the electronic band structure is found to be negligible, indicating the spins can be transported coherently in this material. Our spin-dependent quantum transport results indicate the electrical current in the doped $(\mathrm{Cr})$ nanowire to be significantly higher (more than one order) 
than that found in undoped nanowire for a threshold bias of $\geq 0.7 \mathrm{~V}$, suggesting it can be used as a switch.

\subsection{Computational Details}

We started with constructing a supercell of the Ge-core/Si-shell nanowire with 48 Ge, $80 \mathrm{Si}$, and $48 \mathrm{H}$ atoms along the $\langle 110\rangle$ direction as experimental studies have reported the $\langle 110\rangle$ as the favored growth direction for diameter less than $20 \mathrm{~nm}[37$. To ensure a negligible interaction between the nanowire and its periodic images, we created a supercell with a lattice parameter of $35 \AA$ in the $\mathrm{x}$ - and y-directions; there is a periodicity of $7.92 \AA$ along the z-direction. The unsaturated surface states were passivated by $\mathrm{H}$ atoms to avert the undesirable scattering of carriers during transport. Subsequently, we substituted Ge atoms at different sites with $\mathrm{Cr}$ atoms to model the doped nanowire as substitutional doping of $\mathrm{Cr}$ has been reported to be energetically favorable in Ge thin films[175]. The structure was then optimized without symmetry constraint until the residual force on each atom is less than 0.01 $\mathrm{eV} / \AA$ using spin-polarized density functional theory (DFT) with a plane-wave basis as implemented in VASP[157, 158]. The convergence criterion for the total energy was set at $10^{-7} \mathrm{eV}$. The kinetic energy cutoff value of $400 \mathrm{eV}$ was used for the planewave expansion. We used a dense Monkhorst-Pack 1x1x7 k-point grid to sample the Brillouin zone. The interactions between the valence electrons and the ionic core 
were described by the projector augmented wave method. The exchange-correlation potential was approximated by the Perdew-Burke-Ernzerhof (PBE) functional[105]. The self-interaction error associated with the PBE functional was partially corrected in our calculations by using a hybrid functional, HSE06 [107, 108], that includes a part of the exchange $(\sim 25 \%)$ from the Hartree-Fock and the remaining part $(\sim 75 \%)$ from the PBE functional; the correlation part is taken from the PBE functional. We further performed noncollinear magnetic calculations to study the effect of SO interactions on the energy bands of this material.

To study the quantum transport properties, we constructed a prototypical twoterminal Cr-doped Ge-core/Si-shell nanowire junction by sandwiching a segment of the nanowire between two gold electrodes; the electrode-electrode distance was $\sim$ 16.74 $\AA$. The open device consists of an active scattering part that includes a segment of the nanowire along with a finite number of gold atoms from the lead (seven on each side), and the semi-infinite part of the gold contact that retains its bulk properties. The spin-polarized retarded Green's function $\left(G^{\sigma}\right)$ for this open junction was then obtained by using a spin-unrestricted orbital-dependent posteriori DFT approach 133 . (implemented in Gaussian[128]) that uses Becke's three-parameter hybrid functional (B3LYP) 106, 128, for the exchange-correlation functional. This approach that partly corrects the self-interaction error due to explicit incorporation of a portion of HartreeFock exchange has been found to give a much better description of the transmission in nanoscale junction[160]. Furthermore, the density of states obtained using B3LYP 
functional in $3 \mathrm{~d}$ transition metal systems has been shown to agree well with that obtained from embedded dynamical mean-field theory (eDMFT)[161]. The manyelectron wave function in this approach is expressed by a single Slater determinant, in which the electrons are described by molecular orbitals constructed from atom centered Gaussian basis functions [128]. The Ge, Si, and Au atoms were represented by an effective core-potential double- $\zeta$ Gaussian basis set (LANL2DZ) that incorporates the scalar relativistic effect for $\mathrm{Au}$; $\mathrm{Cr}$ and $\mathrm{H}$ atoms were represented by an all-electron 6$311 \mathrm{~g}^{\star}$ Gaussian basis functions [128]. This explicit real space approach[133] allows us to partition the Kohn-Sham-Fock matrix in the active scattering region to construct $\left(G^{\sigma}\right)$

$$
G^{\sigma}(E, \epsilon)=\left[E \times S_{N W}-H_{N W}^{\sigma}(\epsilon)-\Sigma_{l}^{\sigma}(\epsilon)-\Sigma_{r}^{\sigma}(\epsilon)\right]^{-1}
$$

$\mathrm{E}$ is the injection energy, $S_{N W}$ is the overlap matrix, $H_{N W}^{\sigma}(\epsilon)$ is the bias dependent Hamiltonian of the nanowire channel; $\Sigma_{l}^{\sigma}(\epsilon)$ and $\Sigma_{r}^{\sigma}(\epsilon)$ are the bias-dependent spinpolarized self-energy functions for the left and the right electrodes that allow the nanowire channel to exchange spin-polarized electrons and energy with the semiinfinite electrodes. An electric dipole interaction term $\left(\overrightarrow{\epsilon_{d}} \cdot \sum_{i} \overrightarrow{\mathbf{r}_{i}}\right)$ was included in the Hamiltonian of the active scattering region during self-consistent electronic structure calculations to take into account of the bias effect[133]. The spin-polarized current in the open junction was calculated as 133]

$$
I^{\sigma}=\frac{e}{\hbar} \int_{\mu_{1}}^{\mu_{2}} T^{\sigma}(E, V)\left[f\left(E, \mu_{2}\right)-f\left(E, \mu_{1}\right)\right] d E
$$


$\sigma$ refers to $\alpha$ or $\beta$ electrons, $\mu_{1}$ and $\mu_{1}$ are the electrochemical potentials of the two contacts, which are determined self-consistently. $T^{\sigma}(E, V)$ is the multi-channel transmission function that represents the sum of the transmission probabilities over all the channels; $\mathrm{f}$ is the Fermi distribution function, and $\mathrm{V}$ is the applied potential. The total current was calculated as $I=I^{\alpha}+I^{\beta}$. It may be noted that under equilibrium condition, the Fermi energy of the bulk Au electrode (obtained using the k-space approach) is matched to the Fermi energy (HOMO energy obtained using the real space approach) of the active scattering region in our calculations. The detailed procedure for calculating spin-dependent current can be found in Chapter 3 of this thesis and in earlier work[133].

\subsection{Results and Discussions}

Because we do not know a priori the most energetically favorable substitutional sites for the Cr atoms in the Ge-core/Si-shell nanowire, we calculated the energy for various possible dopant configurations as shown in Figure 5.1. The configuration I (the unit cell structure is shown in Figure 5.1(a)) was found to be the most stable structure; the energy of the nanowire corresponding to different dopant sites is illustrated in Figure 5.2(a). Figure 5.2(b) depicts the variation of the total energy with the lattice parameter for configuration I; the equilibrium lattice parameter is found to be 7.92 A. For the rest of the paper, we will focus on the electronic, magnetic, and transport 


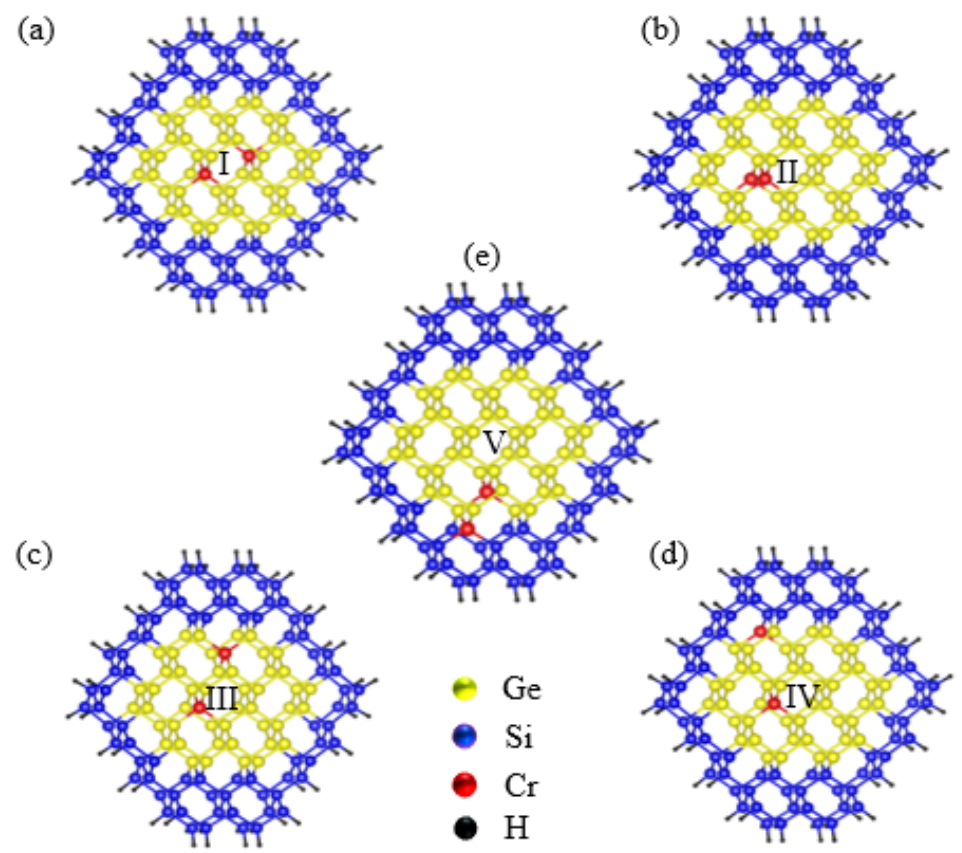

Figure 5.1: The optimized unit cell structure of the Cr-doped Ge-core/Sishell nanowire along the $\langle 110\rangle$ direction for various dopant configurations represented by I, II, III, IV, and V; the unsaturated surface states are passivated by H-atoms. (Reprinted with permission from Nano Lett. 2021, 21(4), 1856-1862. Copyright (2021) American Chemical Society.)

properties of the nanowire for the most stable structure, I. The bond-length analysis of the optimized structures in doped and undoped configuration indicates a maximum intrinsic bond strain of $\sim-2.02 \%$ at the substitutional site of the Cr; Ge-Ge bond length was $\sim 2.47 \AA$ in the undoped case as compared to a smaller Cr-Ge bond length of $\sim 2.42 \AA$ in the doped nanowire. The tetrahedral geometry of the Ge is distorted because of the substitutional doping of $\mathrm{Cr}$; a maximum angular strain of $\sim 1.52 \%$ was noted (the Ge-Ge-Ge bond angle in the undoped case was $\sim 109.83^{0}$ and upon doping Ge-Cr-Ge angle becomes $\sim 111.50^{\circ}$ at the corresponding site). Next, we discuss the 
(a)

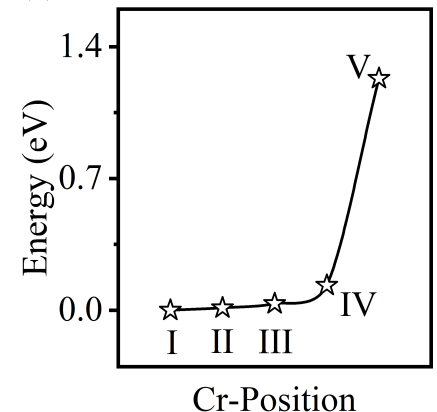

(b)

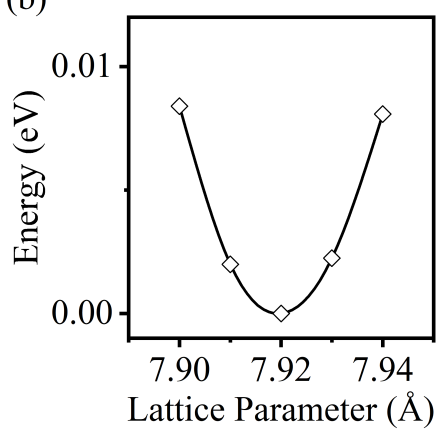

(c)

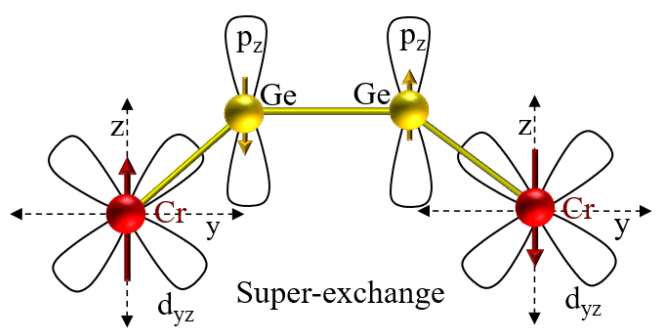

Figure 5.2: (a) Energy (relative to minimum energy configuration I) vs. Cr-configurations in the nanowire; the various configurations of the two Cratoms in the nanowire is represented by indices I, II, III, IV, and V (see Figure 5.1). (b) Energy vs. lattice parameter of the nanowire for configuration I. (c) Schematic showing the superexchange interaction mediated by the $p_{z}$ orbitals of the Ge that dictates the AFM spin ordering between $\mathrm{Cr}$ ( $d_{y z}$-orbitals). (Reprinted with permission from Nano Lett. 2021, 21(4), 1856-1862. Copyright (2021) American Chemical Society.)

magnetic properties of the Cr-doped Ge-core/Si-shell nanowire obtained using the PBE functional. Our calculations reveal that the $\mathrm{Cr}$ atoms in the doped nanowire prefers the antiparallel alignment of the magnetic moments at the Cr sites over the parallel alignment; the exchange energy $(\mathrm{Ex}=\mathrm{EAFM}-\mathrm{EFM})$ is $-10 \mathrm{meV}$. The electronic structure reveals that the localized d-orbital of $\mathrm{Cr}$ atoms contribute to the magnetization. The magnetization on the adjacent $\mathrm{Cr}$ atoms is found to be \pm 2.59 
$\mu_{B}$. A similar value for the magnetization was reported at the $\mathrm{Cr}$ sites in the $\mathrm{Cr}$ doped semiconductor GaN nanowire[150]. The Ge atoms that are bonded to the $\mathrm{Cr}$ atoms in our case are found to be weakly magnetized $\left( \pm 0.08 \mu_{B}\right)$ due to the magnetic proximity effect; the p states contribute to the magnetization at the Ge sites as shown in Figure 5.2(c). It should be noted that in the case of Cr-doped GaN nanowire, FM ordering was reported to be the stable configuration arising from the double exchange interaction. In the case of the Mn-doped Ge-core/Si-shell nanowire, which is a halfmetal, the FM spin ordering is found to be more favorable with an exchange energy of $\sim 90 \mathrm{meV}$, and a majority spin carriers exhibit a metallic behavior with a finite density of states at the Fermi energy[57]. This suggests that the exchange interaction that stabilizes the FM ordering between Mn atoms in the Mn-doped nanowire is mediated by the itinerant electrons (majority spin carries in the metallic channel) at the Fermi energy. However, in Cr-doped Ge-core/Si-shell nanowire, which is semiconducting (as discussed in the next paragraph), we find the AFM coupling between the localized $d_{y z}$ orbitals of the $\mathrm{Cr}$ atoms is found to be mediated by the $p_{z}$ orbitals of the bonded Ge atoms as shown in Figure 5.2(c), suggesting that the superexchange mechanism is responsible for the stability of the AFM ordering.

To gain a deeper insight, we calculated the spin-polarized atom projected electronic band structure; the results are presented in Figure 5.3. From Figure 5.3, one can notice that this material exhibits a semiconducting behavior with an indirect bandgap of $0.32 \mathrm{eV}$; the valence band maximum (VBM) is found at the $\Gamma$ point and the 

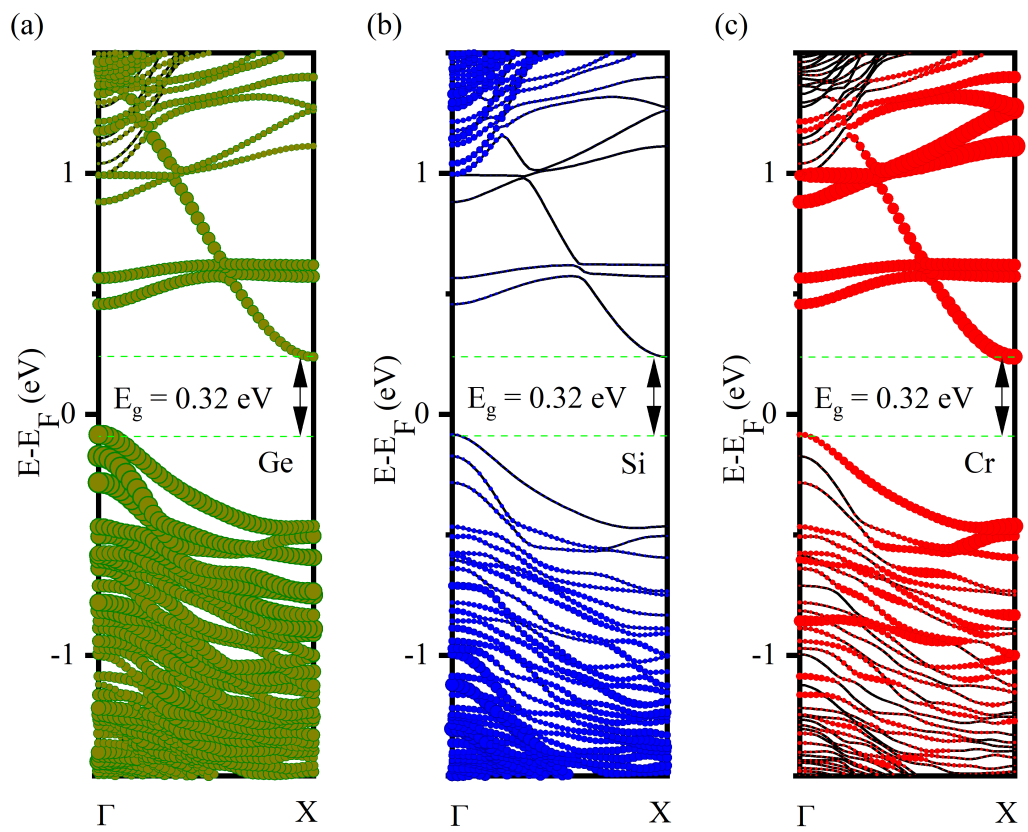

Figure 5.3: (a-c) Atom projected electronic band structure (PBE) of Crdoped Ge-core/Si-shell nanowire (configuration I) for the spin-up direction. The circle represents the contribution of the individual $\mathrm{Ge}, \mathrm{Si}$, and $\mathrm{Cr}$ atoms to energy bands. The larger the diameter of the circle, the larger the contribution. The energy band diagrams for the spin-down electrons are not shown as the energy levels for spin-up and spin-down directions are degenerate for this AFM system. (Reprinted with permission from Nano Lett. 2021, 21(4), 1856-1862. Copyright (2021) American Chemical Society.)

conduction band minimum $(\mathrm{CBM})$ is found at the $\mathrm{X}$ point. It is worthwhile to note that the undoped Ge-core/Si-shell nanowire is a direct bandgap semiconductor. A close examination further reveals that the contribution to VBM and CBM primarily comes from the Ge and Cr atoms as expected. Si atoms do not contribute to the bands near the Fermi level; they begin to contribute for bands with energy of about $-0.71 \mathrm{eV}$ and lower at the $\Gamma$ point, indicating a large valence band offset (VBO) of $\sim 0.63 \mathrm{eV}$ between the core $(\mathrm{Ge}$ and $\mathrm{Cr}$ ) and shell $(\mathrm{Si})$. This result further confirms 
that the significant VBO observed in the Ge-core/Si-shell nanowire remains intact upon substitutional doping of $\mathrm{Cr}$, suggesting spin carriers could be guided either through the core or the shell part of this nanowire channel during transport. The results obtained from the atom projected electronic band structure calculations are in agreement with that obtained from the density of states (DOS) calculations in Figure 5.4. DOS calculations further reveal that the $\mathrm{p}$ orbitals of Ge and d orbitals of $\mathrm{Cr}$ contribute mostly to the VB and CB near the Fermi level.
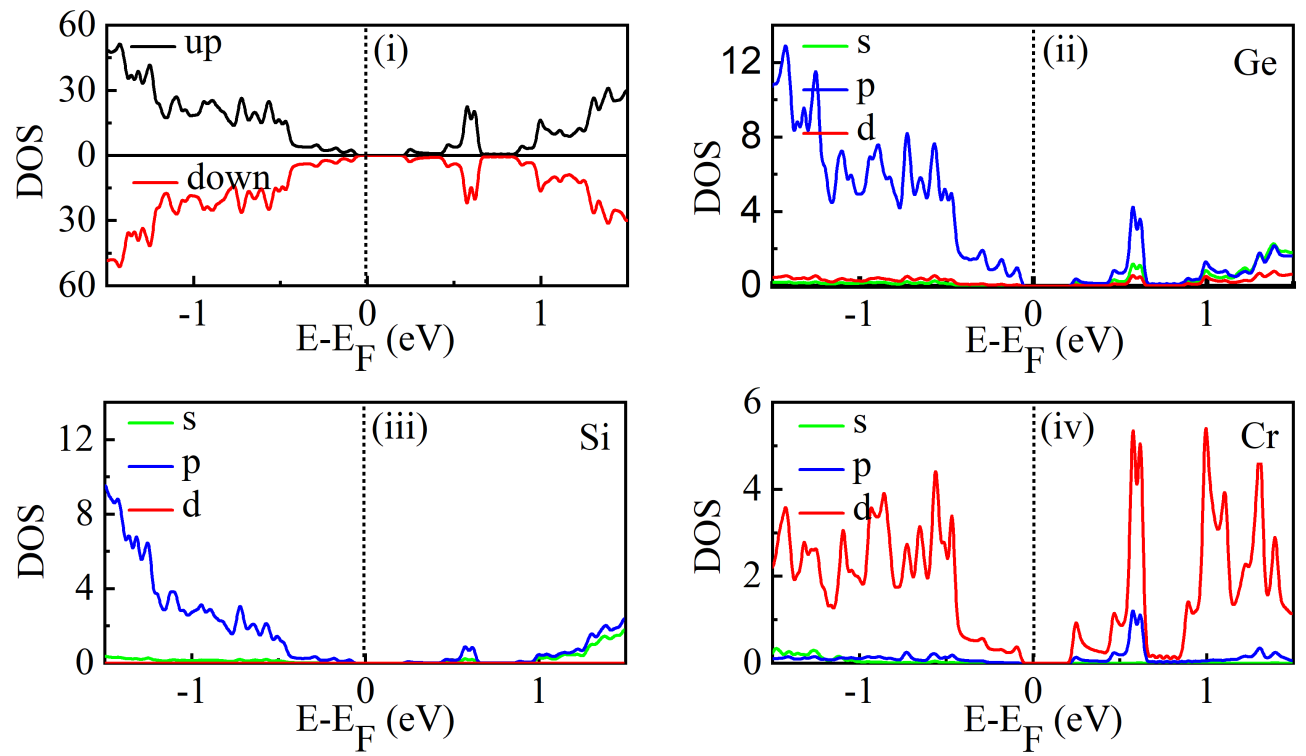

Figure 5.4: Total and orbital decomposed density of states (DOS) of Crdoped Ge-core/Si-shell nanowire (configuration I) (i) total DOS in spin up and spin down directions (ii-iv) orbital decomposed DOS for the Ge, Si, and $\mathrm{Cr}$ atoms respectively; the orbital decomposed DOS for the spin-down electrons are not shown as the energy levels for spin-up and spin-down directions are degenerate for this antiferromagnetic (AFM) system. (Reprinted with permission from Nano Lett. 2021, 21(4), 1856-1862. Copyright (2021) American Chemical Society.) 

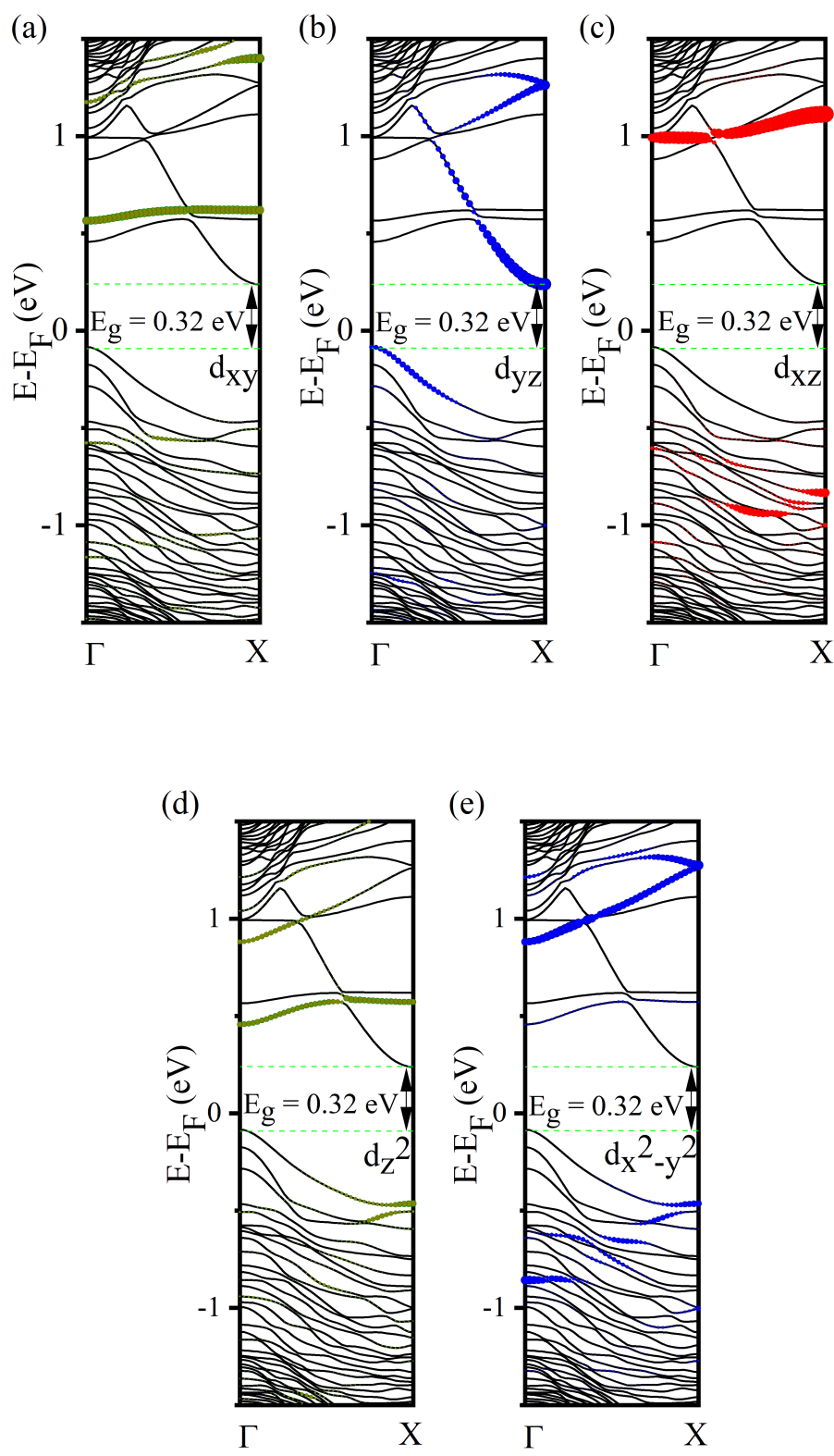

Figure 5.5: Plot showing the contribution of $\mathrm{d}_{\mathrm{xy}}, \mathrm{d}_{\mathrm{yz}}, \mathrm{d}_{\mathrm{xz}}, \mathrm{dz}^{2}$, and $\mathrm{dx}^{2}-$ $\mathrm{y}^{2}$ orbitals of $\mathrm{Cr}$ to energy bands of Cr-doped Ge-core/Si-shell nanowire (configuration I). (Reprinted with permission from Nano Lett. 2021, 21(4), 1856-1862. Copyright (2021) American Chemical Society.)

Analysis of the orbital decomposed electronic band structure (Figures 5.5 and 5.6 indicates that the interesting features found in the conduction band and bands in 

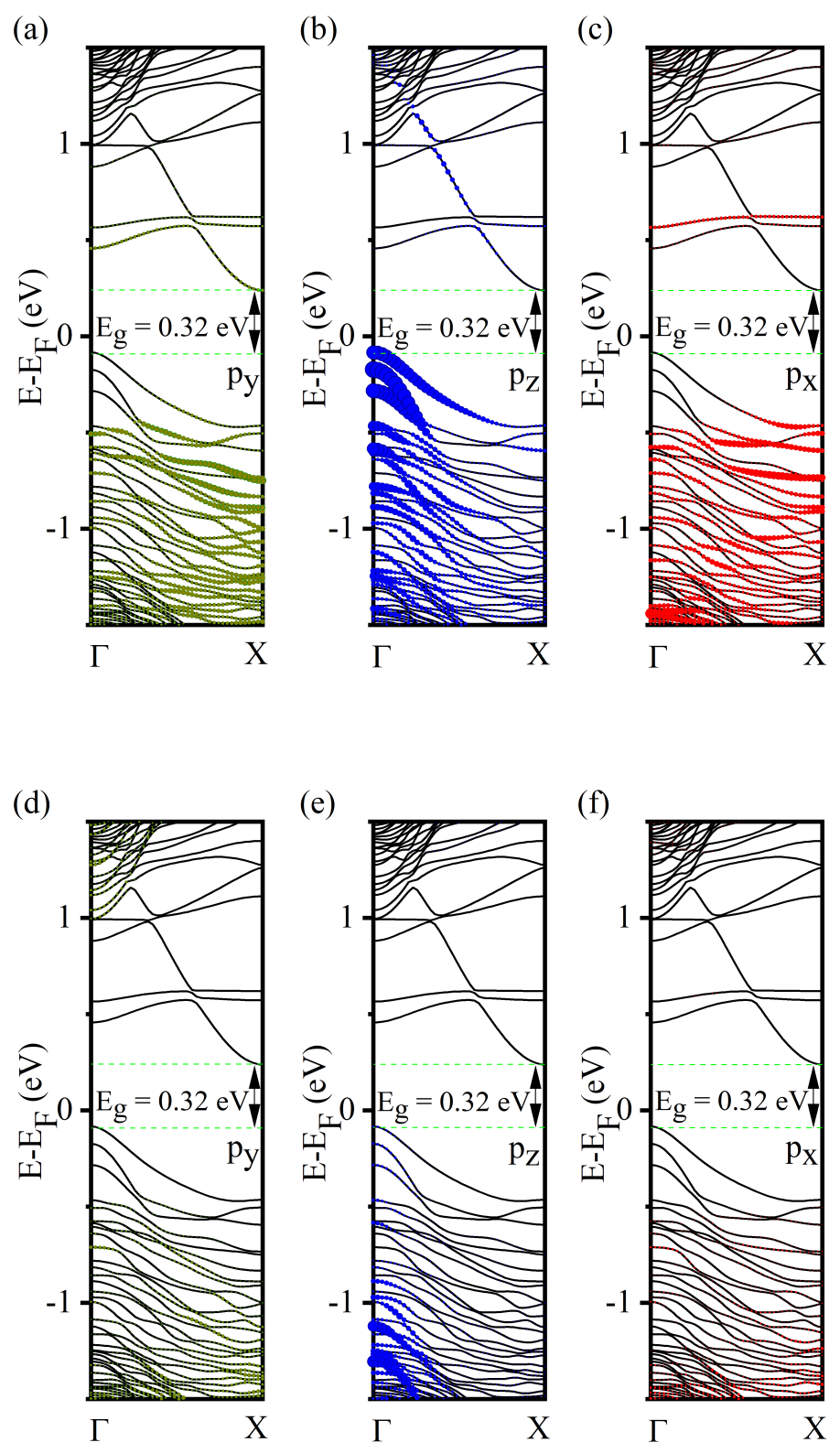

Figure 5.6: Plot showing contribution of $p_{y}, p_{z}$, and $p_{x}$ orbitals of $G e$ (a, b, and c) and Si (d, e, and f) to energy bands of Cr-doped Ge-core/Sishell nanowire (configuration I). (Reprinted with permission from Nano Lett. 2021, 21(4), 1856-1862. Copyright (2021) American Chemical Society.)

its vicinity that originated from the Cr $3 d$ states (primarily from $d_{x y}, d_{y z}$, and $d_{z^{2}}$ ) appear to be topologically relevant, specifically the non-trivial Dirac feature at the 
middle of the $\Gamma \mathrm{X}$-direction. As expected, the strain effect systematically brings these unoccupied states closer towards the Fermi level, significantly reducing the bandgap and leading to a metallic behavior at a high compressive strain of $-5.05 \%$. The shrinking in distance between the Cr-atoms under a compressive strain leads to the direct exchange superseding the super-exchange interactions causing a magnetic phase transition from an AFM to a FM state in this system.

It has been reported in earlier studies that PBE functional underestimates the bandgap in Group IV semiconductors. An accurate value for the bandgap in these materials has been obtained using the hybrid functional, HSE06[163], that contains a part of the Hartree-Fock exchange term, in addition to the exchange and correlation from the PBE functional. Therefore, to further validate the results obtained using the PBE functional, we recourse to the HSE06 functional that partially corrects the self-interaction error associated with the PBE functional. Our results show that the semiconducting behavior with the indirect bandgap feature of the nanowire is retained upon the inclusion of self-interaction error corrections. A quantitative comparison indicates an increase in the bandgap from $0.32 \mathrm{eV}$ in PBE to $0.40 \mathrm{eV}$ in HSE06 (Figure 5.7(a)). The AFM ordering between the $\mathrm{Cr}$ is preserved with the $\mathrm{d}$ orbitals of $\mathrm{Cr}$ contributing to the magnetization of $\sim \pm 3.30 \mu_{B}$; the Ge atoms bonded to the $\mathrm{Cr}$ atoms have a magnetization of $\mp 0.15 \mu_{B}$. The alignment of the spins is consistent with that obtained using the PBE functional. Next, we carried out the non-collinear magnetic calculations to explore the effects of SO interactions (Figure 5.7(b)) on the 

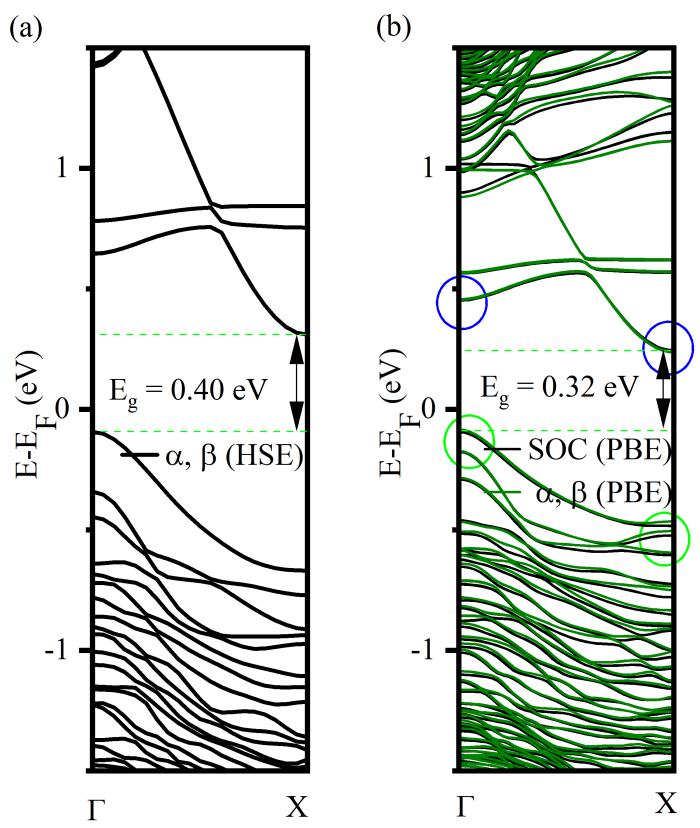

Figure 5.7: Electronic band structure of Cr-doped Ge-core/Si-shell nanowire (configuration I) obtained using (a) hybrid functional, HSE06 (b) PBE functional with and without spin-orbit coupling (SOC). The bands for spin-up and spin-down directions are degenerate. (Reprinted with permission from Nano Lett. 2021, 21(4), 1856-1862. Copyright (2021) American Chemical Society.)

electronic structure. As seen from Figure 5.7(b), the SO coupling induced shifts in the valence band (green circles) at the $\Gamma$ point and $\mathrm{X}$ point are found to be $\sim 11.16 \mathrm{meV}$ and $\sim 19.67$ meV respectively; the corresponding shifts in the conduction band (blue circles) at these points are $\sim 2.39 \mathrm{meV}$ and $\sim 7.49 \mathrm{meV}$ respectively. The small $\mathrm{SO}$ coupling effect found here is consistent with the observed weak SO coupling strength in the undoped Ge-core/Si-shell nanowire[176]. These results imply that the spin coherence length in this material can be much longer. Our non-collinear magnetic calculations further confirm that the easy axis for the spin orientation is along the 
nanowire axis with antiparallel spin alignment between the Cr-atoms; the anisotropy energy is found to vary between $0.1 \mathrm{meV}$ and $0.4 \mathrm{meV}$, which is consistent with the observed anisotropy energy in AFM materials with ultrafast spin dynamics [177].

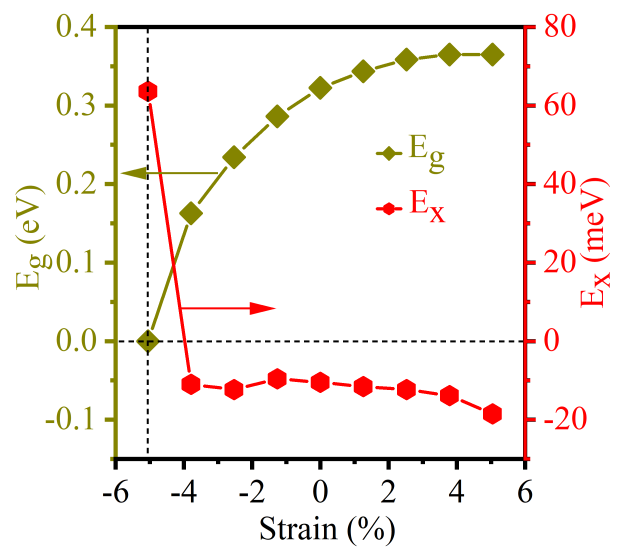

Figure 5.8: Variation of bandgap and exchange energy with strain in the Cr-doped Ge-core/Si-shell nanowire (configuration I). The strain in percentage is calculated as: $\operatorname{strain}(\%)=\left(\frac{a-a_{0}}{a_{0}}\right) \times 100 \%$, where $a_{0}$ is the equilibrium lattice parameter. (Reprinted with permission from Nano Lett. 2021, 21(4), 1856-1862. Copyright (2021) American Chemical Society.)

The implantation of transition metal dopants into $\mathrm{Si}$ and Ge nanowires is a nonequilibrium process (due to their low solubility) usually done at high temperature $\left(350^{\circ} \mathrm{C}-800^{\circ} \mathrm{C}\right)[50]$, which can induce external strains in these systems during synthesis. Thus, we focused next on the effects of tensile and compressive strains on the electronic structure and magnetic properties of these nanowires. The variation of the energy gap and exchange energy with lattice strain is presented in Figure 5.8. We notice an increase in bandgap from $0.32 \mathrm{eV}$ at $0 \%$ strain to $0.37 \mathrm{eV}$ at $+5.0 \%$ strain (Figures 5.9(a), 5.9(b), 5.9(c), and 5.9(d)). 

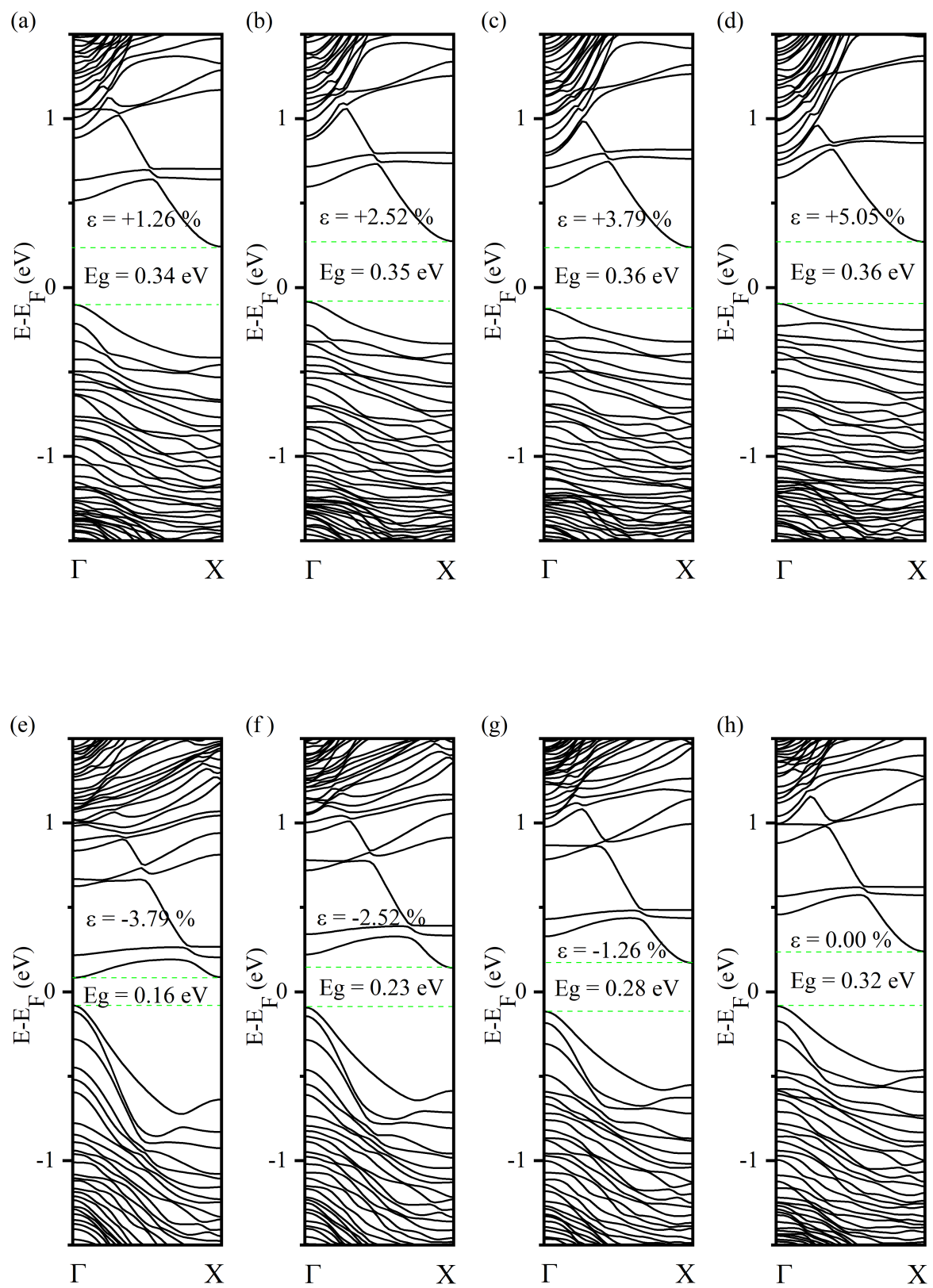

Figure 5.9: Electronic band structure (PBE) of Cr-doped Ge-core/Sishell nanowire (configuration I) at different values of $(\mathrm{a}-\mathrm{d})$ tensile $(+1.26 \%$, $+2.52 \%,+3.79 \%$, and $+5.05 \%$ ) (e-g) compressive $(-3.79 \%,-2.52 \%,-1.26 \%$, and $0.00 \%$ ) strains. (h) represents the electronic band structure at $0.00 \%$ strain. The bands for spin-up and spin-down directions are degenerate. (Reprinted with permission from Nano Lett. 2021, 21(4), 1856-1862. Copyright (2021) American Chemical Society.) 

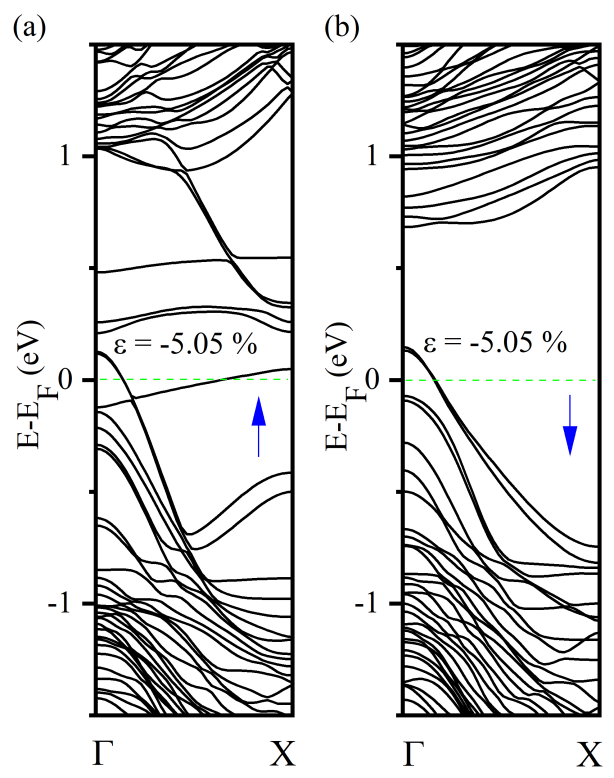

Figure 5.10: Electronic band structure (PBE) of Cr-doped Ge-core/Si-shell nanowire (configuration I) for the (a) spin-up (b) spin-down direction at a compressive strain of $-5.05 \%$. (Reprinted with permission from Nano Lett. 2021, 21(4), 1856-1862. Copyright (2021) American Chemical Society.)

The energy gaps remain indirect, and the nanowire prefers to be in an AFM phase. For a compressive strain (Figures 5.9 (e), 5.9(f), and 5.9 (g)), the indirect nature of the energy gap is preserved up to a strain of $-2.52 \%$, beyond which, an indirect to direct bandgap transition is observed. At a strain of $-5.05 \%$, a metallic behavior was noted (Figure 5.10). Unlike the semiconducting phase, which is AFM, the metallic phase is FM with an exchange energy of $64 \mathrm{meV}$.

Motivated by the observed weak spin-orbit interactions and negligible strain effects (for strain $< \pm 1.26 \%$ ) on the energy levels at the vicinity of the Fermi energy, we model a two-terminal junction to access its possible usage in spintronics. The 
portion of the Cr-doped Ge-core/Si-shell nanowire was sandwiched between two gold electrodes to construct the prototype; the electrode-electrode distance was $\sim 17 \AA$.
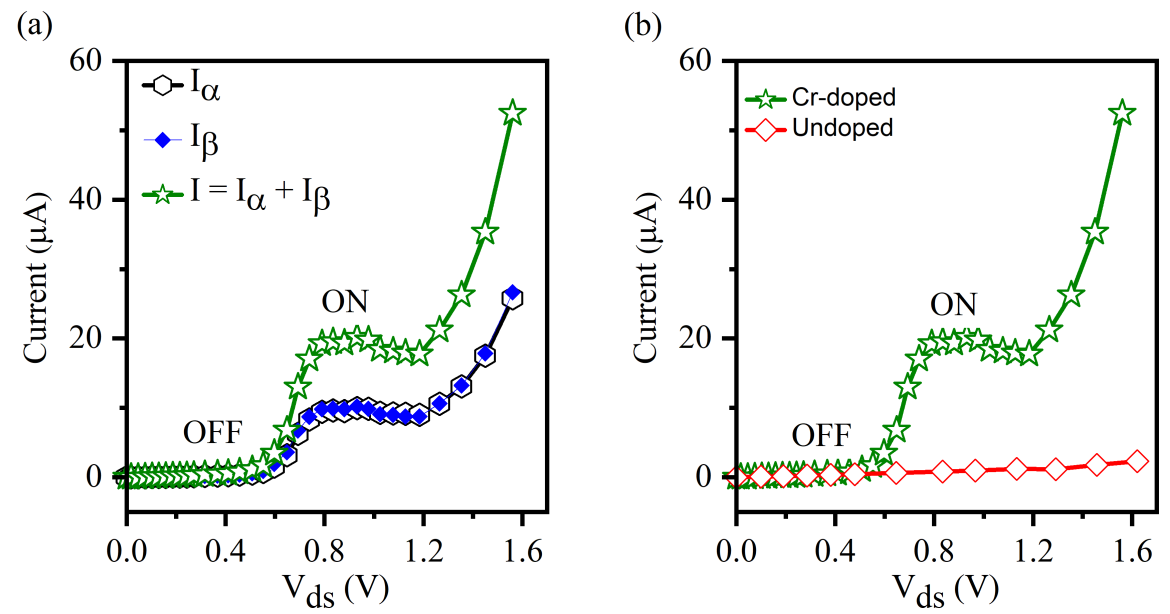

Figure 5.11: (a) Current (I)-Voltage $\left(\mathrm{V}_{\mathrm{ds}}\right)$ characteristics in a two-terminal Cr-doped Ge-core/Si-shell nanowire junction; spin-up $\left(\mathrm{I}_{\alpha}\right)$ and spin-down $\left(\mathrm{I}_{\beta}\right)$ currents are identical. (b) Comparison of $\mathrm{I}-\mathrm{V}$ characteristics between undoped and Cr-doped nanowire junction. (Reprinted with permission from Nano Lett. 2021, 21(4), 1856-1862. Copyright (2021) American Chemical Society.)

Figure 5.11 shows the calculated spin-polarized current in the junction; the current has been calculated using Equation 5.2. The I-V curve (Figure 5.11(a)) has three features: (i) the current is negligible for voltage ranging from $0 \mathrm{~V}$ to $0.6 \mathrm{~V}$, which we refer to as the OFF state, (ii) there is almost a plateau behavior in current (ON state) for the voltage range $(0.74 \mathrm{~V}-1.20 \mathrm{~V})$, and (iii) current rises sharply for bias beyond $1.2 \mathrm{~V}$. The observed $\mathrm{ON}$ and $\mathrm{OFF}$ state feature indicates that the Cr-doped Gecore/Si-shell nanowire can be used as a two-terminal switch. The ON/OFF current ratio is found to be $\sim 41$ at a bias of $0.83 \mathrm{~V}$. The current for the Cr-doped nanowire 

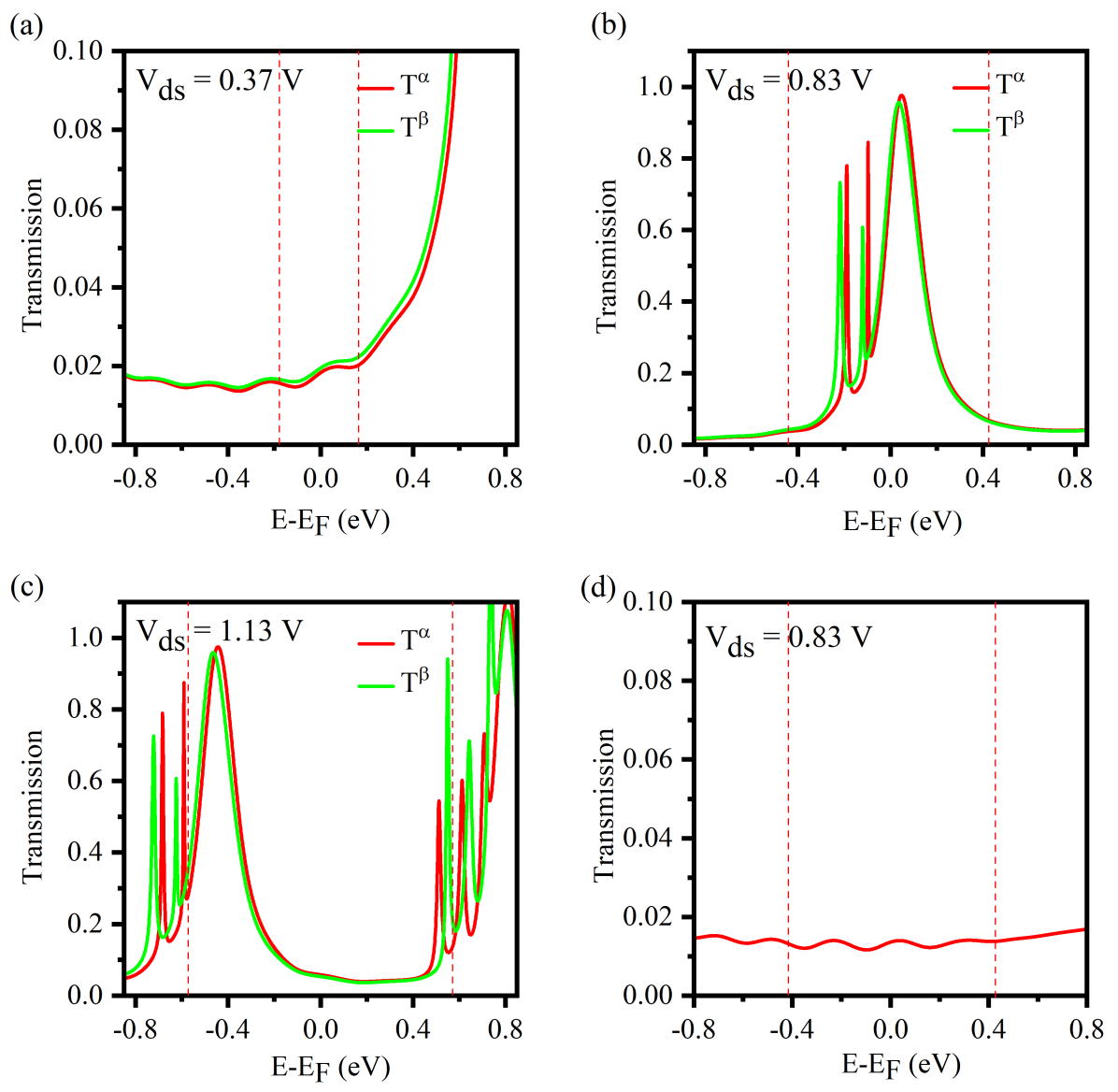

Figure 5.12: (a-c) Bias-dependent spin-polarized transmission, $\mathrm{T}^{\alpha, \beta}(\mathrm{E}, \mathrm{V})$, as a function of injection energy in the Cr-doped core-shell nanowire junction. (d) Transmission as a function of injection energy for undoped nanowire junction. In all figures, the chemical potential windows (for integration) are represented by the dotted lines (red).

(Figure $5.11(\mathrm{~b}))$ is found to be significantly higher $(\sim 24$ times at $0.83 \mathrm{~V})$ than that observed in the undoped nanowire, suggesting that the Cr-dopant is responsible for the observed switching behavior. To unravel the origin of switching, we calculated the spin-polarized $T^{\sigma}(E, V)$ as a function of injection energy at different bias points (Figure 5.12). The smaller observed current (OFF state) at the $0.37 \mathrm{~V}$ as compared 
to the $0.83 \mathrm{~V}$ can be understood from the much smaller transmission value $(\sim 0.02)$ in Figure 5.12(a) within the integration window. In contrast, three transmission peaks (peak heights $>0.6)$ that appear within the chemical potential window $[-0.42 \mathrm{eV}$, $0.42 \mathrm{eV}]$ in Figure 5.12 (b) for a bias of $0.83 \mathrm{~V}$ explains the ON state behavior in the current. To understand a small drop in current at $1.13 \mathrm{~V}$ as compared to 0.83 $\mathrm{V}$, we analyzed the transmission function at $1.13 \mathrm{~V}$ (Figure $5.12(\mathrm{c})$ ). A comparison of transmission values indicates the three transmission peaks that appeared at 0.83 $\mathrm{V}$ within the chemical potential window $[-0.42 \mathrm{eV}, 0.42 \mathrm{eV}]$ shifted to the left in Figure 5.12(c) due to Stark effect, resulting in a smaller area under the transmission curves and hence a smaller current. Furthermore, to understand the higher observed current in the Cr-doped nanowire junction $(\sim 24$ times at $0.83 \mathrm{~V})$ as compared to undoped ones, we plotted the transmission function for the undoped Ge-core/Si-shell nanowire device at a bias voltage of $0.83 \mathrm{~V}$ (Figure $5.12(\mathrm{~d})$ ). A much smaller value of transmission $(\sim 0.02)$ within the chemical potential window $[-0.40 \mathrm{eV}, 0.43 \mathrm{eV}]$ in the undoped nanowire as compared to a higher transmission value $\sim 1$ for the Cr-doped junction explains the much smaller current found in the undoped nanowire. Thus, the question again arises: What does contribute to the higher transmission in the Crdoped nanowire junction? To answer this, we have calculated the projected density of states (DOS) per atom in the device configuration at $0.83 \mathrm{~V}$. For the undoped device (Figure 5.13(a)), the contribution to the transmission comes mostly from the Ge atoms, but it is still insignificant $(\sim 0.09$ at the Fermi energy). For the Cr-doped 

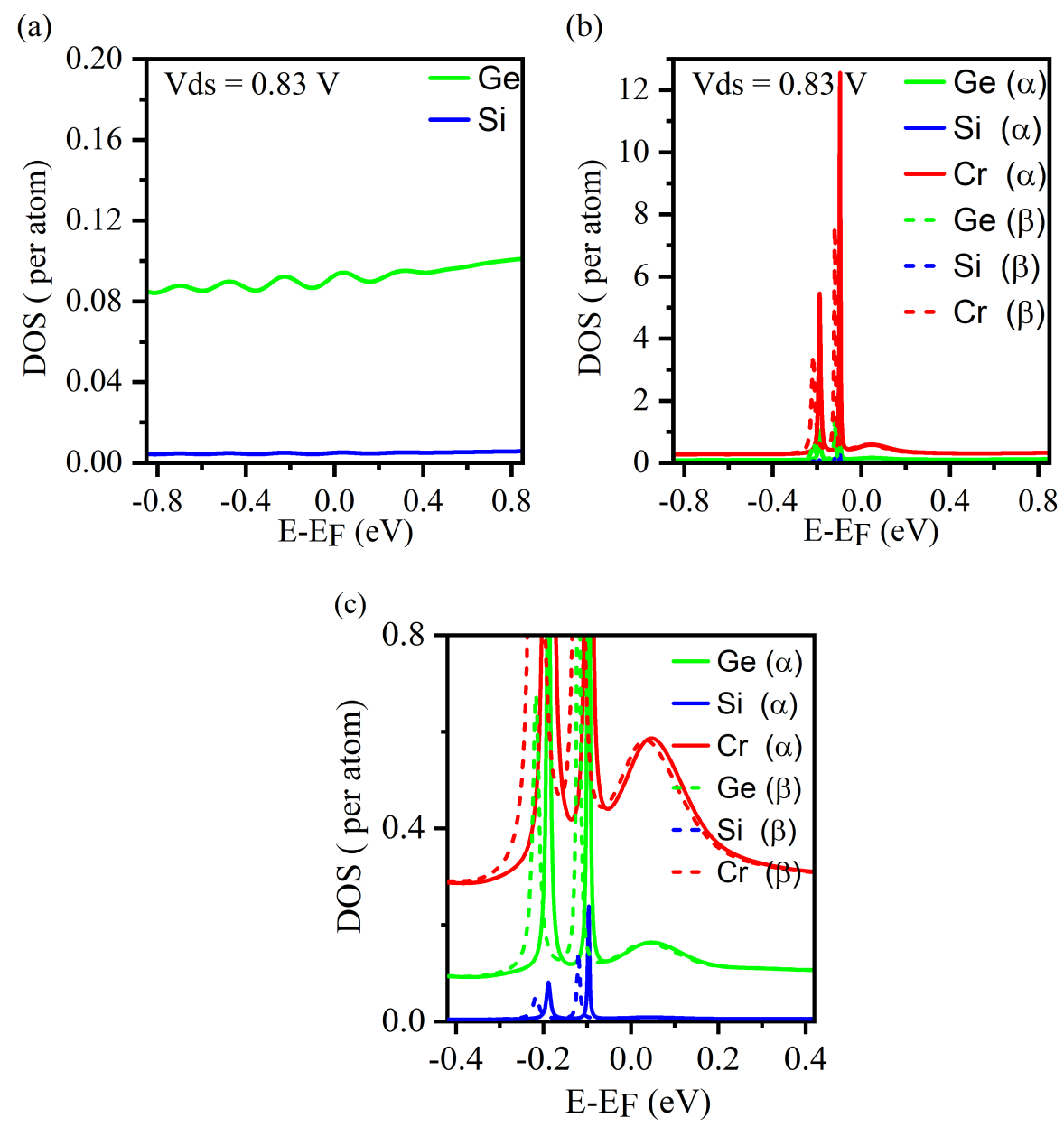

Figure 5.13: Projected atom decomposed spin-polarized density of states (DOS) for (a) undoped (b) Cr-doped nanowire junction; (c) is the magnified version of (b). (Reprinted with permission from Nano Lett. 2021, 21(4), 1856-1862. Copyright (2021) American Chemical Society.)

junction (Figures 5.13(b) and 5.13(c)), on the other hand, a significant contribution to the density of states (DOS) comes from the Cr atoms at the Fermi energy. This clearly confirms the role of Cr-dopants in dictating the switching behavior in doped nanowire circuits. 


\subsection{Conclusions}

We report a comprehensive study that reveals the Cr-doped Ge-core/Si-shell nanowire as an AFM semiconductor. The superexchange interactions mediated by the $\mathrm{p}_{\mathrm{z}}$ orbitals of the nearby Ge is found to be responsible for the AFM ordering of spins between $\mathrm{Cr}$ (localized $\mathrm{d}_{\mathrm{yz}}$ orbitals). The noncollinear magnetic calculations confirm the weak SO interactions in the nanowire, suggesting that the spins can be transported coherently over a long distance in this material. The valence band offset between the Ge-core and Si-shell observed in undoped Ge-core/Si-shell nanowire remains intact upon the substitutional doping of Cr. The spin-dependent quantum transport calculations in a model nanowire junction show a switching behavior with a high ON/OFF current ratio. Considering the significant progress being made in recent years in coreshell nanowire technology and ion implantation technique, we expect our prediction to initiate experimental interest in the Cr-doped Ge-core/Si-shell nanowire for potential applications in low-dimensional AFM semiconductor spintronics. 


\section{Chapter 6}

\section{$\mathrm{PbTe} / \mathrm{PbS}$ core/shell nanowire}

\subsection{Introduction}

Lead salts $\mathrm{PbX}(\mathrm{X}=\mathrm{Te}, \mathrm{Se}$, and $\mathrm{S})$ have drawn substantial interests in recent years due to their possible applications in infrared lasers [178, 179, 180], sensors [181], longdistance fiber-optic communications [179], thermophotovoltaics[182], and thermoelectric devices [183, 184. The 3D bulk phase of these materials have a small bandgap $\leq 0.3 \mathrm{eV}$. However, their energy gap can be tuned significantly by reducing the dimension of the bulk material that escalates the quantum effects; the magnitude of the bandgap depends upon the size and shape of these nanostructures as reported 
in a number of previous studies[185, 186]. The ability of synthesizing high quality nanostructures [71, 72, 73, 74, 75] of these materials with varying shape and size make them suitable candidate for potential applications including but not limited to field-effect transistors (FETs) [76, 777, photodetectors[187], solar cells[78], highperformance optoelectronics [79, 80], as well as in high-efficiency thermoelectrics [72].

On the other hand, the core/shell nanostructures [42, 188, 189, 190, 191] have immense benefits. For instance, it has been found that the stability of the nanostructures against oxidation can be enhanced by shelling them with materials that have larger bandgap[67]. This (shelling) increases the performance of solar cells and lightemitting diodes (LEDs) by substantially reducing the trap states, diminishing the nonradiative recombination [68] processes, and enhancing the carrier multiplication [69] and carrier transport processes[70]. In an another study, the core/shell geometry has been found to enhance the luminescence in materials[192]. Moreover, the core/shell nanowire field effect transistors (FETs) show superior performance compared to the state of art metal oxide semiconductor field effect transistor (MOSFETs) [37]; the programmable logic circuit of core/shell nanowire FETs have also been demonstrated in previous studies[43]. In a recent theoretical study, it has been proposed that the Mn-doped core/shell nanowire can act as an excellent spin filter with spin filtering efficiency of more than 90\%[57] and Cr-doped core/shell heterostructures can act as a switch[193]. Furthermore, these materials are beneficial for catalytic applications from intensifying the $H_{2}$ generation process 194 to acting as an electrocatalyst for 
oxidation reduction[195, 196].

In this work, we have explored the electronic structure, thermodynamic stability, mechanical, and optical properties of $\mathrm{PbTe} / \mathrm{PbS}$ core/shell nanowires of different core diameters along $\langle 200\rangle$ and $\langle 111\rangle$ directions using the first-principles plane-wave density functional theory (DFT). These heterostructures are found to be indirect (direct) bandgap semiconductors in the $\langle 200\rangle(\langle 111\rangle)$ direction; the bandgap can be easily tuned by changing the diameter of the core. The inclusion of the spinorbit coupling (SOC) to our calculations is found to lower the bandgap of these materials. The minimum of the conduction band $(\mathrm{CB})$, which is mostly $\mathrm{Pb}$, moves towards the Fermi level in the presence of SOC without changing much the valence band (VB) maximum. Nanowires along the $\langle 200\rangle$ direction have higher value of the Youngs's modulus $(\sim 48 \mathrm{GPa})$ compared to the wires in the $\langle 111\rangle(\sim 20 \mathrm{GPa})$ direction; these values do not change significantly with the thickness of the core. Higher compressive strains $(>11.30 \%)$ can cause a semiconductor to metallic phase transitions in these nanowires with the pressure for such phase transitions being in the range of $\sim 3 \mathrm{GPa}$ to $\sim 6 \mathrm{GPa}$, consistent with the experimental observation in $\mathrm{PbS}$ nanowires and nanoparticles. The absorption spectrum for these materials is broad $(0.39 \mathrm{eV}-13 \mathrm{eV})$ that includes the infrared-visible-ultraviolet region. The ab-initio molecular dynamics calculations show that core/shell structures in the $\langle 200\rangle$ direction is less prone to core to shell diffusion compared to the $\langle 111\rangle$ direction irrespective of the thickness of the core and shell. 


\subsection{Computational Details}

The lead salts $\mathrm{PbX}(\mathrm{X}=\mathrm{Te}, \mathrm{S})$ crystallize in the $\mathrm{NaCl}$ type structure (lattice type: FCC cubic) with space group $F m \overline{3} m$. From the bulk structure of $\mathrm{PbTe}$ and $\mathrm{PbS}$, we constructed pristine $\mathrm{PbTe}$ and $\mathrm{PbS}$ nanowires in the $\langle 200\rangle$ and $\langle 111\rangle$ directions. To construct the $\mathrm{PbTe} / \mathrm{PbS}$ core/shell nanowire heterostructures, we replaced the $\mathrm{Te}$ atoms in the shell with $\mathrm{S}$ atoms. We then enclosed each of these heterostructures in a huge supercell that is periodic in the longitudinal direction (nanowire axis) but has a large lattice constant of $35 \AA$ in the transverse directions. This was done to diminish the effect of the surplus interactions between the nanowires and their periodic replica. Each of these supercells considered here has 74 atoms. In the pristine $\mathrm{PbTe}$ (PbS) nanowires, there are $37 \mathrm{~Pb}$ and $37 \mathrm{Te}(\mathrm{S})$ atoms. In the $\mathrm{PbTe} / \mathrm{PbS}$ core/shell heterostructures, there are $37 \mathrm{~Pb}$ atoms, with the remaining 37 being the sum of $\mathrm{Te}$ and S atoms. The small (big) core nanowires in the $\langle 200\rangle$ direction has 5 (13) Te and 32 (24) S atoms. Similarly, the small (big) core nanowires have 7 (19) Te and 30 (18) S atoms along the $\langle 111\rangle$ direction. Consequently, we optimized each of these nanowire structures without symmetry constraints until the residual force on each atom is less than $0.01 \mathrm{eV} / \AA$ using the plane-wave density functional theory (DFT) implemented in VASP [157, 158]. Figures 6.1 and 6.2 illustrate the optimized unit cell structures of the pristine $\mathrm{PbTe}$ and $\mathrm{PbS}$ nanowire structures. The interactions between ionic core and valence electrons in our calculations were treated using the 
(a)

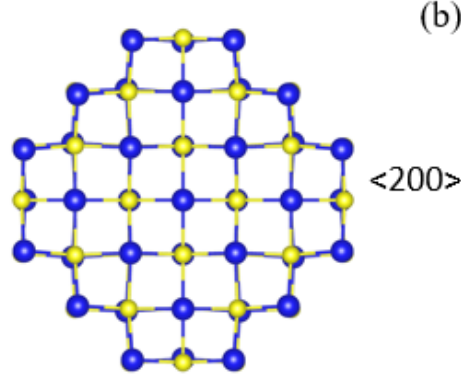

(c)

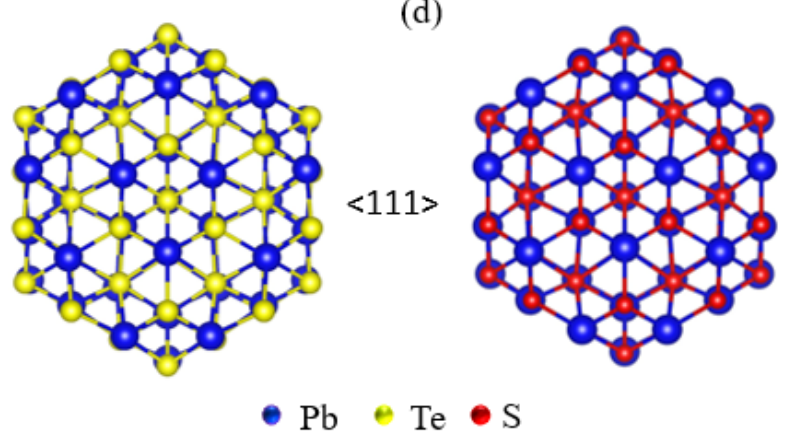

Figure 6.1: The unit cell structures of pristine (a) PbTe (b) PbS nanowire along the $\langle 200\rangle$ direction. Similarly, (c) and (d) illustrate the pristine $\mathrm{PbTe}$ and $\mathrm{PbS}$ nanowire unit cell structures in the $\langle 111\rangle$ direction. (Reprinted with permission from J. Phys. Chem. C - https://doi.org/10.1021/acs.jpcc.1c06577. Copyright (2021) American Chemical Society.)

projector augmented wave (PAW) [118, 122] method. We have approximated the exchange-correlation energy using the Perdew-Burke-Ernzerhof (PBE) functional that lies within the framework of the generalized gradient approximation (GGA)[105]. Monkhrost-Pack (MP) [113, 114] k-point grid of $1 \times 1 \times 7(7 \times 1 \times 1)$ was used to sample the Brillouin zone for the nanowires oriented along the $\langle 111\rangle(\langle 200\rangle)$ direction. Based on the convergence tests for the cutoff energy, we used an energy cut-off value of 400 $\mathrm{eV}$. The convergence critera for the total energy was set at $10^{-6} \mathrm{eV}$. The PbTe $(\mathrm{PbS})$ nanowire in the $\langle 200\rangle$ has equilibrium lattice parameter of $6.58 \AA(6.06 \AA)$. In the 
(a)

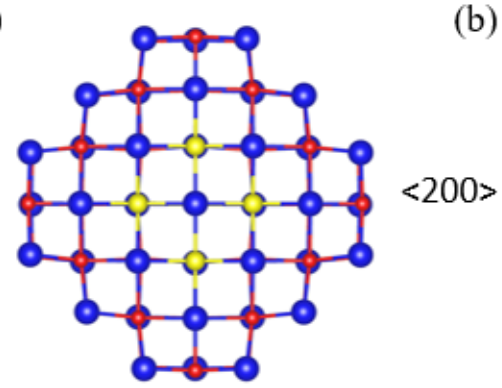

(c)

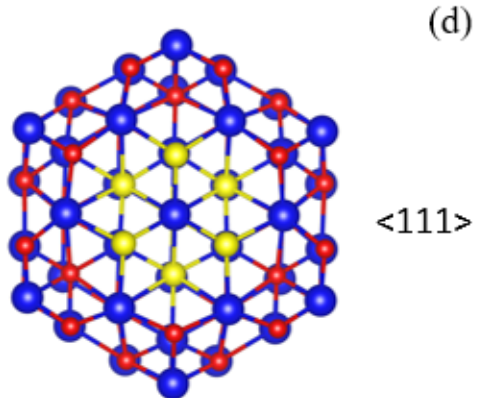

(b)

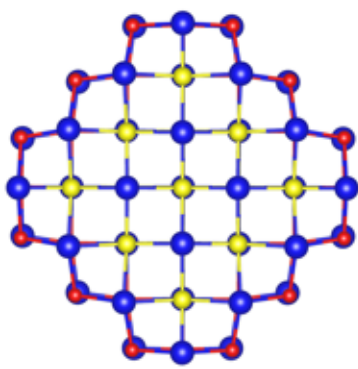

(d)

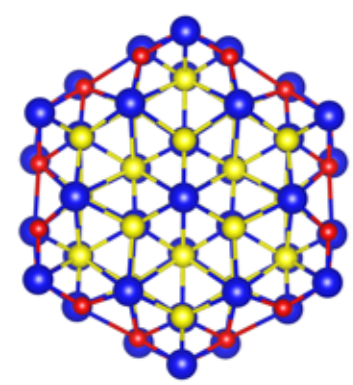

- $\mathrm{Pb} \oplus \mathrm{Te} \bullet \mathrm{S}$

Figure 6.2: The unit cell structures of (a) small core $\left(d_{c} \sim 6.32 \AA\right)$ (b) big core $\left(d_{c} \sim 12.82 \AA\right) \mathrm{PbTe} / \mathrm{PbS}$ core/shell nanowire along the $\langle 200\rangle$ direction. Similarly, $(\mathrm{c})$ and $(\mathrm{d})$ illustrate the small core $\left(d_{c} \sim 5.13 \AA\right)$ and big core $\left(d_{c} \sim 10.83 \AA\right) \mathrm{PbTe} / \mathrm{PbS}$ core/shell unit cell structures in the $\langle 111\rangle$ direction. (Reprinted with permission from J. Phys. Chem. C - https://doi.org/10.1021/acs.jpcc.1c06577. Copyright (2021) American Chemical Society.)

$\langle 111\rangle$ direction, the equilibrium lattice constant is $11.31 \AA(10.35 \AA)$ for the $\mathrm{PbTe}$ $(\mathrm{PbS})$ nanowire. Similarly, the small (big) core $\mathrm{PbTe} / \mathrm{PbS}$ nanowire in the $\langle 200\rangle$ direction has equilibrium lattice constant of $6.25 \AA(6.37 \AA)$. We obtain the lattice constant of $10.53 \AA(11.11 \AA)$ for the small (big) PbTe/PbS nanowire in the $\langle 111\rangle$ direction.

In this work, the electronic structure, mechanical and optical properties of these 
nanowires have been studied both with and without spin-orbit interactions. In addition, we performed the Born-Oppenheimer ab initio molecular dynamics (MD) [197] calculations using the Nose-Hoover thermostat[197] in NVT ensmeble as implemented in VASP to study the thermodynamic stability of these core/shell structures at room temperature $(300 \mathrm{~K})$; the total number of time steps for the MD calculations was 4000 with a time resolution of $2 \mathrm{fs}$.

\subsection{Results and discussion}

(a)

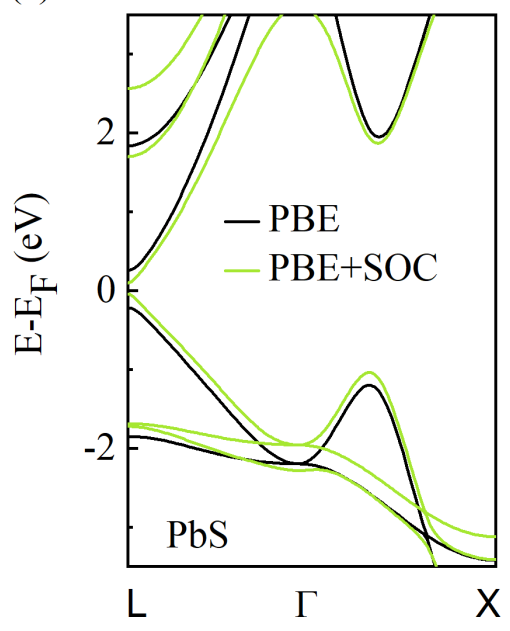

(b)

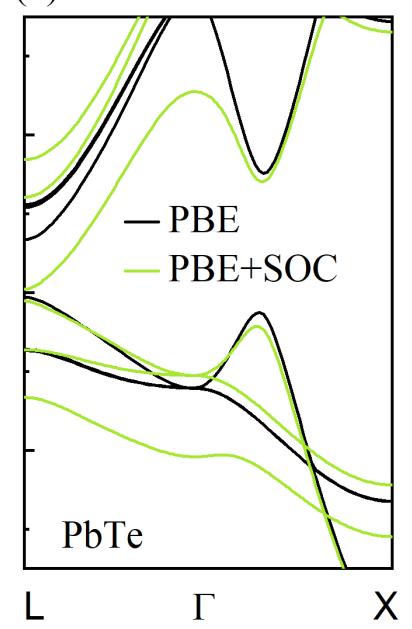

Figure 6.3: The electronic band structure of bulk (a) PbS (b) PbTe obtained using PBE and PBE+SOC. (Reprinted with permission from J. Phys. Chem. C - https://doi.org/10.1021/acs.jpcc.1c06577. Copyright (2021) American Chemical Society.)

We start with analyzing the results of our calculations for lead salts $\mathrm{PbX}(\mathrm{X}=\mathrm{Te}$ and S) obtained using the PBE and PBE+SOC. The relaxed unit cell structures have 
lattice constant $\sim 6.56 \AA$ and $\sim 5.99 \AA$ for bulk PbTe and PbS, respectively. The electronic band structure of bulk $\mathrm{PbX}(\mathrm{X}=\mathrm{Te}$ and $\mathrm{S})$, shown in Figure 6.3, reveals that these materials are direct bandgap (L-point) semiconductor. We obtain bandgap $\sim 0.83 \mathrm{eV}(\sim 0.15 \mathrm{eV})$ at the L-point using $\mathrm{PBE}(\mathrm{PBE}+\mathrm{SOC})$ for the bulk PbTe; the energy gap obtained using $\mathrm{PBE}+\mathrm{SOC}$ agrees well with the reported gap of $\sim 0.19$ eV[198, 199, 200] for this material. Similarly, PBE (PBE+SOC) functional gives an energy gap $\sim 0.47 \mathrm{eV}(\sim 0.14 \mathrm{eV})$ at the L-point for bulk PbS, indicating that the reported gap of $\sim 0.29 \mathrm{eV}[199,200,201$ lies within the gaps obtained using PBE and $\mathrm{PBE}+\mathrm{SOC}$ for bulk PbS.
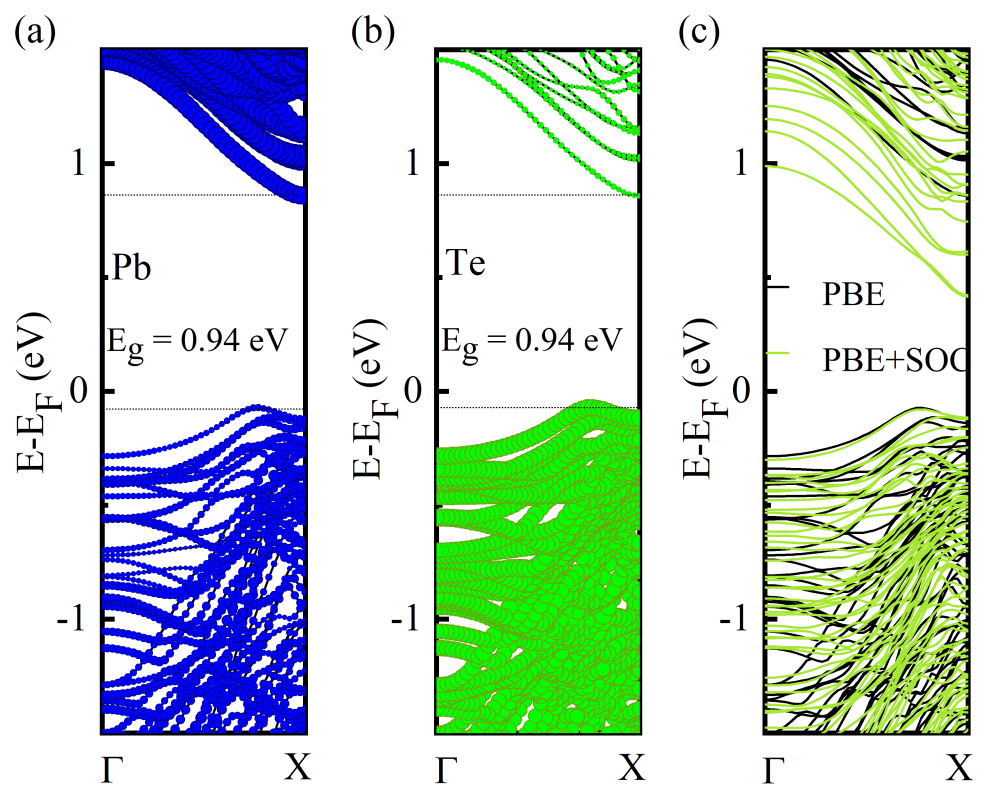

Figure 6.4: ( $\mathrm{a}$ and $\mathrm{b}$ ) The atom decomposed electronic band structure $(\mathrm{PBE})$ of $\mathrm{PbTe}$ nanowire in the $\langle 200\rangle$ direction; the circle represents the contribution of the $\mathrm{Pb}$ and Te atoms to energy bands. The electronic band structures of PbTe nanowire obtained using $\mathrm{PBE}$ and $\mathrm{PBE}+\mathrm{SOC}$ are compared in (c). (Reprinted with permission from J. Phys. Chem. C - https://doi.org/10.1021/acs.jpcc.1c06577. Copyright (2021) American Chemical Society.) 
Next, we discuss the electronic band structure of pristine PbTe nanowire along the $\langle 200\rangle$ and $\langle 111\rangle$ directions. Figures 6.4 and 6.5 reveal that $\mathrm{PbTe}$ nanowire is an indirect bandgap semiconductor irrespective of the directions considered. The PBE functional gives an energy gap of $\sim 0.94 \mathrm{eV}(\sim 0.84)$ for PbTe nanowire in the $\langle 200\rangle$ $(\langle 111\rangle)$ direction. In presence of SOC, the gap along the $\langle 200\rangle(\langle 111\rangle)$ direction is reduced to $\sim 0.49 \mathrm{eV}(\sim 0.48 \mathrm{eV})$. Similarly, $\mathrm{PbS}$ nanowire is a direct bandgap semi-
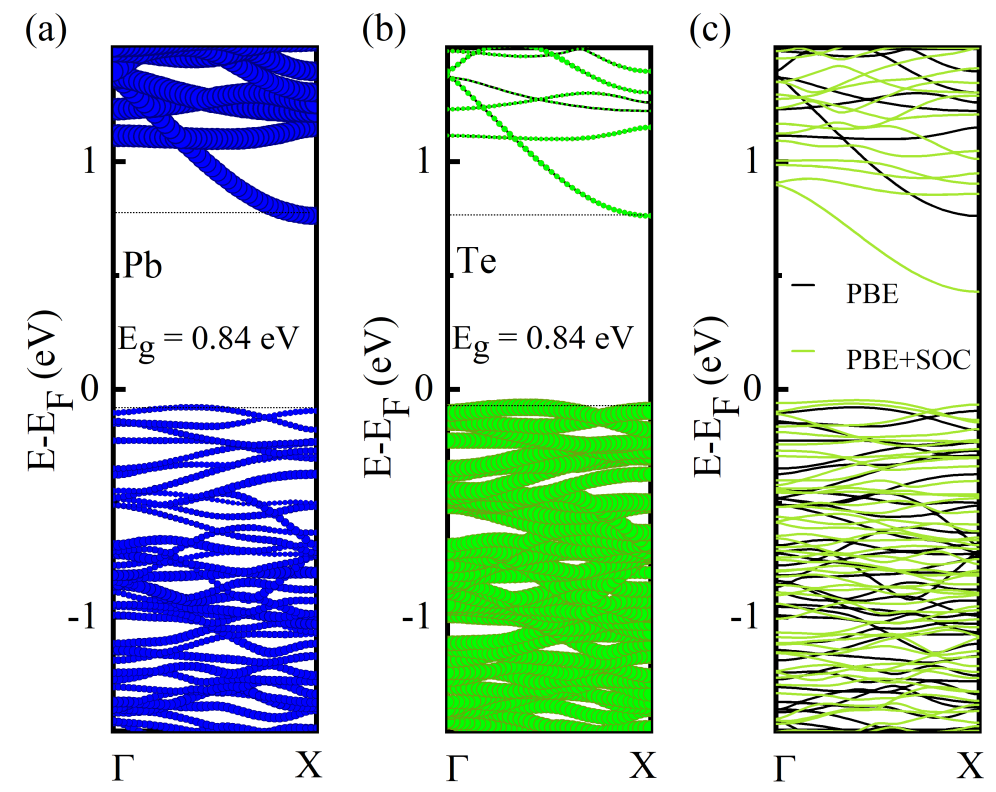

Figure 6.5: ( $\mathrm{a}$ and $\mathrm{b}$ ) The atom decomposed electronic band structure (PBE) of $\mathrm{PbTe}$ nanowire in the $\langle 111\rangle$ direction; the circle represents the contribution of the $\mathrm{Pb}$ and $\mathrm{Te}$ atoms to energy bands. The electronic band structures of $\mathrm{PbTe}$ nanowire obtained using $\mathrm{PBE}$ and $\mathrm{PBE}+\mathrm{SOC}$ are compared in (c). (Reprinted with permission from J. Phys. Chem. C - https://doi.org/10.1021/acs.jpcc.1c06577. Copyright (2021) American Chemical Society.)

conductor (Figures 6.6 and 6.7) with an energy gap of $\sim 1.05 \mathrm{eV}(\sim 0.82 \mathrm{eV})$ in the $\langle 200\rangle(\langle 111\rangle)$ direction without SOC. The bandgap in the presence of SOC becomes 
$\sim 0.78 \mathrm{eV}(\sim 0.58 \mathrm{eV})$ in the $\langle 200\rangle(\langle 111\rangle)$ direction. Such reduction of bandgap in the presence of $\mathrm{SOC}$ is due to the lowering of the $\mathrm{CB}$ of these systems. It may be noted that the VB does not change much in the presence of SOC. This is evident as the $\mathrm{CB}$ is dominated by $\mathrm{Pb}$ atoms and the valence band by $\mathrm{Te}(\mathrm{S})$ atoms. From our analysis of electronic band structure for bulk phase of $\mathrm{PbTe}$, we expect $\mathrm{PBE}+\mathrm{SOC}$ to give a reasonable value of the energy gap for PbTe nanowires. Similarly, the bandgap of the $\mathrm{PbS}$ is expected to lie within the $\mathrm{PBE}$ and $\mathrm{PBE}+\mathrm{SOC}$ gaps.
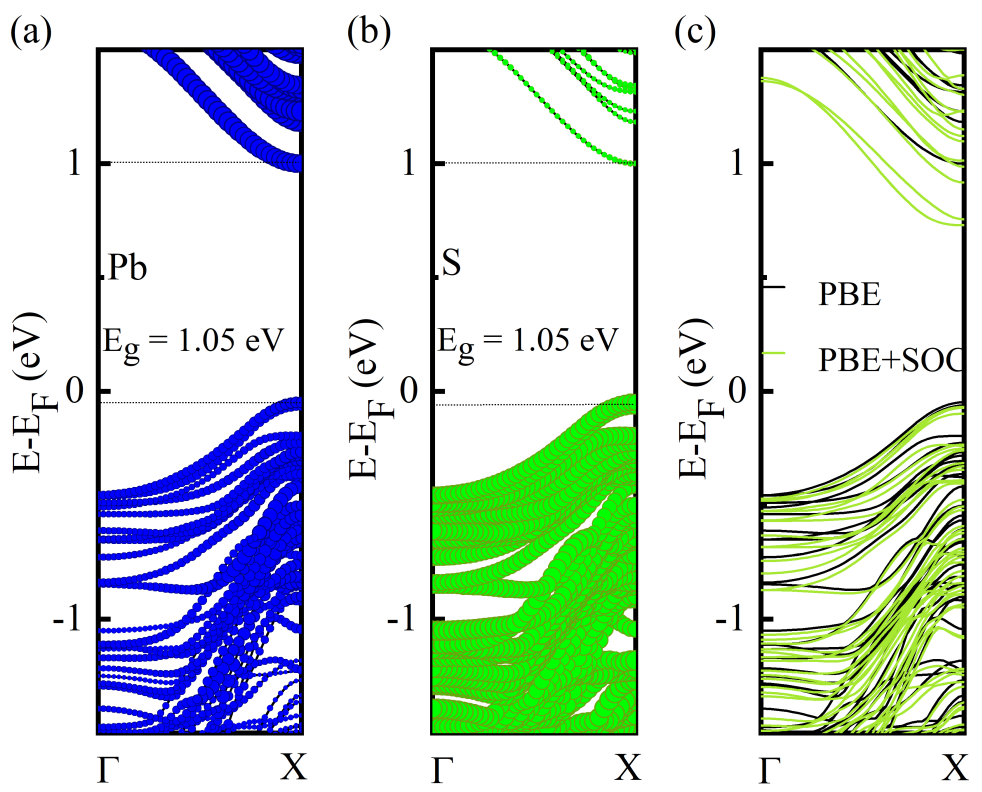

Figure 6.6: (a and b) The atom decomposed electronic band structure $(\mathrm{PBE})$ of $\mathrm{PbS}$ nanowire in the $\langle 200\rangle$ direction; the circle represents the contribution of the $\mathrm{Pb}$ and $\mathrm{S}$ atoms to energy bands. The electronic band structures of $\mathrm{PbS}$ nanowire obtained using $\mathrm{PBE}$ and $\mathrm{PBE}+\mathrm{SOC}$ are compared in (c). (Reprinted with permission from J. Phys. Chem. C - https://doi.org/10.1021/acs.jpcc.1c06577. Copyright (2021) American Chemical Society.)

Recently, there has been a lot of experimental studies on the lead chalcogenides PbX 

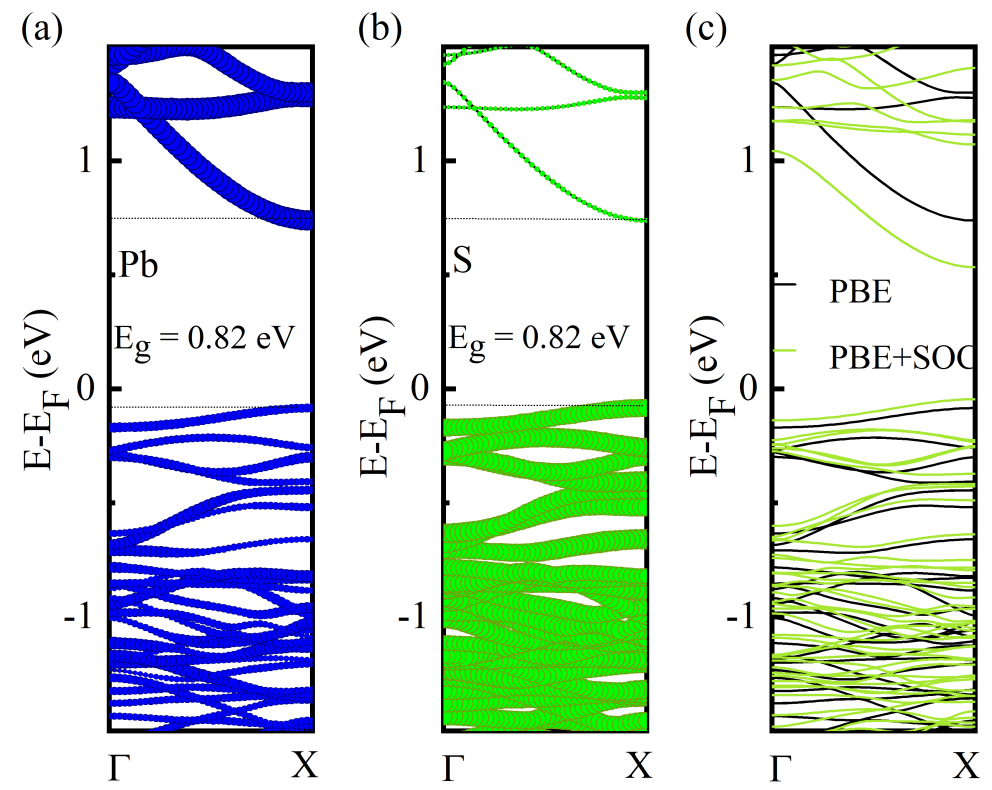

Figure 6.7: (a and b) The atom decomposed electronic band structure (PBE) of $\mathrm{PbS}$ nanowire in the $\langle 111\rangle$ direction; the circle represents the contribution of the $\mathrm{Pb}$ and $\mathrm{S}$ atoms to energy bands. The electronic band structures of $\mathrm{PbS}$ nanowire obtained using $\mathrm{PBE}$ and $\mathrm{PBE}+\mathrm{SOC}$ are compared in (c). (Reprinted with permission from J. Phys. Chem. C - https://doi.org/10.1021/acs.jpcc.1c06577. Copyright (2021) American Chemical Society.)

$(\mathrm{X}=\mathrm{Te}, \mathrm{Se}$, and $\mathrm{S})$ nanostructures. As reported in such studies, shelling the lead chalcogenides nanostructures with a material of higher bandgap (also lead chalcogenides) increases their stability against oxidation, reduces the surface trap states, diminish the nonradiative recombination process, and enhances the carrier multiplication and carrier transport processes. This has led to the superior performance of the optoelectronic and thermoelectric devices. Therefore, in this work, we study the $\mathrm{PbTe} / \mathrm{PbS}$ core/shell nanowires of different core diameters along the $\langle 200\rangle$ and $\langle 111\rangle$ directions as less effort has been made to understand their electronic band structure, 
stability, mechanical, and optical properties. In the $\langle 200\rangle$ direction, the small (big) core nanowire has core diameter of $\sim 6.32 \AA(\sim 12.82 \AA)$. Further, these are indirect bandgap semiconductors (Figure 6.8) irrespective of the core's thickness. For small (big) core nanowire, the PBE functional gives an energy gap of $\sim 1.03 \mathrm{eV}(\sim 0.86 \mathrm{eV})$ with the $\mathrm{CB}$ minimum lying at the $\mathrm{X}$ point and VB maximum lying in between the $\Gamma$ and $\mathrm{X}$ point. Both the VB and $\mathrm{CB}$ in the $\langle 200\rangle$ direction show dispersion with the dispersion width $\sim 0.35 \mathrm{eV}$ and $\sim 0.43 \mathrm{eV}$, respectively for the small core nanowire. For the big core nanowire, we obtain dispersion width of $\sim 0.28 \mathrm{eV}(\sim 0.63 \mathrm{eV})$ for VB (CB). Figure 6.8 also shows that the $\mathrm{CB}$ of this system is mostly $\mathrm{Pb}$ (atoms) with some $\mathrm{S}$ (atoms); the $\mathrm{VB}$ is dominated by both $\mathrm{Pb}$ and $\mathrm{Te}$ atoms irrespective of the core diameter. We further observe that the contribution of Te atoms to inner bands (Figure 6.8(f)) increases with increase in the core's thickness (more Te atoms). With the inclusion of the SOC to our calculations (Figures 6.8(d) and 6.8(h)), the minimum of the $\mathrm{CB}$ of these heterostructures lowers without appreciable change in position of the VB (maximum), indicating the lowering of the energy gap is due to lowering of the $\mathrm{CB}$ in these nanowires. The magnitude of such lowering of the $\mathrm{CB}$ is $\sim 0.20 \mathrm{eV}(\sim 0.30 \mathrm{eV})$ for small (big) core nanowire heterostructures. Since the $\mathrm{CB}$ of this system is mostly $\mathrm{Pb}$, such lowering of $\mathrm{CB}$ is due to the strong SOC effect that arises in the presence the $\mathrm{Pb}$ atoms in these heterostructures. We obtain an energy gap $\sim 0.77 \mathrm{eV}(\sim 0.55 \mathrm{eV})$ for the small (big) core nanowire with the incorporation of spin-orbit interactions. Another noticeable effect of the SOC is to shift the maximum 
(a)

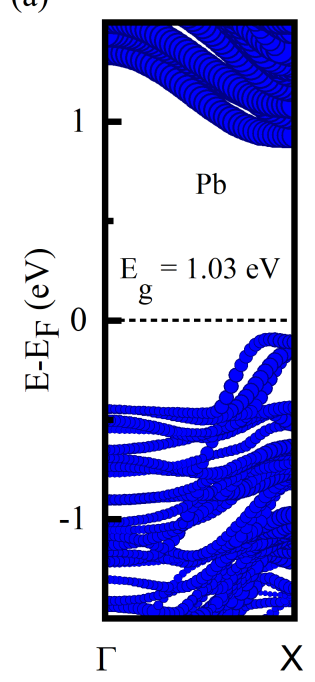

(b)

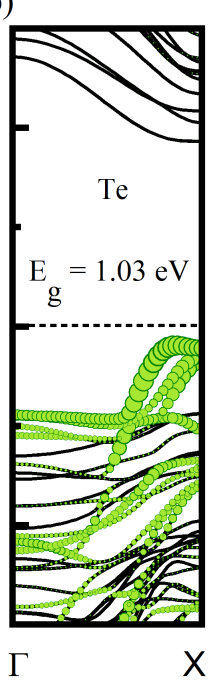

(c)

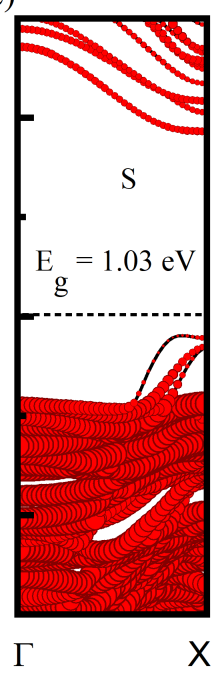

(d)

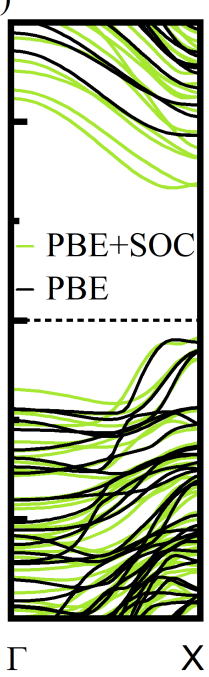

(h)

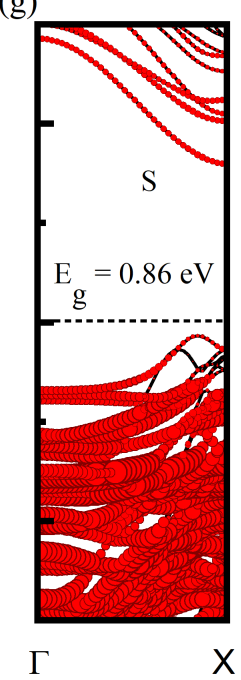

(g)

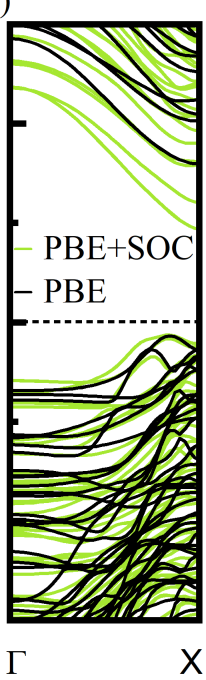

(f)

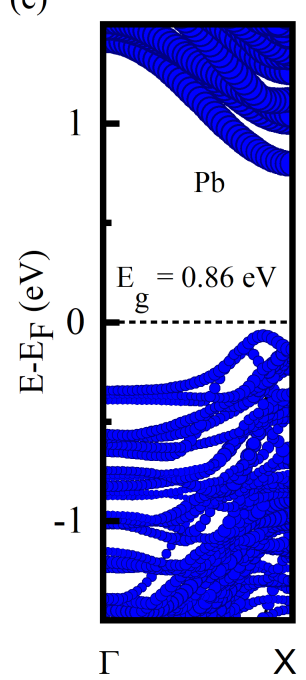

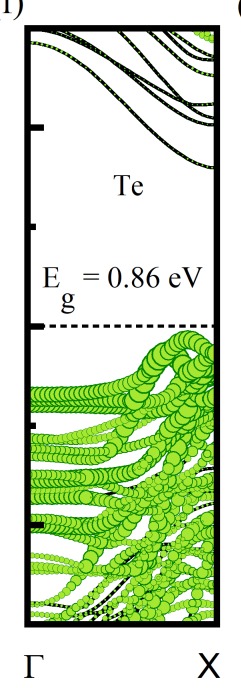

Figure 6.8: The atom decomposed electronic band structure of $\mathrm{PbTe} / \mathrm{PbS}$ core/shell nanowires in the $\langle 200\rangle$ direction for (a-c) small core $\left(d_{c} \sim 6.32 \AA\right)$ (e-f) big core $\left(d_{c} \sim 12.82 \AA\right)$ nanowire heterostructures; the circle represents the contribution of the $\mathrm{Pb}, \mathrm{Te}$, and $\mathrm{S}$ atoms to energy bands. The electronic band structures for small core and big core nanowire obtained using PBE and $\mathrm{PBE}+\mathrm{SOC}$ are compared in $(\mathrm{d})$ and $(\mathrm{h})$. (Reprinted with permission from J. Phys. Chem. C - https://doi.org/10.1021/acs.jpcc.1c06577. Copyright (2021) American Chemical Society.) 
of $\mathrm{VB}$ to $\mathrm{X}$ point; the minimum of the $\mathrm{CB}$ moves in between the $\Gamma$ and $\mathrm{X}$ point. However, SOC does not change the dispersion widths of the VB and CB significantly; the dispersion width obtained using PBE and PBE+SOC agrees well within $0.1 \mathrm{eV}$.

In the $\langle 111\rangle$ direction, the small (big) core nanowire has core diameter of $\sim 5.13$ $\AA(\sim 10.83 \AA)$. The electronic band structure (Figure 6.9) shows that $\mathrm{PbTe} / \mathrm{PbS}$ nanowire is a direct bandgap semiconductor irrespective of the core diameter; the small (big) core nanowire has bandgap of $\sim 0.67 \mathrm{eV}(\sim 1.08 \mathrm{eV})$ in the absence of SOC. Figure 6.9 also reveals that the VB in the $\langle 111\rangle$ direction is almost flat; the CB, however, shows strong dispersion. The dispersion width of $\sim 0.13 \mathrm{eV}(\sim 0.42 \mathrm{eV})$ is obtained for the VB (CB) for wire with small diameter. For the big core nanowire, the dispersion width for the $\mathrm{VB}(\mathrm{CB})$ is $\sim 0.04 \mathrm{eV}(\sim 0.24 \mathrm{eV})$. The dispersion width of the VB estimated here is comparatively smaller than that obtained for nanowires along the $\langle 200\rangle$ direction. Figure 6.9 also reveals that the VB of this system is dominated by $\mathrm{Pb}$, Te, and $\mathrm{S}$ atoms for wires with both the core sizes considered here. However, the contribution to the CB for the small core nanowire comes mostly from $\mathrm{Pb}$ atoms with some $\mathrm{S}$ (atoms). For the big core nanowire, the contribution to the $\mathrm{CB}$ comes significantly from $\mathrm{Pb}$ atoms with some $\mathrm{Te}$ (atoms). Like in the case of the $\mathrm{PbTe} / \mathrm{PbS}$ core/shell nanowire along the $\langle 200\rangle$ direction, the effect of SOC is to move the minimum of $\mathrm{CB}$ (mostly $\mathrm{Pb}$ ) of these heterostructures towards the Fermi level. The magnitude of such lowering is $\sim 0.31 \mathrm{eV}(\sim 0.43 \mathrm{eV})$ for the small (big) core nanowires at the X-point. We obtain the energy gap of $\sim 0.39 \mathrm{eV}(\sim 0.63 \mathrm{eV})$ for 
(a)

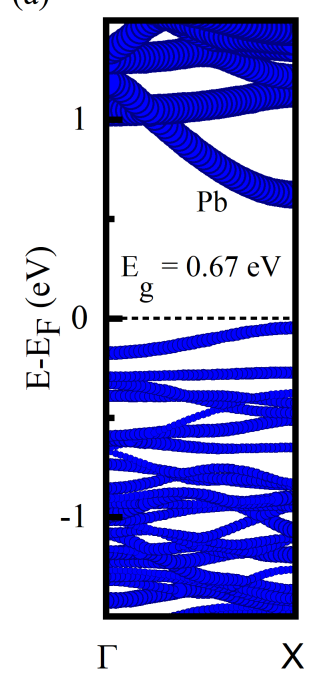

(b)

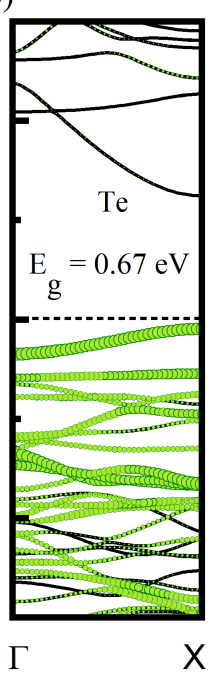

(c)

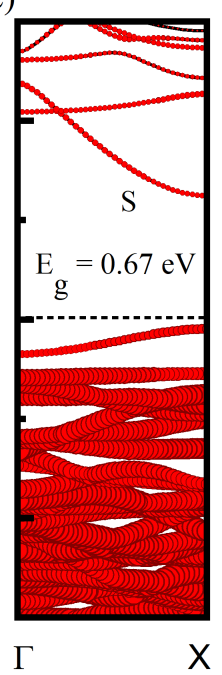

(d)

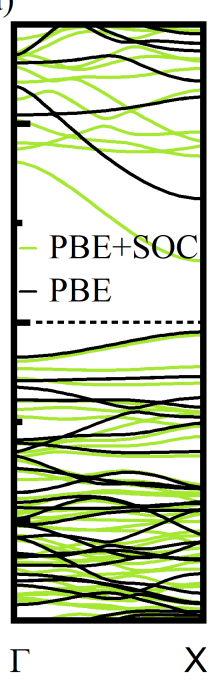

(h)

(g)

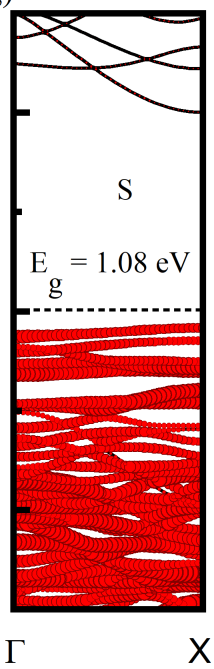

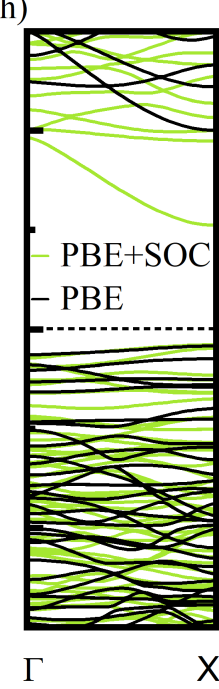

(f)

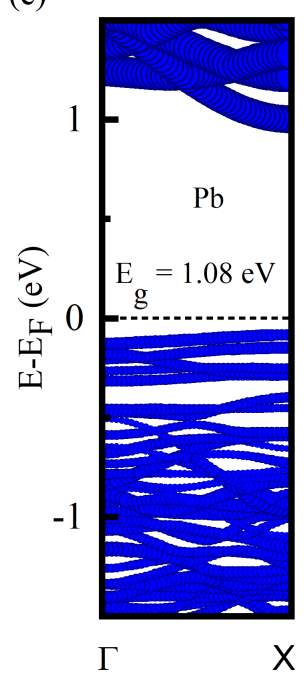

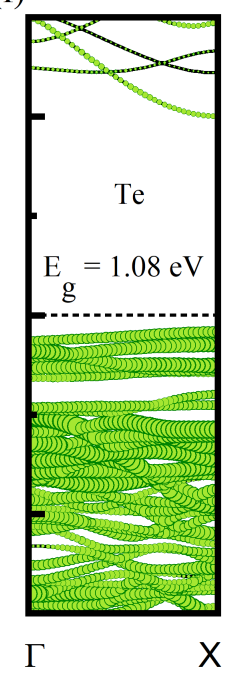

Figure 6.9: The atom decomposed electronic band structure of $\mathrm{PbTe} / \mathrm{PbS}$ core/shell nanowires in the $\langle 111\rangle$ direction for (a-c) small core $\left(d_{c} \sim 5.13 \AA\right)$ (e-f) big core $\left(d_{c} \sim 10.83 \AA\right)$ nanowire heterostructures; the circle represents the contribution of the $\mathrm{Pb}, \mathrm{Te}$, and $\mathrm{S}$ atoms to energy bands. The electronic band structures for small core and big core nanowire obtained using PBE and $\mathrm{PBE}+\mathrm{SOC}$ are compared in $(\mathrm{d})$ and $(\mathrm{h})$. (Reprinted with permission from J. Phys. Chem. C - https://doi.org/10.1021/acs.jpcc.1c06577. Copyright (2021) American Chemical Society.) 
the small (big) core nanowire in the presence of SOC. The SOC does not shift the VB of this system appreciably as expected. For the small core nanowire, the dispersion width of the $\mathrm{VB}$ and $\mathrm{CB}$ with and without SOC agree well within $0.1 \mathrm{eV}$. However, for the big core nanowire, the SOC induced splitting of the energy bands at points of degeneracies increases the dispersion width of the $\mathrm{CB}$ from $0.24 \mathrm{eV}$ to $0.43 \mathrm{eV}$. A comparison of the dispersion width of the VB for big core nanowire with and without SOC shows that they agree fairly well within $0.03 \mathrm{eV}$.

There are several techniques to synthesize lead chalcogenide (core/shell) nanowires, with the two most common being chemical vapor transport (CVT) [72] and solution phase synthesis [191]. Such synthesis processes involve heating/cooling that gives rise to strains. To understand the effect of the strain in these core/shell heterostructures and possible phase transitions at higher values of strain, we calculated the bandgap and relative energy $\left(E=E_{\text {strain }}-E_{0}\right)$ at different values of the tensile $(\epsilon>0)$ and compressive $(\epsilon<0)$ strains, where, $E_{\text {strain }}$ and $E_{0}$ are the energy of the nanowire with and without strain. The results of our calculations are presented in Figure 6.10. To apply strains in these nanowires, we varied the lattice parameter from the equilibrium lattice constant $\left(a_{0}\right)$ and allowed the system to relax. The strain (\%) was then calculated using the relation $\epsilon=\frac{\mathrm{a}-\mathrm{a}_{0}}{\mathrm{a}_{0}} \times 100 \%$, where a is the strained lattice parameter. From Figures 6.10(a) and 6.10(c), we see that the bandgap of the $\mathrm{PbTe} / \mathrm{PbS}$ core/shell nanowire decreases with an increase in the magnitude of the compressive strain irrespective of the core thickness along both the $\langle 200\rangle$ and $\langle 111\rangle$ directions. 
(a)

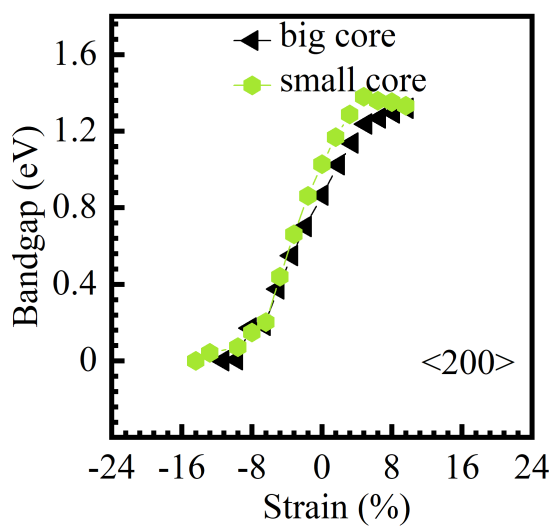

(c)

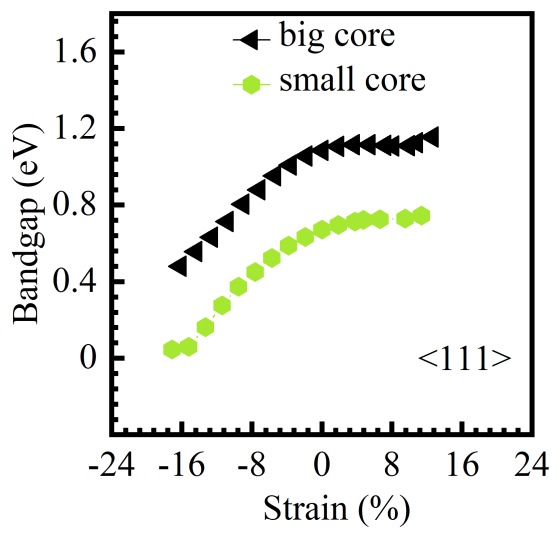

(b)

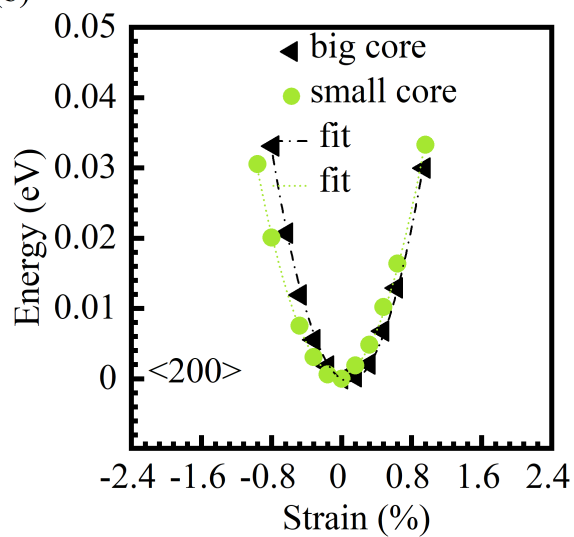

(d)

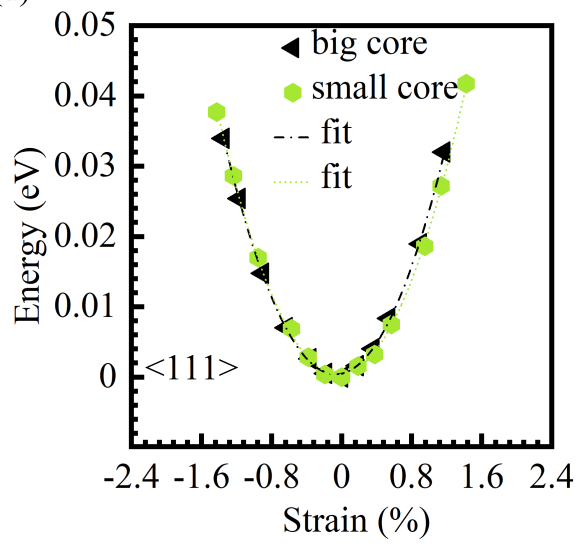

Figure 6.10: (a) Bandgap vs. Strain (\%) (b) Relative energy vs. Strain (\%) plots for $\mathrm{PbTe} / \mathrm{PbS}$ core/shell nanowires along the $\langle 200\rangle$ direction; (c) Bandgap vs. Strain (\%) (d) Relative energy vs. Strain (\%) plots for these heterostructures in the $\langle 111\rangle$ direction. Here, Relative energy $(E)=$ $E_{\text {strain }}-E_{0}$, where $E_{\text {strain }}$ and $E_{0}$ are the energy of the strained and strain free nanowire. The strain (\%) is defined as: $\epsilon(\%)=\left(\frac{a-a_{0}}{a_{0}}\right) \times 100 \%$, where $a_{0}$ is the lattice constant for the strain free nanowire. (Reprinted with permission from J. Phys. Chem. C - https://doi.org/10.1021/acs.jpcc.1c06577. Copyright (2021) American Chemical Society.)

For the nanowires in the $\langle 200\rangle$ direction, the small (big) nanowire undergo semiconductor to metallic phase transition at a strain of $-14.40 \%(-11.30 \%)$. Similarly, in the $\langle 111\rangle$ direction, the semiconductor to metallic phase transition in the small core nanowire occurs at a strain of $-17.1 \%$; the semiconducting feature of the big core 
nanowire remains intact at $-16.0 \%$. With tensile strain, the energy gap of the wires in the $\langle 200\rangle$ direction will increase below $4.8 \%$. Beyond it (4.8\%), there is a relatively low effect of strain on the bandgap. Figure 6.10(c) also shows the small effect of tensile strain on the energy gap for wires long the $\langle 111\rangle$ direction. In the presence of the SOC (Figure 6.11), the compressive strain values at which such phase transition occur are lowered in magnitude without changing much the feature of the bandgap vs. strain curve. Similarly, Figures 6.10(b) and 6.10(d) shows the variation of the relative en-
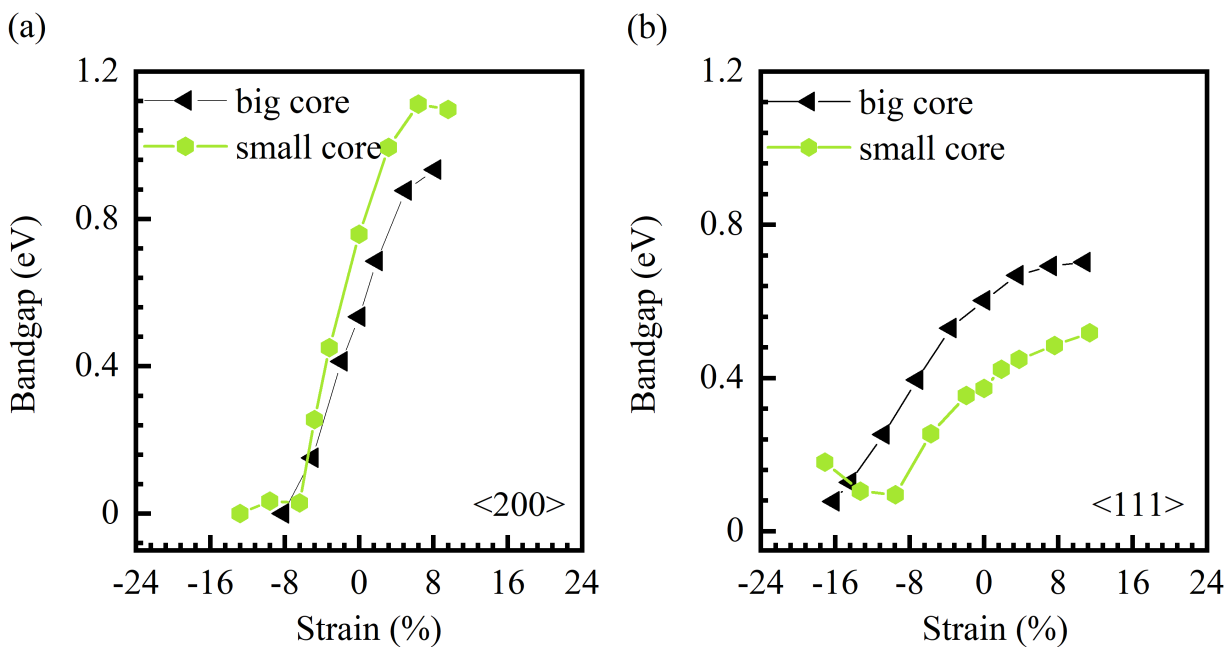

Figure 6.11: Bandgap vs. Strain (\%) plot for $\mathrm{PbTe} / \mathrm{PbS}$ core/shell nanowires in (a) $\langle 200\rangle$ (b) $\langle 111\rangle$ direction. SOC is included in these calculations. (Reprinted with permission from J. Phys. Chem. C - https://doi.org/10.1021/acs.jpcc.1c06577. Copyright (2021) American Chemical Society.)

ergy with the strain (\%). At small values of the strain $(-1.6 \%<\epsilon(\%)<1.6 \%)$, the variation is quadratic which can be fitted to obtain the Young's modulus (Y) using [202]: $\mathrm{Y}=\frac{1}{\mathrm{~V}_{0}} \frac{\partial^{2} \mathrm{E}}{\partial \epsilon^{2}}$. Here, $\frac{\partial^{2} \mathrm{E}}{\partial \epsilon^{2}}$ is the coefficient obtained by the parabolic fit of the 
relative energy $\left(E=E_{\text {strain }}-E_{0}\right)$ vs strain curve and $\mathrm{V}_{0}$ is the volume of the strain free nanowire. Our calculations give Y values of $41.25 \mathrm{GPa}(47.35 \mathrm{GPa})$ for the small (big) core nanowire in the $\langle 200\rangle$ direction. The estimated $\mathrm{Y}$ value for small (big) core nanowires in the $\langle 111\rangle$ direction is $20.51 \mathrm{GPa}(19.52 \mathrm{GPa})$. Our study further reveals that $\mathrm{Y}$ value of the nanowire does not change much with the inclusion of the SOC in our calculations. With SOC, Y value for the small (big) core nanowire is 44.28 $\mathrm{GPa}(42.78 \mathrm{GPa})$ along the $\langle 200\rangle$ direction and $19.89 \mathrm{GPa}(17.18 \mathrm{GPa})$ in the $\langle 111\rangle$ direction. Our calculated values of $\mathrm{Y}$ are in a very good agreement with previously reported Y values for bulk Te and Te nanowires 202]. Subsequently, we estimated the pressure required for phase transitions using the relation: $\mathrm{P}=\mathrm{Y} \times\left(\frac{\mathrm{a}-\mathrm{a}_{0}}{\mathrm{a}_{0}}\right)$. In the $\langle 200\rangle$ direction (without SOC), the small (big) core nanowire undergo a phase transition at a pressure of $\sim 5.94 \mathrm{GPa}(5.35 \mathrm{GPa})$; the corresponding pressure in the $\langle 111\rangle$ direction for small core nanowire is $\sim 3.50 \mathrm{GPa}$. With SOC, we get $\mathrm{P} \sim 5.67$ GPa (3.49 GPa) for small (big) core nanowire along the $\langle 200\rangle$ direction and $\sim 1.89$ $\mathrm{GPa}(2.78 \mathrm{GPa})$ for small (big) core nanowire in the $\langle 111\rangle$ direction. It may be noted that the pressure for phase transition obtained for the nanowires (both with and without SOC) along the $\langle 200\rangle$ direction agree well with the reported pressure of 3.1 $\mathrm{GPa}$ [75] for PbS nanowires and $6 \mathrm{GPa}[203]$ for the PbS nanoparticles. In the $\langle 111\rangle$ direction, PBE gives a good estimate of the pressure for phase transition in these heterostructures.

Next, we shift our discussion to the optical properties (Figure 6.12) of $\mathrm{PbTe} / \mathrm{PbS}$ 
nanowires in the $\langle 200\rangle$ direction. We first discuss the optical spectra in the absence of the SOC. Figure 6.12 reveals the anisotropic behavior of the real $\left(\epsilon_{1}(\omega)\right)$ and
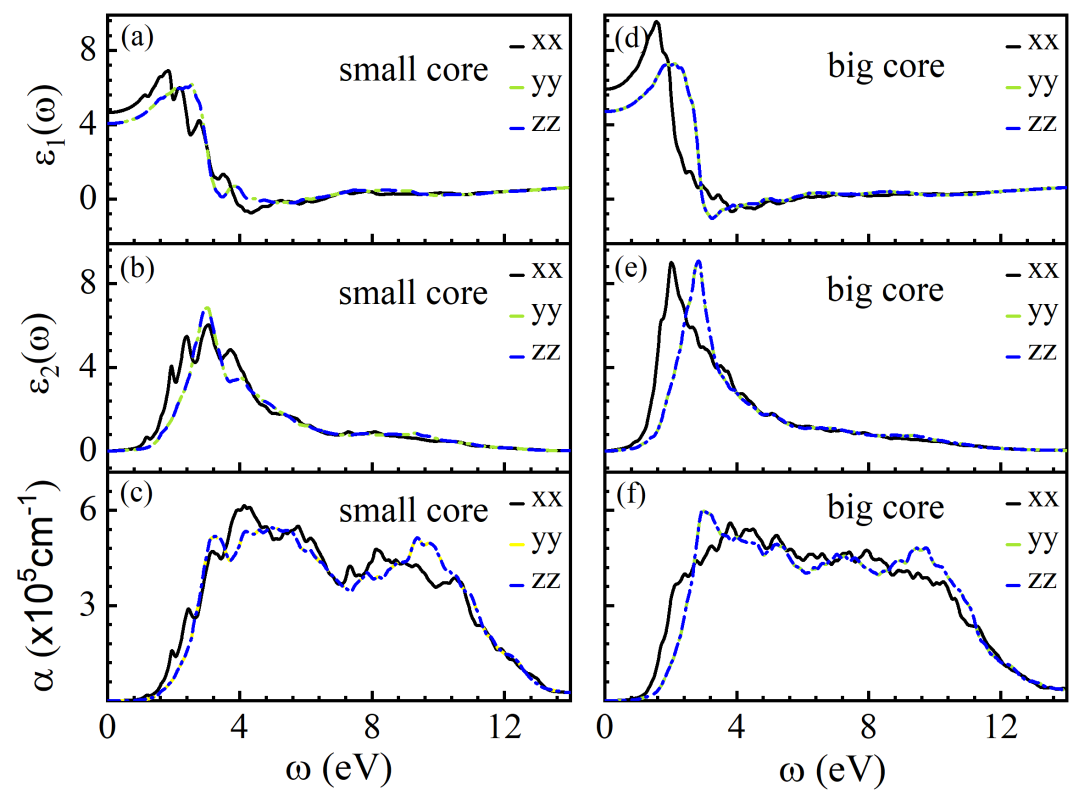

Figure 6.12: The energy dependence of the real $\left(\epsilon_{1}(\omega)\right)$ part of $\epsilon(\omega)$, imaginary $\left(\epsilon_{2}(\omega)\right)$ part of $\epsilon(\omega)$, and absorption coefficient $(\alpha)$ for (a-c) small core (d-f) big core nanowire in the $\langle 200\rangle$ direction. No SOC is included in these calculations. (Reprinted with permission from J. Phys. Chem. C - https://doi.org/10.1021/acs.jpcc.1c06577. Copyright (2021) American Chemical Society.)

imaginary $\left(\epsilon_{2}(\omega)\right)$ part of the complex dielectric function $\left(\epsilon(\omega)=\epsilon_{1}(\omega)+i \epsilon_{2}(\omega)\right)$, as well as the absorption coefficient $\alpha(\omega)$; their yy and zz components are identical and are different from the $\mathrm{xx}$ components. This is evident due to symmetric nature of the wire in the $\mathrm{y}$ - and z-direction, but not in $\mathrm{x}$-direction-the nanowire axis. From the real part of the dielectric function (Figure 6.12(a) and 6.12(d)), we get the static dielectric constant values of $\epsilon^{x x}(0)=4.65(5.91), \epsilon^{y y}(0)=4.06(4.71)$, and $\epsilon^{z z}(0)=4.06(4.71)$ 

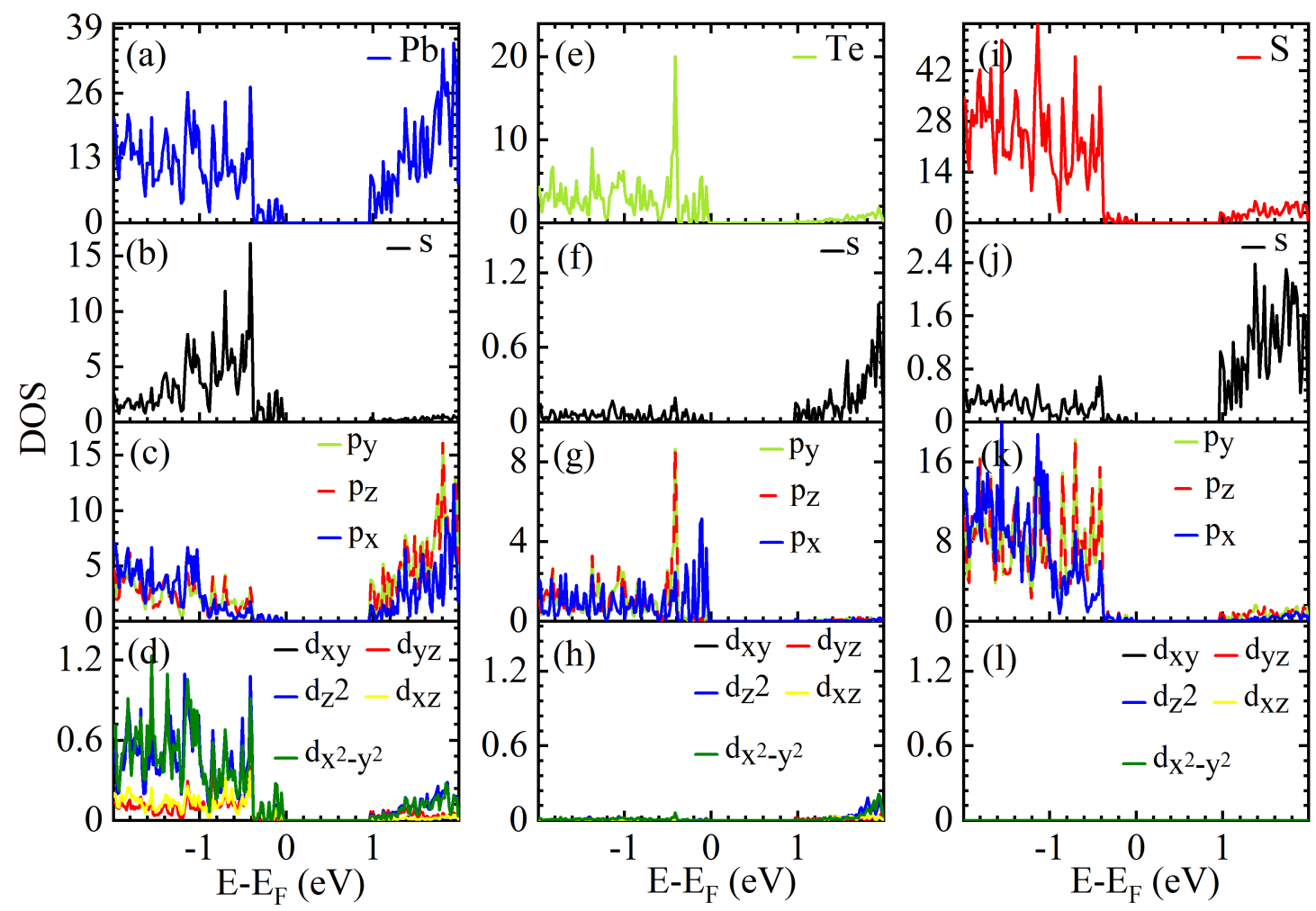

Figure 6.13: Atom and orbital decomposed electronic density of states (DOS) of small core $\mathrm{PbTe} / \mathrm{PbS}$ nanowire in the $\langle 200\rangle$ direction. No SOC is included in these calculations. (Reprinted with permission from J. Phys. Chem. C - https://doi.org/10.1021/acs.jpcc.1c06577. Copyright (2021) American Chemical Society.)

for the small (big) core nanowires. Consequently, we obtain the static refractive index of this material using $n^{i i}(0)=\sqrt{\epsilon_{1}^{i i}(0)}$, where $\mathrm{i}=\{\mathrm{x}, \mathrm{y}, \mathrm{z}\}$. Our calculations give $n^{x x}(0)$ $=2.16(2.43), n^{y y}(0)=2.01(2.17)$, and $n^{z z}(0)=2.01$ (2.17) for the small (big) core nanowire. Figures 6.12(b) and 6.12(e) show the imaginary part $\epsilon_{2}^{i i}(\omega), i=x, y, z$ of the complex dielectric function for the small and big core nanowires. These contain several features that peak at $3.05 \mathrm{eV}$ and $3.02 \mathrm{eV}$ for small core nanowire and at $2.01 \mathrm{eV}$ and $2.85 \mathrm{eV}$ for the big core nanowire. We also notice that the peaks in 

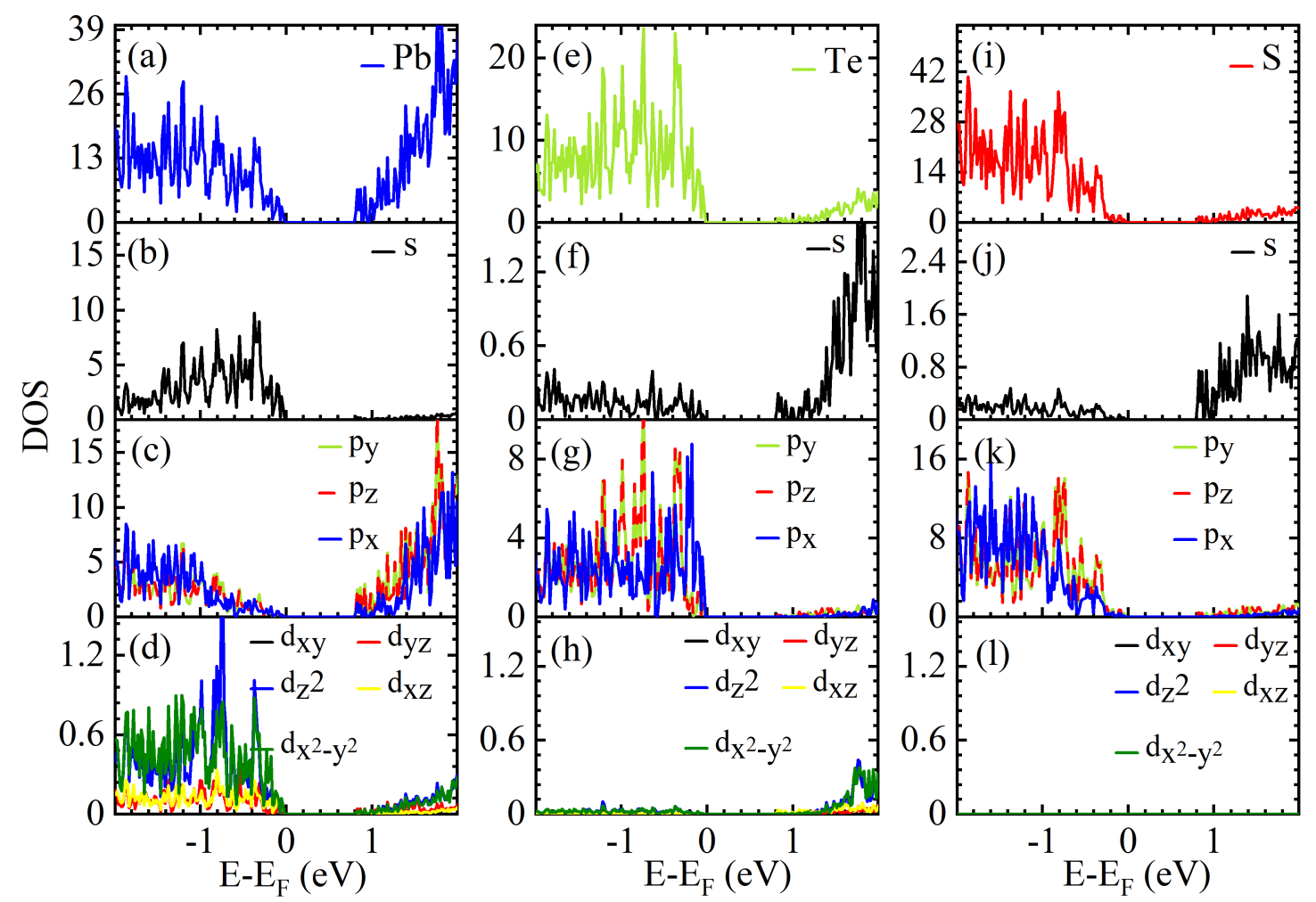

Figure 6.14: Atom and orbital decomposed electronic density of states (DOS) of big core $\mathrm{PbTe} / \mathrm{PbS}$ nanowire in the $\langle 200\rangle$ direction. No SOC is included in these calculations. (Reprinted with permission from J. Phys. Chem. C - https://doi.org/10.1021/acs.jpcc.1c06577. Copyright (2021) American Chemical Society.)

$\epsilon_{2}^{i i}(\omega), i=x, y, z$ are well separated on increasing the core thickness. Finally, we obtain the $\mathrm{xx}\left(\alpha^{x x}(\omega)\right)$, yy $\left(\alpha^{y y}(\omega)\right)$ and $\mathrm{zz}\left(\alpha^{z z}(\omega)\right)$ components of absorption using $\alpha(\omega)=\frac{4 \pi k}{\lambda}=\frac{4 \pi}{\lambda} \sqrt{\frac{\left(\epsilon_{1}^{2}+\epsilon_{2}^{2}\right)^{\frac{1}{2}}-\epsilon_{1}}{2}}$; the results are shown in Figures 6.12(c) and 6.12(f). Like the components of the real and imaginary parts of the dielectric function, the components of the absorption show different features that have origin in the interband electronic transitions from the VB to CB. The absorption spectra for the small (big) core nanowire has interesting feature at $1.18 \mathrm{eV}(0.93 \mathrm{eV})$ which is approximately 

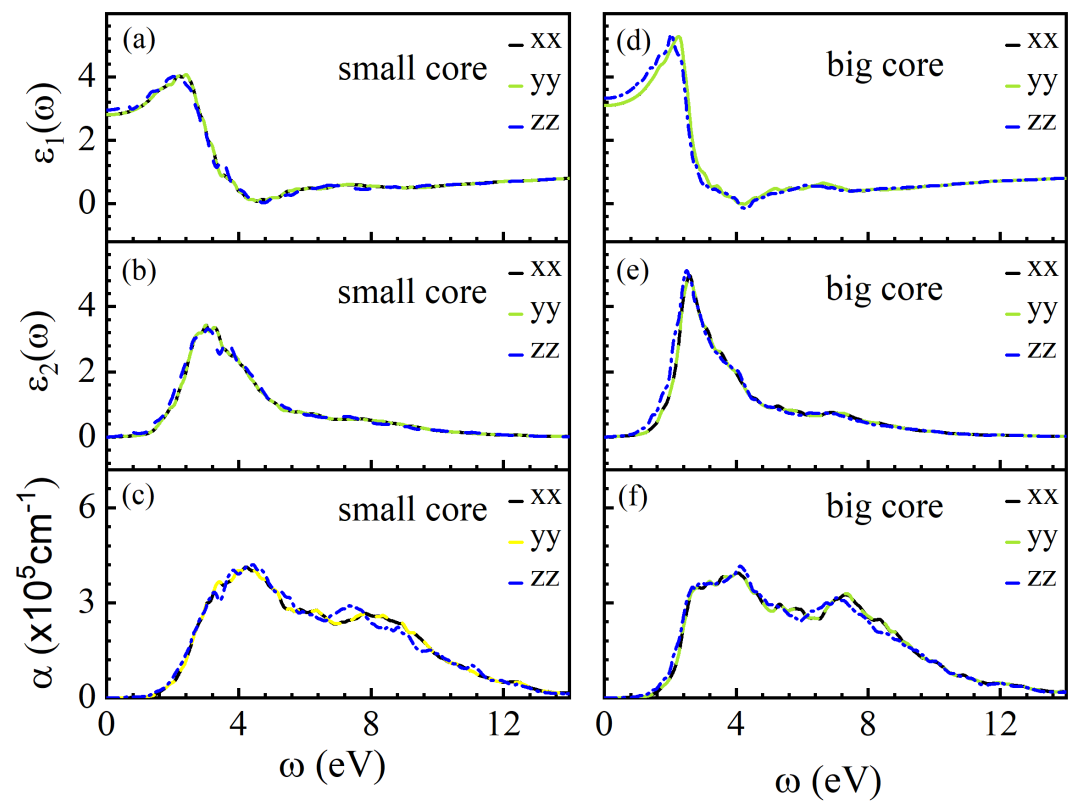

Figure 6.15: The energy dependence of the real $\left(\epsilon_{1}(\omega)\right)$ part of $\epsilon(\omega)$, imaginary $\left(\epsilon_{2}(\omega)\right)$ part of $\epsilon(\omega)$, and absorption coefficient $(\alpha)$ for (a-c) small core (d-f) big core nanowire in the $\langle 111\rangle$ direction. No SOC is included in these calculations. (Reprinted with permission from J. Phys. Chem. C - https://doi.org/10.1021/acs.jpcc.1c06577. Copyright (2021) American Chemical Society.)

the bandgap obtained for these materials in the absence of SOC. These peaks are approximately the onset of the absorption in these materials and correspond to the electronic transition from the VB to CB. To see possibilities of such transitions, we have calculated the density of states (DOS) of these materials; the results are shown in Figures 6.13 and 6.14. DOS calculations show that the contribution to the VB comes mostly from the s and $\mathrm{p}$ orbitals of $\mathrm{Pb}$ and $\mathrm{p}$ orbitals of $\mathrm{Te}$; the contribution to the $\mathrm{CB}$ comes mostly from the p orbitals of $\mathrm{Pb}$ and s orbitals of Te and $\mathrm{S}$, suggesting the possibility of $\mathrm{s}$ to $\mathrm{p}$ or $\mathrm{p}$ to $\mathrm{s}$ transitions in these materials when they are excited by light. We also see that the absorption in this material extends from the infrared to 

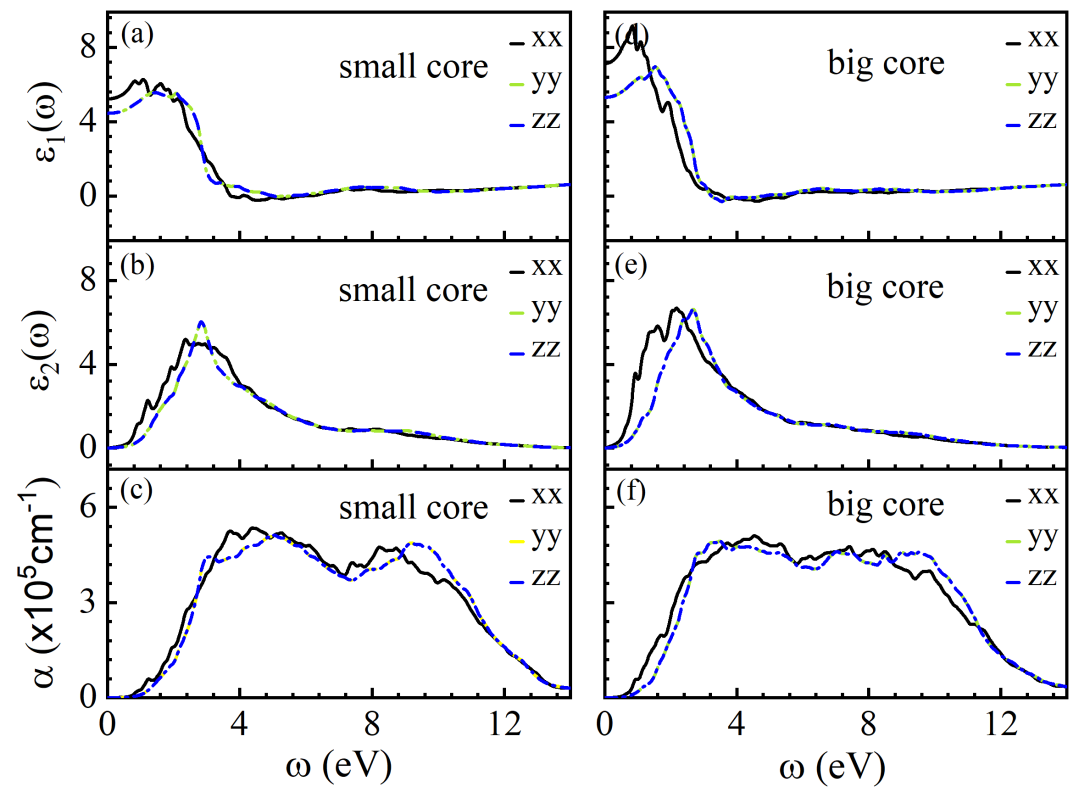

Figure 6.16: The energy dependence of the real $\left(\epsilon_{1}(\omega)\right)$ part of $\epsilon(\omega)$, imaginary $\left(\epsilon_{2}(\omega)\right)$ part of $\epsilon(\omega)$, and absorption coefficient $(\alpha)$ for (a-c) small core (d-f) big core nanowire in the $\langle 200\rangle$ direction. SOC is included in these calculations. (Reprinted with permission from J. Phys. Chem. C - https://doi.org/10.1021/acs.jpcc.1c06577. Copyright (2021) American Chemical Society.)

visible to the ultraviolet region $(\sim 1.0 \mathrm{eV}$ to $13 \mathrm{eV})$, suggesting the suitability of these materials for optoelectronic applications. Finite absorption in the ultraviolet region is also indicative of the fact that there can be the transitions of the electrons from deep levels in the VB to CB as DOS is negligible beyond $3.37 \mathrm{eV}$. Figure 6.15 shows that the $\mathrm{xx}, \mathrm{yy}$, and $\mathrm{zz}$ components of the real and imaginary part of the dielectric function, as well as the absorption, are identical for the wires in the $\langle 111\rangle$ direction. It may be noted that the periodicity of wires in the $\langle 111\rangle$ direction are in the $\mathrm{z}$-direction unlike the nanowires in $\langle 200\rangle$ direction that are periodic in $\mathrm{x}$-direction. Comparison of the optical spectra of these wires in the two directions in the absence of SOC shows 

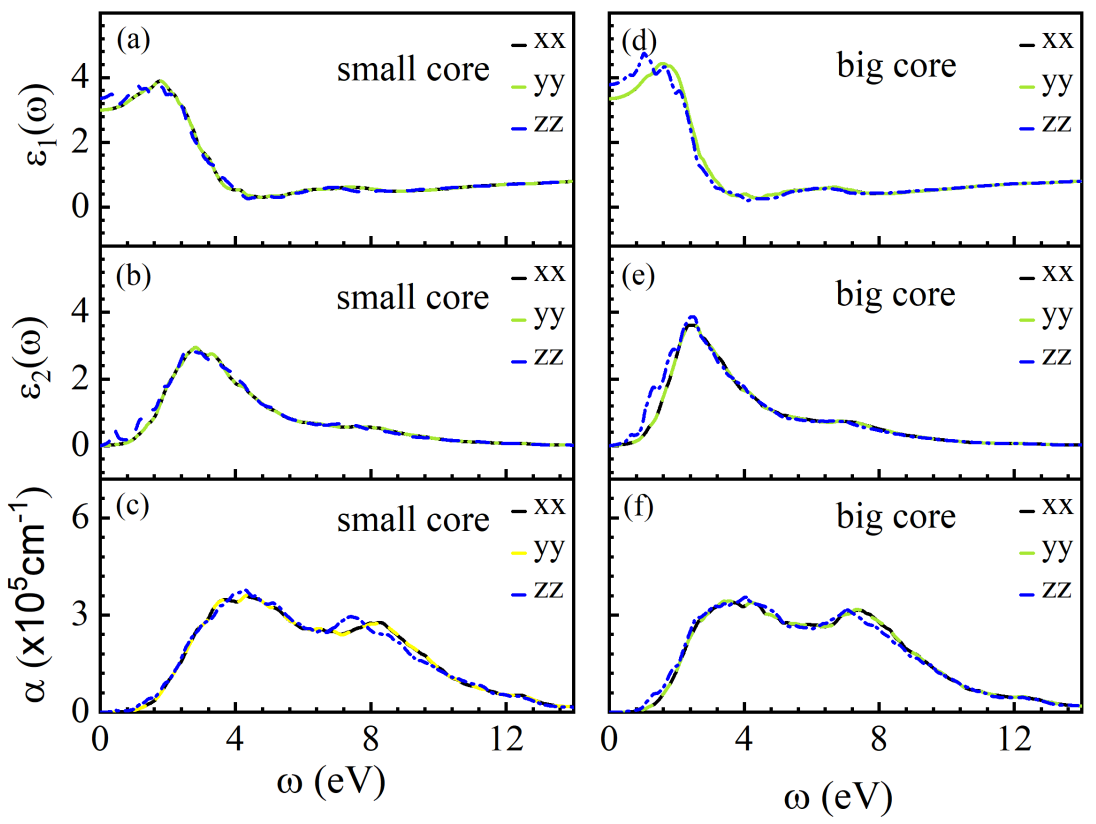

Figure 6.17: The energy dependence of the real $\left(\epsilon_{1}(\omega)\right)$ part of $\epsilon(\omega)$, imaginary $\left(\epsilon_{2}(\omega)\right)$ part of $\epsilon(\omega)$, and absorption coefficient $(\alpha)$ for (a-c) small core $(\mathrm{d}-\mathrm{f})$ big core nanowire in the $\langle 111\rangle$ direction. SOC is included in these calculations. (Reprinted with permission from J. Phys. Chem. C - https://doi.org/10.1021/acs.jpcc.1c06577. Copyright (2021) American Chemical Society.)

that the magnitude of the absorption coefficient for wires along the $\langle 111\rangle$ decreases by $32 \%$ compared to that in the $\langle 200\rangle$ direction. The absorption spectra, however, remains broad $(0.67 \mathrm{eV}$ to $13 \mathrm{eV})$. The static refractive index values of $n^{x x}(0)=1.67$ (1.75), $n^{y y}(0)=1.67(1.75)$, and $n^{z z}(0)=1.71$ (1.82) are obtained for the small (big) core nanowire. Compared to the values obtained for nanowires in the $\langle 200\rangle$ direction, the values of refractive index decrease by approximately $\sim 21 \%$. The main effect of the SOC (Figures 6.16 and 6.17) to the absorption spectra is to redshift the entire absorption curve without changing the overall features of the absorption. The redshift is expected as the SOC will decrease the bandgap of these materials. 
We now study the stability of these nanowire heterostructures along the $\langle 200\rangle$ and $\langle 111\rangle$ directions. For this, we begin by calculating the cohesive energy per atom using $E_{c}=\left[E_{n w}-\sum_{i} n_{i} E_{i}\right] / n_{\text {atoms }}$, where i may be $\mathrm{Pb}$, Te, S. $n_{i}, n_{\text {atoms }}, E_{i}$, and $E_{n w}$ are the the number of atoms of type i, total number of atoms in the nanowire, energy of an isolated atom i, and energy of the nanowire respectively. The estimated value of

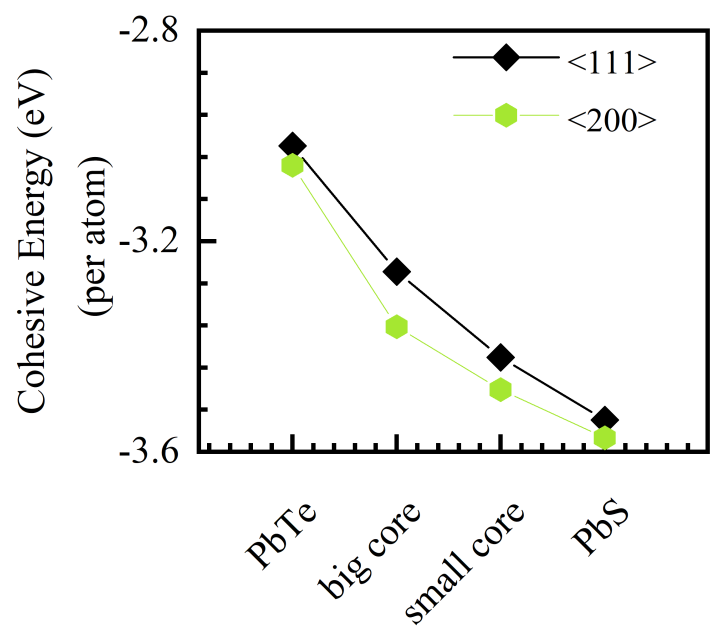

Figure 6.18: Cohesive energy of the pristine and core/shell nanowires along the $\langle 200\rangle$ and $\langle 111\rangle$ directions; the stability of nanowires in the $\langle 111\rangle$ direction is relatively less compared to that in $\langle 200\rangle$ direction. (Reprinted with permission from J. Phys. Chem. C - https://doi.org/10.1021/acs.jpcc.1c06577. Copyright (2021) American Chemical Society.)

the cohesive energy for small core nanowire along the $\langle 200\rangle(\langle 111\rangle)$ direction is -3.48 $\mathrm{eV}(-3.42 \mathrm{eV})$. For the big core nanowire, the cohesive energy is $-3.36 \mathrm{eV}(-3.26 \mathrm{eV})$ in the $\langle 200\rangle(\langle 111\rangle)$ direction. This suggests that wires along the $\langle 111\rangle$ are less stable 
compared to the nanowires in the $\langle 200\rangle$ direction. Our calculations (Figure 6.18) further reveal that the stability of core/shell structures lie in between the stability of pristine $\mathrm{PbTe}$ and $\mathrm{PbS}$ nanowires with possible hierarchy being $\mathrm{PbS}>\mathrm{PbTe} / \mathrm{PbS}$ $($ small core $)>\mathrm{PbTe} / \mathrm{PbS}$ (big core) $>\mathrm{PbTe}$. Now one may ask: What happens to the stability of these nanowires with strain? Application of the tensile or compressive strain decreases the stability of these nanowires. For instance, the cohesive energy of a small core nanowire at a strain of $\sim-11.0 \%$ differ by $83 \mathrm{meV}(30 \mathrm{meV})$ from the cohesive energy of strain free nanowire along the $\langle 200\rangle(\langle 111\rangle)$ direction . We now investigate the possibility of the core to shell diffusion of Te atoms in these heterostructures. For this, we performed ab-initio MD simulations for 8 ps at room temperature $(300 \mathrm{~K})$ with a time resolution of $2 \mathrm{fs}$. The results of our simulations are shown in Figure 6.19. In the $\langle 200\rangle$ direction, the temperature (Figure 6.19(a)) and energy (Figure 6.19(c)) fluctuations are small after 0.4 ps. Such (Figure 6.19b \& 6.19d) fluctuations are stabilized after $1.6 \mathrm{ps}$ for wire along the $\langle 111\rangle$ direction. The radial distribution function $(\mathrm{RDF})$ for the nanowire in the $\langle 200\rangle$ direction has peaks at $\sim 3.10 \AA \& \sim 3.19 \AA$, which corresponds to the Pb-Te bond length. On the other hand, the RDF (Figure 6.19) for the wire in the $\langle 111\rangle$ direction shows peaks close to the Pb-Te bond distance $(\sim 3.10 \AA$ and $\sim 3.16 \AA)$, besides the other two peaks at $\sim 5.68 \AA$ and $\sim 6.82 \AA$. These peaks $(\sim 5.68 \AA$ and $\sim 6.82 \AA)$ for the nanowire along the $\langle 111\rangle$ direction are indicative of the fact that there is possibility of the core to shell diffusion of Te atoms in this nanowire; the wire in the $\langle 200\rangle$ direction has 

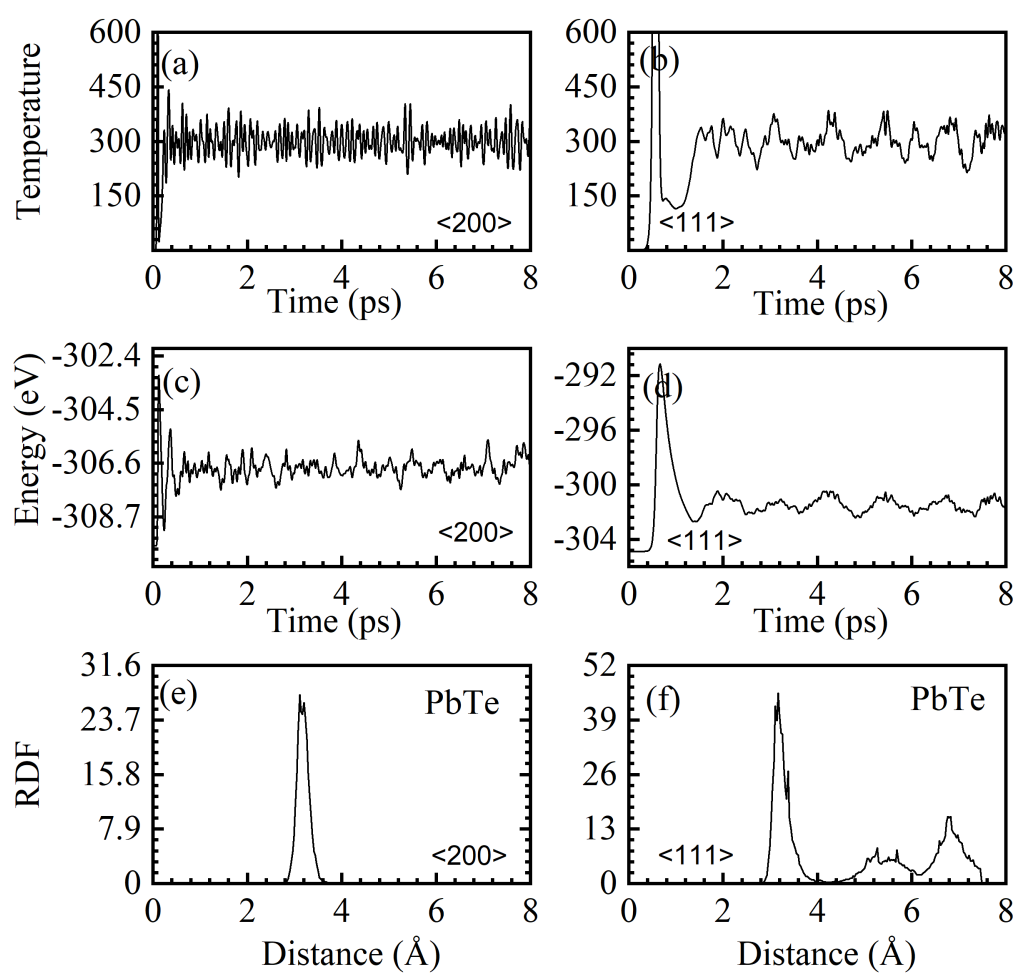

Figure 6.19: Temperature vs. Time $((\mathrm{a}) \&(\mathrm{~b}))$ and Energy vs. Time ((c) \& (d)) plot for small core nanowires oriented in $\langle 200\rangle$ and $\langle 111\rangle$ directions; the partial $(\mathrm{Pb}-\mathrm{Te})$ radial distribution function $(\mathrm{RDF})$ is shown in (e) and (f). (Reprinted with permission from J. Phys. Chem. C - https://doi.org/10.1021/acs.jpcc.1c06577. Copyright (2021) American Chemical Society.)

minimal possibility of such diffusion as seen from its RDF. These analysis allow us to conclude that the core/shell nanowire heterostructures along the $\langle 200\rangle$ direction are more stable compared to that in the $\langle 111\rangle$ direction. Here, we want to mention that our results of the MD run (Figure 6.20) for the big core nanowire are consistent with the small core nanowire. Therefore, irrespective of the core thickness, the wires in the $\langle 111\rangle$ direction are more susceptible to the core to shell diffusion at room temperature. 

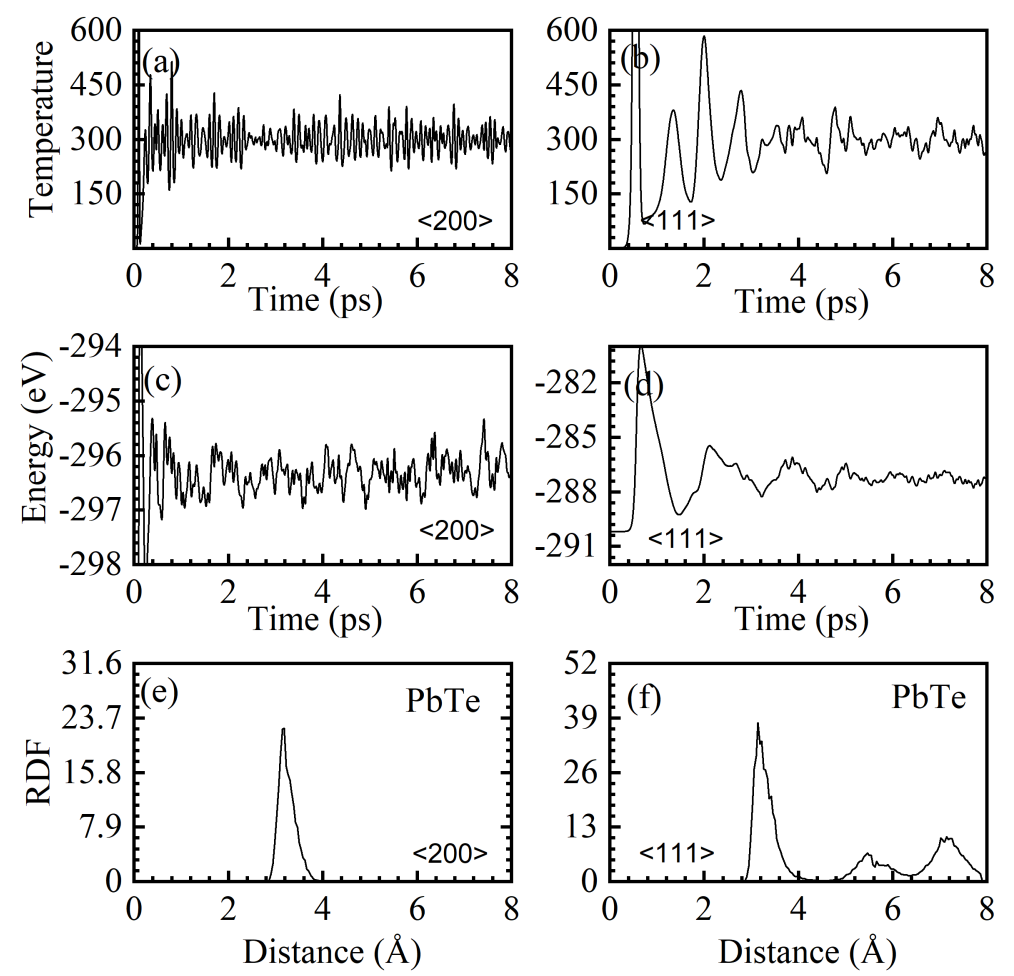

Figure 6.20: Temperature vs. Time $((\mathrm{a}) \&(\mathrm{~b}))$ and Energy vs. Time $((c) \&(d))$ plot for big core nanowires oriented in $\langle 200\rangle$ and $\langle 111\rangle$ directions; the partial ( $\mathrm{Pb}-\mathrm{Te}$ ) radial distribution function $(\mathrm{RDF})$ is shown in (e) and (f). (Reprinted with permission from J. Phys. Chem. C - https://doi.org/10.1021/acs.jpcc.1c06577. Copyright (2021) American Chemical Society.)

\subsection{Conclusion}

In summary, our DFT calculations reveal that $\mathrm{PbTe} / \mathrm{PbS}$ core/shell nanowire is an indirect (direct) bandgap semiconductor in the $\langle 200\rangle(\langle 111\rangle)$ direction; the bandgap depends upon the diameter of the core and shell i.e. the number of the Te atoms in the core and S atoms in the shell. The effect of the SOC on the energy band structure 
is to move the $\mathrm{CB}$, that is dominated by $\mathrm{Pb}$ atoms, towards the Fermi energy. Such lowering is of the order of $0.20 \mathrm{eV}-0.43 \mathrm{eV}$ at the $\mathrm{X}$ point. The estimated value of the Young's modulus is $\sim 48 \mathrm{GPa}(\sim 20 \mathrm{GPa})$ for the wires in the $\langle 200\rangle(\langle 111\rangle)$ direction. These heterostructures undergo semiconductor to metallic phase transitions at high values of compressive strains; the magnitude of the pressure for phase transitions is in good agreement with previously reported experimental values in lead chalcogenides nanowires and nanoparticles. These materials have a broad optical absorption spectrum that extends up to $13 \mathrm{eV}$ and includes the infrared-visible-ultraviolet region. Several peaks in the absorption spectra correspond to the interband electronic transitions from the s to $\mathrm{p}$ or $\mathrm{p}$ to s states. Analysis of the cohesive energy shows that the wires along the $\langle 200\rangle$ direction are more stable compared to wires in the $\langle 111\rangle$ direction; the ab-initio MD calculations reveal that the nanowires in the $\langle 111\rangle$ direction are prone to the core to shell diffusion at room temperature. 


\section{Chapter 7}

\section{Conclusion and Future}

\section{Perspectives}

\subsection{Conclusion}

In this thesis, we studied the properties of core/shell heterostructures, mainly the $\mathrm{Ge} / \mathrm{Si}$ (Mn and $\mathrm{Cr}$ doped) and $\mathrm{PbTe} / \mathrm{PbS}$ (undoped) core/shell nanowires. In the first project (chapter 4 of this thesis), using first-principles density functional theory, we report that a small concentration of Mn dopants $(\leq 2 \%)$ in the Ge-core transform the Ge/Si core/shell nanowire (a direct bandgap semiconductor) to a room temperature stable half-metallic ferromagnetic system. The electronic band structure shows 
that Mn-doped Ge/Si core/shell nanowire is semiconducting in the minority spin direction and metallic in the majority spin direction with $100 \%$ spin polarization at the Fermi level; the half-metallic behavior is also confirmed from the density of states calculations. Here, we also report that the stability of the ferromagnetic ordering between Mn atoms is mediated by the itinerant electrons which are the majority spin carriers in the vicinity of the Fermi level in the metallic channel. Spin-orbit (SO) interactions have negligible effect to the energy bands that are in the proximity of the Fermi energy; SO splittings of $\leq 24 \mathrm{meV}$ have been observed at the crossing points of minority and majority bands away from the Fermi level. This also suggests that the spin coherence length can be much higher in this material. The spin-dependent quantum transport calculations in a prototype nanowire junction give a spin-filtering efficiency of $90.4 \%$, indicating the excellent spin-selective properties of this newly tailored material.

Given that the substitutional doping of Mn into the Ge-core/Si-shell nanowire transforms the semiconducting material to a ferromagnetic half-metal, we explored the possibility of antiferromagnetic (AFM) semiconducting behavior in the second project (chapter 5 of this thesis). Using quantum mechanical DFT calculations, we report the Cr-doped Ge/Si core/shell nanowire as an AFM semiconductor. The origin of the AFM spin ordering between the localized $d_{y z}$ orbitals of $\mathrm{Cr}$ is due to the superexchange mechanism mediated by the $p_{z}$ orbitals of the Ge that are in the neighborhood of the Cr. Like in the case of Mn-doped Ge/Si core/shell nanowire, the effect of the 
SOC to bands near the Fermi level is found to be negligible, suggesting a longer spin coherence length in this material. The spin-polarized quantum transport calculations in a finite segment of Cr-doped nanowire junction shows that the electrical current in the doped nanowire is $\sim 24$ times higher in magnitude than the undoped nanowire at a small bias of $0.83 \mathrm{~V}$. Furthermore, the current has a switching feature with a high $\mathrm{ON} / \mathrm{OFF}$ current ratio $\sim 41$ (at $0.83 \mathrm{~V}$ ). The projected DOS calculations confirm that $\mathrm{Cr}$ in Cr-doped nanowire is responsible for such switching behavior, as well as high current compared to the undoped nanowire.

In the third project (chapter 6 of this thesis), we studied the electronic structure, thermodynamic stability, mechanical and optical properties of $\mathrm{PbTe} / \mathrm{PbS}$ nanowire heterostructures. Using the many-body DFT calculations, we reveal that $\mathrm{PbTe} / \mathrm{PbS}$ core-shell nanowire is a direct bandgap semiconductor along the $\langle 111\rangle$ direction and an indirect bandgap semiconductor in the $\langle 200\rangle$ direction; the energy gap can be tuned by changing the size of the core and shell. Inclusion of the SOC to our calculation lowers the conduction band (mostly $\mathrm{Pb}$ atoms) of these materials towards the Fermi level by $0.20 \mathrm{eV}-0.43 \mathrm{eV}$ at the $\mathrm{X}$ point, reducing the overall energy gap of this material; the SOC induced shift in the valence band is negligible. A semiconductor to the metallic phase transition is observed at high compressive strains $>11.30 \%$. The values of Young's modulus for the wires oriented along the $\langle 111\rangle(\langle 200\rangle)$ direction are $\sim 20 \mathrm{GPa}(\sim 48 \mathrm{GPa})$. We further report that these materials have broad absorption $(0.39 \mathrm{eV}-13 \mathrm{eV})$ that includes the infrared-visible-ultraviolet region; the peaks in the 
absorption represent the interband electronic transitions from the valence band to the conduction band. Analysis of the cohesive energy for these nanowires indicates that wires along the $\langle 200\rangle$ direction are more stable than that in the $\langle 111\rangle$ direction. The radial distribution functions obtained from the ab-initio molecular dynamics simulations at room temperature confirm that nanowires along the $\langle 111\rangle$ direction are prone to core to shell diffusion at room temperature. For wires in the $\langle 200\rangle$ direction, the chance of such diffusion is minimal, suggesting their higher stability.

\subsection{Future Perspectives}

One-dimensional core/shell nanowires, especially $\mathrm{Ge} / \mathrm{Si}$ and $\mathrm{PbTe} / \mathrm{PbS}$ core/shell nanowire heterostructures, offer a wide range of applications including but not limited to high performance field effect transistors (FETs), quantum computing devices, solar

cells, light emitting diodes (LEDs), sensors, logic circuits, lasers, and thermoelectric devices as discussed earlier. Incorporating magnetic impurity ( $\mathrm{Mn} \& \mathrm{Cr}$ ) in a $(\mathrm{Ge} / \mathrm{Si})$ core/shell semiconductor nanowire provides an additional opportunity for its usage in next-generation spintronics. Therefore, in chapter 4 of this thesis, we predicted theoretically that the $\mathrm{Mn}$-doped $\mathrm{Ge} / \mathrm{Si}$ core/shell nanowire is a room temperature stable FM half metal that can be used as an excellent spin filter. Similarly, in chapter 5, we reported that the Cr-doped Ge/Si core/shell nanowire is an AFM semiconductor that can be used as a switch. In the future, we expect these unique predictions to 
initiate experimental studies in these materials, due to the compatibility of the Ge and Si with current Si-based technology, for potential applications in low dimensional FM/AFM spintronics.

In chapter 6 , we studied the electronic structure, thermodynamic stability, mechanical and optical properties of $\mathrm{PbTe} / \mathrm{PbS}$ nanowires. In the future, we would like to study the charge transport in this material to explore the transistor behavior for possible applications in next-generation nanoelectronics. We would also like to explore the thermoelectric properties of these heterostructures for different core diameters. 



\section{References}

[1] Waldrop, M. Nature News 2016, 530(7589), 144.

[2] Carroll, R.; Gorman, C. Angew. Chem. Int. Ed. 2002, 41(23), 4378-4400.

[3] Moore, G. Electronics 1965, 38(8), 114-117.

[4] Moore, G. IEDM Tech. Digest 1975, pages pp. 11-13.

[5] Chau, R.; Doyle, B.; Datta, S.; Kavalieros, J.; Zhang, K. Nat. Mater. 2007, $6(11), 810-812$.

[6] Efros, A.; Brus, L. ACS Nano 2021, 15(4), 6192-6210.

[7] Lu, W.; Lieber, C. J. Phys. D: Appl. Phys. 2006, 39(21), R387-R406.

[8] Lu, W.; Xie, P.; Lieber, C. IEEE transactions on Electron Devices 2008, 5(11), $2859-2876$.

[9] Schmidt, V.; Wittemann, J.; Gosele, U. Chem. Rev. 2010, 110, 361-388. 
[10] Barrigón, E.; Heurlin, M.; Bi, Z.; Monemar, B.; Samuelson, L. Chem. Rev. 2019, 119(15), 9170-9220.

[11] Zeng, M.; Xiao, Y.; Liu, J.; Yang, K.; Fu, L. Chem. Rev. 2018, 118(13), 62366296.

[12] Khan, K.; Tareen, A.; Aslam, M.; Wang, R.; Zhang, Y.; Mahmood, A.; Ouyang, Z.; Zhang, H.; Guo, Z. J. Mater. Chem. C 2020, 8(2), 387-440.

[13] Liu, C.; Chen, H.; Wang, S.; Liu, Q.; Jiang, Y.; Zhang, D.; Liu, M.; Zhou, P. Nat. Nanotechnol. 2020, 15, 545-557.

[14] Martín-Palma, R. J.; Lakhtakia, A. Nanotechnology: A Crash Course; SPIE Digital Library, 2010.

[15] Liz-Marzán, L. M.; Giersig, M. Low-Dimensional Systems: Theory, Preparation, and Some Applications; Springer Science \& Business Media, 2003.

[16] Novoselov, K.; Geim, A.; Morozov, S.; Jiang, D.; Zhang, Y.; Dubonos, S.; Grigorieva, I.; Firsov, A. Science 2004, 306(5696), 666-669.

[17] Zhang, K.; Feng, Y.; Wang, F.; Yang, Z.; Wang, J. J. Mater. Chem. C 2017, 5(46), 11992-12022.

[18] Lauhon, L. J.; Gudiksen, M. S.; Wang, D.; Lieber, C. M. Nature 2002, 420, $57-61$. 
[19] Lu, W.; Xiang, J.; Timko, B. P.; Wu, Y.; Lieber, C. M. Proc. Natl. Acad. Sci. U. S. A. 2005, 102, 10046-10051.

[20] Morales, A. M.; Lieber, C. M. Science 1998, 279(5348), 208-211.

[21] Hu, J.; Odom, T. W.; Lieber, C. M. Acc. Chem. Res. 1999, 32(5), 435-445.

[22] Joselevich, E.; Dai, H.; Liu, J.; Hata, K.; Windle, A. Carbon nanotube synthesis and organization; Springer, Berlin, Heidelberg, 2007.

[23] Kamaras, K.; Itkis, M.; Hu, H.; Zhao, B.; Haddon, R. Science 2003, 301(5639), $1501-1501$.

[24] Kou, L.; Ma, Y.; Sun, Z.; Heine, T.; Chen, C. J. Phys. Chem. Lett. 2017, 8(8), 1905-1919.

[25] Qiao, Z.; Tse, W.; Jiang, H.; Yao, Y.; Niu, Q. Phys. Rev. Lett. 2011, 107(25), 256801.

[26] Park, M.; Kim, Y.; Cho, G.; Lee, S. Phys. Rev. Lett. 2019, $123(21), 216803$.

[27] Wickramaratne, D.; Weston, L.; Van de Walle, C. J. Phys. Chem. C 2018, 122(44), 25524-25529.

[28] Blase, X.; Rubio, A.; Louie, S.; Cohen, M. EPL 1994, 28(5), 335.

[29] Cohen, M.; Zettl, A. Phys. Today 2010, 63(11), 34-38. 
[30] Gong, C.; Li, L.; Li, Z.; Ji, H.; Stern, A.; Xia, Y.; Cao, T.; Bao, W.; Wang, C.; Wang, Y.; Qiu, Z. Nature 2017, 546(7657), 265-269.

[31] Wang, K.; Hu, T.; Jia, F.; Zhao, G.; Y., L.; Solovyev, I.; Pyatakov, A.; Zvezdin, A.; Ren, W. Nature 2019, 114(9), 092405.

[32] Geim, A.; Novoselov, K. Nat. Mater. 2007, 6, 183-191.

[33] Seifert, G.; Terrones, H.; Terrones, M.; Frauenheim, T. Solid State Commun. 2000, 115(12), 635-638.

[34] Sofiah, A.; Samykano, M.; Kadirgama, K.; Mohan, R.; Lah, N. Appl. Mater. Today 2018, 11, 320-337.

[35] Cao, Y.; Fatemi, V.; Fang, S.; Watanabe, K.; Taniguchi, T.; Kaxiras, E.; JarilloHerrero, P. Nature 2018, 556(7699), 43-50.

[36] Yokoya, T.; Kiss, T.; Chainani, A.; Shin, S.; Nohara, M.; Takagi, H. Science 2001, $294(5551), 2518-2520$.

[37] Xiang, J.; Lu, W.; Hu, Y.; Wu, Y.; Yan, H.; Lieber, C. M. Nature 2006, 441, 489-493.

[38] Hu, Y.; Kuemmeth, F.; Lieber, C. M.; Marcus, C. M. Nat. Nanotechnol. 2012, 7, 47-50.

[39] Xiang, J.; Vidan, A.; Tinkham, M.; Westervelt, R. M.; Lieber, C. M. Nat. Nanotechnol. 2006, 1, 208-213. 
[40] Liang, G.; Xiang, J.; Kharche, N.; Klimeck, G.; Lieber, C. M.; Lundstrom, M. Nano Lett. 2007, 7, 642-646.

[41] Hu, Y.; Xiang, J.; Liang, G.; Yan, H.; Lieber, C. M. Nano Lett. 2008, 8, 925-930.

[42] Miranti, R.; Shin, D.; Septianto, R.; Ibáñez, M.; Kovalenko, M.; Matsushita, N.; Iwasa, Y.; Bisri, S. ACS Nano 2020, 14(13), 3242-3250.

[43] Yan, H.; Choe, H.; Nam, S.; Hu, Y.; Das, S.; Klemic, J.; Ellenbogen, J.; Lieber, C. Nature 2011, 470(7333), 240-244.

[44] Žutić, I.; Fabian, J.; Sarma, S. D. Rev. Mod. Phys. 2004, 76, 323-410.

[45] Fert, A. Rev. Mod. Phys. 2008, 80, 1517-1530.

[46] Jansen, R. Nat. Mater. 2012, 11, 400-408.

[47] Sanvito, S. Chem. Soc. Rev. 2011, 40, 3336-3355.

[48] Sanvito, S. Nat. Phys. 2010, 6, 562-564.

[49] Radovanovic, P. V.; Barrelet, C. J.; Gradecak, S.; Qian, F.; Lieber, C. M. Nano Lett. 2005, 5, 1407-1411.

[50] Wu, H. W.; Tsai, C. J.; Chen, L. J. Appl. Phys. Lett. 2007, 90, 043121.

[51] Kazakova, O.; Kulkarni, J. S.; Holmes, J. D.; Demokritov, S. O. Phys. Rev. B: Condens. Matter Mater. Phys. 2005, 72, 094415. 
[52] Arantes, J. T.; da Silva, A. J.; Fazzio, A. Phys. Rev. B: Condens. Matter Mater. Phys. 2007, 75, 115113.

[53] Arantes, J. T.; da Silva, A. J.; Fazzio, A.; Antonelli, A. Phys. Rev. B: Condens. Matter Mater. Phys. 2007, 75, 075316.

[54] Van der Meulen, M. I.; Petkov, N.; Morris, M. A.; Kazakova, O.; Han, X.; Wang, K. L.; Jacob, A. P.; Holmes, J. D. Nano Lett. 2009, 9, 50-56.

[55] Giorgi, G.; Cartoixà, X.; Sgamellotti, A.; Rurali, R. Phys. Rev. B: Condens. Matter Mater. Phys. 2008, 78, 115327.

[56] Durgun, E.; Çakır, D.; Akman, N.; Ciraci, S. Phys. Rev. Lett. 2007, 99, 256806.

[57] Aryal, S.; Pati, R. Nanoscale Adv. 2020, 2(5), 1843-1849.

[58] Wadley, P.; Howells, B.; Železný, J.; Andrews, C.; Hills, V.; Campion, R. P.; Novák, V.; Olejník, K.; Maccherozzi, F.; Dhesi, S. S.; Martin, S. Y.; Wagner, T.; Wunderlich, J.; Freimuth, F.; Mokrousov, Y.; Kuneš, J.; Chauhan, J. S.; Grzybowski, M. J.; Rushforth, A. W.; Edmond, K. W.; Gallagher, B. L.; Jungwirth, T. Science 2016, 6273, 597-590.

[59] Baltz, V.; Manchon, A.; Tsoi, M.; Moriyama, T.; Ono, T.; Tserkovnyak, Y. Rev. Mod. Phys. 2018, 90(1), 015005.

[60] Němec, P.; Fiebig, M.; Kampfrath, T.; Kimel, A. V. Nat. Phys. 2018, 14(3), $229-241$. 
[61] Jungwirth, T.; Marti, X.; Wadley, P.; Wunderlich, J. Nat. Nanotechnol. 2016, 11(3), 231-241.

[62] Jungwirth, T.; Sinova, J.; Manchon, A.; Marti, X.; Wunderlich, J.; Felser, C. Nat. Phys. 2018, 14(3), 200-203.

[63] Kimel, A. V.; Kirilyuk, A.; Tsvetkov, A.; Pisarev, R. V.; Rasing, T. Nature 2004, 429(6994), 850-853.

[64] Kampfrath, T.; Sell, A.; Klatt, G.; Pashkin, A.; Mahrlein, S.; Dekorsy, T.; Wolf, M.; Fiebig, M.; Leitenstorfer, A.; Huber, R. Nat. Photonics 2011, 5(1), $31-34$.

[65] Tzschaschel, C. amd Otani, K.; Iida, R.; Shimura, T.; Ueda, H.; Günther, S.; Fiebig, M.; Satoh, T. Phys. Rev. B: Condens. Matter Mater. Phys. 2017, 95(17), 174407.

[66] Olejník, K.; Seifert, T.; Kašpar, Z.; Novák, V.; Wadley, P.; Campion, R. P.; Baumgartner, M.; Gambardella, P.; Němec, P.; Wunderlich, J.; Sinova, J.; Kuzel, P.; Müller, M.; Kampfrath, T.; Jungwirth, T. Sci. Adv. 2018, 4(3), eaar3566.

[67] Lifshitz, E.; Brumer, M.; Kigel, A.; Sashchiuk, A.; Bashouti, M.; Sirota, M.; Galun, E.; Burshtein, Z.; Le Quang, A.; Ledoux-Rak, I.; Zyss, J. J. Phys. Chem. B 2006, 110(50), 25356-25365. 
[68] Hollingsworth, J. Chem. Mater. 2013, 25(8), 1318-1331.

[69] Cirloganu, C.; Padilha, L.; Lin, Q.; Makarov, N.; Velizhanin, K.; Luo, H.; Robel, I.; Pietryga, J.; Klimov, V. Nat. Commun. 2014, 5(1), 1-8.

[70] Wang, J.; Mora-Seró, I.; Pan, Z.; Zhao, K.; Zhang, H.; Feng, Y.; Yang, G.; Zhong, X.; Bisquert, J. J. Am. Chem. Soc. 2013, 35(42), 15913-15922.

[71] Gao, F.; Lu, Q.; Liu, X.; Yan, Y.; Zhao, D. Nano Lett. 2001, 1(12), 743-748.

[72] Fardy, M.; Hochbaum, A. I.; Goldberger, J.; Zhang, M. M.; Yang, P. Adv. Mater. 2007, 19(19), 3047-3051.

[73] Yang, Y.; Kung, S.; Taggart, D.; Xiang, C.; Yang, F.; Brown, M.; Kruse, T.; Hemminger, J.; Penner, R. Nano Lett. 2008, 8(8), 2447-2451.

[74] Patla, I.; Acharya, S.; Zeiri, L.; Israelachvili, J.; Efrima, S.; Golan, Y. Nano Lett. 2007, 7(6), 1459-1462.

[75] Mukherjee, P. K.; Chatterjee, K.; Chakravorty, D. Phys. Rev. B 2006, 73(3), 035414.

[76] Talapin, D.; Murray, C. Science 2005, 310(5745), 86-89.

[77] Kim, D. K.; Lai, Y.; Vemulkar, T. R.; Kagan, C. R. ACS Nano 2011, 5(12), 10074-10083.

[78] Zhang, J.; Gao, J.; Church, C. P.; Miller, E. M.; Luther, J. M.; Klimov, V. I.; Beard, M. C. Nano Lett. 2014, 14(10), 6010-6015. 
[79] Sayevich, V.; Gaponik, N.; Plötner, M.; Kruszynska, M.; Gemming, T.; Dzhagan, V.; Akhavan, S.; Zahn, D.; Demir, H.; Eychmüller, A. Chem. Mater. 2015, $27(12), 4328-4337$.

[80] Oh, S.; Berry, N.; Choi, J.; Gaulding, E.; Paik, T.; Hong, S.; Murray, C.; Kagan, C. ACS Nano 2013, 7(3), 2413-2421.

[81] Szabo, A.; Ostlund, N. S. Modern Quantum Chemistry; Dover Publications, Mineola, New York, 1996.

[82] Levine, I. S. Quantum Chemistry; Prentice Hall,Upper Saddle River, New Jersey 07458, 2000.

[83] Martin, M. R. Electronic Structure; Cambridge University Press, United Kingdom, 2004.

[84] Born, M.; Oppenheimer, R. Phys. Rev. Lett. 1927, 389(20), 457-484.

[85] Hellmann, H. Leipzig: Franz Deuticke 1937, page 285.

[86] Feynman, R. P. Rev. Mod. Phys. 1939, 56(4), 340-343.

[87] Payne, M. C.; Teter, M. P.; Allan, D. C.; Arias, T. A.; Joannopoulos, J. D. Rev. Mod. Phys. 1992, 64(4), 1045-1097.

[88] Hartree, D. R. Cambridge University Press 1928, 24(1), 389-110.

[89] Hartree, D. R. Cambridge University Press 1928, $24(1), 111-132$. 
[90] Hartree, D. R. Cambridge University Press 1928, $24(3), 426-437$.

[91] Blinder, S. M. American journal of physics 1965, 33(6), 69-89.

[92] Slater, J. C. Phys. Rev. 1930, 35(2), 210-211.

[93] Fock, V. A. Z. Phys. 1930, 61(1), 126.

[94] Hartree, D. R.; Hartree, W. Proc. Royal Soc. Lond. A. 1935, 150(869), 9-33.

[95] Roothaan, C. C. J. Rev. Mod. Phys. 1951, 23(2), 69-89.

[96] Slater, J. C. Phys. Rev. 1951, 81(3), 385.

[97] Slater, J. Phys. Rev. 1929, 34(2), 1293-1322.

[98] Parr, R. G.; Yang, W. Density-Functional Theory of Atoms and Molecules; Oxford University Press, New York, 1989.

[99] Hohenberg, P.; W., K. Phys. Rev. 1964, 136(3B), B864-B871.

[100] Levy, M. Proc. Natl. Acad. Sci. USA 1979, 76(12), 6062-6065.

[101] Levy, M. Phys. Rev. A 1982, 26(3), 1200-1208.

[102] Kohn, W.; Sham, L. J. Phys. Rev. 1965, 140(4A), A1133-A1138.

[103] Capelle, K. Braz. J. Phys. 2006, 36(4A), 1318.

[104] Dirac, P. A. M. Proc. Cambridge Philos. Soc. 1930, 26(3), 376-385. 
[105] Perdew, J. P.; Burke, K.; Ernzerhof, M. Phys. Rev. Lett. 1996, $77(18)$, $3865-3868$.

[106] Becke, A. D. J. Chem. Phys. 1992, 98(2), 1372-1377.

[107] Heyd, J.; Scuseria, G. E.; Ernzerhof, M. J. Chem. Phys. 2003, 118(18), 82078215.

[108] Heyd, J.; Scuseria, G. E. J. Chem. Phys. 2004, 120(16), 7274-7280.

[109] Becke, A. D. J. Chem. Phys. 1993, 98(7), 5648-5652.

[110] Stephens, P. J.; Devlin, F. J.; Chabalowski, C. F.; Frisch, M. J. J. Chem. Phys. 1994, 98(45), 11623-11627.

[111] Kittel, C. Introduction to Solid State Physics; John Wiley and Sons, Inc, 2005.

[112] Ashcroft, N. W.; Mermin, N. D. Solid State Physics; Harcourt College Publishers, 1976.

[113] Monkhorst, H. J.; Pack, J. D. Proc. Natl. Acad. Sci. USA 1979, 76(12), 60626065 .

[114] Monkhorst, H. J.; Pack, J. D. Phys. Rev. B 1976, 13(12), 5188-5192.

[115] Vienna Ab initio Simulation Package (VASP). Kresse, G.; Marsman, M.; Furthmüller, J. 2018. 
[116] Hamann, D. R.; Schlüter, M.; Chiang, C. Phys Rev. Lett. 1979, 43(20), 14941497.

[117] Vanderbilt, D. Phys Rev. B 1990, 41(11), 7892-7895.

[118] Kresse, G.; Joubert, D. Phys. Rev. B 1999, 59(3), 1758-1775.

[119] Hobbs, D.; Kresse, G.; Hafner, J. Phys. Rev. B 2000, 62(17), 11556.

[120] Zeller, R. Computational Nanoscience: Do It Yourself 2006, 31, 419-445.

[121] Marsman, M. https://www.vasp.at/vasp-workshop/magnetism.pdf; VASP, 2003.

[122] Blöchl, P. E. Phys. Rev. B 1994, 50(24), 953-979.

[123] Steiner, S.; Khmelevskyi, S.; Marsmann, M.; Kresse, G. Phys. Rev. B 2016, 93, $1-6$.

[124] Datta, S. Electronic Transport in Mesoscopic Systems; Cambridge University Press: Cambridge, UK, 1995.

[125] Di Ventra, M. Electrical Transport in Nanoscale Systems; Cambridge University Press: Cambridge, New York, 2008.

[126] Sahoo, S. An experimental investigation of spin polarized transport in carbon nanotubes; PhD thesis, University of Basel, 2005.

[127] Tsukagoshi, K.; Alphenaar, B. W.; Ago, H. Nature 1999, 401(6753), 572-574. 
[128] Frisch, M. J.; Trucks, G. W.; Schlegel, H. B.; Scuseria, G. E.; Robb, M. A.; Cheeseman, J. R.; Scalmani, G.; Barone, V.; Petersson, G. A.; Nakatsuji, H.; Li, X.; Caricato, M.; Marenich, A. V.; Bloino, J.; Janesko, B. G.; Gomperts, R.; Mennucci, B.; Hratchian, H. P.; Ortiz, J. V.; Izmaylov, A. F.; Sonnenberg, J. L.; Williams-Young, D.; Ding, F.; Lipparini, F.; Egidi, F.; Goings, J.; Peng, B.; Petrone, A.; Henderson, T.; Ranasinghe, D.; Zakrzewski, V. G.; Gao, J.; Rega, N.; Zheng, G. andLiang, W.; Hada, M.; Ehara, M.; Toyota, K.; Fukuda, R.; Hasegawa, J.; Ishida, M.; Nakajima, T.; Honda, Y.; Kitao, O.; Nakai, H.; Vreven, T.; Throssell, K.; Montgomery, J. A., J.; Peralta, J. E.; Ogliaro, F.; Bearpark, M. J.; Heyd, J. J.; Brothers, E. N.; Kudin, K. N.; Staroverov, V. N.; Keith, T. A.; Kobayashi, R.; Normand, J.; Raghavachari, K.; Rendell, A. P.; Burant, J. C.; Iyengar, S. S.; Tomasi, J.; Cossi, M.; Millam, J. M.; Klene, M.; Adamo, C.; Cammi, R.; Ochterski, J. W.; Martin, R. L.; Morokuma, K.; Farkas, O.; Foresman, J. B.; Fox, D. J. Gaussian 16, Revision C.01; Gaussian, Inc., Wallingford CT 2016.

[129] Landauer, R. IBM J. Res. Dev. 1957, 1(3), 223-231.

[130] Landauer, R. J. Phys.: Cond. Mat. 1989, 1(43), 8099.

[131] Pal, P. P. Quantum transport in a single molecular junction; PhD thesis, Michigan Tech. University, 2011.

[132] Pal, P. P.; Pati, R. Phys Rev. B 2010, 82, 045424. 
[133] Mandal, S.; Pati, R. ACS Nano 2012, 6(4), 3580-3588.

[134] Nah, J.; Dillen, D. C.; Varahramyan, K. M.; Banerjee, S. K.; Tutuc, E. Nano Lett. 2012, 12, 108-112.

[135] Zhao, Y.; Smith, J. T.; Appenzeller, J.; Yang, C. Nano Lett. 2011, 11, 14061411.

[136] Fukata, N.; Mitome, M.; Sekiguchi, T.; Bando, Y.; Kirkham, M.; Hong, J.-I.; Wang, Z. L.; Snyder, R. L. ACS Nano 2012, 6, 8887-8895.

[137] Dillen, D. C.; Kim, K.; Liu, E. S.; Tutuc, E. Nat. Nanotechnol. 2014, 9, 116120.

[138] Higginbotham, A. P.; Larsen, T. W.; Yao, J.; Yan, H.; Lieber, C. M.; Marcus, C. M.; Kuemmeth, F. Nano Lett. 2014, 14, 3582-3586.

[139] Conesa-Boj, S.; Li, A.; Koelling, S.; Brauns, M.; Ridderbos, J.; Nguyen, T. T.; Verheijen, M. A.; Koenraad, P. M.; Zwanenburg, F. A.; Bakkers, E. P. A. M. Nano Lett. 2017, 17, 2259-2264.

[140] Hu, Y.; Churchill, H. O.; Reilly, D. J.; Xiang, J.; Lieber, C. M.; Marcus, C. M. Nat. Nanotechnol. 2007, 2, 622-625.

[141] Goldthorpe, I. A.; Marshall, A. F.; McIntyre, P. C. Nano Lett. 2008, 8, 40814086.

[142] Chang, H. K.; Lee, S. C. Appl. Phys. Lett. 2010, 97, 251912. 
[143] Hao, X. J.; Tu, T.; Cao, G.; Zhou, C.; Li, H. O.; Guo, G. C.; Fung, W. Y.; Ji, Z.; Guo, G. P.; Lu, W. Nano Lett. 2010, 10, 2956-2960.

[144] Park, J. S.; Ryu, B.; Moon, C. Y.; Chang, K. J. Nano Lett. 2010, 10, 116-121.

[145] Jaishi, M.; Pati, R. Nanoscale 2017, 9, 13425-13431.

[146] Duan, X.; Li, Y.; Rajan, N. K.; Routenberg, D. A.; Modis, Y.; Reed, M. A. Nat. Nanotechnol. 2012, 7, 401-407.

[147] Kim, S. K.; Day, R. W.; Cahoon, J. F.; Kempa, T. J.; Song, K. D.; Park, H. G.; Lieber, C. M. Nano Lett. 2012, 12, 4971-4976.

[148] Kempa, T. J.; Cahoon, J. F.; Kim, S. K.; Day, R. W.; Bell, D. C.; Park, H. G.; Lieber, C. M. Proc. Natl. Acad. Sci. U. S. A. 2012, 109, 1407-1412.

[149] Wang, Q.; Sun, Q.; Jena, P. Phys. Rev. Lett. 2005, 95, 167202.

[150] Wang, Q.; Sun, Q.; Jena, P.; Kawazoe, Y. Nano Lett. 2005, 5, 1587-1590.

[151] Jamet, M.; Barski, A.; Devillers, T.; Poydenot, V.; Dujardin, R.; BayleGuillemaud, P.; Rothman, J.; Bellet-Amalric, E.; Marty, A.; Cibert, J.; Mattana, R.; Tatarenko, S. Nat. Mater. 2006, 5, 653-659.

[152] Singh, A. K.; Kumar, V.; Kawazoe, Y. J. Mater. Chem. 2004, 14, 555-563.

[153] Stroppa, A.; Picozzi, S.; Continenza, A.; Freeman, A. J. Phys. Rev. B: Condens. Matter Mater. Phys. 2003, 68, 155203. 
[154] da Silva, A. J. R.; Fazzio, A.; Antonelli, A. Phys. Rev. B: Condens. Matter Mater. Phys. 2004, 70, 193205.

[155] Picozzi, S.; Ottaviano, L.; Passacantando, M.; Profeta, G.; Continenza, A.; Priolo, F.; Kim, M.; Freeman, A. J. Appl. Phys. Lett. 2005, 85, 062501.

[156] Zhou, S.; Schmidt, H. Materials 2010, 3, 5054-5082.

[157] Kresse, G.; Furthmüller, J. Phys. Rev. B: Condens. Matter Mater. Phys. 1996, 54, 11169-11186.

[158] Kresse, G.; Furthmüller, J. Comput. Mater. Sci., 1996, 6, 15-50.

[159] Heyd, J.; Scuseria, G. E. J. Chem. Phys. 2004, 121, 1187-1192.

[160] Mandal, S.; Pati, R. Phys. Rev. B: Condens. Matter Mater. Phys. 2011, 84, 115306.

[161] Mandal, S.; Haule, K.; Rabe, K. M.; Vanderbilt, D. npj Comput. Mater. 2019, 5,115 .

[162] Peng, X.; Tang, F.; Logan, P. J. Phys.: Condens. Matter. 2011, 23, 115502.

[163] Hummer, K.; Harl, J.; Kresse, G. Phys. Rev. B: Condens. Matter Mater. Phys. 2009, 80, 115205 .

[164] Dhungana, K. B.; Pati, R. J. Am. Chem. Soc. 2014, 136, 11494-11498.

[165] Pati, R.; Karna, S. P. Chem. Phys. Lett. 2002, 351, 302-310. 
[166] Kriegner, D.; Výborný, K.; Olejník, K.; Reichlová, H.; Novák, V.; Marti, X.; Gazquez, J.; Saidl, V.; Němec, P.; Volobuev, V.; Springholz, G.; Holý, V.; Jungwirth, T. Nat. Commun. 2016, \%, 11623.

[167] Olejník, K.; Schuler, V.; Martí, X.; Novák, V.; Kašpar, Z.; Wadley, P.; Campion, R.; Edmonds, K.; Gallagher, B.; Garcés, J.; Baumgartner, M.; Gambardella, P.; Jungwirth, T. Nat. Commun. 2017, 8, 15434.

[168] Bossini, D.; Dal Conte, S.; Hashimoto, Y.; Secchi, A.; Pisarev, R.; Rasing, T.; Cerullo, G.; Kimel, A. Nat. Commun. 2016, \%, 10645.

[169] Takei, S.; Halperin, B. I.; Yacoby, A.; Tserkovnyak, Y. Phys. Rev. B: Condens. Matter Mater. Phys. 2014, 90(9), 094408.

[170] Seki, S.; Ideue, T.; Kubota, M.; Kozuka, Y.; Takagi, R.; Nakamura, M.; Kaneko, Y.; Kawasaki, M.; Tokura, Y. Phys. Rev. Lett. 2015, 115(26), 266601.

[171] Wang, H.; Du, C.; Hammel, P. C.; Yang, F. Phys. Rev. Lett. 2014, 113(9), 097202.

[172] Gong, S. J.; Gong, C.; Sun, Y. Y.; Tong, W. Y.; Duan, C. G.; Chu, J. H.; Zhang, X. Proc. Natl. Acad. Sci. U. S. A. 2018, 115(34), 8511-8516.

[173] Bodnar, S.; Šmejkal, L.; Turek, I.; Jungwirth, T.; Gomonay, O.; Sinova, J.; Sapozhnik, A.; Elmers, H.; Kläui, M.; Jourdan, M. Nat. Commun. 2018, 9(1), 348. 
[174] Anderson, P. W. Phys. Rev. 1950, 79(2), 350-356.

[175] Kioseoglou, G.; Hanbicki, A. T.; Li, C. H.; Erwin, S. C.; Goswami, R.; Jonker, B. T. Appl. Phys. Lett. 2004, 84(10), 1725-1727.

[176] Wang, R.; Deacon, R.; Yao, J.; Lieber, C.; Ishibashi, K. Semicond. Sci. Technol. 2017, 32(9), 094002.

[177] Duong, N. P.; Satoh, T.; Fiebig, M. Phys. Rev. Lett. 2004, 93(11), 117402.

[178] Kellermann, K.; Zimin, D.; Alchalabi, K.; Gasser, P.; Pikhtin, N. A.; Zogg, H. J. Appl. Phys. 2003, 94(11), 7053-7058.

[179] Lo, W. Proc. SPIE 1984, 0484, 144-152.

[180] Tacke, M. Phil. Trans. R. Soc. of Lon. A 2001, 359(1780), 547-566.

[181] Zogg, H.; Maissen, C.; Masek, J.; Blunier, S.; Lambrecht, A.; Tacke, M. Semicond. Sci. Technol. 1990, 5(3S), S49-S52.

[182] Chaudhuri, T. K. Int. J. Eng. Res. 1992, 16(6), 481-487.

[183] Pei, Y.; Shi, X.; LaLonde, A.; Wang, H.; Chen, L.; Snyder, G. J. Nature 2011, 473(7345), 66-69.

[184] Yamini, S. A.; Wang, H.; Gibbs, Z. M.; Pei, Y.; Dou, S. X.; Snyder, G. J. Phys. Chem. Chem. Phys. 2014, 16(5), 1835-1840.

[185] Mandal, S.; Pati, R. Chem. Phys. Lett. 2009, 479(4-6), 244-247. 
[186] Peters, J.; de Wit, J.; Vanmaekelbergh, D. Chem. Mater. 2019, 31(5), 16721680.

[187] Jiang, Z.; You, G.; Wang, L.; Liu, J.; Hu, W.; Zhang, Y.; Xu, J. J. Appl. Phys. 2014, 116(8), 084303.

[188] Miranti, R.; Septianto, R.; Ibáñez, M.; Kovalenko, M.; Matsushita, N.; Iwasa, Y.; Bisri, S. Appl. Phys. Lett. 2020, 117(17), 173101.

[189] Krishnamurthy, S.; Singh, A.; Hu, Z.; Blake, A.; Kim, Y.; Singh, A.; Dolgopolova, E.; Williams, D.; Piryatinski, A.; Malko, A.; Htoon, H. Acs Nano 2020, $15(1), 575-587$.

[190] Yanover, D.; Vaxenburg, R.; Tilchin, J.; Rubin-Brusilovski, A.; Zaiats, G.; Capek, R.; Sashchiuk, A.; Lifshitz, E. J. Phy. Chem. C 2014, 118(30), 1700117009.

[191] Mokari, T.; Habas, S. E.; Zhang, M.; Yang, P. Angew. Chem. 2008, 120(30), $5687-5690$.

[192] Huang, K.; Li, Z.; Li, Y.; Yu, N.; Gao, X.; Huang, L.; Lim, S. F.; Han, G. Nano Lett. 2021, 21(12), 4903-4910.

[193] Aryal, S.; Paudyal, D.; Pati, R. Nano Lett. 2021, 21(4), 1856-1862. 
[194] Cummins, D.; Martinez, U.; Kappera, R.; Voiry, D.; Martinez-Garcia, A.; Jasinski, J.; Kelly, D.; Chhowalla, M.; Mohite, A.; Sunkara, M.; Gupta, G. J. Phys. Chem. C 2015, 119(40), 22908-22914.

[195] Koenigsmann, C.; Scofield, M.; Liu, H.; Wong, S. J. Phys. Chem. Lett. 2012, 3(22), 3385-3398.

[196] Percival, S.; Zhang, B. J. Phys. Chem. C 2013, 117(27), 13928-13935.

[197] Kresse, G.; Hafner, J. Phys. Rev. B 1994, 40(20), 14251-14269.

[198] DimmocK, J. O.; Wright, G. B. Phys. Rev. 1964, 135(3A), A821-A830.

[199] Lin, P. J.; Kleinman, L. Phys. Rev. 1966, 142(2), 478-489.

[200] Wei, S. H.; Zunger., A. Phys. Rev. B 1997, 55(20), 13605-13610.

[201] Nimtz, G.; Schlicht, B. Narrow-Gap Semiconductors; Springer-Verlag, New York, 1985.

[202] Ghosh, P.; Kahaly, M. U.; Waghmare., U. V. Phys. Rev. B 2007, 7(24), 245437.

[203] Jiang, J. Z.; Gerward, L.; Secco, R.; Frost, D.; Olsen, J. S.; Truckenbrodt, J. J. Appl. Phys. 2000, 87(5), 2658-2660. 


\section{Appendix A}

\section{Copyrights}

The copyright permission from the Royal Society of Chemistry :

S. Aryal and R. Pati, Nanoscale Adv. 2020, 2, 1843-1849 DOI: 10.1039/C9NA00803A

This copyright permission applies materials included in the Chapter 4 of this thesis, as well as some parts of Chapter 1 (Introduction). 


\section{RE: Permission to reuse my full article in thesis}

CONTRACTS-COPYRIGHT (shared) <Contracts-Copyright@rsc.org>

Wed, Sep 8, 2021 at 7:01 AM

To: "sandipa@mtu.edu" <sandipa@mtu.edu>

Thank you for your enquiry.

As the article you are requesting to reproduce material from is published under a CC-BY (https://creativecommons.org/ licenses/by/3.0/) you do not have to formally request permission to reproduce material from it.

Please note that if the material specified below or any part of it appears with credit or acknowledgement to a third party then you must also secure permission from that third party before reproducing that material.

Please ensure that the published article carries a credit to The Royal Society of Chemistry (see http://rsc.li/permissions for details) and that any electronic version of the work includes a hyperlink to the article on the Royal Society of Chemistry website.

Kind regards,

Matthew Bailey

Matthew Bailey

Contracts and Copyright Executive

Royal Society of Chemistry

Thomas Graham House

Science Park, Milton Road

Cambridge, CB4 OWF, UK

www.rsc.org

-.--Original Message-..-.

From: sandipa@mtu,edu <sandipa@mtu,edu>

Sent: 07 September 2021 22:03

To: CONTRACTS-COPYRIGHT (shared) <Contracts-Copyright@irsc.org>

Subject: Permission to reuse my full article in thesis

Name: Sandip Aryal

Message: Dear Professor,

I want to reuse my article (Nanoscale Adv, 2020,2, 1843-1849) in printed/electronic form in my Ph.D. thesis. I would be extremely grateful if a copyright permission letter to granted to me.

Thank you for your help in advance.

With Regards,

Sandip Aryal 


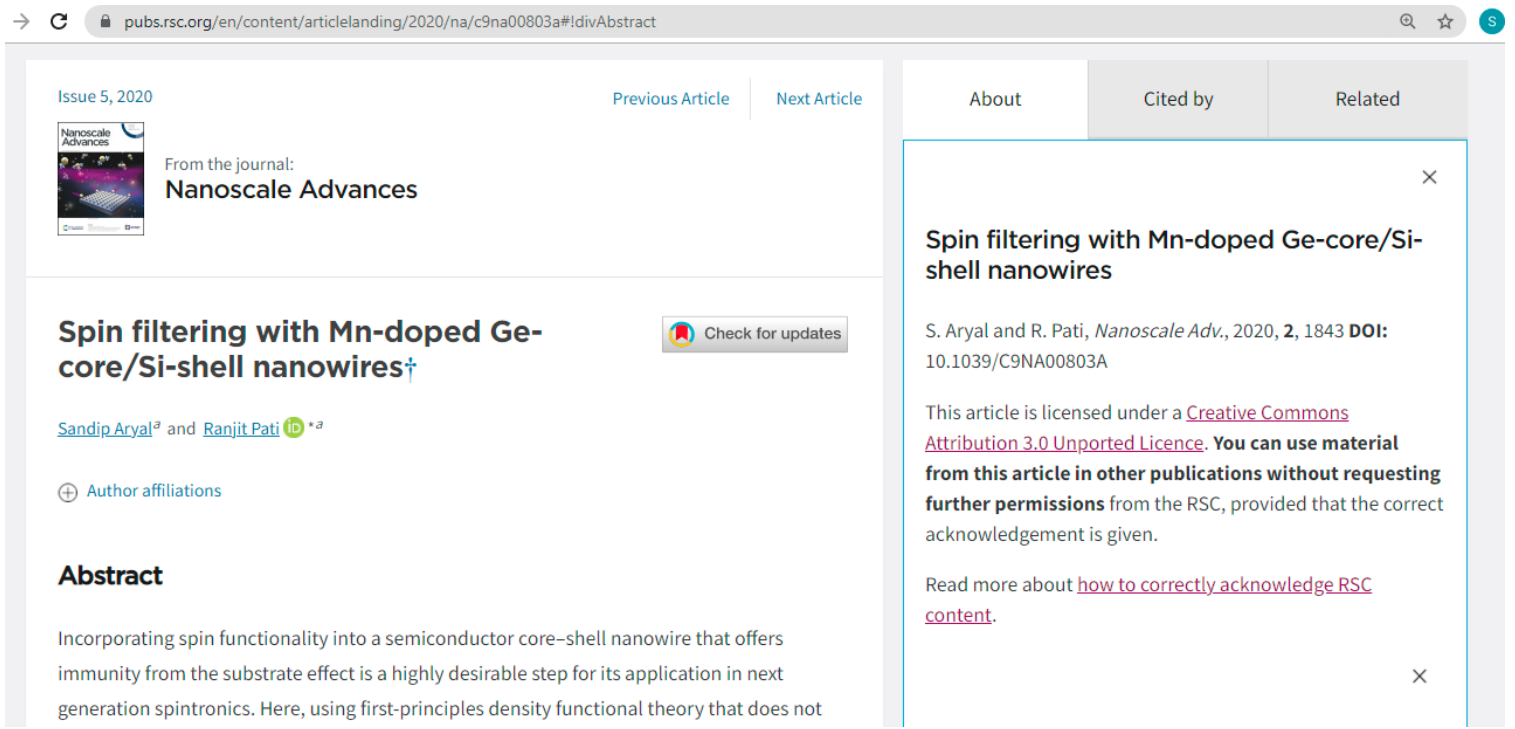





\section{Appendix B}

\section{Copyrights}

The copyright permission from the American chemical society for the article:

S. Aryal, D. Paudyal, and R. Pati, Nano Lett. 2021, 21(4), 1856-1862. DOI:

10.1021/acs.nanolett.0c04971

This copyright permission applies materials included in the Chapter 5 of this thesis, as well as some parts of Chapter 1 (Introduction). 


\section{RightsLink \\ A ? \\ a 2 \\ Home Help $\vee$ Live Chat Sign in Create Account}

\begin{tabular}{|c|c|}
\hline \multirow{6}{*}{ 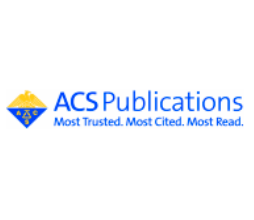 } & Cr-Doped Ge-Core/Si-Shell Nanowire: An Antiferromagnetic Semiconductor \\
\hline & Author: Sandip Aryal, Durga Paudyal, Ranjit Pati \\
\hline & Publication: Nano Letters \\
\hline & Publisher: American Chemical Society \\
\hline & Date: Feb 1, 2021 \\
\hline & Copyright $\odot 2021$, American Chemical Society \\
\hline
\end{tabular}

\section{PERMISSION/LICENSE IS GRANTED FOR YOUR ORDER AT NO CHARGE}

This type of permission/license, instead of the standard Terms and Conditions, is sent to you because no fee is being charged for your order. Please note the following:

- Permission is granted for your request in both print and electronic formats, and translations.

- If figures and/or tables were requested, they may be adapted or used in part.

- Please print this page for your records and send a copy of it to your publisher/graduate school.

- Appropriate credit for the requested material should be given as follows: "Reprinted (adapted) with permission from \{COMPLETE REFERENCE CITATION\}.

Copyright \{YEAR\} American Chemical Society." Insert appropriate information in place of the capitalized words.

- One-time permission is granted only for the use specified in your RightsLink request. No additional uses are granted (such as derivative works or other editions). For any uses, please submit a new request.

If credit is given to another source for the material you requested from RightsLink, permission must be obtained from that source. 


\section{Appendix $\mathrm{C}$}

\section{Copyrights}

The copyright permission from the American chemical society for the article:

S. Aryal and R. Pati, J. Phys. Chem. C 2021. DOI: 10.1021/acs.jpcc.1c06577

This copyright permission applies materials included in the Chapter 6 of this thesis, as well as some parts of Chapter 1 (Introduction). 


\section{RightsLink

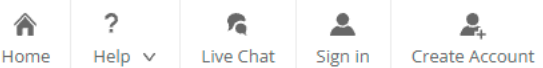

PbTe(core)/PbS(shell) Nanowire: Electronic Structure, Thermodynamic Stability, and Mechanical
and Optical Properties
Author: Sandip Aryal, Ranjit Pati
ACSPublications
Publication: The Journal of Physical Chemistry C
Publisher: American Chemical Society
Date: Oct 1, 2021
Copyright @ 2021, American Chemical Society

\section{PERMISSION/LICENSE IS GRANTED FOR YOUR ORDER AT NO CHARGE}

This type of permission/license, instead of the standard Terms and Conditions, is sent to you because no fee is being charged for your order. Please note the following:

- Permission is granted for your request in both print and electronic formats, and translations.

- If figures and/or tables were requested, they may be adapted or used in part.

- Appropriate credit for the requested material should be given as follows: "Reprinted (adapted) with permission from \{COMPLETE REFERENCE CITATION\}.

Copyright \{YEAR\} American Chemical Society." Insert appropriate information in place of the capitalized words.

- One-time permission is granted only for the use specified in your RightsLink request. No additional uses are granted (such as derivative works or other editions). For any uses, please submit a new request.

If credit is given to another source for the material you requested from RightsLink, permission must be obtained from that source. 